\title{
The weirdest people in the world?
}

\author{
Joseph Henrich \\ Department of Psychology and Department of Economics, University of British \\ Columbia, Vancouver V6T 1Z4, Canada \\ joseph.henrich@gmail.com \\ http://www.psych.ubc.ca/ henrich/home.html
}

Steven J. Heine

Department of Psychology, University of British Columbia, Vancouver

V6T 1Z4, Canada

heine@psych.ubc.ca

Ara Norenzayan

Department of Psychology, University of British Columbia, Vancouver

V6T 1Z4, Canada

ara@psych.ubc.ca

\begin{abstract}
Behavioral scientists routinely publish broad claims about human psychology and behavior in the world's top journals based on samples drawn entirely from Western, Educated, Industrialized, Rich, and Democratic (WEIRD) societies. Researchers - often implicitly - assume that either there is little variation across human populations, or that these "standard subjects" are as representative of the species as any other population. Are these assumptions justified? Here, our review of the comparative database from across the behavioral sciences suggests both that there is substantial variability in experimental results across populations and that WEIRD subjects are particularly unusual compared with the rest of the species - frequent outliers. The domains reviewed include visual perception, fairness, cooperation, spatial reasoning, categorization and inferential induction, moral reasoning, reasoning styles, self-concepts and related motivations, and the heritability of IQ. The findings suggest that members of WEIRD societies, including young children, are among the least representative populations one could find for generalizing about humans. Many of these findings involve domains that are associated with fundamental aspects of psychology, motivation, and behavior - hence, there are no obvious a priori grounds for claiming that a particular behavioral phenomenon is universal based on sampling from a single subpopulation. Overall, these empirical patterns suggests that we need to be less cavalier in addressing questions of human nature on the basis of data drawn from this particularly thin, and rather unusual, slice of humanity. We close by proposing ways to structurally re-organize the behavioral sciences to best tackle these challenges.
\end{abstract}

Keywords: behavioral economics; cross-cultural research; cultural psychology; culture; evolutionary psychology; experiments; external validity; generalizability; human universals; population variability

\section{Introduction}

In the tropical forests of New Guinea, the Etoro believe that for a boy to achieve manhood he must ingest the semen of his elders. This is accomplished through ritualized rites of passage that require young male initiates to fellate a senior member (Herdt 1984/1993; Kelley 1980). In contrast, the nearby Kaluli maintain that male initiation is only properly done by ritually delivering the semen through the initiate's anus, not his mouth. The Etoro revile these Kaluli practices, finding them disgusting. To become a man in these societies, and eventually take a wife, every boy undergoes these initiations. Such boy-inseminating practices, which are enmeshed in rich systems of meaning and imbued with local cultural values, were not uncommon among the traditional societies of Melanesia and Aboriginal Australia (Herdt 1984/1993), as well as in Ancient Greece and Tokugawa Japan.

Such in-depth studies of seemingly "exotic" societies, historically the province of anthropology, are crucial for understanding human behavioral and psychological variation. However, this target article is not about these peoples. It is about a truly unusual group: people from
Western, Educated, Industrialized, Rich, and Democratic (WEIRD $)^{1}$ societies. In particular, it is about the Western, and more specifically American, undergraduates who form the bulk of the database in the experimental branches of psychology, cognitive science, and economics, as well as allied fields (hereafter collectively labeled the "behavioral sciences"). Given that scientific knowledge about human psychology is largely based on findings from this subpopulation, we ask just how representative are these typical subjects in light of the available comparative database. How justified are researchers in assuming a species-level generality for their findings? Here, we review the evidence regarding how WEIRD people compare with other populations.

We pursued this question by constructing an empirical review of studies involving large-scale comparative experimentation on important psychological or behavioral variables. Although such larger-scale studies are highly informative, they are rather rare, especially when compared to the frequency of species-generalizing claims. When such comparative projects were absent, we relied on large assemblies of studies comparing two or three populations, and, when available, on meta-analyses. 
Of course, researchers do not implicitly assume psychological or motivational universality with everything they study. The present review does not address those phenomena assessed by individual difference measures for which the guiding assumption is variability among populations. Phenomena such as personal values, emotional expressiveness, and personality traits are expected a priori to vary across individuals, and by extension, societies. Indeed, the goal of much research on these topics is to identify the ways that people and societies differ from one another. For example, a number of large projects have sought to map out the world on dimensions such as values (Hofstede 2001; Inglehart et al. 1998; Schwartz \& Bilsky 1990), personality traits (e.g., McCrae et al. 2005; Schmitt et al. 2007), and levels of happiness, (e.g., Diener et al. 1995). Similarly, we avoid the vast psychopathology literature, which finds much evidence for both variability and universality in psychological pathologies (Kleinman 1988; Tseng 2001), because this work focuses on individual-level (and unusual) variations in psychological functioning. Instead, we restrict our exploration to

Joseph Henrich holds the Canada Research Chair in Culture, Cognition, and Evolution at the University of British Columbia, where he is appointed Professor in both Economics and Psychology. His theoretical work focuses on how natural selection has shaped human learning and how this in turn influences cultural evolution, and culture-gene coevolution. Methodologically, his research synthesizes experimental and analytical tools drawn from behavioural economics and psychology with in-depth quantitative ethnography, and he has performed long-term fieldwork in the Peruvian Amazon, rural Chile, and in Fiji. Trained in anthropology, Dr. Henrich's work has been published in the top journals in biology, anthropology, and economics. In 2004 he was awarded the Presidential Early Career Award, the highest award bestowed by the United States upon scientists early in their careers. In 2007 he co-authored Why Humans Cooperate. In 2009 the Human Behavior and Evolution Society awarded him their Early Career Award for Distinguished Scientific Contributions.

Ara Norenzayan is an Associate Professor of Psychology at the University of British Columbia, Vancouver. He received his Ph.D. from the University of Michigan in 1999, was a postdoctoral fellow at the Ecole Polytechnique, Paris, and served on the faculty of the University of Illinois, Urbana-Champaign before his appointment at UBC. His most recent work addresses the evolution of religious beliefs and behaviors.

Steven J. Heine is Professor of Psychology and Distinguished University Scholar at the University of British Columbia. Much of his work has focused on how culture shapes people's self-concepts, particularly their motivations for self-esteem. Dr. Heine has received the Early Career Award from the International Society of Self and Identity and the Distinguished Scientist Early Career Award for Social Psychology from the American Psychological Association. He is the author of a textbook entitled Cultural Psychology, published in 2008. those domains which have largely been assumed, at least until recently, to be de facto psychological universals.

Finally, we also do not address societal-level behavioral universals, or claims thereof, related to phenomena such as dancing, fire making, cooking, kinship systems, body adornment, play, trade, and grammar, for two reasons. First, at this surface level alone, such phenomena do not make specific claims about universal underlying psychological or motivational processes. Second, systematic, quantitative, comparative data based on individual-level measures are typically lacking for these domains.

Our examination of the representativeness of WEIRD subjects is necessarily restricted to the rather limited database currently available. We have organized our presentation into a series of telescoping contrasts showing, at each level of contrast, how WEIRD people measure up relative to the available reference populations. Our first contrast compares people from modern industrialized societies with those from small-scale societies. Our second telescoping stage contrasts people from Western societies with those from non-Western industrialized societies. Next, we contrast Americans with people from other Western societies. Finally, we contrast universityeducated Americans with non-university-educated Americans, or university students with non-student adults, depending on the available data. At each level we discuss behavioral and psychological phenomena for which there are available comparative data, and we assess how WEIRD people compare with other samples.

We emphasize that our presentation of telescoping contrasts is only a rhetorical approach guided by the nature of the available data. It should not be taken as capturing any unidimensional continuum, or suggesting any single theoretical explanation for the variation. Throughout this article we take no position regarding the substantive origins of the observed differences between populations. While many of the differences are probably cultural in nature in that they were socially transmitted (Boyd \& Richerson 1985; Nisbett et al. 2001), other differences are likely environmental and represent some form of non-cultural phenotypic plasticity, which may be developmental or facultative, as well as either adaptive or maladaptive (Gangestad et al. 2006; Tooby \& Cosmides 1992). Other population differences could arise from genetic variation, as observed for lactose processing (Beja-Pereira et al. 2003). Regardless of the reasons underlying these population differences, our concern is whether researchers can reasonably generalize from WEIRD samples to humanity at large.

Many radical versions of interpretivism and cultural relativity deny any shared commonalities in human psychologies across populations (e.g., Gergen 1973; see critique and discussion in Slingerland 2008, Ch. 2). To the contrary, we expect humans from all societies to share, and probably share substantially, basic aspects of cognition, motivation, and behavior. As researchers who see great value in applying evolutionary thinking to psychology and behavior, we have little doubt that if a full accounting were taken across all domains among peoples past and present, the number of similarities would indeed be large, as much ethnographic work suggests (e.g., Brown 1991) - ultimately, of course, this is an empirical question. Thus, our thesis is not that humans share few basic psychological properties or processes; rather, we question our current ability to distinguish these reliably developing 
aspects of human psychology from more developmentally, culturally, or environmentally contingent aspects of our psychology given the disproportionate reliance on WEIRD subjects. Our aim here, then, is to inspire efforts to place knowledge of such universal features of psychology on a firmer footing by empirically addressing, rather than a priori dismissing or ignoring, questions of population variability.

\section{Background}

Before commencing with our telescoping contrasts, we first discuss two observations regarding the existing literature: (1) The database in the behavioral sciences is drawn from an extremely narrow slice of human diversity; and (2) behavioral scientists routinely assume, at least implicitly, that their findings from this narrow slice generalize to the species.

\subsection{The behavioral sciences database is narrow}

Who are the people studied in behavioral science research? A recent analysis of the top journals in six subdisciplines of psychology from 2003 to 2007 revealed that $68 \%$ of subjects came from the United States, and a full $96 \%$ of subjects were from Western industrialized countries, specifically those in North America and Europe, as well as Australia and Israel (Arnett 2008). The make-up of these samples appears to largely reflect the country of residence of the authors, as $73 \%$ of first authors were at American universities, and 99\% were at universities in Western countries. This means that 96\% of psychological samples come from countries with only $12 \%$ of the world's population.

Even within the West, however, the typical sampling method for experimental studies is far from representative. In the Journal of Personality and Social Psychology, the premier journal in social psychology - the subdiscipline of psychology that should (arguably) be the most attentive to questions about the subjects' backgrounds $67 \%$ of the American samples (and $80 \%$ of the samples from other countries) were composed solely of undergraduates in psychology courses (Arnett 2008). In other words, a randomly selected American undergraduate is more than 4,000 times more likely to be a research participant than is a randomly selected person from outside of the West. Furthermore, this tendency to rely on undergraduate samples has not decreased over time (Peterson 2001; Wintre et al. 2001). Such studies are therefore sampling from a rather limited subpopulation within each country (see Rozin 2001).

It is possible that the dominance of American authors in psychology publications just reflects that American universities have the resources to attract the best international researchers, and that similar tendencies exist in other fields. However, psychology is a distinct outlier here: $70 \%$ of all psychology citations come from the United States - a larger percentage than any of the other 19 sciences that were compared in one extensive international survey (see May 1997). In chemistry, by contrast, the percentage of citations that come from the United States is only $37 \%$. It seems problematic that the discipline in which there are the strongest theoretical reasons to anticipate population-level variation is precisely the discipline in which the American bias for research is most extreme.

Beyond psychology and cognitive science, the subject pools of experimental economics and decision science are not much more diverse - still largely dominated by Westerners, and specifically Western undergraduates. However, to give credit where it is due, the nascent field of experimental economics has begun taking steps to address the problem of narrow samples. ${ }^{2}$

In sum, the available database does not reflect the full breadth of human diversity. Rather, we have largely been studying the nature of WEIRD people, a certainly narrow and potentially peculiar subpopulation.

\subsection{Researchers often assume their findings are universal}

Sampling from a thin slice of humanity would be less problematic if researchers confined their interpretations to the populations from which they sampled. However, despite their narrow samples, behavioral scientists often are interested in drawing inferences about the human mind and human behavior. This inferential step is rarely challenged or defended - with important exceptions (e.g., Medin \& Atran 2004; Rozin 2001; Triandis 1994; Witkin \& Berry 1975) - despite the lack of any general effort to assess how well results from WEIRD samples generalize to the species. This lack of epistemic vigilance underscores the prevalent, though implicit, assumption that the findings one derives from a particular sample will generalize broadly; one adult human sample is pretty much the same as the next.

Leading scientific journals and university textbooks routinely publish research findings claiming to generalize to "humans" or "people" based on research done entirely with WEIRD undergraduates. In top journals such as Nature and Science, researchers frequently extend their findings from undergraduates to the species - often declaring this generalization in their titles. These contributions typically lack even a cautionary footnote about these inferential extensions.

In psychology, much of this generalization is implicit. A typical article does not claim to be discussing "humans" but will rather simply describe a decision bias, psychological process, set of correlations, and so on, without addressing issues of generalizability, although findings are often linked to "people." Commonly, there is no demographic information about the participants, aside from their age and gender. In recent years there is a trend to qualify some findings with disclaimers such as "at least within Western culture," though there remains a robust tendency to generalize to the species. Arnett (2008) notes that psychologists would surely bristle if journals were renamed to more accurately reflect the nature of their samples (e.g., Journal of Personality and Social Psychology of American Undergraduate Psychology Students). They would bristle, presumably, because they believe that their findings generalize much beyond this sample. Of course, there are important exceptions to this general tendency, as some researchers have assembled a broad database to provide evidence for universality (Buss 
1989; Daly \& Wilson 1988; Ekman 1999b; Elfenbein \& Ambady 2002; Kenrick \& Keefe 1992a; Tracy \& Matsumoto 2008).

When is it safe to generalize from a narrow sample to the species? First, if one had good empirical reasons to believe that little variability existed across diverse populations in a particular domain, it would be reasonable to tentatively infer universal processes from a single subpopulation. Second, one could make an argument that as long as one's samples were drawn from near the center of the human distribution, then it would not be overly problematic to generalize across the distribution more broadly - at least the inferred pattern would be in the vicinity of the central tendency of our species. In the following, with these assumptions in mind, we review the evidence for the representativeness of findings from WEIRD people.

\section{Contrast 1: Industrialized societies versus small-scale societies}

Our theoretical perspective, which is informed by evolutionary thinking, leads us to suspect that many aspects of people's psychological repertoire are universal. However, the current empirical foundations for our suspicions are rather weak because the database of comparative studies that include small-scale societies is scant, despite the obvious importance of such societies in understanding both the evolutionary history of our species and the potential impact of diverse environments on our psychology. Here we first discuss the evidence for differences between populations drawn from industrialized and small-scale societies in some seemingly basic psychological domains, and follow this with research indicating universal patterns across this divide.

\subsection{Visual perception}

Many readers may suspect that tasks involving "low-level" or "basic" cognitive processes such as vision will not vary much across the human spectrum (Fodor 1983). However, in the 1960s an interdisciplinary team of anthropologists and psychologists systematically gathered data on the susceptibility of both children and adults from a wide range of human societies to five "standard illusions" (Segall et al. 1966). Here we highlight the comparative findings on the famed Müller-Lyer illusion, because of this illusion's importance in textbooks, and its prominent role as Fodor's indisputable example of "cognitive impenetrability" in debates about the modularity of cognition (McCauley \& Henrich 2006). Note, however, that population-level variability in illusion susceptibility is not limited to the Müller-Lyer illusion; it was also found for the Sander-Parallelogram and both Horizontal-Vertical illusions.

Segall et al. (1966) manipulated the length of the two lines in the Müller-Lyer illusion (Fig. 1) and estimated the magnitude of the illusion by determining the approximate point at which the two lines were perceived as being of the same length. Figure 2 shows the results from 16 societies, including 14 small-scale societies. The vertical axis gives the "point of subjective equality" (PSE), which measures the extent to which segment "a" must be

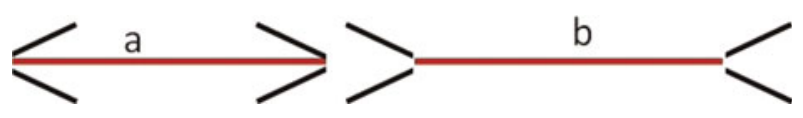

Figure 1. The Müller-Lyer illusion. The lines labeled "a" and "b" are the same length. Many subjects perceive line "b" as longer than line "a".

longer than segment "b" before the two segments are judged equal in length. PSE measures the strength of the illusion.

The results show substantial differences among populations, with American undergraduates anchoring the extreme end of the distribution, followed by the South African-European sample from Johannesburg. On average, the undergraduates required that line "a" be about a fifth longer than line "b" before the two segments were perceived as equal. At the other end, the San foragers of the Kalahari were unaffected by the so-called illusion (it is not an illusion for them). While the San's PSE value cannot be distinguished from zero, the American undergraduates' PSE value is significantly different from all the other societies studied.

As discussed by Segall et al., these findings suggest that visual exposure during ontogeny to factors such as the "carpentered corners" of modern environments may favor certain optical calibrations and visual habits that create and perpetuate this illusion. That is, the visual system ontogenetically adapts to the presence of recurrent features in the local visual environment. Because elements such as carpentered corners are products of particular cultural evolutionary trajectories, and were not part of most environments for most of human history, the MüllerLyer illusion is a kind of culturally evolved by-product (Henrich 2008).

These findings highlight three important considerations. First, this work suggests that even a process as apparently basic as visual perception can show substantial variation across populations. If visual perception can vary, what kind of psychological processes can we be

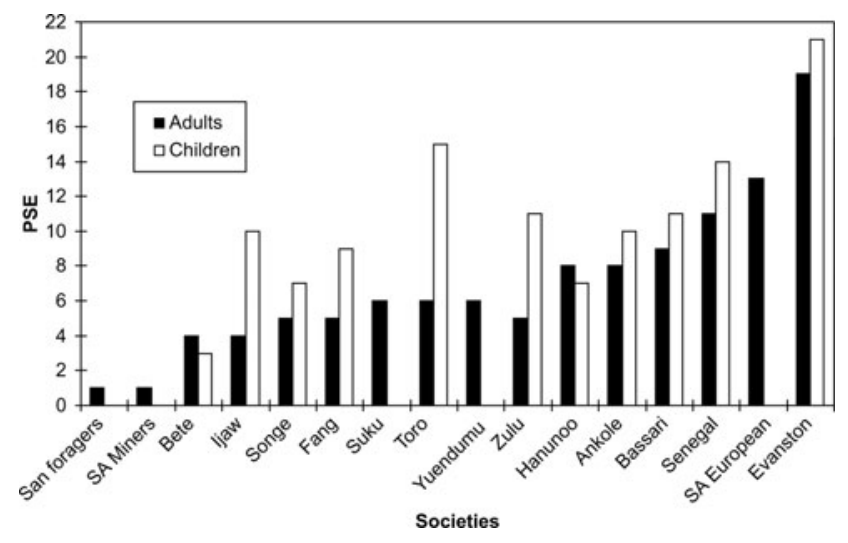

Figure 2. Müller-Lyer results for Segall et al.'s (1966) crosscultural project. PSE (point of subjective equality) is the percentage that segment a must be longer than $b$ before subjects perceived the segments as equal in length. Children were sampled in the 5-to-11 age range. 
sure will not vary? It is not merely that the strength of the illusory effect varies across populations - the effect cannot be detected in two populations. Second, both American undergraduates and children are at the extreme end of the distribution, showing significant differences from all other populations studied; whereas, many of the other populations cannot be distinguished from one another. Since children already show large population-level differences, it is not obvious that developmental work can substitute for research across diverse human populations. Children likely have different developmental trajectories in different societies. Finally, this provides an example of how population-level variation can be useful for illuminating the nature of a psychological process, which would not be as evident in the absence of comparative work.

\subsection{Fairness and cooperation in economic decision-making}

By the mid-1990s, researchers were arguing that a set of robust experimental findings from behavioral economics were evidence for a set of evolved universal motivations (Fehr \& Gächter 1998; Hoffman et al. 1998). Foremost among these experiments, the Ultimatum Game provides a pair of anonymous subjects with a sum of real money for a one-shot interaction. One of the pair - the proposer - can offer a portion of this sum to the second subject, the responder. Responders must decide whether to accept or reject the offer. If a responder accepts, she gets the amount of the offer and the proposer takes the remainder; if she rejects, both players get zero. If subjects are motivated purely by self-interest, responders should always accept any positive offer; knowing this, a selfinterested proposer should offer the smallest non-zero amount. Among subjects from industrialized populations mostly undergraduates from the United States, Europe, and Asia - proposers typically offer an amount between $40 \%$ and $50 \%$ of the total, with a modal offer of $50 \%$ (Camerer 2003). Offers below about $30 \%$ are often rejected.

With this seemingly robust empirical finding in their sights, Nowak et al. (2000) constructed an evolutionary analysis of the Ultimatum Game. When they modeled the Ultimatum Game exactly as played, they did not get results matching the undergraduate findings. However, if they added reputational information, such that players could know what their partners did with others on previous rounds of play, the analysis predicted offers and rejections in the range of typical undergraduate responses. They concluded that the Ultimatum Game reveals humans' species-specific evolved capacity for fair and punishing behavior in situations with substantial reputational influence. But, since the Ultimatum Game is typically played one-shot without reputational information, Nowak et al. argued that people make fair offers and reject unfair offers because their motivations evolved in a world where such interactions were not fitness relevant - thus, we are not evolved to fully incorporate the possibility of non-reputational action in our decision-making, at least in such artificial experimental contexts.

Recent comparative work has dramatically altered this initial picture. Two unified projects (which we call Phase
1 and Phase 2) have deployed the Ultimatum Game and other related experimental tools across thousands of subjects randomly sampled from 23 small-scale human societies, including foragers, horticulturalists, pastoralists, and subsistence farmers, drawn from Africa, Amazonia, Oceania, Siberia, and New Guinea (Henrich et al. 2005; 2006; 2010). Three different experimental measures show that people in industrialized societies consistently occupy the extreme end of the human distribution. Notably, people in some of the smallest-scale societies, where real life is principally face-to-face, behaved in a manner reminiscent of Nowak et al.'s analysis before they added the reputational information. That is, these populations made low offers and did not reject.

To concisely present these diverse empirical findings, we show results only from the Ultimatum and Dictator Games in Phase II. The Dictator Game is the same as the Ultimatum Game except that the second player cannot reject the offer. If subjects are motivated purely by self-interest, they would offer zero in the Dictator Game. Thus, Dictator Game offers yield a measure of "fairness" (equal divisions) among two anonymous people. By contrast, Ultimatum Game offers yield a measure of fairness combined with an assessment of the likelihood of rejection (punishment). Rejections of offers in the Ultimatum Game provide a measure of people's willingness to punish unfairness.

Using aggregate measures, Figure 3 shows that the behavior of the U.S. adult (non-student) sample occupies the extreme end of the distribution in each case. For Dictator Game offers, Figure 3A shows that the U.S. sample has the highest mean offer, followed by the Sanquianga from Colombia, who are renowned for their prosociality (Kraul 2008). The U.S. offers are nearly double that of the Hadza, foragers from Tanzania, and the Tsimane, forager-horticulturalists from the Bolivian Amazon. Figure 3B shows that for Ultimatum Game offers, the United States has the second highest mean offer, behind the Sursurunga from Papua New Guinea. On the punishment side in the Ultimatum Game, Figure 3C shows the income-maximizing offers (IMO) for each population, which is a measure of the population's willingness to punish inequitable offers. IMO is the offer that an income-maximizing proposer would make if he knew the probability of rejection for each of the possible offer amounts. The U.S. sample is tied with the Sursurunga. These two groups have an IMO five times higher than $70 \%$ of the other societies. While none of these measures indicates that people from industrialized societies are entirely unique vis-à-vis other populations, they do show that people from industrialized societies consistently occupy the extreme end of the human distribution.

Analyses of these data show that a population's degree of market integration and its participation in a world religion both independently predict higher offers, and account for much of the variation between populations. Community size positively predicts greater punishment (Henrich et al. 2010). The authors suggest that norms and institutions for exchange in ephemeral interactions culturally coevolved with markets and expanding largerscale sedentary populations. In some cases, at least in their most efficient forms, neither markets nor large populations were feasible before such norms and institutions 
Henrich et al.: The weirdest people in the world?
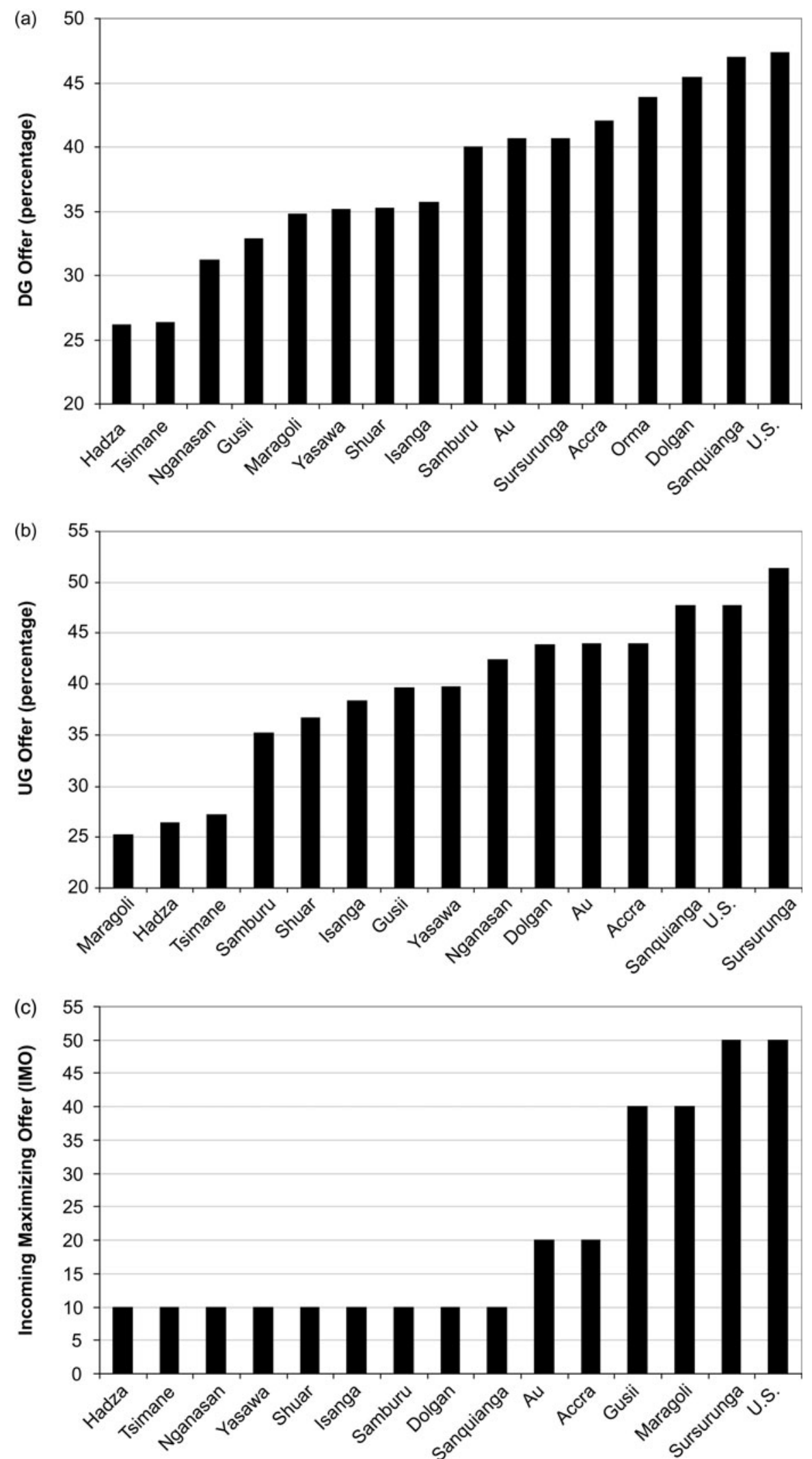

Figure 3. Behavioral measures of fairness and punishment from the Dictator and Ultimatum Games for 15 societies (Phase II). Figures $3 \mathrm{~A}$ and $3 \mathrm{~B}$ show mean offers for each society in the Dictator and Ultimatum Games, respectively. Figure 3C gives the income-maximizing offer (IMO) for each society.

emerged. That is, it may be that what behavioral economists have been measuring among undergraduates in such games is a specific set of social norms, culturally evolved for dealing with money and strangers, that have emerged since the origins of agriculture and the rise of complex societies.

In addition to differences in populations' willingness to reject offers that are too low, the evidence also indicates a 
willingness to reject offers that are too high in about half the societies studied. This tendency to reject so-called hyper-fair offers rises as offers increase from $60 \%$ to $100 \%$ of the stake (Henrich et al. 2006). This phenomenon, which is not observed in typical undergraduate subjects (who essentially never reject offers greater than half), has now emerged among populations in Russia (Bahry \& Wilson 2006) and China (Hennig-Schmidt et al. 2008), as well as (to a lesser degree) among non-student adults in Sweden (Wallace et al. 2007), Germany (Guth et al. 2003), and the Netherlands (Bellemare et al. 2008). Attempts to explain away this phenomenon as a consequence of confusion or misunderstanding, have not found support despite substantial efforts.

Suppose that Nowak and his coauthors were Tsimane, and that the numerous empirical findings they had on hand were all from Tsimane villages. If this were the case, presumably these researchers would have simulated the Ultimatum Game and found that there was no need to add reputation to their model. This unadorned evolutionary solution would have worked fine until they realized that the Tsimane are not representative of humanity. According to the above data, the Tsimane are about as representative of the species as are Americans, but at the opposite end of the spectrum. If the database of the behavioral sciences consisted entirely of Tsimane subjects, researchers would likely be quite concerned about generalizability.

\subsection{Folkbiological reasoning}

Recent work in small-scale societies suggests that some of the central conclusions regarding the development and operation of human folkbiological categorization, reasoning, and induction are limited to urban subpopulations of non-experts in industrialized societies. Although much more work needs to be done, it appears that typical subjects (children of WEIRD parents) develop their folkbiological reasoning in a culturally and experientially impoverished environment, by contrast to those of smallscale societies (and of our evolutionary past), distorting both the species-typical pattern of cognitive development and the patterns of reasoning in WEIRD adults.

Cognitive scientists using (as subjects) children drawn from U.S. urban centers - often those surrounding universities - have constructed an influential, though actively debated, developmental theory in which folkbiological reasoning emerges from folkpsychological reasoning. Before age 7 , urban children reason about biological phenomena by analogy to, and by extension from, humans. Between ages 7 and 10, urban children undergo a conceptual shift to the adult pattern of viewing humans as one animal among many. These conclusions are underpinned by three robust findings from urban children: (1) Inferential projections of properties from humans are stronger than projections from other living kinds; (2) inferences from humans to mammals emerge as stronger than inferences from mammals to humans; and (3) children's inferences violate their own similarity judgments by, for example, providing stronger inference from humans to bugs than from bugs to bees (Carey 1985; 1995).

However, when the folkbiological reasoning of children in rural Native American communities in Wisconsin and
Yukatek Maya communities in Mexico was investigated (Atran et al. 2001; Ross et al. 2003; Waxman \& Medin 2007) none of these three empirical patterns emerged. Among the American urban children, the human category appears to be incorporated into folkbiological induction relatively late compared to these other populations. The results indicate that some background knowledge of the relevant species is crucial for the application and induction across a hierarchical taxonomy (Atran et al. 2001). In rural environments, both exposure to and interest in the natural world is commonplace, unavoidable, and an inevitable part of the enculturation process. This suggests that the anthropocentric patterns seen in U.S. urban children result from insufficient cultural input and a lack of exposure to the natural world. The only real animal that most urban children know much about is Homo sapiens, so it is not surprising that this species dominates their inferential patterns. Since such urban environments are highly "unnatural" from the perspective of human evolutionary history, any conclusions drawn from subjects reared in such informationally impoverished environments must remain rather tentative. Indeed, studying the cognitive development of folkbiology in urban children would seem the equivalent of studying "normal" physical growth in malnourished children.

This deficiency of input likely underpins the fact that the basic-level folkbiological categories for WEIRD adults are life-form categories (e.g., bird, fish, and mammal), and these are also the first categories learned by WEIRD children - for example, if one says "What's that?" (pointing at a maple tree), their common answer is "tree." However, in all small-scale societies studied, the generic species (e.g., maple, crow, trout, and fox) is the basic-level category and the first learned by children (Atran 1993; Berlin 1992).

Impoverished interactions with the natural world may also distort assessments of the typicality of natural kinds in categorization. The standard conclusion from American undergraduate samples has been that goodness of example, or typicality, is driven by similarity relations. A robin is a typical bird because this species shares many of the perceptual features that are commonly found in the category BIRD. In the absence of close familiarity with natural kinds, this is the default strategy of American undergraduates, and psychology has assumed it is the universal pattern. However, in samples which interact with the natural world regularly, such as Itza Maya villagers, typicality is based not on similarity but on knowledge of cultural ideals, reflecting the symbolic or material significance of the species in that culture. For the Itza, the wild turkey is a typical bird because of its rich cultural significance, even though it is in no way most similar to other birds. The same pattern holds for similarity effects in inductive reasoning WEIRD people make strong inferences from computations of similarity, whereas populations with greater familiarity with the natural world, despite their capacity for similarity-based inductions, prefer to make strong inferences from folkbiological knowledge that takes into account ecological context and relationships among species (Atran et al. 2005). In general, research suggests that what people think about can affect how they think (Bang et al. 2007). To the extent that there is population-level variability in the content of folkbiological 
beliefs, such variability affects cognitive processing in this domain as well.

So far we have emphasized differences in folkbiological cognition uncovered by comparative research. This same work has also uncovered reliably developing aspects of human folkbiological cognition that do not vary, such as categorizing plants and animals in a hierarchical taxonomy, or that the generic species level has the strongest inductive potential, despite the fact that this level is not always the basic level across populations, as discussed above. Our goal in emphasizing the differences here is to show (1) how peculiar industrialized (urban, in this case) samples are, given the unprecedented environment they grow up in; and (2) how difficult it is to conclude a priori what aspects will be reliably developing and robust across diverse slices of humanity if research is largely conducted with WEIRD samples.

\subsection{Spatial cognition}

Human societies vary in their linguistic tools for, and cultural practices associated with, representing and communicating (1) directions in physical space, (2) the color spectrum, and (3) integer amounts. There is some evidence that each of these differences in cultural content may influence some aspects of nonlinguistic cognitive processes (D’Andrade 1995; Gordon 2004; Kay 2005; Levinson 2003; Roberson et al. 2000). Here we focus on spatial cognition, for which the evidence is most provocative. As above, it appears that industrialized societies are at the extreme end of the continuum in spatial cognition. Human populations show differences in how they think about spatial orientation and deal with directions, and these differences may be influenced by linguistically based spatial reference systems.

Speakers of English and other Indo-European languages favor the use of an egocentric (relative) system to represent the location of objects - that is, relative to the self (e.g., "the man is on the right side of the flagpole"). In contrast, many if not most languages favor an allocentric frame, which comes in two flavors. Some allocentric languages such as Guugu Yimithirr (an Australian language) and Tzeltal (a Mayan language) favor a geocentric system in which absolute reference is based on cardinal directions ("the man is west of the house"). The other allocentric frame is an object-centered (intrinsic) approach that locates objects in space, relative to some coordinate system anchored to the object ("the man is behind the house"). When languages possess systems for encoding all of these spatial reference frames, they often privilege one at the expense of the others. However, the fact that some languages lack one or more of the reference systems suggests that the accretion of all three systems into most contemporary languages may be a product of longterm cumulative cultural evolution.

In data on spatial reference systems from 20 languages drawn from diverse societies - including foragers, horticulturalists, agriculturalists, and industrialized populations - only three languages relied on egocentric frames as their single preferred system of reference. All three were from industrialized populations: Japanese, English, and Dutch (Majid et al. 2004).

The presence of, or emphasis on, different reference systems may influence nonlinguistic spatial reasoning
(Levinson 2003). In one study, Dutch and Tzeltal speakers were seated at a table and shown an arrow pointing either to the right (north) or the left (south). They were then rotated 180 degrees to a second table where they saw two arrows: one pointing to the left (north) and the other one pointing to the right (south). Participants were asked which arrow on the second table was like the one they saw before. Consistent with the spatial-marking system of their languages, Dutch speakers chose the relative solution, whereas the Tzeltal speakers chose the absolute solution. Several other comparative experiments testing spatial memory and reasoning are consistent with this pattern, although lively debates about interpretation persist (Levinson et al. 2002; Li \& Gleitman 2002).

Extending the above exploration, Haun and colleagues (Haun et al. 2006a; 2006b) examined performance on a spatial reasoning task similar to the one described above, using children and adults from different societies and great apes. In the first step, Dutch-speaking adults and 8 -year-olds (speakers of an egocentric language) showed the typical egocentric bias, whereas Hai//om-speaking adults and 8-year-olds (a Namibian foraging population who speak an allocentric language) showed a typical allocentric bias. In the second step, 4-year-old German-speaking children, gorillas, orangutans, chimpanzees, and bonobos were tested on a simplified version of the same task. All showed a marked preference for allocentric reasoning. These results suggest that children share with other great apes an innate preference for allocentric spatial reasoning, but that this bias can be overridden by input from language and cultural routines.

If one were to work on spatial cognition exclusively with WEIRD subjects (say, using subjects from the United States and Europe), one might conclude that children start off with an allocentric bias but naturally shift to an egocentric bias with maturation. The problem with this conclusion is that it would not apply to many human populations, and it may be the consequence of studying subjects from peculiar cultural environments. The next telescoping contrast highlights some additional evidence suggesting that WEIRD people may even be unusual in their egocentric bias vis-à-vis most other industrialized populations.

\subsection{Other potential differences}

We have discussed several lines of data suggesting not only population-level variation, but that industrialized populations are consistently unusual compared to small-scale societies. There are also numerous studies that have found differences between much smaller numbers of samples (usually two samples). In these studies it is impossible to discern who is unusual, the small-scale society or the WEIRD population. For example, one study found that both samples from two different industrialized populations were risk-averse decision makers when facing monetary gambles involving gains (Henrich \& McElreath 2002), whereas both samples from small-scale societies were risk-prone. Risk-aversion for monetary gains may be a recent, local phenomenon. Similarly, extensive inter-temporal choice experiments using a panel method of data collection indicates that the Tsimane, an Amazonian population of forager-horticulturalists, discount the future 10 times more steeply than do 
WEIRD people (Godoy et al. 2004). In Uganda, a study of individual decision-making among small-scale farmers showed qualitatively different deviations from expected utility maximization than is typically found among undergraduates. For example, rather than the inverse S-shape for probabilities in Prospect Theory, a regular S-shape was found. ${ }^{3}$

\subsection{Similarities between industrialized and small-scale societies}

Some larger-scale comparative projects show universal patterns in human psychology. Here we list some noteworthy examples:

1. Some perceptual illusions: We discussed the MüllerLyer illusion above. However, there are illusions, such as the Perspective Drawing Illusion, for which the industrialized populations are not extreme outliers, and for which perception varies little in the populations studied (Segall et al. 1966).

2. Perceiving color: While the number of basic color terms systematically varies across human languages (Regier et al. 2005), the ability to perceive different colors does emerge in small-scale societies (Rivers 1901a), ${ }^{4}$ although terms and categories do influence color perception at the margins (Kay \& Regier 2006).

3. Emotional expression: In studying facial displays of emotions, Ekman and colleagues have shown much evidence for universality in recognition of the "basic" facial expressions of emotions, although this work has included only a small - yet convincing - sampling of small-scale societies (Ekman 1999a; 1999b). There is also evidence for the universality of pride displays (Tracy \& Matsumoto 2008; Tracy \& Robins 2008). This main effect for emotional recognition across population (58\% of variance) is qualified by a smaller effect for cultural specificity of emotional expressions (9\% of variance: Elfenbein \& Ambady 2002).

4. False belief tasks: Comparative work in China, the United States, Canada, Peru, India, Samoa, and Thailand suggests that the ability to explicitly pass the false belief task emerges in all populations studied (Callaghan et al. 2005; Liu et al. 2008), although the age at which subjects can pass the explicit version of the false belief task varies from 4 to at least 9 (Boesch 2007; Callaghan et al. 2005; Liu et al. 2008), with industrialized populations at the extreme low end.

5. Analog numeracy: There is growing consensus in the literature on numerical thinking that quantity estimation relies on a primitive "analog" number sense that is sensitive to quantity but limited in accuracy. This cognitive ability appears to be independent of counting practices and was shown to operate in similar ways among two Amazonian societies with very limited counting systems (Gordon 2004; Pica et al. 2004), as well as in infants and primates (e.g., Dehaene 1997).

6. Social relationships: Research on the cognitive processes underlying social relationships reveals similar patterns across distinct populations. Fiske (1993) studied people's tendency to confuse one person with another (e.g., intending to phone your son Bob but accidentally calling your son Fred). Chinese, Korean, Bengali, and Vai (Liberia and Sierra Leone) immigrants tended to confuse people in the same category of social relationship.
Interestingly, the social categories in which the most confusion occurred varied across populations.

7. Psychological essentialism: Research from a variety of societies, including Vezo children in Madagascar (Astuti et al. 2004), children from impoverished neighborhoods in Brazil (Sousa et al. 2002), Menominee in Wisconsin (Waxman et al. 2007), and middle-class children and adults in the United States (Gelman 2003), shows evidence of perceiving living organisms as having an underlying and non-trivial nature that makes them what they are. Psychological essentialism also extends to the understanding of social groups, which may be found in Americans (Gelman 2003), rural Ukranians (Kanovsky 2007), Vezo in Madagascar (Astuti 2001), Mapuche farmers in Chile (Henrich \& Henrich, unpublished manuscript), Iraqi Chaldeans and Hmong immigrants in Detroit (Henrich \& Henrich 2007), and Mongolian herdsmen (Gil-White 2001). Notably, this evidence is not well suited to examining differences in the degree of psychological essentialism across populations, though it suggests that inter-population variation may be substantial.

There are also numerous studies involving dyadic comparisons between a single small-scale society and a Western population (or a pattern of Western results) in which cross-population similarities have been found. Examples are numerous but include the development of an understanding of death (Barrett \& Behne 2005), shame (Fessler 2004), ${ }^{5}$ and cheater detection (Sugiyama et al. 2002). Finding evidence for similarities across two such disparate populations is an important step towards providing evidence for universality (Norenzayan \& Heine 2005); however, the case would be considerably stronger if it was found across a larger number of diverse populations. ${ }^{6}$

\subsection{Summary for Contrast 1}

Although there are several domains in which the data from small-scale societies appear similar to that from industrialized societies, comparative projects involving visual illusions, social motivations (fairness), folkbiological cognition, and spatial cognition all show industrialized populations as outliers. Given all this, it seems problematic to generalize from industrialized populations to humans more broadly, in the absence of supportive empirical evidence.

\section{Contrast 2: Western ${ }^{7}$ versus non-Western societies}

For our second contrast, we review evidence comparing Western with non-Western populations. Here we examine four of the most studied domains: social decision making (fairness, cooperation, and punishment), independent versus interdependent self-concepts (and associated motivations), analytic versus holistic reasoning, and moral reasoning. We also briefly return to spatial cognition.

\subsection{Anti-social punishment and cooperation}

In the previous contrast, we reviewed social decisionmaking experiments showing that industrialized populations occupy the extreme end of the behavioral 
distribution vis-à-vis a broad swath of smaller-scale societies. Here we show that even among industrialized populations, Westerners are again clumped at the extreme end of the behavioral distribution. Notably, the behaviors measured in the experiments discussed below are strongly correlated with the strength of formal institutions, norms of civic cooperation, and Gross Domestic Product (GDP) per capita.

In 2002, Fehr and Gächter published their classic paper, "Altruistic Punishment in Humans," in Nature, based on Public Goods Games with and without punishment, conducted with undergraduates at the University of Zurich. The paper demonstrated that adding the possibility of punishment to a cooperative dilemma dramatically altered the outcome, from a gradual slide towards little cooperation (and rampant free-riding), to a steady increase towards stable cooperation. Enough subjects were willing to punish non-cooperators at a cost to themselves to shift the balance from free-riding to cooperation. In stable groups this cooperation-punishment combination dramatically increases long-run gains (Gächter et al. 2008).

To examine the generalizability of these results, which many took to be a feature of our species, Herrmann, Thoni, and Gächter conducted systematic comparable experiments among undergraduates from a diverse swath of industrialized populations (Herrmann et al. 2008). In these Public Goods Games, subjects played with the same four partners for 10 rounds and could contribute during each round to a group project. All contributions to the group project were multiplied by 1.6 and distributed equally among all partners. Players could also pay to punish other players by taking money away from them.

In addition to finding population-level differences in the subjects' initial willingness to cooperate, Gächter's team unearthed in about half of these samples a phenomenon that is not observed beyond a trivial degree among typical undergraduate subjects (see our Fig. 4): Many subjects engaged in anti-social punishment; that is, they paid to reduce the earnings of "overly" cooperative individuals (those who contributed more than the punisher did). The effect of this behavior on levels of cooperation was dramatic, completely compensating for the cooperationinducing effects of punishment in the Zurich experiment. Possibilities for altruistic punishment do not generate high levels of cooperation in these populations. Meanwhile, participants from a number of Western countries, such as the United States, the United Kingdom, and Australia, behaved like the original Zurich students. Thus, it appears that the Zurich sample works well for generalizing to the patterns of other Western samples (as well as the Chinese sample), but such findings cannot be readily extended beyond this.

\subsection{Independent and interdependent self-concepts}

Much psychological research has explored the nature of people's self-concepts. Self-concepts are important, as they organize the information that people have about themselves, direct attention to information that is perceived to be relevant, shape motivations, influence how people appraise situations that influence their emotional experiences, and guide their choices of relationship partners. Markus and Kitayama (1991) posited that self-

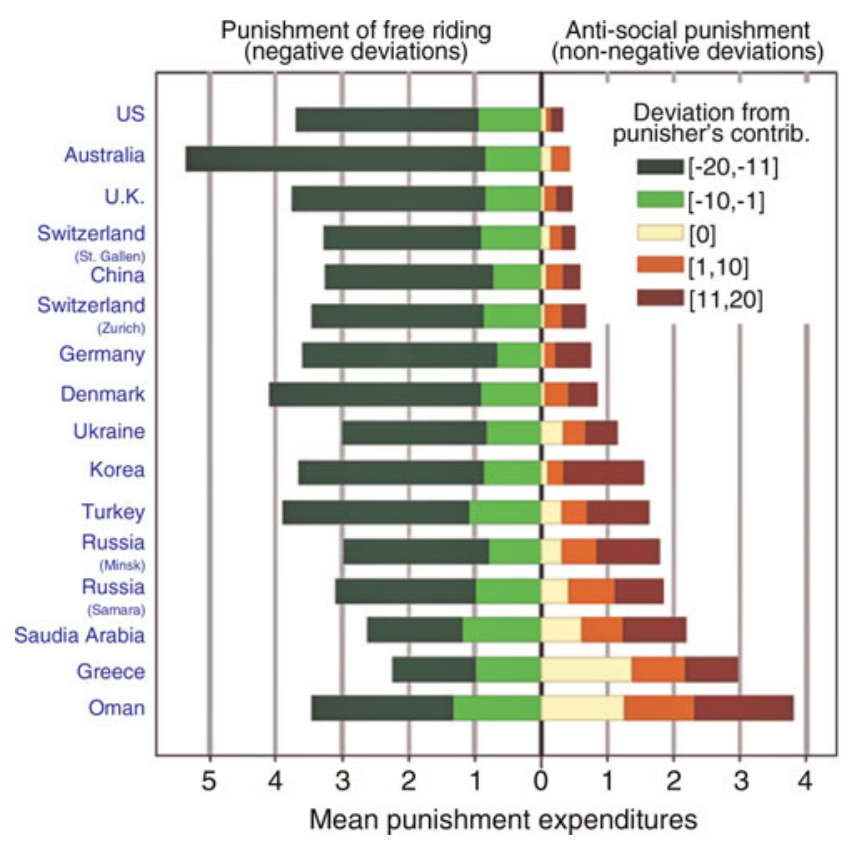

Figure 4. Mean punishment expenditures from each sample for a given deviation from the punisher's contribution to the public good. The deviations of the punished subject's contribution from the punisher's contribution are grouped into five intervals, where $[-20,-11]$ indicates that the punished subjects contributed between 11 and 20 less than the punishing subject; [0] indicates that the punished subject contributed exactly the same amount as the punishing subject; and $[1,10]([11,20])$ indicates that the punished subject contributed between 1 and 10 (11 and 20) more than the punishing subject. Adapted from Herrmann et al. (2008).

concepts can take on a continuum of forms stretching between two poles, termed independent and interdependent self-views, which relate to the individualism-collectivism construct (Triandis 1989; 1994). Do people conceive of themselves primarily as self-contained individuals, understanding themselves as autonomous agents who consist largely of component parts, such as attitudes, personality traits, and abilities? Or do they conceive of themselves as interpersonal beings intertwined with one another in social webs, with incumbent role-based obligations towards others within those networks? The extent to which people perceive themselves in ways similar to these independent or interdependent poles has significant consequences for a variety of emotions, cognitions, and motivations.

Much research has underscored how Westerners have more independent views of self than non-Westerners. For example, research using the Twenty Statements Test (Kuhn \& McPartland 1954) reveals that people from Western populations (e.g., Australians, Americans, Canadians, Swedes) are far more likely to understand their selves in terms of internal psychological characteristics, such as their personality traits and attitudes, and are less likely to understand them in terms of roles and relationships, than are people from non-Western populations, such as Native Americans, Cook Islanders, Maasai and Samburu (both African pastoralists), Malaysians, and East Asians (for a review, see Heine 2008). Studies using other measures (Hofstede 1980; Morling \& Lamoreaux 
2008; Oyserman et al. 2002; Triandis et al. 1990) provide convergent evidence that Westerners tend to have more independent, and less interdependent, self-concepts than those of other populations. These data converge with much ethnographic observation, in particular Geertz's (1975, p. 48) claim that the Western self is "a rather peculiar idea within the context of the world's cultures."

There are numerous psychological patterns associated with self-concepts. For example, people with independent self-concepts are more likely to demonstrate (1) positively biased views of themselves; (2) a heightened valuation of personal choice; and (3) an increased motivation to "stand out" rather than to "fit in." Each of these represents a significant research enterprise, and we discuss them in turn.

4.2.1. Positive self-views. The most widely endorsed assumption regarding the self is that people are motivated to view themselves positively. Roger Brown (1986) famously declared this motivation to maintain high selfesteem an "urge so deeply human, we can hardly imagine its absence" (p. 534). The strength of this motivation has been perhaps most clearly documented by assessing the ways that people go about exaggerating their self-views by engaging in self-serving biases, in which people view themselves more positively than objective benchmarks would justify. For example, in one study, 94\% of American professors rated themselves as better than the average American professor (Cross 1977). However, meta-analyses reveal that these self-serving biases tend to be more pronounced in Western populations than in non-Western ones (Heine \& Hamamura 2007; Mezulis et al. 2004) - for example, Mexicans (Tropp \& Wright 2003), Native Americans (Fryberg \& Markus 2003), Chileans (Heine \& Raineri 2009), and Fijians (Rennie \& Dunne 1994) score much lower on various measures of positive self-views than do Westerners (although there are some exceptions to this general pattern; see Harrington \& Liu 2002). Indeed, in some cultural contexts, most notably East Asian ones, evidence for self-serving biases tends to be null, or in some cases, shows significant reversals, with East Asians demonstrating selfeffacing biases (Heine \& Hamamura 2007). At best, the sharp self-enhancing biases of Westerners are less pronounced in much of the rest of the world, although selfenhancement has long been discussed as if it were a fundamental aspect of human psychology (e.g., Rogers 1951; Tesser 1988).

4.2.2. Personal choice. Psychology has long been fascinated with how people assert agency by making choices (Bandura 1982; Kahneman \& Tversky 2000; Schwartz 2004), and has explored the efforts that people go through to ensure that their actions feel freely chosen and that their choices are sensible. However, there is considerable variation across populations in the extent to which people value choice and in the range of behaviors over which they feel that they are making choices. For example, one study found that European-American children preferred working on a task, worked on it longer, and performed better on it, if they had made some superficial choices regarding the task than if others made the same choices for them. In contrast, Asian-American children were equally motivated by the task if a trusted other made the same choices for them (Iyengar \& Lepper 1999). Another two sets of studies found that Indians were slower at making choices, were less likely to make choices consistent with their personal preferences, and were less likely to view their actions as expressions of choice, than were Americans (Savani et al. 2008; in press). Likewise, the extent to which people feel that they have much choice in their lives varies across populations. Surveys conducted at bank branches in Argentina, Brazil, Mexico, the Philippines, Singapore, Taiwan, and the United States found that Americans were more likely to perceive having more choice at their jobs than were subjects from the other countries (Iyengar \& DeVoe 2003). Another survey administered in more than 40 countries found, in general, that feelings of free choice in one's life were considerably higher in Western nations (e.g., Finland, the United States, and Northern Ireland) than in various non-Western nations (e.g., Turkey, Japan, and Belarus: Inglehart et al. 1998). This research reveals that perceptions of choice are experienced less often, and are a lesser concern, among those from non-Western populations.

4.2.3. Motivations to conform. Many studies have explored whether motivations to conform are similar across populations by employing a standard experimental procedure (Asch 1951; 1952). In these studies, which were initially conducted with Americans, participants first hear a number of confederates making a perceptual judgment that is obviously incorrect, and then participants are given the opportunity to state their own judgment. A majority of American participants were found to go along with the majority's incorrect judgment at least once. This research sparked much interest, apparently because Westerners typically feel that they are acting on their own independent resolve and are not conforming. A meta-analysis of studies performed in 17 societies (Bond \& Smith 1996), including subjects from Oceania, the Middle East, South America, Africa, South America, East Asia, Europe, and the United States, found that motivations for conformity are weaker in Western societies than elsewhere. Other research converges with this conclusion. For example, Kim and Markus (1999) found that Koreans preferred objects that were more common, whereas Americans showed a greater preference for objects that were more unusual.

\subsection{Analytic versus holistic reasoning}

Variation in favored modes of reasoning has been compared across several populations. Most of the research has contrasted Western (American, Canadian, Western European) with East Asian (Chinese, Japanese, Korean) populations with regard to their relative reliance on what is known as "holistic" versus "analytic" reasoning (Nisbett 2003; Peng \& Nisbett 1999). However, growing evidence from other non-Western populations points to a divide between Western nations and most everyone else, including groups as diverse as Arabs, Malaysians, and Russians (see Norenzayan et al. [2007] for a review), as well as subsistence farmers in Africa and South America and sedentary foragers (Norenzayan et al., n.d.; Witkin \& Berry 1975), rather than an East-West divide. 
Holistic thought involves an orientation to the context or field as a whole, including attention to relationships between a focal object and the field, and a preference for explaining and predicting events on the basis of such relationships. Analytic thought involves a detachment of objects from contexts, a tendency to focus on objects' attributes, and a preference for using categorical rules to explain and predict behavior. This distinction between habits of thought rests on a theoretical partition between two reasoning systems. One system is associative, and its computations reflect similarity and contiguity (i.e., whether two stimuli share perceptual resemblances and co-occur in time); the other system relies on abstract, symbolic representational systems, and its computations reflect a rule-based structure (e.g., Neisser 1963; Sloman 1996).

Although both cognitive systems are available in all normal adults, different environments, experiences, and cultural routines may encourage reliance on one system at the expense of the other, giving rise to populationlevel differences in the use of these different cognitive strategies to solve identical problems. There is growing evidence that a key factor influencing the prominence of analytic versus holistic cognition is the different self-construals prevalent across populations. First, independent self-construal primes facilitate analytic processing, whereas interdependent primes facilitate holistic processing (Oyserman \& Lee 2008). Second, geographic regions with greater prevalence of interdependent selfconstruals show more holistic processing, as can be seen in comparisons of Northern and Southern Italians, Hokkaido and mainland Japanese, and Western and Eastern Europeans (Varnum et al. 2008).

Furthermore, the analytic approach is culturally more valued in Western contexts, whereas the holistic approach is more valued in East Asian contexts, leading to normative judgments about cognitive strategies that differ across the respective populations (Buchtel \& Norenzayan 2008). Below we highlight some findings from this research showing that, compared to diverse populations of nonWesterners, Westerners (1) attend more to objects than fields; (2) explain behavior in more decontextualized terms; and (3) rely more on rules over similarity relations to classify objects (for further discussion of the cross-cultural evidence, see Nisbett 2003; Norenzayan et al. 2007).

1. Using evidence derived from the Rod \& Frame Test and Embedded Figures Test, Witkin and Berry (1975) summarize a wide range of evidence from migratory and sedentary foraging populations (Arctic, Australia, and Africa), sedentary agriculturalists, and industrialized Westerners. Only Westerners and migratory foragers consistently emerged at the field-independent end of the spectrum. Recent work among East Asians (Ji et al. 2004) in industrialized societies using the Rod \& Frame Test, the Framed Line Test (Kitayama et al. 2003), and the Embedded Figures Test again shows Westerners at the field-independent end of the spectrum, compared to field-dependent East Asians, Malays, and Russians (Kuhnen et al. 2001). Similarly, Norenzayan et al. (2007) found that Canadians showed less field-dependent processing than did Chinese, who in turn were less field-dependent than were Arabs (also see Zebian \& Denny 2001).

2. East Asians' recall for objects is worse than Americans' if the background has been switched (Masuda \&
Nisbett 2001), indicating that East Asians are attending more to the field. This difference in attention has also been found in saccadic eye-movements as measured with eye-trackers. Americans gaze at focal objects longer than East Asians, who in turn gaze at the background more than Americans (Chua et al. 2005). Furthermore, when performing identical cognitive tasks, East Asians and Westerners show differential brain activation, corresponding to the predicted cultural differences in cognitive processing (Gutchess et al. 2006; Hedden et al. 2008).

3. Several classic studies, initially conducted with Western participants, found that "people" tend to make strong attributions about a person's disposition, even when there are compelling situational constraints (Jones \& Harris 1967; Ross et al. 1977). This tendency to ignore situational information in favor of dispositional information is so commonly observed - among typical subjects - that it was dubbed the "fundamental attribution error" (Ross et al. 1977). However, consistent with much ethnography in non-Western cultures (e.g., Geertz 1975), comparative experimental work demonstrates differences that, while Americans attend to dispositions at the expense of situations (Gilbert \& Malone 1995), East Asians are more likely than Americans to infer that behaviors are strongly controlled by the situation (Miyamoto \& Kitayama 2002; Morris \& Peng 1994; Norenzayan et al. 2002a; Van Boven et al. 1999), particularly when situational information is made salient (Choi \& Nisbett 1998). ${ }^{8}$ Grossmann and Varnum (2010) provides parallel findings with Russians. Likewise, in an investigation of people's lay beliefs about personality across eight populations, Church et al. (2006) found that people from Western populations (i.e., American and Euro-Australian) strongly endorsed the notion that traits remain stable over time and predict behavior over many situations, whereas people from non-Western populations (i.e., Asian-Australian, Chinese-Malaysian, Filipino, Japanese, Mexican, and Malay) more strongly endorsed contextual beliefs about personality, such as ideas suggesting that traits do not describe a person as well as roles or duties do, and that trait-related behavior changes from situation to situation. These patterns are consistent with earlier work on attributions comparing Euro-Americans with Hindu Indians (see Miller 1984; Shweder \& Bourne 1982). Hence, although dispositional inferences can be found outside the West, the fundamental attribution error seems less fundamental elsewhere (Choi et al. 1999).

4. Westerners are also more likely to rely on rules over similarity relations in reasoning and categorization. Chinese subjects were found to be more likely to group together objects which shared a functional (e.g., pencilnotebook) or contextual (e.g., sky-sunshine) relationship, whereas Americans were more likely to group objects together if they belonged to a category defined by a simple rule (e.g., notebook-magazine; Ji et al. 2004). Similarly, work with Russian students (Grossmann, 2010) and Russian small-scale farmers (Luria 1976) showed strong tendencies for participants to group objects according to their practical functions. This appears widespread, as Norenzayan et al. (n.d.) examined classification among the Mapuche and Sangu subsistence farmers in Chile and Tanzania, respectively, and found that their classification resembled the Chinese pattern, although it was exaggerated towards holistic reasoning. 


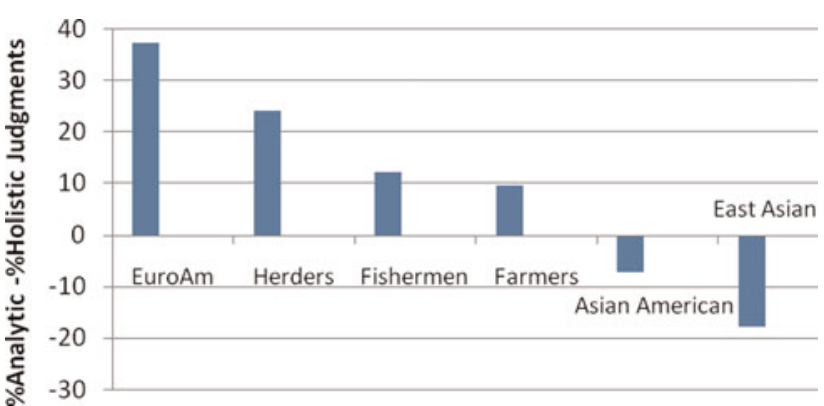

Figure 5. Relative dominance of rule-based versus family resemblance-based judgments of categories for the same cognitive task. European-American, Asian-American, and East Asian university students were tested by Norenzayan et al. (2002b); the herders, fishermen, and farmers of Turkey's Black Sea coast were tested by Uskul et al. (2008). Positive scores indicate a relative bias towards rule-based judgments, whereas negative scores indicate a relative bias towards family resemblance-based judgments. It can be seen that EuropeanAmerican students show the most pronounced bias toward rule-based judgments, and they are outliers in terms of absolute deviation from zero. Adapted from Norenzayan et al. (2002b) and Uskul et al. (2008).

5. In a similar vein, research with East Asians found they were more likely to group objects if the objects shared a strong family resemblance, whereas Americans were more likely to group the same objects if they could be assigned to that group on the basis of a deterministic rule (Norenzayan et al. 2002b). When those results are compared with Uskul et al.'s (2008) findings from herding, fishing, and tea-farming communities on the Black Sea coast in Turkey - the two studies used the same stimuli - it is evident that European-Americans are again at the extreme (see our Figure 5).

In summary, although analytic and holistic cognitive systems are available to all normal adults, a large body of evidence shows that the habitual use of what are considered "basic" cognitive processes, including those involved in attention, perception, categorization, deductive reasoning, and social inference, varies systematically across populations in predictable ways, highlighting the difference between the West and the rest. Several biases and patterns are not merely differences in strength or tendency, but show reversals of Western patterns. We emphasize, however, that Westerners are not unique in their cognitive styles (Uskul et al. 2008; Witkin \& Berry 1975), but they do occupy the extreme end of the distribution.

\subsection{Moral reasoning}

A central concern in the developmental literature has been the way people acquire the cognitive foundations of moral reasoning. The most influential approach to the development of moral reasoning has been Kohlberg's (1971; $1976 ; 1981$ ), in which people's abilities to reason morally are seen to hinge on cognitive abilities that develop over maturation. Kohlberg proposed that people progressed through the same three levels: (1) Children start out at a pre-conventional level, viewing right and wrong as based on internal standards regarding the physical or hedonistic consequences of actions; (2) then they progress to a conventional level, where morality is based on external standards, such as that which maintains the social order of their group; and finally (3) some progress further to a post-conventional level, where they no longer rely on external standards for evaluating right and wrong, but instead do so on the basis of abstract ethical principles regarding justice and individual rights - the moral code inherent in most Western constitutions.

While all of Kohlberg's levels are commonly found in WEIRD populations, much subsequent research has revealed scant evidence for post-conventional moral reasoning in other populations. One meta-analysis carried out with data from 27 countries found consistent evidence for post-conventional moral reasoning in all the Western urbanized samples, yet found no evidence for this type of reasoning in small-scale societies (Snarey 1985). Furthermore, it is not just that formal education is necessary to achieve Kohlberg's post-conventional level. Some highly educated non-Western populations do not show this post-conventional reasoning. At Kuwait University, for example, faculty members scored lower on Kohlberg's schemes than the typical norms for Western adults, and the elder faculty there scored no higher than the younger ones, contrary to Western patterns (AlShehab 2002; Miller et al. 1990).

Research in moral psychology indicates that typical Western subjects rely principally on justice- and harm/ care-based principles in judging morality. However, recent work indicates that non-Western adults and Western religious conservatives rely on a wider range of moral principles than these two dimensions of morality (Baek 2002; Haidt \& Graham 2007; Haidt et al. 1993; e.g., Miller \& Bersoff 1992). Shweder et al. (1997) proposed that in addition to a dominant justice-based morality, which they termed an "ethic of autonomy," there are two other ethics that are commonly found outside the West: an ethic of community, in which morality derives from the fulfillment of interpersonal obligations that are tied to an individual's role within the social order, and an ethic of divinity, in which people are perceived to be bearers of something holy or god-like, and have moral obligations to not act in ways that are degrading to or incommensurate with that holiness. The ethic of divinity requires that people treat their bodies as temples, not as playgrounds, and so personal choices that seem to harm nobody else (e.g., about food, sex, and hygiene) are sometimes moralized (for a further elaboration of moral foundations, see Haidt \& Graham 2007). In sum, the high-socioeconomic status (SES), secular Western populations that have been the primary target of study thus far, appear unusual in a global context, based on their peculiarly narrow reliance, relative to the rest of humanity, on a single foundation for moral reasoning (based on justice, individual rights, and the avoidance of harm to others; cf. Haidt \& Graham 2007).

\subsection{Other potential differences}

There are many other psychological phenomena in which Western samples differ from non-Western ones; however, at present there are insufficient data in these domains derived from diverse populations to assess where Westerners reside in the human spectrum. For example, compared with Westerners, some non-Westerners (1) have 
less dynamic social networks, in which people work to avoid negative interactions among their existing networks rather than seeking new relations (Adams 2005); (2) prefer lower to higher arousal-positive affective states (Tsai 2007); (3) are less egocentric when they try to take the perspective of others (Cohen et al. 2007; Wu \& Keysar 2007); (4) have weaker motivations for consistency (Kanagawa et al. 2001; Suh 2002); (5) are less prone to "social-loafing" (i.e., reducing efforts on group tasks when individual contributions are not being monitored) (Earley 1993); (6) associate fewer benefits with a person's physical attractiveness (Anderson et al. 2008); and (7) have more pronounced motivations to avoid negative outcomes relative to their motivations to approach positive outcomes (Elliot et al. 2001; Lee et al. 2000).

With reference to the spatial reasoning patterns discussed earlier, emerging evidence suggests that a geocentric bias (i.e., a landscape- or earth-fixed spatial coordinate system) may be much more widespread than previously thought indeed, it may be the common pattern outside of the West, even among non-Western speakers of languages which make regular use of egocentric linguistic markers. Comparative research contrasting children and adults in Geneva with samples in Indonesia, Nepal, and rural and urban India have found the typical geocentric reasoning pattern in all of these populations, except for the Geneva samples (Dasen et al. 2006). Although many of these population-level differences are pronounced, more research is needed before we can assess whether the geocentric pattern is common across a broader swath of humanity.

\subsection{Similarities between Western and non-Western societies}

We expect that as more large-scale comparative studies of Western and non-Western populations are conducted, they will reveal substantial similarities in psychological processes. However, given the relative ease of conducting such studies (as compared to working in small-scale societies), there have been few comparative programs that have put universality claims to the test. Here we highlight three examples of larger-scale comparative projects that show broad and important similarities across populations.

1. Mate preferences: First, Buss (1989) compared people from 37 (largely industrialized) populations around the world and found some striking similarities in their mate preferences. In all 37 of the populations, males ranked the physical attractiveness of their mates to be more important than did females; and in 34 of the 37 populations, females ranked the ambition and industriousness of their mates as more important than did males (but for other interpretations, see Eagly \& Wood 1999). ${ }^{9}$ Likewise, Kenrick and Keefe (1992a; 1992b) provide evidence of robust differences in age preferences of mates across populations. Finally, comparative research examining men's preferred waist-to-hip ratios in potential mates finds that men in both industrialized and developing large-scale populations prefer a waist-to-hip ratio of around 0.7 (Singh 2006; Singh \& Luis 1994; Streeter \& McBurney 2003; Swami et al. 2007). ${ }^{10}$

2. Personality structure: Recent efforts have taken personality instruments to university students in 51 different countries (McCrae et al. 2005). In most of these populations, the same five-factor structure emerges that has previously been found with American samples, ${ }^{11}$ indicating the universal structure of the Five Factor Model of personality (also see Allik \& Mccrae 2004; Yik et al. 2002). ${ }^{12}$

3. Punishment of free-riding: While in Hermann et al.'s (2008) study (Fig. 4) both initial cooperation and antisocial punishment varied dramatically, the willingness of players to punish low contributors (free-riders) was not different among populations, once age, sex, and other socio-demographic controls are included.

\subsection{Summary of Contrast 2}

Although robust patterns have emerged among people from industrialized societies, Westerners emerge as unusual - frequent global outliers - on several key dimensions. The experiments reviewed are numerous, arise from different disciplines, use diverse methods, and are often part of systematically comparable data sets created by unified projects. Many of these differences are not merely differences in the magnitude of effects but often show qualitative differences, involving effect reversals or novel phenomena such as allocentric spatial reasoning and antisocial punishment.

\section{Contrast 3: Contemporary Americans versus the rest of the West}

Above we compared WEIRD populations to non-Western populations. However, given the dominance of American research within psychology (see May 1997) and the behavioral sciences, it is important to assess the similarity of American data with that from Westerners more generally. Is it reasonable to generalize from Americans to the rest of the West? Americans are, of course, people too, so they will share many psychological characteristics with other Homo sapiens. At present, we could find no systematic research program to compare Americans with other Westerners, so the evidence presented is assembled from many sources.

\subsection{Individualism and related psychological phenomena}

Americans stand out relative to other Westerners on phenomena that are associated with independent selfconcepts and individualism. A number of analyses, using a diverse range of methods, reveal that Americans are, on average, the most individualistic people in the world (e.g., Hofstede 1980; Lipset 1996; Morling \& Lamoreaux 2008; Oyserman et al. 2002). The observation that the United States is especially individualistic is not new and dates at least as far back as de Toqueville (1835). The unusually individualistic nature of Americans may be caused by, or reflect, an ideology that particularly stresses the importance of freedom and self-sufficiency, as well as various practices in education and childrearing that may help to inculcate this sense of autonomy. American parents, for example, were the only ones in a survey of 100 societies who created a separate room for their baby to sleep (Burton \& Whiting 1961; also see Lewis 1995), reflecting that from the time they are born, Americans are raised in an environment that emphasizes their independence (on the unusual nature of American childrearing, see Lancy 2008; Rogoff 2003) ${ }^{13}$ 
The extreme individualism of Americans is evident on many demographic and political measures. In American Exceptionalism, sociologist Seymour Martin Lipset (1996) documents a long list of the ways that Americans are unique in the Western world. At the time of Lipset's surveys, compared with other Western industrialized societies, Americans were found to be the most patriotic, litigious, philanthropic, and populist (they have the most positions for elections and the most frequent elections, although they have among the lowest voter turnout rates). They were also among the most optimistic, and the least class-conscious. They were the most churchgoing in Protestantism, and the most fundamentalist in Christendom, and were more likely than others from Western industrialized countries to see the world in absolute moral terms. In contrast to other large Western industrialized societies, the United States had the highest crime rate, the longest working hours, the highest divorce rate, the highest rate of volunteerism, the highest percentage of citizens with a post-secondary education, the highest productivity rate, the highest GDP, the highest poverty rate, and the highest income-inequality rate; and Americans were the least supportive of various governmental interventions. The United States is the only industrialized society that never had a viable socialist movement; it was the last country to get a national pension plan, unemployment insurance, and accident insurance; and, at the time of writing, remain the only industrialized nation that does not have a general allowance for families or a national health insurance plan. In sum, there is some reason to suspect that Americans might be different from other Westerners, as de Tocqueville noted.

Given the centrality of self-concept to so many psychological processes, it follows that the unusual emphasis in America on individualism and independence would be reflected in a wide spectrum of self-related phenomena. For example, self-concepts are implicated when people make choices (e.g., Vohs et al. 2008). While Westerners in general tend to value choices more than non-Westerners do (e.g., Iyengar \& DeVoe 2003), Americans value choices more still, and prefer more opportunities, than do Westerners from elsewhere (Savani et al. 2008). For example, in a survey of people from six Western countries, only Americans preferred a choice from 50 different ice cream flavors compared with 10 flavors. Likewise, Americans (and Britons) prefer to have more choices on menus in upscale restaurants than do people from other European countries (Rozin et al. 2006). The array of choices available, and people's motivation to make such choices, is even more extreme in the United States compared to the rest of the West.

Likewise, because cultural differences in analytic and holistic reasoning styles appear to be influenced by whether one views the social world as a collection of discrete individuals or as a set of interconnected relationships (Nisbett 2003), it follows that exceptionally individualistic Americans should be exceptionally analytic as well. One recent study suggests that this might indeed be the case: Americans showed significantly more focused attention in the Framed Line Task than did people from other European countries (Britain and Germany) as well as from Japan (Kitayama et al. 2009). Although more research is needed, Americans may see the world in more analytic terms than the rest of the West.
Terror management theory maintains that because humans possess the conscious awareness that they will someday die, they cope with the associated existential anxiety by making efforts to align themselves with their cultural worldviews (Greenberg et al. 1997). The theory is explicit that the existential problem of death is a human universal, and indeed posits that an awareness of death preceded the evolution of cultural meaning systems in humans (Becker 1973). In support of this argument of universality, the tendency to defend one's cultural worldview following thoughts about death has been found in every one of the more than a dozen diverse populations studied thus far. However, there is also significant crosspopulation diversity in the magnitude of these effects. A recent meta-analysis of all terror management studies reveals that the effect sizes for cultural worldview defense in the face of thoughts of death are significantly more pronounced among American samples $(r=0.37)$ than among other Western $(r=0.30)$ or non-Western samples ( $r=0.26$ : Burke et al. 2010). Curiously, Americans respond more defensively to death thoughts than do those from other countries.

In the previous section, we discussed Herrmann et al.'s (2008) work showing substantial qualitative differences in punishment between Western and non-Western societies. While Western countries all clump at one end of Figure 4, the Americans anchor the extreme end of the West's distribution. Perhaps it is this extreme tendency for Americans to punish free-riders, while not punishing cooperators, that contributes to Americans having the world's highest worker productivity. American society is also anomalous, even relative to other Western societies, in its low relational focus in work settings, which is reflected in practices such as the encouragement of an impersonal work style, direct (rather than indirect) communication, the clear separation of the work domain from the non-work, and discouragement of friendships at work (Sanchez-Burks 2005).

\subsection{Similarities between Americans and other Westerners}

We are unable to locate any research program (other than the ones reviewed in the first two telescoping contrasts) that has demonstrated that American psychological and behavioral patterns are similar to the patterns of other Westerners. We reason that there should be many similarities between the United States and the rest of the West, and we assume that many researchers share our impression. Perhaps this is why we are not able to find studies that have been conducted to explicitly establish these similarities - many researchers likely would not see such studies as worth the effort. In the absence of comparative evidence for a given phenomenon, it might not be unreasonable to assume that the Americans would look similar to the rest of the West. However, the above findings provide a hint that, at least along some key dimensions, Americans are extreme.

\subsection{Summary of Contrast 3}

There are few research programs that have explicitly sought to contrast Americans with other Westerners on psychological or behavioral measures. However, those 
phenomena for which sufficient data are available to make cross-population comparisons reveal that American participants are exceptional even within the unusual population of Westerners - outliers among outliers.

\section{Contrast 4: Typical contemporary American subjects versus other Americans}

The previous contrasts have revealed that WEIRD populations frequently occupy the tail-ends of distributions of psychological and behavioral phenomena. However, it is important to recognize, as a number of researchers have (e.g., Arnett 2008; Medin \& Atran 2004; Sears 1986), that the majority of behavioral research on non-clinical populations within North America is conducted with undergraduates (Peterson 2001; Wintre et al. 2001). Further, within psychology, the subjects are usually psychology majors, or at least taking introductory psychology courses. In the case of child participants, they are often the progeny of high-SES people. Thus, there are numerous social, economic, and demographic dimensions that tentatively suggest that these subjects might be unusual. But, are they?

\subsection{Comparisons among contemporary adult Americans}

Highly educated Americans differ from other Americans in many important respects. In the following subsections, we first highlight findings from social psychology and then from behavioral economics.

6.1.1. Findings from social psychology. For a number of the phenomena reviewed above in which Americans were identified as global outliers, highly educated Americans occupy an even more extreme position than less-educated Americans. Here we itemize eight examples.

1. Although college-educated Americans have been found to rationalize their choices in dozens of postchoice dissonance studies, Snibbe and Markus (2005) found that non-college-educated American adults do not (cf. Sheth 1970).

2. Although Americans are the most individualistic people in the world, American undergraduates score higher on some measures of individualism than do their non-college-educated counterparts, particularly for those aspects associated with self-actualization, uniqueness, and locus of control (Kusserow 1999; Snibbe \& Markus 2005).

3. Conformity motivations were found to be weaker among college-educated Americans than among noncollege-educated Americans (Stephens et al. 2007), who acted in ways more similar to that observed in East Asian samples (cf. Kim \& Markus 1999).

4. Non-college-educated adults are embedded in more tightly structured social networks than are college students (Lamont 2000), which raises the question of whether research on relationship formation, dissolution, and interdependence conducted among students will generalize to the population at large (cf. Adams 2005; Falk et al. 2009).

5. A large study that sampled participants from the general population in southeastern Michigan found that working-class people were more interdependent and more holistic than middle-class people ( $\mathrm{Na}$ et al., in press)
6. The moral reasoning of college-educated Americans occurs almost exclusively within the ethic of autonomy, whereas non-college-educated Americans use the ethics of community and divinity (Haidt et al. 1993; Jensen 1997). Parallel differences exist in moral reasoning between American liberals and conservatives (Haidt \& Graham 2007).

7. American college students respond more favorably toward other groups in society, are more supportive of racial diversity, and are more motivated to mask or explain away negative intergroup attitudes, than are American (non-student) adults (Henry 2009). This difference is more problematic because the percentage of psychological studies of prejudice that exclusively rely on student samples has increased over the last two decades (from $82.7 \%$ to $91.6 \%$ ), and this percentage is accentuated in the higher-impact social psychology journals (Henry 2009).

8. A meta-analysis reveals that college students (the vast majority of whom were American) respond with more cultural worldview defense to death thoughts $(r=0.36)$ than do non-college students $(r=0.25$ : Burke et al. 2010).

More broadly, a second-order meta-analysis $(N>$ $650,000$, Number of studies $>7,000)$ of studies that included either college student samples or non-student adult samples revealed that the two groups differed either directionally or in magnitude for approximately half of the phenomena studied (e.g., attitudes, gender perceptions, social desirability: Peterson 2001). However, no clear pattern regarding the factors that accounted for the differences emerged. Other research has found that American undergraduates have higher degrees of self-monitoring (Reifman et al. 1989), are more susceptible to attitude change (Krosnick \& Alwin 1989), and are more susceptible to social influence (Pasupathi 1999) compared to nonstudent adults.

6.1.2. Findings from behavioral economics. Consistent and non-trivial differences between undergraduates and fully-fledged adults are emerging in behavioral economics as well. When compared with diverse and sometimes representative adult samples, undergraduate subjects consistently set the lower bound for prosociality in experimental measures of trust, fairness, cooperation, and punishment of unfairness or free-riding. For example, in both the Ultimatum and Dictator Games, non-student Americans (both rural and urban participants) make significantly higher offers than do undergraduate subjects (Henrich \& Henrich 2007). The difference is most pronounced in Dictator Games in which samples of non-student American adults from Missouri (urban and rural Missouri did not differ) offered a mean $47 \%$ of the total stake while undergraduate freshmen gave $32 \%$, well within the typical range for undergraduates in this game (Camerer 2003; Ensminger \& Cook, under review; Henrich \& Henrich, under review). These seemingly high offers among non-students in the Dictator Game are similar to those found in other non-student samples in the United States (Carpenter et al. 2005; Henrich \& Henrich 2007). It is the student results that are anomalous. Similarly, more recent research comparing students with both representative and selectively diverse samples of adults using the Trust Game, Ultimatum Game, and Public Goods Game shows that undergraduates ride the lower bound on prosociality measures (Bellemare \& 
Kröger 2007; Bellemare et al. 2008; Carpenter et al. 2008; Fehr \& List 2004). In fact, "being an undergraduate" (or being young and educated) is one of the few demographic variables that seems to matter in explaining within-country variability.

Behavioral economics research also indicates that developmental or acculturative changes to some motivations and preferences are still occurring within the age range of undergraduates (Henrich 2008). For example, Ultimatum Game offers continue to change over the university years, with freshmen making lower offers than seniors (Carter \& Irons 1991). Other work shows that offers do not hit their adult plateau in behavioral games until around age 24 (Carpenter et al. 2005), after which time offers do not change with age until people reach old age. In the Trust Game, measures of trust and trustworthiness increase with age, until they reach a plateau close to age 30 (Sutter \& Kocher 2007a).

Such research may explain why treatment effects also depend on the subject pool used, with students being the most sensitive. For example, Dictator Game treatments involving double-blind setups, such that the experimenter cannot know how much a subject contributes, have dramatically smaller effects on offers among nonstudent adults, and sometimes no effect at all in adult populations outside the United States (Lesorogol \& Ensminger, under review). Similarly, unconscious religious primes increased Dictator Game offers in a Canadian student sample of religious and nonreligious participants alike, but when non-student adults were sampled, no significant effect emerged for the nonreligious adults (Shariff \& Norenzayan 2007).

For several of these economics measures, such as public good contributions (Egas \& Riedl 2008), undergraduate behavior is qualitatively similar to fully-fledged adult behaviors, just less prosocial. However, in at least one area (so far), it appears that a particularly interesting phenomenon is qualitatively absent in undergraduates by comparison with fully-fledged adults from the same populations: As discussed earlier for small-scale societies, researchers using the Ultimatum Game have found systematic, nontrivial tendencies in many populations to reject offers greater than $50 \%$ of the stake, a phenomenon neither previously observed in students nor intuited by researchers. Recent work using representative adult samples has revealed this tendency for "hyper-fair rejections" among non-student adults in Western populations, though it is substantially weaker than in many of the non-Western populations discussed above (Bellemare et al. 2008; Guth et al. 2003; Wallace et al. 2007).

\subsection{Comparisons among subpopulations of American children}

Although studying young children is one important strategy for discerning universals, it does not completely avoid these challenges, as developmental studies are frequently biased toward middle- and upper-class American children. Recent evidence indicates that something as seemingly basic as the differences in spatial reasoning between males and females (Hyde 1981; Mann et al. 1990; Voyer et al. 1995) does not generalize well to poor American children. On two different spatial tasks, repeated four times over two years with 547 second- and third-graders, low-
SES children did not show the sex differences observed in middle- and high-SES children from Chicago (Levine et al. 2005). Such findings, when combined with other research indicating no sex differences on spatial tasks among migratory foragers (Berry 1966), suggest that a proper theory of the origins of sex differences in spatial abilities needs to explain why both poor Chicago children and foragers do not show any sex differences.

Research on IQ using analytical tools from behavioral genetics has long shown that IQ is highly heritable, and not strongly influenced by shared family environment (Bouchard 2004). However, research using 7-year-old twins drawn from a wide range of socioeconomic statuses, shows that contributions of genetic variation and shared environment vary dramatically from low- to high-SES children (Turkheimer et al. 2003). For high-SES children, where environmental variability is negligible, genetic differences account for $70-80 \%$ of the variation, with shared environment contributing less than 10\%. For low-SES children, where there is far more variability in environmental contributions to intelligence, genetic differences account for $0-10 \%$ of the variance, with shared environment contributing about $60 \%$. This raises the specter that much of what we think we have learned from behavioral genetics may be misleading, as the data are disproportionately influenced by WEIRD people and their children (Nisbett 2009).

A similar problem of generalizing from narrow samples exists for genetics research more broadly. Genetic findings obtained with one sample frequently do not replicate in a second sample, to the point that Nature Genetics now requires all empirical papers to include data from two independent samples. There are at least two ways in which geographically limited samples may give rise to spurious genotype-phenotype associations. First, the proportions of various polymorphisms vary across different regions of the world due to different migratory patterns and histories of selection (e.g., Cavalli-Sforza et al. 1994). A genetic association identified in a sample obtained from one region may not replicate in a sample from another region because it involves interactions with other genetic variants that are not equally distributed across regions. Second, the same gene may be expressed differently across populations. For example, Kim et al. (in press) found that a particular serotonin receptor polymorphism (5-HTR1A) was associated with increased attention to focal objects among Americans, but that the same allele was associated with decreased attention to focal objects among Koreans. Researchers would draw different conclusions regarding the function of this polymorphism depending upon the location of their sample. A more complete investigation of heritability and genetic associations demands a comparison of measures across diverse environments and populations.

\subsection{Contemporary Americans compared with previous generations}

Contemporary Americans may also be psychologically unusual compared to their forebears 50 or 100 years ago. Some documented changes among Americans over the past few decades include increasing individualism, as indicated by increasingly solitary lifestyles dominated by individual-centered activities and a decrease in group participation (Putnam 2000), increasingly positive self-esteem 
(Twenge \& Campbell 2001), and a lower need for social approval (Twenge \& Im 2007). These findings suggest that the unusual nature of Americans in these domains, as we reviewed earlier, may be a relatively recent phenomenon. For example, Rozin (2003) found that attitudes towards tradition are more similar between Indian college students and American grandparents than they are between Indian and American college students. Although more research is needed to reach firm conclusions, these initial findings raise doubts as to whether research on contemporary American students (and WEIRD people more generally) is even extendable to American students of previous decades.

The evidence of temporal change is probably best for IQ. Research by Flynn (1987; 2007) shows that IQ scores increased over the last half century by an average of 18 points across all industrialized nations for which there were adequate data. Moreover, this rise was driven primarily by increasing scores on the analytic subtests. This is a striking finding considering recent work showing how unusual Westerners are in their analytic reasoning styles. Given such findings, it seems plausible that Americans of only 50 or 100 years ago were reasoning in ways much more similar to the rest of the non-Western world than Americans of today.

\subsection{Similarities between typical experimental subjects and other Americans}

We expect that typical American subjects are very similar to other Americans in myriad ways. The problem with this expectation, however, is that it is not immediately apparent in which domains they should be similar. We think that there are enough differences between these two groups to raise concerns about speaking incautiously on the thoughts and behaviors of Americans, in general. There have been rather few studies that have explicitly contrasted whether undergraduates or college-educated Americans differ in various psychological measures from those who are not currently students, or who were never college-educated. There are numerous meta-analyses that include data from both college student and nonstudent samples that speak partially to this issue. Although the meta-analyses do not specify the national origin of the participants, we assume that most of the subjects were American. Some of these analyses indicate considerable similarity between student and non-student samples. For example, the aforementioned second-order meta-analysis (Peterson 2001) revealed similarities between students and non-student samples for about half of the phenomena. Similarly, the relation between attribution styles and depression (Sweeney et al. 1986), and the relations among intentions, attitudes, and norms (Farley et al. 1981) do not show any appreciable differences between student and non-student samples. In these instances, there do not appear to be any problems in generalizing from student to non-student samples, which may suggest that college education, and SES more generally, is not related to these phenomena.

\subsection{Summary of Contrast 4}

Numerous findings from multiple disciplines indicate that, in addition to many similarities, there are differences among typical subjects and the rest of the American population in unexpected domains. In some of these domains (e.g., individualism, moral reasoning, worldview defense in response to death thoughts, and perceptions of choice), the data from American undergraduates represent even more dramatic departures from the patterns identified in non-Western samples. Further, contemporary American college students appear further removed along some of these dimensions than did their predecessors a few decades earlier. Typical subjects may be outliers within an outlier population.

\section{General discussion}

As the four contrasts summarized above reveal, WEIRD subjects are unusual in the context of the world in some key ways. In this section, we first discuss the main conclusions and implications of our empirical review. We then address two common challenges to our claim that WEIRD subjects are frequent outliers. Finally, we offer some recommendations for how the behavioral sciences may address these challenges.

\subsection{Summary of our conclusions and implications}

7.1.1. Pronounced population variation is commonplace in the behavioral sciences. There are now enough sources of experimental evidence, using widely differing methods from diverse disciplines, to indicate that there is substantial psychological and behavioral variation among human populations. As we have seen, some of this variability involves differences in the magnitude of effects, motivations, or biases. There is also considerable variability in both whether certain effects or biases exist in some populations (as with antisocial punishment and the Müller-Lyer illusion) and in which direction they go (as with preferences for analytic versus holistic reasoning). The causal origins of such population-level variation may be manifold, including behavioral plasticity in response to different environments, epigenetic effects, divergent trajectories of cultural evolution, and even the differential distribution of genes across groups in response to divergent evolutionary histories. With all these causal possibilities on the table, we think the existence of this population-level variation alone should suffice to energize course corrections in our research directions.

We have also identified many domains in which there are striking similarities across populations. These similarities could indicate reliably developing adaptations (e.g., theory of mind), by-products of innate adaptations (such as some aspects of religious cognition), or independent inventions or diffusions of learned responses that have universal utility (such as counting systems, dance, cooking practices, or techniques for making fire). We have no doubt that there are many more pan-human similarities than we have mentioned (e.g., movement perception, taste for sugar, chunking, habituation, and depth computation); however, thus far there are few databases with individual-level measures sufficient to evaluate the similarities or differences across populations.

Many of the processes identified above that vary dramatically across populations would seem to be "basic" psychological processes. The reviewed findings identified 
variation in aspects of visual perception, memory, attention, fairness motivations, categorization, induction, spatial cognition, self-enhancement, moral reasoning, defensive responses to thoughts about death, and heritability estimates of IQ. These domains are not unique to the social world - they span social as well as nonsocial aspects of the environment, and do not appear to be any less "fundamental" than those domains for which much similarity has been identified. At this point, we know of no strong grounds to make a priori claims to the "fundamentalness" or the likely universality of a given psychological process.

The application of evolutionary theory does not provide grounds for such a priori claims of "fundamental" or "basic" processes, at least in general. Evolutionary theory is a powerful tool for generating and eliminating hypotheses. However, despite its power (or perhaps because of it), it is often overly fecund, as it generates multiple competing hypotheses, with predictions sometimes dependent on unknown or at least debatable aspects of ancestral environments. Hence, adjudicating among alternative evolutionary hypotheses often requires comparative work. Moreover, theoretical work is increasingly recognizing that natural selection has favored ontogenetic adaptations that allow humans, and other species, to adapt non-genetically to local environments (Henrich 2008).

Although we do not yet know of a principled way to predict whether a given psychological process or behavioral pattern will be similar across populations in the absence of comparative empirical research, it would surely be of much value to the field if there were a set of criteria that could be used to anticipate universality (Norenzayan 2006; Norenzayan \& Heine 2005). Here we discuss some possible criteria that might be considered.

First, perhaps there are some domains in which researchers could expect phenomena to be more universal than they are in other domains. We believe that the degree of universality does likely vary across domains, although this has yet to be demonstrated. Many researchers (including us) have the intuition that there are cognitive domains related to attention, memory, and perception in which inter-population variability is likely to be low. Our review of the data, however, does not bolster this intuition. Second, it might be reasonable to assume that some phenomena are more fundamental to the extent that they are measured at a physiological or genetic level, such as genotype-phenotype relations or neural activity. However, recall that the same genes can be expressed differently across populations (e.g., Kim et al., in press), and the same cognitive task may be associated with different neural activations across populations (e.g., Hedden et al. 2008). Third, there may be criteria by which one could confidently make generalizations from one well-studied universal phenomenon to another similar phenomenon; for example, because pride displays are highly similar across populations (e.g., Tracy \& Matsumoto 2008), it might follow that the conceptually related shame display should also be similar across populations as well (Fessler 1999).

Fourth, it would seem that demonstrating a process or effect in other species, such as rats or pigeons, would indicate human universality (and more). Although this may generally be true, several researchers have argued that culture-gene coevolution has dramatically shaped human evolution in a manner uncharacteristic of other species (Richerson \& Boyd 2005). Part of this process may involve the off-loading of previously genetically encoded preferences and abilities into culture (e.g., tastes for spices). Fifth, phenomena which are evident among infants might be reasonably assumed to be more universal than phenomena identified in older children or adults. We suspect this is the case, but it is possible that early biases can be reversed by later ontogeny. Showing parallel findings or effects in both adults and infants from the same population is powerful, and it raises the likelihood of universality; but quite different environments might still shape adult psychologies away from infant patterns (consider the spatial cognition finding with apes, children, and adults). Finally, perhaps particular brain regions are less responsive to experience, such that if a given phenomenon was localized to those regions one could anticipate more universality.

Whatever the relevant principles, it is an important goal to develop theories that predict which elements of our psychological processes are reliably developing across normal human environments and which are locally variable (focusing on the how and why of that variability: Barrett 2006). We note that behavioral scientists have typically been overly confident regarding the universality of what they study, and as this review reveals, our intuitions for what is universal do not have a particularly good track record. We also think this article explains why those intuitions are so poor: Most scientists are WEIRD, or were trained in WEIRD subcultures. Hence, any set of criteria by which universality can be successfully predicted must be grounded in substantial empirical data. We look forward to seeing data that can help to identify criteria to anticipate universality in future research.

7.1.2. WEIRD subjects may often be the worst population from which to make generalizations. The empirical foundation of the behavioral sciences comes principally from experiments with American undergraduates. The patterns we have identified in the available (albeit limited) data indicate that this sub-subpopulation is highly unusual along many important psychological and behavioral dimensions. It is not merely that researchers frequently make generalizations from a narrow subpopulation. The concern is that this particular subpopulation is highly unrepresentative of the species. The fact that WEIRD people are the outliers in so many key domains of the behavioral sciences may render them one of the worst subpopulations one could study for generalizing about Homo sapiens.

To many anthropologically savvy researchers it is not surprising that Americans, and people from modern industrialized societies more generally, appear unusual vis-à-vis the rest of the species. For the vast majority of its evolutionary history, humans have lived in small-scale societies without formal schools, governments, hospitals, police, complex divisions of labor, markets, militaries, formal laws, or mechanized transportation. Every household provisioned much or all of its own food; made its own clothes, tools, and shelters; and - aside from sexual divisions of labor - most everyone had to master the same skills and domains of knowledge. Children typically did not grow up in small, monogamous nuclear families with few kin 
around, nor were they away from their families at school for much of the day.

Rather, through the course of this history, and in some contemporary societies still, children have typically grown up in mixed-age playgroups, where they received little active instruction or exposure to books or TV (Fiske 1998; Lancy 1996; 2008); they learned largely by observation and imitation; received more directives, more physical punishment, and less praise; and were less likely to be engaged in conversation by adults (and there's no "why" phase). By age 10, children in some foraging societies obtain sufficient calories to feed themselves, and routinely kill and butcher animals. Adolescent females in particular take on most of the work-related responsibilities of adult women. People in small-scale societies tend to have less reliable nutrition, greater exposure to hunger, pain, chronic diseases, and lethal dangers, and more frequently experience the death of family members. WEIRD people, from this perspective, grow up in, and adapt to, a rather atypical environment vis-à-vis that of most of human history. It should not be surprising that their psychological world is unusual as well.

7.1.3. Research topics have been limited by the heavy reliance on WEIRD populations. Relying on WEIRD populations may cause researchers to miss important dimensions of variation, and devote undue attention to behavioral tendencies that are unusual in a global context. There are good arguments for choosing topics that are of primary interest to the readers of the literature (i.e., largely WEIRD people); however, if the goal of the research program is to shed light on the human condition, then this narrow, unrepresentative sample may lead to an uneven and incomplete understanding. We suspect that some topics such as self-enhancement, cognitive dissonance, fairness, and analytic reasoning might not have been sufficiently interesting to justify in-depth investigation for most humans at most times throughout history. Alternatively, the behavioral sciences have shown a rather limited interest in such topics as kinship, food, ethnicity (not race), religion, sacred values, polygamy, animal behavior, and rituals (for further critiques on this point, see Rozin 2001; Rozin et al. 2006). Had the behavioral sciences developed elsewhere, important theoretical foci and central lines of research might likely look very different (Medin \& Bang 2008). Moreover, it may be unnecessarily difficult to study psychological phenomena in populations where the phenomena are unusually weak, as is the case for conformity or shame among Americans (see Fessler 2004).

7.1.4. Studying children and primates is crucial, but not a replacement for comparative work. Working with children and nonhuman primates is essential for understanding human psychology. However, it is important to note that despite its great utility and intuitive appeal, such research does not fully obviate these challenges. In the case of primate research, discovering parallel results in great apes and in one human population is an important step, but it doesn't tell us how reliably a particular aspect of psychology develops. As the spatial cognition work indicates, because language and cultural practices can - but need not - influence the cognition humans acquired from their phylogenetic history as apes, establishing the same patterns of cognition in apes and Westerners is insufficient to make any strong claims about universality. Suppose most psychologists were Haillom speakers (instead of Indo-European speakers); they might have studied only Hail\om-speaking children and adults, as well as nonhuman apes, and concluded (incorrectly) that allocentric spatial reasoning was universal. Similarly, imagine if Tsimane economists compared Ultimatum Game results for Tsimane adults to those for chimpanzees (Gurven 2004; Henrich \& Smith 2001; Jensen et al. 2007). These researchers would have found the same results for both species, and concluded that standard game theoretic models (assuming pure self-interest) and evolutionary analyses (Nowak et al. 2000) were fairly accurate predictors in Ultimatum Game behavior for both chimpanzees and humans - a very tidy finding. In both of these cases, the conclusions would be opposite to those drawn from studies with WEIRD populations. ${ }^{14}$

Studying children is crucial for developing universal theories. However, evidence suggests that psychological differences among populations can emerge relatively early in children (as with folkbiological reasoning), and sometimes differences are even larger in children than in adults, as with the Müller-Lyer illusion. Moreover, developmental patterns may be different in different populations, as with sex differences in spatial cognition between low-income versus middle- and high-income subpopulations in the United States, or with performance in the false belief task. This suggests a need for converging lines of research. The most compelling conclusions regarding universality would derive from comparative work among diverse human populations done with both adults and children, including infants if possible. Human work can then be properly compared with work among nonhuman species (including but not limited to primates), based on a combination of field and laboratory work.

\subsubsection{Understanding human diversity is crucial for constructing evolutionary theories of human behavior. Evolution has equipped humans with ontogen-} etic programs, including cultural learning, that help us adapt our bodies and brains to the local physical and social environment. Over the course of human history, convergent forms of cultural evolution have effectively altered (1) our physical environments with tools, technology, and knowledge; (2) our cognitive environments with counting systems, color terms, written symbols, novel grammatical structures, categories, and heuristics; and (3) our social environments with norms, institutions, laws, and punishments. Broad patterns of psychology may be - in part - a product of our genetic program's common response to culturally constructed environments that have emerged and converged over thousands of years. This means that the odd results from small-scale societies, instead of being dismissed as unusual exceptions, ought to be considered as crucial data points that help us understand the ontogenetic processes that build our psychologies in locally adaptive and context-specific ways.

Based on this and the previous point, it seems clear that comparative developmental studies involving diverse human societies combined with parallel studies of nonhuman primates (and other relevant species) provide an approach to understanding human psychology and behavior that can allow us to go well beyond merely establishing 
universality or variability. Such a systematic, multipronged approach can allow us to test a richer array of hypotheses about the processes by which both the reliable universal patterns and the diversity of psychological and behavioral variation emerge.

7.1.6. Exclusive use of WEIRD samples is justified when seeking existential proofs ${ }^{15}$. Our argument should not be construed to suggest that the exclusive use of WEIRD samples should always be avoided. There are cases where the exclusive use of these samples would be legitimate to the extent that generalizability is not a relevant goal of the research, at least initially (Mook 1983). Research programs that are seeking existential proofs for psychological or behavioral phenomena, such as in the case of altruistic punishment discussed earlier (e.g., Fehr \& Gächter 2002), could certainly start with WEIRD samples. That is, if the question is whether a certain phenomenon can be found in humans at all, reliance on any slice of humanity would be a legitimate sampling strategy. For another example, Tversky, Kahneman, and their colleagues sought to demonstrate the existence of systematic biases in decision-making that violate the basic principles of rationality (Gilovich et al. 2002). Most of their work was done with WEIRD samples. Counterexamples to standard rationality predictions could come from any sample in the world. ${ }^{16}$ Furthermore, existential proof for a psychological phenomenon in WEIRD samples can be especially compelling when such a finding is theoretically unexpected. For example, Rozin and Nemeroff (1990) found (surprisingly, to many) that even elite U.S. university students show some magical thinking. Nevertheless, even in such cases, learning about the extent to which population variability affects such phenomena is a necessary subsequent phase of the enterprise, since any theory of human behavior ultimately has to account for such variability (if it exists).

\subsection{Concerns with our argument}

We have encountered two quite different sets of concerns about our argument. Those with the first set of concerns, elaborated below, worry that our findings are exaggerated because (a) we may have cherry-picked only the most extreme cases that fit our argument, and have thus exaggerated the degree to which WEIRD people are outliers, and/or (b) the observed variation across populations may be due to various methodological artifacts that arise from translating experiments across contexts. The second set of concerns is quite the opposite: Some researchers dismissively claim that we are making an obvious point which everyone already recognizes. Perhaps the most productive thing we offer is for these two groups of readers to confront each other.

We preface our response to the first set of concerns with an admonition: Of course, many patterns and processes of human behavior and psychology will be generally shared across the species. We recognize that human thought and behavior is importantly tethered to our common biology and our common experiences. Given this, the real challenge is to design a research program that can explain the manifest patterns of similarity and variation by clarifying the underlying evolutionary and developmental processes.
We offer three general responses to the concern that our review presents a biased picture. To begin, we constructed our empirical review by targeting studies involving important psychological or behavioral concepts which were, or still are, considered to be universal, and which have been tested across diverse populations. We also listed and discussed major comparative studies that have identified important cross-population similarities. Since we have surely overlooked relevant material, we invite commentators to add to our efforts in identifying phenomena which have been widely tested across diverse subpopulations.

Second, we acknowledge that because proper comparative data are lacking for most studied phenomena, we cannot accurately evaluate the full extent of how unusual WEIRD people are. This is, however, precisely the point. We hope research teams will be inspired to span the globe and prove our claims of non-representativeness wrong. The problem is that we simply do not know how well many key phenomena generalize beyond the extant database of WEIRD people. The evidence we present aims only to challenge (provoke?) those who assume that undergraduates are sufficient to make claims about human psychology and behavior.

Third, to address the concern that the observed population-level differences originate from the methodological challenges of working across diverse contexts, we emphasize that the evidence in our article derives from diverse disciplines, theoretical approaches, and methodological techniques. They include experiments involving (1) incentivized economic decisions; (2) perceptual judgments; (3) deceptive experimental practices that prevented subjects from knowing what was being measured; and (4) children, who are less likely than adults to have motivations to shape their responses in ways that they perceive as desirable (or undesirable) to the experimenter. The findings, often published in the best journals of their respective fields, hinged on the researchers making a compelling case that their methodology was comparably meaningful across the populations being studied.

Furthermore, the same methods that have yielded population differences in one domain have demonstrated similarities in other domains (Atran 2005; Haun et al. 2006b; Henrich et al. 2006; Herrmann et al. 2008; Medin \& Atran 2004; Segall et al. 1966). If one wants to highlight the demonstrated similarities, one cannot then ignore the demonstrated differences which relied on the same or similar methodologies.

Note also that few of the findings that we reviewed involve comparing means across subjective self-report measures, for which there are well-known challenges in making cross-population comparisons (Chen et al. 1995; Hamamura et al. 2008; Heine et al. 2002; Norenzayan et al. 2002b; Peng et al. 1997). Therefore, while methodological challenges may certainly be an issue in some specific cases, we think it strains credulity to suggest that such issues invalidate the thrust of our argument, and thus eliminate concerns about the non-representativeness of typical subjects.

\subsection{Our recommendations}

Our experience is that many researchers who work exclusively with WEIRD subjects would like to establish the 
broad generalizability of their findings. Even if they strongly suspect that their findings will generalize across the species, most agree that it would be better to have comparative data across diverse populations. The problem, then, is not exclusively a scientific or epistemological disagreement, but one of institutionalized incentives as well. Hence, addressing this issue will require adjusting the existing incentive structures for researchers. The central focus of these adjustments should be that in presenting our research designs to granting agencies, or our empirical findings in journals, we must explicitly address questions of generalizability and representativeness. With this in mind, we offer the following recommendations.

Journal editors and reviewers should press authors to both explicitly discuss and defend the generalizability of their findings. Claims and confidence regarding generalizability must scale with the strength of the empirical defense. If a result is novel, being explicitly uncertain about generalizability should be fine, but one should not imply universality without an empirically grounded argument.

This does not imply that all experimentalists need to shift to performing comparative work across diverse subject pools! As comparative evidence accumulates in different domains, researchers will be able to assess the growing body of comparative research and thus be able to calibrate their confidence in the generalizability of their findings. The widespread practice of subtly implying universality by using statements such as "people's reasoning is biased..." should be avoided. "Which people?" should be a primary question asked by reviewers. We think this practice alone will energize more comparative work (Rozin 2009).

The experience of evolutionarily-oriented researchers attests to the power of such incentives. More than other researchers in the social sciences, evolutionary researchers have led the way in performing systematic comparative work, drawing data from diverse societies. This is not because they are interested in variation per se (though some are), but because they are compelled, through some combination of their scientific drive and the enthusiasm of their critics, to test their hypotheses in diverse populations (e.g., Billing \& Sherman 1998; Buss 1989; Daly \& Wilson 1988; Fessler et al. 2005; Gangestad et al. 2006; Henrich et al. 2005; Kenrick \& Keefe 1992a; 1992b; Low 2000; Medin \& Atran 2004; Schaller \& Murray 2008; Schmitt 2005; Sugiyama et al. 2002; Tracy \& Robins 2008).

Meta-analyses are often compromised because many studies provide little background information about the subjects. Journal editors should require explicit and detailed information on subject-pool composition (see Rozin 2001). Some granting agencies already require this. Comparative efforts would also be greatly facilitated if researchers would make their data readily available to any who asked; or, better yet, data files should be made available online. Sadly, a recent investigation found that only $27 \%$ of authors in psychology journals shared their data when an explicit request was made to them to do so in accordance with APA guidelines (Wicherts et al. 2006). Tests of generalizability require broad access to published data.

Given the general state of ignorance with regard to the generalizability of so many findings, we think granting agencies, reviewers, and editors would be wise to give researchers credit for tapping and comparing diverse subject pools. Work with undergraduates and the children who live around universities is much easier than going out into the world to find subjects. As things stand, researchers suffer a competitive disadvantage when seeking a more diverse sampling of subjects. Because many of the best journals routinely require that papers include several studies to address concerns about internal validity (Carver 2004), the current incentives greatly favor targeting the easiest subject pool to access. There is an often unrecognized tradeoff between the experimental rigor of using multiple studies and the concomitant lack of generalizability that easy-to-run subject pools entail (Rozin 2009). If the incentive structure came to favor nonstudent subject pools, we anticipate that researchers could also be more persuasive in encouraging their universities and departments to invest in building non-student subject pools - for example, by setting up permanent psychological and behavioral testing facilities in bus terminals, Fijian villages, rail stations, airports, and anywhere diverse subjects might find themselves with extra time.

Beyond this, departments and universities should build research links to diverse subject pools. There are literally untapped billions of people around the world who would be willing to participate in research projects, as both paid subjects and research assistants. The amounts of money necessary to pay people who might normally make less than $\$ 12$ per day are trivial vis-à-vis the average research grant. Development economists, anthropologists, and public health researchers already do extensive research among diverse populations, and therefore already possess the contacts and collaborations. Experimentalists merely need to work on building the networks.

Funding agencies, departments, and universities can encourage and facilitate both professors and graduate students to work on expanding sample diversity. Research partnerships with non-WEIRD institutions can be established to further the goal of expanding and diversifying the empirical base of the behavioral sciences. By supplying research leaves, adjusted expectations of student progress, special funding sources, and institutionalized relationships to populations outside the university as well as to nonWEIRD universities, these organizations can make an important contribution to building a more complete understanding of human nature.

\section{Closing words}

Although we are certainly not the first to worry about the representativeness of prevalent undergraduate samples in the behavioral sciences (Gergen 1973; Medin \& Atran 2004; Norenzayan \& Heine 2005; Rozin 2001; 2009; Sears 1986; Sue 1999), our efforts to compile an empirical case have revealed an even more alarming situation than previously recognized. The sample of contemporary Western undergraduates that so overwhelms our database is not just an extraordinarily restricted sample of humanity; it is frequently a distinct outlier vis-à-vis other global samples. It may represent the worst population on which to base our understanding of Homo sapiens. Behavioral 
scientists now face a choice - they can either acknowledge that their findings in many domains cannot be generalized beyond this unusual subpopulation (and leave it at that), or they can begin to take the difficult steps to building a broader, richer, and better-grounded understanding of our species.

\section{ACKNOWLEDGMENTS}

We thank several anonymous reviewers and the following colleagues for their very helpful comments on earlier versions of this manuscript: Nicholas Epley, Alan Fiske, Simon Gächter, Jonathan Haidt, Shinobu Kitayama, Shaun Nichols, Richard Nisbett, Paul Rozin, Mark Schaller, Natalie Henrich, Daniel Fessler, Michael Gurven, Clark Barrett, Ted Slingerland, Rick Shweder, Mark Collard, Paul Bloom, Scott Atran, Doug Medin, Tage Rai, Ayse Uskul, Colin Camerer, Karen Wynn, Tim Wilson, and Stephen Stich.

\section{NOTES}

1. We also use the term "WEIRD" throughout this paper to refer to the exceptional nature of this sample, and do not intend any negative connotations or moral judgments by the acronym.

2. Key steps include: (1) establishing nationally representative experimental samples in Europe (Fehr et al. 2002; Guth et al. 2003); (2) applying experimental methods in developing countries (Cardenas \& Carpenter 2008; Tanaka et al., forthcoming); (3) creating university-wide subject recruiting rather than discipline-specific subject pools (most economic experiments); and (4) targeting specific samples of non-student subjects (Bellemare et al. 2008; Bellemare \& Kröger 2007; Harrison et al. 2002; List 2004).

3. Comparative studies of individual decision-making processes using samples from small-scale and WEIRD populations, including explorations of risk aversion, prospect theory, and inter-temporal choice, yield mixed results. Sometimes similarities, both qualitative and quantitative, are found. Other times differences emerge (Cardenas \& Carpenter 2008; Henrich \& McElreath 2002; Hsu et al. 2009; Humphrey \& Verschoor 2004a; 2004b; Kirby et al. 2002; Tanaka et al., forthcoming). So far, we do not see how to figure out which features will vary and which will not.

4. Rivers, for instance, found that cultures with a single color term for blue and green could still tell the difference between a blue and a green thread. (See Rivers 1901a).

5. Fessler also emphasizes important differences in shame and guilt between Americans and Indonesians.

6. To illustrate the limits of inferring universality from twopopulation comparisons, we note the finding that field independence on the Rod \& Frame test is shown for both migratory foragers and Americans (Witkin \& Berry 1975), yet East Asians and sedentary foragers show evidence for field dependence (Ji et al. 2000).

7. We are using "Western" to refer to those countries clustered in the northwest of Europe (the United Kingdom, France, Germany, Switzerland, the Netherlands, etc.), and British-descent societies such as the United States, Canada, New Zealand, and Australia. In particular, we are concerned about those populations from which most subjects in behavioral and psychological experiments are drawn. We recognize that there are important limitations and problems with this label, but we use it for convenience.

8. See also Knowles et al. (2001); but, for contrary findings, see Lieberman et al. (2005).

9. Interestingly, evidence indicates a somewhat different pattern in small-scale societies; see Marlowe (2004), Moore et al. (2006), and Pillsworth (2008).

10. Efforts to replicate these findings in various small-scale societies have all failed (Marlowe \& Wetsman 2001; Sugiyama
2004; Yu \& Shepard 1998). These failures suggest a more complicated and context-specific set of evolutionary hypotheses (Marlowe et al. 2005; Swami \& Tovée 2007).

11. The factor structure was less evident in a number of developing populations (e.g., Botswana, Ethiopia, Lebanon, Malaysia, Puerto Rico, Uganda), where independent assessments revealed that the data quality was poor. Future efforts to obtain betterquality data from these countries are important for demonstrating the universality of the Five Factor Model.

12. The robustness of the Five Factor Model is considerably weaker when it is derived from indigenous personality traits from other languages, although some of the five traits do still emerge (Benet-Martinez \& Waller 1995; Cheung et al. 1996; Saucier et al. 2005).

13. As American and Canadian researchers at a Canadian university, we note that Canada is also a highly unusual population along the same lines as the United States, although perhaps not quite as pronounced as the United States, at least in terms of individualism (Hofstede 1980).

14. These examples illustrate a parallel problem for those interested in the differences between human and nonhuman cognition. Since most ape-human comparisons involve WEIRD people (or their children) as subjects, some seeming apehuman differences may not represent real species-level contrasts, but may instead reflect the psychological peculiarities of WEIRD people (Boesch 2007).

15. Thanks to Shaun Nichols for pointing this out.

16. We note that the heuristics and biases derived from this empirical work were, however, readily extended to "people" without hesitation (Kahneman et al. 1982).

\section{Open Peer Commentary}

\section{Why a theory of human nature cannot be based on the distinction between universality and variability: Lessons from anthropology}

\author{
doi:10.1017/S0140525X10000026
}

\section{Rita Astuti and Maurice Bloch}

Department of Anthropology, London School of Economics and Political

Science, London WC2A 2AE, United Kingdom.

r.astuti@Ise.ac.uk

http://www2.Ise.ac.uk/anthropology/people/astuti.aspx

m.e.bloch@Ise.ac.uk

http://www2.lse.ac.uk/anthropology/people/bloch.aspx

Abstract: We welcome the critical appraisal of the database used by the behavioral sciences, but we suggest that the authors' differentiation between variable and universal features is ill conceived and that their categorization of non-WEIRD populations is misleading. We propose a different approach to comparative research, which takes population variability seriously and recognizes the methodological difficulties it engenders.

The authors of the target article, Henrich et al., call for an ambitious reorganization of the behavioral sciences, motivated by two key observations: (1) that the populations on which behavioral scientists typically base their findings are outliers from the rest of humankind; and (2) that there is significant population variability, which complicates the identification of those behavioral and psychological features that are universal. We start by appraising 
each of these observations (in reverse order), and we conclude by proposing a different approach to comparative research.

1. Population variability. The existence of significant population variability is convincingly documented by the authors, who find it as soon as they look beyond the ridiculously narrow samples on which claims of universality have typically been based. Although in agreement with the finding, we have a problem with the authors' underlying assumption. This is the idea that it is possible to neatly sort variable features of human behavior and psychology from universal ones. As anthropologists, we have no problem in accepting that cultural, historical, and environmental contexts affect all the features discussed in the article, but this observation has no bearing on the question of whether such features are "universal" or "variable." It is the variable/universal dichotomy itself (and the questions it generates) that is misleading. This is because human beings are affected simultaneously by processes of a different nature, among them phylogeny, history in its social and cultural instantiations, and ontogeny. But none of these processes is ever active in isolation, making it impossible to track its universal or variable effects. Searching in any human phenomenon for the clear signature of one of these processes in isolation is a wild goose chase.

2. WEIRD as outliers. The authors use three broad population contrasts in order to zoom in on the weirdness of the subject population used to generalize about human nature. The point is well taken, as is the call for more research among non-WEIRD populations. In their eagerness to condemn the reliance on WEIRD subjects, however, the authors end up presenting and conceptualizing population variability in terms of extremely dubious categories. Curiously, while they feel the need to clarify what they mean by the term "Western" and to acknowledge its limitations, they offer no apology for using "small-scale societies" as if the term referred to a unified, meaningful whole (a similar point could be made for "non-Westerner" or "East Asian"). This uncritical lumping together of a variety of disparate societies is particularly odd in a paper that denounces unsound generalizations. As clearly demonstrated by the results of the economic games, some "small-scale societies" can vary just as much among themselves as they do from the WEIRD population - a fact that should not be surprising given that "small-scale societies" are as caught up in the flow of human history as any other. One could argue that the extreme weirdness of the WEIRD population is partly the result of having lumped together other populations under too simplistic and under-theorized labels.

3. Our proposal. As anthropologists committed to the study of human nature (see Bloch 2005), we welcome Henrich et al.'s critical appraisal of the behavioral sciences' comparative database. We feel, nonetheless, that the authors have not sufficiently taken to heart the fundamental implications of their analysis. One obvious conclusion they might have drawn is that behavioral scientists should pay more attention to the work of cultural/ social anthropologists, since these are the scientists who have made human variability their main focus. It is striking, however, how little reference Henrich et al. make to anthropological research. This, of course, is no accident. It has to do with the kind of data that anthropologists have produced, which in turn has to do with the history of their discipline.

At the start, anthropologists went to the field with ready-made questions that were generated by a simplistic, yet highly influential, evolutionary theory, which is still the basis of popular understandings of the difference between "civilized" and "primitive" societies (the latter sometimes euphemistically called "smallscale"). But such outmoded theory had to be abandoned because, it was soon realized, human history does not proceed along a progressive and unilineal path. Because of the human capacity for culture, each human society is the unique product of a unique, albeit not isolated, history.

Ever since the recognition of this fact, anthropologists have faced a methodological difficulty: Questions formulated from within one historical context produce misleading answers when transposed elsewhere, as they appear weird, uninterpretable, or mean something else (arguably, this is what generates the weirdness of the WEIRD population, since what distinguishes it from all the others is that it is the one that generates the questions). The way anthropologists have tried to overcome this challenge has been to abandon, initially at least, all questions formulated outside the context under their investigation. Rather, through participant observation, they have allowed themselves to discover, from the inside, the terms and values of the people they study. This strategy is not without difficulties, as it generates a kind of data that appears impressionistic and anecdotal and which, crucially, precludes comparison and generalization which is why such data is so often ignored by other behavioral scientists, such as the authors of the target article. We recognize that this is a very serious limitation, but we insist that behavioral scientists must acknowledge and never underestimate the equally serious and unavoidable problem that led anthropologists down this methodological route in the first place.

Therefore, the solution cannot be, as suggested by Henrich et al., to administer studies upon studies to the billions of (poor) people around the world who remain untapped by the behavioral sciences. The solution is far more complicated and costly. It requires an often uncomfortable compromise between internal validity and generality, and a lot more detailed ethnographic work than many seem to be willing to accept (see, e.g., Astuti \& Harris 2008; Astuti et al. 2004). Only in this way will data from non-WEIRD populations become a meaningful and indispensable ingredient of any general theory about our species.

\section{Weird people, yes, but also weird experiments}

\section{doi:10.1017/S0140525X10000038}

\section{Nicolas Baumard ${ }^{\mathrm{a}}$ and Dan Sperber ${ }^{\mathrm{b}}$ \\ a Institute of Cognitive and Evolutionary Anthropology, University of Oxford, Oxford OX2 6PN, United Kingdom; ${ }^{\mathrm{b}}$ Institut Jean Nicod, Ecole Normale Supérieure, 75005 Paris, France. nbaumard@gmail.com \\ https://sites.google.com/site/nicolasbaumard/Home dan@sperber.fr http://www.dan.sperber.fr/}

Abstract: While we agree that the cultural imbalance in the recruitment of participants in psychology experiments is highly detrimental, we emphasize the need to complement this criticism with a warning about the "weirdness" of some cross-cultural studies showing seemingly deep cultural differences. We take the example of economic games and suggest that the variety of results observed in these games may not be due to deep psychological differences per se, but rather due to different interpretations of the situation.

Henrich et al.'s article fleshes out in a very useful and timely manner comments often heard but rarely published about the extraordinary cultural imbalance in the recruitment of participants in psychology experiments and the doubt this casts on generalization of findings from these "weird" samples to humans in general. The authors mention that one of the concerns they have met in defending their views has been of a methodological nature: "the observed variation across populations may be due to various methodological artifacts that arise from translating experiments across contexts" (sect. 7.2, para. 1). Here we want to express a less sweeping methodological concern. While accepting the general conclusions and recommendations of the article, we believe they should be complemented with a warning about the "weirdness" of some experimental designs that have been used across cultures and seem to show deep cultural differences. In fact, they may just show quite different interpretations of the experimental situation by the participants. This is not to deny, of course, that these differences in interpretations are themselves 
Commentary/Henrich et al.: The weirdest people in the world?

both psychological and cultural and are worth studying in their own right. In fact, unless one pays attention to them, it unclear what the experimental evidence is really about.

Let us illustrate our point with the case of economic games (discussed in sections 3.2, 4.1, and 6.1 of the target article). In these experiments, people are given a sum of money for free (which never happens in the real life) and have to share it with someone about whom they have no information (which also never happens in real life). Many researchers, including one of the article's authors (see Henrich et al. 2005), have pointed out that cultural variations in economic games may have more to do with methodological problems than with actual cultural differences (Ensminger 2002; Heintz 2005; Lesorogol 2007). In particular, participants in these games have no information about the rights of each player over the stake and are asked to make a "blind" decision. But who owns the money? Is the money a gift? Is the money a payment in exchange for my participation? Who is the other participant? Is he or she someone I know? Does he or she have rights over the money? And so on.

This leaves open the possibility that behavioural differences observed in economic games are not due to deep psychological differences per se, but rather due to different interpretations of the situation (for a similar point, see Hagen \& Hammerstein 2006; Heintz 2005). For example, Henrich et al.'s (2005) study in 15 small-scale societies reveals a striking difference between the Lamalera, who make very generous offers in the Ultimatum Game, and the Tsimane and the Machigenga, who make very low offers in the very same game. But the game is likely to be construed very differently within these societies. The Lamalera, being collective hunters, may indeed see the money as jointly owned by the proposer and the recipient. By contrast, the Tsimane and the Machigenga, who are solitary horticulturalists, may see the money as their own property and therefore feel entitled to keep it. In the same way, Westerners may appear as outliers not because they have a different moral psychology, but rather because, living in very large, democratic and capitalist societies, they make different assumptions in economic games (e.g., that, not knowing the other participant - a situation of anonymity that is common in large-scale urban societies - they have no particular duty to share the stake with her).

In line with this idea, economic games framed within a more detailed context tend to show that people's decisions are based on property rights (Oxoby \& Spraggon 2008), past contributions to collective actions (Cappelen et al. 2007; Frohlich et al. 2004), or a personal link of solidarity (Cronk 2007). One possible interpretation is that participants try to be fair with others when they distribute the money: If the other player has produced the money, she has more right over it; if she has been more productive or has invested more money, she deserves a bigger part of it; if both players are friends, they have special duties toward each other; and so forth. Such a "sense of fairness" combined with contextual differences might well explain the variety of results observed around the world. When confronted with cultural differences in experimental result, we should therefore ask: Are they the product of deep differences in the psychological dispositions and processes these experiments are intended to illuminate, or do they reflect differences in the interpretation of the experimental situation? One way to help answer this question would be, for instance, to present the Lamalera and the Machigenga with, as much as possible, the same rich context (e.g., clarifying the source of the money and the relationships between the participants) and assess whether they use the parameters at stake (i.e., rights, past contributions, social links) in the same way.

The importance of the way participants interpret a task - which may differ from the way the experimenter intended them to interpret it - has been often stressed in experimental psychology (e.g., Sperber et al. 1995). The more the experiment is artificial and devoid of "ecological validity" - in other terms, the weirder it is the greater the risk of misinterpreting the differences between societies. When it comes to cross-cultural comparisons, ignoring this pragmatic dimension of participants' performance may cause one to exaggerate or to miss genuine psychological differences.

\section{Weirdness is in the eye of the beholder}

\author{
doi:10.1017/S0140525X1000004X
}

\section{Will M. Bennis and Douglas L. Medin \\ Department of Psychology, Northwestern University, Evanston, IL 60208. \\ wbennis@northwestern.edu \\ medin@northwestern.edu \\ http://www.wcas.northwestern.edu/psych/people/faculty/ \\ faculty_individual_pages/Medin.htm}

Abstract: Henrich et al.'s critical review demonstrating that psychology research is over-reliant on WEIRD samples is an important contribution to the field. Their stronger claim that "WEIRD subjects are particularly unusual" is less convincing, however. We argue that WEIRD people's apparent distinct weirdness is a methodological side-effect of psychology's over-reliance on WEIRD populations for developing its methods and theoretical constructs.

In their important article, Henrich et al. offer both weak and strong versions of an argument against the widespread use of research with WEIRD (Western, Educated, Industrialized, Rich, and Democratic) people as a means to learn about general human psychology. The weak version critiques the over-reliance on such samples and reviews an extensive body of literature across domains to establish that widespread cross-cultural differences exist for many of the psychological findings researchers have assumed were species universal. We are one hundred percent convinced of the weak argument and strongly endorse its attendant plea for moving beyond WEIRD samples. Their review is a major contribution to the literature, and we thank the authors for it.

The strong version of the argument makes the additional point that WEIRD people are literally weird, atypical of humankind at large. On this account, it is the field's ironic misfortune that of all samples to study, psychology should have picked this one. This strong argument is intriguing, and Henrich et al. present extensive evidence suggesting that this narrow slice of humanity indeed is a cultural outlier. For reasons that form the basis of our commentary, however, we remain skeptical with respect to this strong argument.

The evidence for the distinctness of WEIRD samples comes from studies that generally take the following form: Findings originally conducted on the WEIRD population are assessed with a different population, and a different pattern of results emerges. When a broader range of groups is considered, the WEIRD population tends to be at the extreme in its responses. For example, Henrich et al. cite Segall and colleagues' replication of the well-known Müller-Lyer illusion (Segall et al. 1996). Segall et al. find not only a wide distribution of the magnitude of the illusion across cultures, but also that the U.S. sample is the most extreme in magnitude (see their Fig. 1). Other phenomena they review demonstrate a similar trend.

Base rates provide one clue that there might be something amiss with the argument that the group with which we are most intimately familiar is also the most distinctive. If there are a thousand potential samples, then the probability that the first selected is the most deviant is one out of a thousand.

We think the apparent extremity of WEIRD populations can best be explained by two factors contributing to what we have called "the home-field disadvantage" - that is, the tendency for research developed in one's "home-culture" and subsequently co-opted for cross-cultural comparison to result in one-sided conclusions about the nature of cross-cultural differences (Medin et al., under review).

The first factor is the similarity between researcher and researched. Variations across cultures may reflect both adaptations to particular environments (e.g., Nisbett \& Cohen 1996) and "niche 
construction" (environmental adaptations that favor and reinforce cultural characteristics; Laland et al. 2000). For example, it may not matter whether people drive on the left or right side of roads; but once there is a consensus in a given culture, it is adaptive to conform to it. Researchers may have privileged insight in their own culture into what is important or what experimental manipulations are likely to achieve interesting and reliable results, and they may find it natural to study these sorts of things. But the very fact that the results are important, interesting, or reliable in one's home culture makes it more likely that one's culture represents an extreme with respect to those results (see Medin \& Bang 2008).

The other main factor reinforcing apparent extremes among WEIRD samples is their status as the originating research population. Research methods and theoretical constructs are calibrated to the populations they have been selected and designed for: in psychology's case, WEIRD people. A side-effect is that these same tools are less well fit or even ill fit to other populations, in much the same way that any adaptation evolved for a particular niche will not function as well in other niches. For example, imagine a literature on sense of humor evolved from studies with undergraduates at major U.S. universities. Jokes that proved to be effective would tend to appear in later studies and ones that fell flat would tend to go by the wayside. If one then got the bright idea of doing a cross-cultural comparison, it may seem natural to use the same jokes favored by U.S. college students, with the more or less inevitable consequence that other populations wouldn't find these jokes quite so funny, and the U.S. college sample would appear to be an extreme.

Consider the Müller-Lyer illusion mentioned above and discussed in the target article. That particular illusion is a classic of Western psychology, taught in any introductory class discussing perceptual illusions. And it is taught because it is so readily demonstrated, a fact that reveals both general properties of the perceptual system and a response to the perceptual environment in which Westerners live. Small wonder that the effect is weaker in populations exposed to a different perceptual environment. Similarly, some novel perceptual illusion discovered in some other population is likely to be smaller in magnitude when tested with our WEIRD sample. But that is just our point - overwhelmingly, psychological research originates with the WEIRD sample and then is applied elsewhere - the converse pattern is rare. We believe that this habit of using research methods and theoretical constructs (stimuli, procedures, models, etc.) for cross-cultural comparisons that originated with WEIRD samples, coupled with insider information about what those WEIRD samples find important and which experimental manipulations are likely to achieve interesting and reliable results, may well account for the apparent extremity of the WEIRD population. Had psychology started with Chinese rice farmers studying members of their own community and then later their research protocols and theoretical constructs were exported for cross-cultural comparison and tested for universal validity, then, on our account, Chinese rice farmers would be the cultural outliers and WEIRD people would look more like everyone else.

\section{Away from ethnocentrism and anthropocentrism: Towards a scientific understanding of "what makes us human"}

\section{doi:10.1017/S0140525X10000051}

\section{Christophe Boesch \\ Department of Primatology, Max Planck Institute for Evolutionary Anthropology, 04103 Leipzig, Germany. \\ boesch@eva.mpg.de \\ http://www.eva.mpg.de/primat/staff/boesch/index.html}

Abstract: The quest to understand "what makes us human" has been heading towards an impasse, when comparative psychology compares primarily individuals that are not representative of their species. Captives experience such divergent socioecological niches that they cannot stand for their wild counterparts. Only after removing ethnocentrism and anthropocentrism will we be able to progress in our understanding of "what makes us human."

Henrich et al.'s review of cognitive differences among human cultures is very timely in reminding us that different living conditions have consequences for cognitive development. Not all humans are Westerners, and this is true also for their cognition. Here, I want to address how this affects our understanding of cognitive differences between humans and chimpanzees, and requires reconsideration of many claims about "what makes us human" (Boesch 2007; 2008). To become a science, comparative psychology will have to include population differences in its theoretical thinking and empirical approaches.

Comparative psychology suffers from the same weaknesses as noted by Henrich et al. for psychology. Bold claims about "human uniqueness" are made based on the assumption that WEIRD (Western, Educated, Industrialized, Rich, and Democratic) societies' humans (I referred to them as WMC, or Western middle class, humans; Boesch 2007) and captive chimpanzee populations are representative of each of the two species (Boesch 2007; de Waal 2001). Comparative psychology predominantly compared captive chimpanzees with free Western humans (see black arrow A in my Figure 1). The overwhelming conclusion of these studies was that humans clearly outperform chimpanzees in such different cognitive domains as folk physics, altruism, cooperation, theory of mind, and gaze following (e.g., Hermann et al. 2007; Povinelli 2000; Povinelli \& Vonk 2003; Silk et al. 2005; Tomasello et al. 2005).

However, three essential points that invalidate their conclusions have been too often forgotten in the interpretation of such studies. First, the characteristics of the animal populations included in these studies are typically not representative of their species. The Louisiana captive chimpanzee group that has been used extensively in a variety of widely cited cognitive studies (e.g., Povinelli 2000; Povinelli \& Vonk 2003; Silk et al. 2005; Vonk et al. 2008) nicely illustrates this point (see Fig. 1). This group was created by putting together seven 2- to 3-year-old chimpanzees that were kept in isolation as a same-aged peers group all their lives, in a small, stable, and restricted man-made environment (see Povinelli 2000). Such a history cannot be more different from the one of young chimpanzees in the wild. Wild individuals live in large, flexible, fission-fusion groups, with 30 to 100 individuals of different

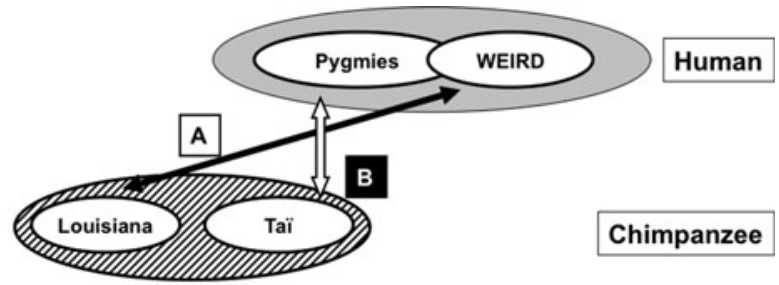

Socio-ecological niche

Figure 1 (Boesch). Schematic representation of the cognitive landscape in humans and chimpanzees as a function of the different socioecological niches that each species encounters. For each species, the possible range of cognitive performance is illustrated by an ellipse including all the individual population performances. Two types of cross-species comparisons are illustrated: The first one, the classical comparative psychology approach, compares two outlier populations for their species (black arrow A compares captive chimpanzees with WEIRD humans); and the second one compares populations of two species facing similar socioecological niches (white arrow B). 
Commentary/Henrich et al.: The weirdest people in the world?

ages, in kilometer-wide ranges where food has to be located and extracted, and where life-threatening neighbors and predators loom (e.g., Mitani et al. 2002). True, captive conditions range from highly ecologically deprived environments, typical for the early $1950 \mathrm{~s}$, to much more enriched conditions, as seen in modern settings. Moreover, social conditions vary from complete isolation to more social groups. However, all captive conditions differ from wild ones in that captives are forced to live in much smaller, stable social groups, in very passive environments where food is provided and no competition with others exists. The fields of developmental and social psychology have shown that such differences have important effects on cognitive development in humans (Carpendale \& Lewis 2004; Nelson et al. 2007).

No surprise that some captive chimpanzees have difficulties in understanding unseen relationships (Povinelli 2000), whereas wild chimpanzees transport stone hammers to distant, out-ofsight nut-producing trees (Boesch \& Boesch 1984) and use tools to extract unseen underground resources (Boesch et al. 2009; Sanz et al. 2004). Similarly, some captives are unable to share food (Silk et al. 2005; Warneken \& Tomasello 2006) or to work as a team with shared goals (Tomasello et al. 2005), whereas wild individuals share vast quantities of food with unrelated group members for extended periods of time and work as a close team when hunting prey, chasing leopards, or during risky intergroup encounters (e.g., Boesch \& Boesch 1989; Boesch et al. 2008; 2010; Goodall 1986; Mitani \& Watts 2005).

Second, comparative psychology has favored experimental studies using anthropocentric designs and assumptions. These might allow testing human abilities in other species, but are unlikely to uncover cognitive abilities of nonhuman animals. For example, to understand the altruistic abilities of chimpanzees, experiments have been designed on the ethnocentric assumption that sharing should be preferred over nonsharing when there is no cost to oneself (Silk et al. 2005). However, sharing implies a contractual obligation in some human populations (Henrich et al. 2006; and present study in the target article); and, therefore, this assumption does not even hold in all human populations. Similarly, numerous experiments with captive chimpanzees used a WEIRD notion of causality. In gaze-following experiments, tested animals needed to understand that a human gaze indicated an honest positive interest (Hermann et al. 2007; Tomasello et al. 2005). In helping experiments, tested individuals had to understand that experimenters pretending to not master a task needed to be helped (Warneken \& Tomasello 2006). Less ethnocentric and anthropocentric experiments would bring us a long way to understand other species.

Third, by favoring experiments in captive settings, comparative psychology has opted for low ecological validity. For example, altruism in wild chimpanzees is expressed mainly in situations where a highly sought after food, meat, is shared with individuals that are socially important to the giver, either because they are hunting partners or social allies (Boesch 2009; Mitani \& Watts 2001). Such a social dimension has rarely been considered in comparative experiments. Similarly, chimpanzees primarily cooperate during life-threatening situations, such as during intergroup fights or when predators are near, or to get meat (Boesch 2009; Goodall 1986). The difficulties with mimicking such situations in experiments have not prevented comparative psychologists from making strong claims about chimpanzees' limitations (Herrmann et al. 2007; Povinelli 2000; Tomasello et al. 2005). Comparative psychologists' inability to mimic natural cooperative conditions is not proof that cooperative ability is absent in other animal species.

The quest to understand "what makes us human" has been heading towards an impasse. It will progress again once the socioecological diversity of humans and other species are considered. I am asking for greater care before making sweeping claims based on only a few captive individuals. Knowing that cognitive diversity is natural in species living in different socioecological conditions, we need to compare what is comparable (following white arrow B in Figure 1). Only when this condition is met will our quest to understand "what makes us human" progress.

\section{The WEIRD are even weirder than you think: Diversifying contexts is as important as diversifying samples}

\author{
doi:10.1017/S0140525X10000063
}

Stephen J. Ceci, ${ }^{a}$ Dan M. Kahan, ${ }^{b}$ and Donald Braman ${ }^{\mathrm{C}}$

${ }^{a}$ Department of Human Development, Cornell University, Ithaca, NY 14853;

${ }^{\mathrm{b}}$ Yale Law School, Yale University, New Haven, CT 06520-8215; ' George

Washington University Law School, Washington, DC 20052.

Sjc9@cornell.edu

http://www.human.cornell.edu/che/bio.cfm?netid = sjc9

dan.kahan@yale.edu

http://www.culturalcognition.net/kahan/

donald.braman@gmail.com

http://www.culturalcognition.net/braman/

Abstract: We argue that Henrich et al. do not go far enough in their critique: Sample diversification, while important, will not lead to the detection of generalizable principles. For that it will be necessary to broaden the range of contexts in which data are gathered. We demonstrate the power of contexts to alter results even in the presence of sample diversification.

We commend Henrich et al. for pointing out the (over)reliance on participants from WEIRD societies and the tenuousness of universal laws whose support is based on a single subpopulation of a single society. Notwithstanding the merit of studying participants from diverse races, social classes, and national cultures, this will not in itself lead to generalizability of findings because it leaves unaddressed other threats to generalization, including the restricted physical, ideological, and attitudinal parameters of most research, and the omission of social meanings that participants attach to their choices.

Even when researchers include non-WEIRD participants, they rarely include contextual variation. Few of our principles are based on data from diverse settings and conditions. On those occasions when researchers do insert contextual diversity into their designs, it becomes apparent that theorizing is paradigm-bound - confined to the specific physical, motivational, and psychological conditions under which the data were gathered. Below we argue for the power of manipulating the context and social meanings, independent of sample diversity.

Motivational context. When researchers contrast paradigms across settings, stimuli, and/or conditions, the results sometimes fail to replicate. For example, Ceci and Bronfenbrenner (1991, cited in Ceci 1996) asked children to predict where on a monitor geometric shapes would migrate after children pressed the space bar. A curvilinear algorithm determined where each shape would migrate $^{1}$ :

$$
.8 \sin (\mathrm{x})+.6 \sin (\mathrm{y})+.4 \sin (\mathrm{z})+5 \% \text { error }
$$

Even after 750 trials, children were still unable to predict the shapes' migration. The implication is that multiplicative reasoning is beyond their capability.

As shown in our Figure 1, however, when the identical algorithm controlled a video game in which the object was to predict the destination at which vehicles would meet a roadblock, children reached ceiling by 450 trials (Ceci 1996). Thus, behavior in ecologically challenging contexts led to findings at odds with those from socially sanitized settings.

Semantic context. Much research on memory, reasoning, and moral development is based on stimuli expunged of meaningful associations (e.g., nonsense syllables) in the belief this will reveal underlying principles. For example, Wason's deduction 


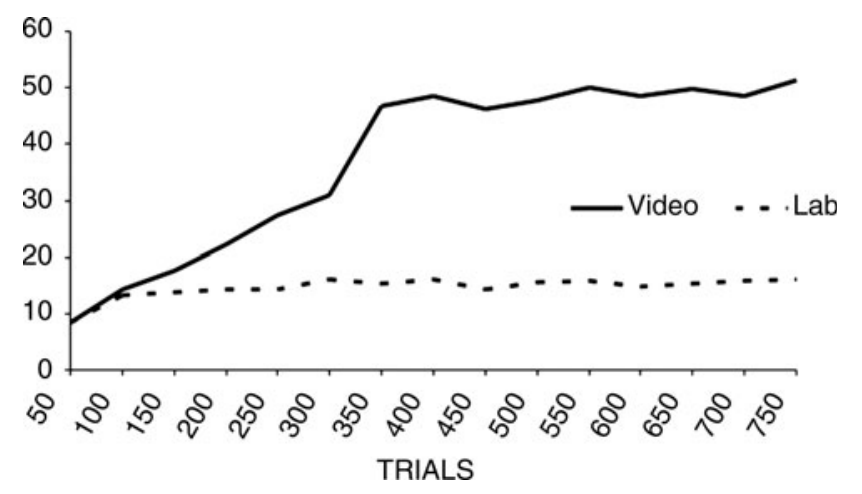

Figure 1 (Ceci et al.). Curvilinear Distance Estimation in and out of Laboratory.

task has been used to illuminate qualities of thinking. However, even when participants were from other cultures, it was clear that deduction depended on the content. Consider: The logic underlying Wason's task is that any inference that has the form:

$$
\begin{aligned}
& \mathrm{p} \text { or } \mathrm{q} \\
& \text { not- } \mathrm{p} \\
& \therefore \mathrm{q}
\end{aligned}
$$

is valid independent of the propositional content. However, it has been repeatedly shown this is not true and that content matters. Suppose four cards are laid out

\section{E K 47}

with the instructions that each card has a number on one side and a letter on the other. Participants are told: If $a$ card has a vowel on one side, then it has an even number on the other side. Their job is to determine which cards must be turned over to validate the rule. Even smart undergraduates have success rates of only $12 \%$. However, if the content is changed to a mode of transportation on one side of each card and a destination on the other

\section{Manchester Sheffield Train Car}

and the rule is: Every time I go to Manchester, I go by train. Nothing has changed logically, but performance skyrockets to $60 \%$ (Johnson-Laird 1983). Similarly, we have demonstrated that differences in the way meaning is represented mediate children's metacognition (Ceci et al. 2010).

Cultural-cognitive context. Two of us (Kahan, Braman) have demonstrated that Americans who vary in their attitudes toward hierarchy and equality vary in their perceptions of legally consequential facts (Kahan, in press; Kahan et al. 2009). These competing attitudes cohere with opposing sets of norms, and related scripts of acceptable behavior, which can trump the demographic variables emphasized by the WEIRD critique (Kahan et al. 2007).

Social meaning context. Actions have meanings as well as consequences. They embody attitudes, the expression of which shapes actors' perceptions of what they are doing and hence the value of doing it. For example, under what circumstances will an actor prefer the certainty of one sum to the probability of another? "When the expected value of the former exceeds the latter," the classical microeconomist says, "subject to one's degree of risk aversion," which the behavioral economist notes will vary depending on whether one understands the exchange as realizing a "gain" as opposed to avoiding a "loss." But experimental work shows the answer also depends on what such an exchange means. Most members of WEIRD societies will shun trades involving tax evasion that they might well have accepted in the form of casino wagers, because the former, even if equivalent to the latter along the dimensions specified by economists, manifests attitudes and values antithetical to the self-conception of (most) WEIRD people (Baldry 1986). Likewise, economists' considerations - dominant payoff strategies, signaling, reciprocity - don't tell us all we must know to predict whether individuals will contribute to public goods (Liberman et al. 2004). Even the bedrock axiom of economics "demand curves slope downward" - founders on the shoals of meaning. Offering a wage can cause persons who previously did community service for free to refrain from doing any: payment destroys the meaning, and thus the associated value, of public-spirited behavior (Gneezy \& Rustichini 2000).

In a nutshell, ignoring meaning - as behavioral economics and related approaches do - generates unreliable predictions for any collection of persons who experience a common life. Heinrich et al. rightly question whether the behavioral dynamics in the work they examine generalize to non-WEIRD samples. But it is important not to assume this critique identifies some distinctive problem hovering at the "margins" of that work. Indeed, what does generalize about the new behavioral science is the question about its external validity: its neglect of meaning and other elements of context gives us reason to be cautious about accepting extrapolations from its stylized experiments.

In sum, our argument is that although we agree with Henrich et al. on the need to diversify samples, this alone will not unearth generalizable principles because contexts, attitudes, and meanings contribute systematic variance that must be included to reveal lawful ecological contrasts.

NOTE

1. The mapping function (over a quarter sine phase) was $x_{1} y_{1}$ $\mathrm{z}_{1}=$ random number $(0-9)$, where $\mathrm{x}, \mathrm{y}, \mathrm{z}=$ maxdistance $+1.8(0.8 \sin$ $\left.\mathrm{x}_{1} \times .10+0.60 \sin \mathrm{y}_{1} \times .10+0.4 \sin \mathrm{z}_{1} \times .10\right)$.

\section{The weirdest brains in the world}

\section{doi:10.1017/S0140525X10000282}

Joan Y. Chiao and Bobby K. Cheon

Department of Psychology, Northwestern University, Evanston, IL 60208.

jchiao@northwestern.edu

bobbycheon2012@u.northwestern.edu

http://culturalneuro.psych.northwestern.edu/Lab_Website/

Welcome.html

Abstract: Henrich et al. provide a compelling argument about a bias in the behavioral sciences to study human behavior primarily in WEIRD populations. Here we argue that brain scientists are susceptible to similar biases, sampling primarily from WEIRD populations; and we discuss recent evidence from cultural neuroscience demonstrating the importance and viability of investigating culture across multiple levels of analysis.

Henrich et al. provide a compelling argument regarding the error of assuming minimal variability across human populations in the behavioral sciences and the notion that people from WEIRD populations are actually unusual, even outliers, relative to the rest of the species. Here we argue that these problems of prematurely assuming universalism and experimentally sampling primarily from WEIRD populations extend beyond the behavioral sciences, into the brain sciences, and that researchers in both the behavioral and brain sciences may simultaneously benefit from reorganizing research infrastructures to promote the study of diverse cultural comparisons across multiple levels of analysis. 
Brain scientists generalize from a narrow sample to the species. Akin to the behavioral sciences, brain sciences typically sample from a thin slice of the species. Within the field of psychology, 95\% of psychological samples come from countries with only $12 \%$ of the world's population (Arnett 2008). Similarly, within the field of human neuroimaging, $90 \%$ of peer-reviewed neuroimaging studies come from Western countries (Chiao 2009).

Several factors have contributed to the current WEIRD sampling bias in the brain sciences. First, human neuroscience research programs typically build on either empirical questions inspired by animal models, or case studies of brain damaged patients, or theories from evolutionary psychology. Each of these three starting points for neuroscience research carries implicit assumptions of minimal variability across human populations. Second, researchers have lacked the technology to study culture at the neural level in humans, as human neuroimaging methods have become available only within the past three decades and are still not available in many non-Western regions of the world. The use of neuroimaging is often prohibitively expensive, making it easier for richer, politically stable countries, such as Western industrialized nations, to create the powerful societal infrastructures necessary for novel and timely neuroscientific discovery. The infrastructural advantages afforded to neuroscientists from WEIRD nations create researcher biases in the field that are cyclical and that compound over time as researchers create new experiments and seek empirical evidence consistent with theory and predictions based on prior findings. Hence, our current state of knowledge of mind-brain mappings is largely restricted to scientific observations made from people living within WEIRD nations, leaving a large empirical gap in our understanding of how diverse cultures affect mind, brain, and behavior.

It is not safe for brain scientists to generalize from a narrow sample to the species. In the target article, the authors identify key cultural comparisons that reveal behavioral differences across cultures, including industrialized versus small-scale societies, Western versus non-Western cultures, contemporary American versus non-American Westerners, and contemporary Americans versus other Americans. To the extent that behavior arises from neurobiological processes, it is plausible that this set of cultural comparisons would similarly reveal cultural differences in neural functioning.

Indeed, early efforts by cultural neuroscientists to address the question of how culture influences brain function have proven fruitful, particularly for understanding differences in neurobiological processing between Westerners and East Asians. Westerners engage brain regions associated with object processing to a greater extent relative to East Asians, who are less likely to focus exclusively on objects within a complex visual scene (Gutchess et al. 2006). Westerners show differences in medial prefrontal activity when thinking about themselves relative to close others, but East Asians do not (Zhu et al. 2007). Activations in frontal and parietal regions associated with attentional control show greater response when Westerners and East Asians are engaged in culturally preferred judgments (Hedden et al. 2008). Even evolutionarily ancient limbic regions, such as the human amygdala, respond preferentially to fearful faces of one's own cultural group (Chiao et al. 2008, Fig. 1). Taken together, these findings show cultural differences in brain functioning across a wide variety of psychological domains and demonstrate the importance of comparing, rather than generalizing, between Westerners and East Asians at a neural level.

What about cultural differences in brain function beyond comparisons of Westerners and East Asians? Critically, brain scientists have yet to explore the neurobiological ramifications of cultural contrasts beyond Westerners and East Asians. No research to date has explored comparisons in neural functioning of individuals living in small-scale versus industrialized societies. Additionally, although neuroimaging data do exist for comparing the neural functioning of contemporary Americans with that of other Westerners, such as Europeans, no study to our knowledge has yet explored in a hypothesis-driven manner the possibility that brain-behavior relations may vary as a function of the kind of Western subculture. Social neuroscientists have recently begun to address how childhood socioeconomic status affects brain functioning (Hackman \& Farah 2009); however, the effect of education level (e.g., college vs. no college education) on neural functioning remains unknown. Hence, the current state of knowledge in cultural neuroscience remains woefully behind that of cultural psychology. Yet, the knowledge to date supports the notion that it is not always safe for brain scientists
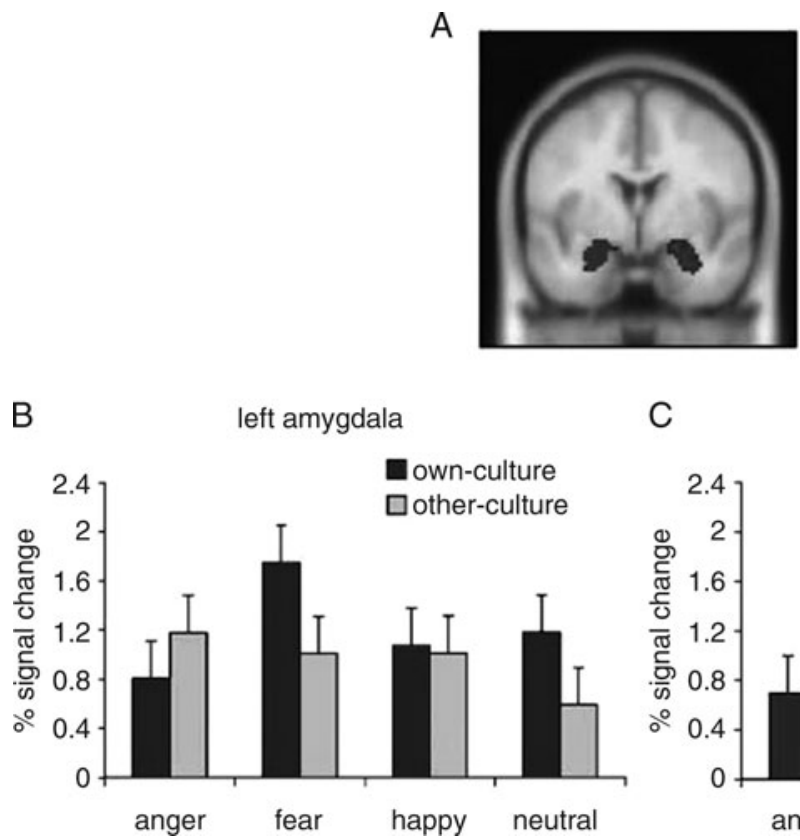

\section{C right amygdala}

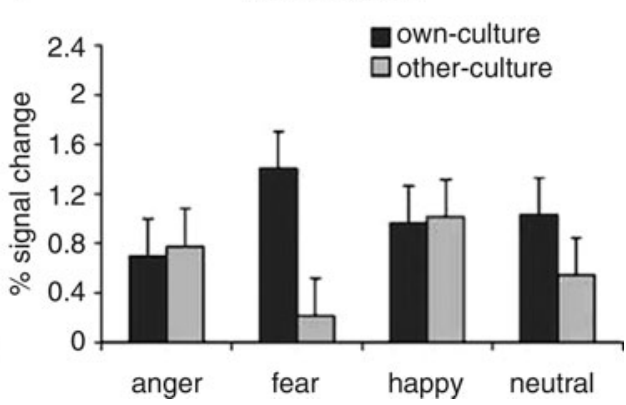

Figure 1 (Chiao \& Cheon). Greater bilateral amygdala response to own-culture relative to other-culture fear faces (from Chiao et al. 2008). 
to generalize from a narrow sample to the species and that restricting neuroscientific study to WEIRD populations will not be sufficient for building a comprehensive understanding of human nature.

Behavioral scientists and brain scientists alike will mutually benefit from studying non-WEIRD populations. The journey towards understanding how culture affects human nature is one that behavioral and brain scientists can readily embark on together, and likely with mutual benefits. For instance, where do these cultural differences in brain and behavior come from? One possibility is that psychological and neural diversity comes from differential experience specific to a given culture. Another possibility is that diversity in brain and behavior results from culture-gene coevolution, whereby culture coevolves with genes that regulate endophenotypes, such as brain and behavior. Testing both explanations requires empirical work that examines multiple levels of analysis (e.g., gene, brain, mind, culture) across multiple time scales (e.g., situation, ontogeny, phylogeny).

It is humbling to think of the sheer volume of work that lies ahead to address these timely questions, but by working together, behavioral and brain scientists can offer each other a more complete cultural science than either can construct alone.

\section{Diversity in representations; uniformity in learning}

\section{doi:10.1017/S0140525X10000075}

\section{David Danks and David Rose}

Department of Philosophy, Carnegie Mellon University and Institute for Human and Machine Cognition, Pittsburgh, PA 15213.

ddanks@cmu.eduｄavidros@andrew.cmu.edu

http://www.hss.cmu.edu/philosophy/faculty-danks.php

Abstract: Henrich et al.'s conclusion that psychologists ought not assume uniformity of psychological phenomena depends on their descriptive claim that there is no pattern to the great diversity in psychological phenomena. We argue that there is a pattern: uniformity of learning processes (broadly construed), and diversity of (some) mental contents (broadly construed).

Henrich et al. argue - correctly, in our view - that there is great diversity both across and within domains of human cognition. They then argue for the stronger claim that there is no underlying rhyme or reason for this diversity. This latter claim grounds their central methodological point: For all (interesting and not obviously culturally induced) cognitive phenomena, psychologists ought not automatically conclude, from samples of Western undergraduates, that any particular cognitive phenomenon is uniform across people. This methodological conclusion obviously depends crucially on the stronger descriptive claim. If a pattern of uniformity emerged with respect to a particular type of cognitive phenomenon, then psychologists could justifiably generalize (in only those particular domains, of course). We argue here that such a pattern does emerge, if one carves up the space of cognitive phenomena appropriately. More precisely, we suggest that there is diversity in cognitive "contents" (broadly construed), but uniformity in learning "processes" (broadly construed).

Cognitive "phenomena" do not form a unitary type, as there is a natural, defensible distinction between the cognitive "objects" of the mind, and dynamic mental "processes." Cognitive objects include representations, knowledge structures, and so on. Mental processes influence both those cognitive objects, and also other processes. As one example of this distinction, there is a natural difference between a concept and the processes by which one learns that concept. Concepts are particular representations that can be deployed in a range of circumstances; concept learning processes are the means by which those representations emerge. Importantly, the word process in our argument should explicitly not be read as a synonym for "specific causal mechanism"; the distinction between a process and its "target" requires only a thin notion of "process" (i.e., something like "if-then" rules, not a specific neural mechanism). Furthermore, our argument does not depend on the particular ways in which learning processes are instantiated in different individuals, as long as those processes have the same computational/psychological profile.

We have no doubt that Henrich et al. are correct that there is great diversity in representations, including people’s folk-biological concepts, notions of "fairness" and "agency," and moral concepts. It is unsurprising that people who develop in different environments - physical, mental, or social - develop different representations of those environments. But diversity in representation does not imply diversity in learning processes. For example, diversity in our learned concepts is arguably due to differences in inputs, not differences in concept learning processes. After all, the whole point of learning is that the same process can produce very different outputs depending on the environment, and thereby enable the organism to adapt to the demands of an unpredictable or ever-changing environment. There are thus prima facie reasons - genetic and evolutionary to think that there is likely to be uniformity in learning processes. Of course, part of the point of Henrich et al.'s article is that we ought not rest with the assertion that "there really ought to be uniformity in learning processes"; instead, it is an empirical matter as to whether there actually is such uniformity.

Determining whether learning processes are uniform across both WEIRD and non-WEIRD individuals requires discovering the learning processes in each individual, which is inevitably a tricky matter. We can rarely (if ever) directly observe a learning process; instead, we must find its characteristics by determining how the relevant cognitive objects change in response to different inputs (either within- or between-participants). Despite these difficulties, it is nonetheless often possible to learn about different individuals' learning processes. Unfortunately, as Henrich et al.'s descriptive survey shows, there have been relatively few cross-cultural studies of learning processes, as opposed to representations or (relatively) automatic processing. By our reading, essentially all of the phenomena that Henrich et al. discuss are representations/contents, rather than learning processes. There are many psychological studies of learning processes, but almost entirely restricted to WEIRD participants. We must therefore look to more indirect evidence.

It is suggestive that many of the cases of cognitive uniformity that Henrich et al. identify are also cases in which the environment is plausibly (relatively) uniform. On the "uniformity in learning processes" view we advocate, one would predict this sort of representational uniformity just when there is relative uniformity in the relevant environment. For example, it is (in our view) unsurprising that the ability to pass the False Belief Task emerges across cultures, since (presumably) all developing individuals have social environments that require interaction with (and prediction of the behavior of) individuals with false beliefs.

Moreover, Henrich et al. themselves seem to assume uniformity of learning processes in some of their explanations for diversity. For example, they seem to endorse an explanation of diversity in the Müller-Lyer illusion in terms of variation in the visual environments of developing individuals. That is, Henrich et al. seem to argue that differences in adult visual representations are due to differences in infant/child visual environments. But implicit in this argument is an assumption that there is an "infant environment $\rightarrow$ adult visual representation" mapping that is shared between both WEIRD and non-WEIRD individuals. More generally, all explanations of diversity in terms of environmental variation presuppose that there is relative uniformity in learning processes (in our thin sense). Explanations based in environmental variation require some shared process by which that variation can lead to behavioral or cognitive differences. If there is no shared learning process, then an appeal to environmental differences is simply a non sequitur. 
Learning processes (in a thin sense) are different from the objects they influence, and this distinction can provide a basis for when to expect uniformity and diversity in human cognition. We suggest that uniformity should be expected for learning processes, and diversity should be expected for objects whenever the relevant learning environments differ (and there are no relevant, direct selection pressures on the objects). We do not doubt that people are more diverse than contemporary cognitive psychology admits, and Henrich et al. do a great service in providing a descriptive characterization of diversity. They simply paint with too broad a methodological brush.

\section{ACKNOWLEDGMENT}

David Danks is partially supported by a James S. McDonnell Foundation Scholar Award.

\section{Getting beyond the "convenience sample" in research on early cognitive development}

doi:10.1017/S0140525X10000294

\section{Anne Fernald}

\section{Department of Psychology, Stanford University, Stanford, CA 94305. afernald@stanford.edu}

Abstract: Research on the early development of fundamental cognitive and language capacities has focused almost exclusively on infants from middle-class families, excluding children living in poverty who may experience less cognitive stimulation in the first years of life. Ignoring such differences limits our ability to discover the potentially powerful contributions of environmental support to the ontogeny of cognitive and language abilities.

Arnett (2008) blames the narrowness of American behavioral research on a philosophy of science that focuses on universals in human cognitive and social psychology, ignoring variability and the factors that contribute to differences among people. Henrich et al. share the concern that researchers in these areas simply assume their findings are universal, but suggest that studies with children may provide more convincing evidence. I do not think that developmental psychologists should be let off the hook so easily. If the WEIRD (Western, Educated, Rich, Industrialized, and Democratic) sample that is studied so extensively in psychological research with adults consists of a privileged subset of $5 \%$ of the world's population, then the children represented in the burgeoning literatures on "core knowledge" (Spelke \& Kinzler 2007) and early language acquisition (Fernald \& Marchman 2006) are drawn from an even smaller sliver of affluent and highly educated families. Parents with the time, resources, and motivation to bring their infant to participate in a developmental study at a university laboratory are demographically even less diverse than the college students who predominate in studies with adults.

Why does this matter? Because differences in socioeconomic status (SES) are robustly associated with the quantity and quality of early cognitive stimulation available to infants, and early cognitive stimulation really does matter. Sixty years of developmental research show that parenting practices in infancy mediate links between SES and long-term cognitive outcomes (Hoff 2003; Milner 1951). Yet the hundreds of experiments in recent years exploring basic cognitive capacities at younger and younger ages have almost all focused on middle-class participants. At the 2010 International Conference on Infant Studies, less than $1 \%$ of the 1,000 research presentations reported including participants from disadvantaged families, although 20-40\% of children in the United States are growing up in poverty (Wight et al. 2010).

If the same studies conducted in all those university research centers were also run with infants in the lower-income neighborhoods that are often just a few miles from campus, the results would likely be different. We know, for example, that the development of spatial abilities presumed to be species-specific is compromised in low-SES children, who have less opportunity to exercise spatial skills than do high-SES children who have access to toys, puzzles, bikes, and the freedom to explore a safe neighborhood (Levine et al. 2005). Yet developmental textbooks abound with claims about how "infants' awareness of physical principles is evident at 3 months," or how "infants use knowledge of phonotactics to segment words by 7.5 months." Such statements may be true of the particular infants observed in the particular studies cited, but the results are often framed more broadly, as if these specific ages characterize human infants universally and differences in early experience are simply irrelevant. Would it matter if we discovered that these age-specific developmental milestones are in fact only characteristic of infants in middle-class families? If we found that infants living in poverty are actually one or several months slower than higher-SES infants to show evidence of "core knowledge of spatial relations" or "speech segmentation ability"? It should matter, because to ignore such differences is to ignore the potential role of environmental support in the ontogeny of these critical capacities.

In our longitudinal research on the early development of fluency in language understanding, we have found robust relations between verbal processing speed in infancy and long-term outcomes in both high-SES English-learning children and low-SES Spanish-learning children. In both groups, infants who are faster in speech processing at 18 months are more advanced on later cognitive and language measures (Fernald et al. 2006; Hurtado et al. 2007). But the differences in performance between these groups are stunning. By 18 months, we find that low-SES children are already substantially slower in processing speed and vocabulary growth; and by the age of 5 years, we see the gap in developmental measures found in numerous studies since the 1960s (Ramey \& Ramey 2004). This inconvenient truth has forced us to re-evaluate the assumption that our earlier research with children of affluent families licensed broad conclusions about the "speech processing abilities of 18- to 36 -month-olds" in general, given that perfectly healthy 18 - to 36 month-olds from low-income families in the neighboring community performed so differently on the same tasks.

But these findings have also led us to ask a question we had previously ignored: Could it be that differences in early experience with language contribute to the variability observed in children's efficiency in real-time processing? It turns out that early practice with language is influential in the development of fluency in understanding. In a study with low-SES families, we found that those children whose mothers talked with them more learned vocabulary more quickly - and they also made more rapid gains in processing speed (Hurtado et al. 2008). These results suggest that child-directed talk not only enables faster learning of new vocabulary - it also sharpens the processing skills used in real-time interpretation of familiar words in unfamiliar contexts, with cascading advantages for subsequent learning. By examining variability both within and between groups of children who differ in their early experience with language, we gained insight into common developmental trajectories of lexical growth in relation to increasing processing efficiency, and also discovered environmental factors that may enable some children to progress more rapidly than others.

Pinker (1994) once declared that "to a scientist interested in how complex biological systems work, differences between individuals are so boring!" In fact, many biologists these days are keenly interested in environmental influences on expression of the genetic code during early development and the resulting phenotypic differences (Gottlieb 2007; Zhang \& Meaney 2010). New research on prenatal programming shows that fast- or slowgrowth trajectories set before birth have long-term developmental consequences for health and vulnerability (Coe \& Lubach 2008). Developmental psychologists can now also address important questions about the crucial influence of early postnatal experience on cognition and language. But to do so we need to extend beyond the WEIRD "convenience samples" we have traditionally relied 
Commentary/Henrich et al.: The weirdest people in the world?

on, to examine trajectories of growth in broader populations of children living in more diverse circumstances.

\section{Cultural congruence between investigators and participants masks the unknown unknowns: Shame research as an example}

\section{doi:10.1017/S0140525X10000087}

\section{Daniel M. T. Fessler \\ Department of Anthropology and Center for Behavior, Evolution, and Culture, University of California, Los Angeles, CA 90095-1553. \\ dfessler@anthro.ucla.edu http://www.sscnet.ucla.edu/anthro/ faculty/fessler/}

Abstract: In addition to questions of the representativeness of Western, educated samples vis-à-vis the rest of humanity, the prevailing practice of studying individuals who are culturally similar to the investigator entails the problem that key features of the phenomena under investigation may often go unrecognized. This will occur when investigators implicitly rely on folk models that they share with their participants.

Henrich et al. present a compelling empirical case for a reduction in the current reliance on participants from WEIRD societies in the study of the human mind. Awareness of these facts should motivate investigators both to recognize the need for caution in inferring the generalizability of results derived from parochial samples and to pursue more diverse samples, whether directly or through collaborative networks. Here I wish to suggest that additional impetus for such changes derives not simply from questions of representativeness, but rather from the fact that investigators themselves inevitably view the world through the lens of their own culture.

As Henrich et al. observe, most psychological research is conducted by members of WEIRD societies, hence investigators are largely studying people very similar to themselves. By virtue of the fact that they, too, are enculturated humans, investigators will often share folk psychological models with participants drawn from their own cultural group. Although rigorous science is always based on the refinement of models through hypothesis testing, one has to begin somewhere, and I strongly suspect that social scientists in general, and psychologists in particular, often rely on their own folk models as a starting point in this process. When investigators share fundamental cultural commonalities with their participants, they run the risk of overlooking key features of the psychological phenomena at issue, as such features may be absent from, or downplayed by, the given folk models (see Levy 1973).

In light of the above, it is interesting to consider a domain of research only addressed in passing by Henrich et al., namely, the study of emotions. Due, in part, to the centrality of claims regarding innateness in a number of seminal modern investigations of emotion, building on Darwin's (1872) prior work on the subject, the study of emotion has long included a substantial cross-cultural component. From early on, cross-cultural research was employed to explore both the recognizability of emotional expressions (e.g., Ekman \& Friesen 1971) and the qualia and elicitors associated with different emotions (e.g., Wallbott \& Scherer 1986).

Despite this emphasis, the relative neglect by prominent universalists of the importance of cultural meaning systems in the experience of emotion eventually led to bold claims by some cultural constructionists (e.g., Lutz 1988), wherein a panhuman psychology was seen as merely the seed from which radically diverse parochial forms of emotion spring. As the field matured further, polar differences between nativists and constructivists eventually dulled, with various middle grounds being found, wherein investigators sought to systematically examine the nature and extent of cultural variation in the elicitors of emotions, the non-emotion correlates of cultural variation in emotional experience, and similar facets (see, e.g., Kitayama \& Markus 1994; Mesquita \& Frijda 1992).
While the psychology of emotion would thus seem to be one area where the call to action trumpeted by Henrich et al. has already long been heard, even here one can find signs of an underestimation of the importance of moving beyond cultural similarities between investigator and participant. For example, a burgeoning literature (a keyword search in PsycINFO produces almost 6,000 hits) explores shame. This literature focuses on self-consciousness and issues of moral and personal worth, often contrasting shame with guilt (see Tangney \& Dearing 2002). However, results obtained from a small-scale non-WEIRD society, and hints provided by many non-Western languages' emotion lexicons, suggest that, in addition to the aforementioned features, for many of the world's peoples, the emotion of shame also encompasses what English speakers would call respect and fear, facets that primarily concern subordinance in a hierarchy rather than failure to conform to social standards (Fessler 2004).

Although the subordinance aspect of shame is absent from the vast majority of the voluminous scholarly work on the subject, with guidance, English-speaking participants generally recognize this facet of shame in their own experiences. Importantly, however, they do not volunteer this association on their own. This is not surprising, given that Western folk models of shame entirely ignore the experience of subordinance, perhaps because this experience is arguably antithetical to the values common in WEIRD groups. This leads to my central question, namely, why have investigators not attended more to the subordinance facet of shame?

I suspect that the problem is not merely that the participants studied by many students of shame differ in important ways from most of the world's peoples, but also that the investigators studying those participants consistently find strong support for their own intuitions, and hence see less need to cast a wide net despite the history of cross-cultural research on emotions. Whereas early work on emotions included places such as New Guinea villages (Ekman \& Friesen 1971), and recent work on pride, directly relevant to understanding shame, has been conducted in rural Burkina Faso (Tracy \& Robins 2008), much current work on shame is confined to educated and affluent members of the state-level societies of North America, Western Europe, and, to a lesser extent, East Asia. Importantly, failure to recognize those aspects of shame that are downplayed or ignored in their own culture limits investigators' ability to explain key features of the phenomena of interest. For example, the behavioral tendencies for flight and hiding so prominent in the experience of shame seem odd in the context of a moral emotion, but are readily understood once it is recognized that this moral emotion is built upon a simpler emotion active in the dangerous context of dominance negotiations (see Fessler 2007).

The take-home lesson here is that we must be particularly cautious to avoid employing participants who are culturally similar to ourselves whenever the given enterprise involves testing predictions that resonate with our intuitions. Such samples are unlikely to reveal to us that which we do not know that we do not know, features that are masked by our own folk models.

\section{(Dis)advantages of student subjects: What is your research question?}
doi:10.1017/S0140525X10000099

\section{Simon Gächter}

School of Economics, University of Nottingham, University Park, Nottingham NG7 2RD, United Kingdom.

simon.gaechter@nottingham.ac.uk

http://www.nottingham.ac.uk/Economics/people/simon.gaechter

Abstract: I argue that the right choice of subject pool is intimately linked to the research question. At least within economics, students are often the perfect subject pool for answering some fundamental research questions. 
Student subject pools can provide an invaluable benchmark for investigating generalizability across different social groups or cultures.

In their excellent article, Henrich et al. rightly caution us to be careful when we draw general conclusions from WEIRD subject pools, of which undergraduates are the most frequently used one, also in economics. My main comment is that the right choice of subject pool is intimately linked to the research question. Since the different behavioral sciences also have different research questions, the right choice of subject pool will also often be different across disciplines. In my own discipline, economics, students are actually often the best subject pool for quite a few (fundamental) research questions. Here is why I believe so.

Economic theories normally do not come with assumptions (or even caveats) about the restricted validity to only a specific group of people; that is, they (implicitly) assume "generality." Like the assumption of selfishness, "generality" is a good assumption in the absence of rigorous data. The tools of experimental economics have been deployed to investigate the empirical relevance of the selfishness assumption (see, e.g., Fehr et al. 2002) and are now also used to probe the "generality assumption," that is, the importance of variations of behavior across population subgroups within a given society (e.g., Bellemare et al. 2008) or across societies (e.g., Herrmann et al. 2008).

However, my main point is this: The "right choice" of subject pool depends on the research question. If the researcher is interested in understanding behavioral variation between particular groups of people, then the right choice is running experiments with these people. The landmark study by Henrich et al. (2005) is a shining example. Yet, at least in economics, substantial effort is also devoted to test formal theories or to detect interesting behavioral regularities (Bardsley et al. 2010; Croson \& Gächter 2010; Smith 2010). Because economic theories normally assume generality, any subject pool is in principle informative about whether theoretical predictions or assumptions contain behavioral validity. At that stage, generalizability to other subject pools is not (yet) an issue. Among the universe of potential subject pools to test a theory, students are often the perfect one: on average, students are educated, intelligent, and used to learning. These are very valuable characteristics because, in addition to the main aspect of a theory of interest to the researcher, economic theories often assume cognitive sophistication. It therefore makes sense to control for sophistication also by choice of subject pool (in addition to clear instructions), in order to minimize chances of confounding genuine behavioral reactions to the treatment of interest with lack of understanding of the basic decision situation.

Take recent theories of social preferences (as surveyed, e.g., in Fehr \& Schmidt 2006) as an example. In addition to otherregarding preferences, these theories all assume cognitive sophistication. When testing these theories, the main point of interest is not to find out whether people are as cognitively sophisticated as the theories (maybe wrongly) assume, but to see to what extent other-regarding motives exist, holding everything else constant. Because students are typically above average with regard to cognitive sophistication, they are often a perfect subject pool for first tests of a theory. Moreover, students, unlike most other subject pools, are readily available (and cost effective). Experiments can therefore also easily be replicated, which is important to establish empirical regularity and hard to achieve with any other subject pool.

Of course, strictly speaking, observed results hold only for the subject pool from which evidence is collected. Generalizability is a generic issue in any empirical research (Falk \& Heckman 2009). However, once a clear benchmark result is established, we can proceed by testing, for example, how age and life experience matter (e.g., Sutter \& Kocher 2007b), or how results extend to more representative subject pools (e.g., Bellemare et al. 2008; Carpenter et al. 2008). Along the way, researchers often establish whether and how students differ from the general population.
As Henrich et al. point out, understanding the potential influence of cross-societal (or cultural) differences in (economic) behavior is a particularly interesting direction for investigating generalizability. But it poses further challenges, in particular if socio-demographic factors matter (as some of the above-cited research suggests). The reason is that socio-demographic influences might be confounded with genuine societal or cultural differences. The problem is exacerbated the more subject pools are actually being compared. Again, to ensure that confounds are minimized, student subject pools are often the best available choice (Bohnet et al. 2008; Herrmann et al. 2008) to establish a clean benchmark result on how people from different societal/ cultural backgrounds behave in the exact same decision situation - a fundamental question from the generality perspective of economics. The benchmark can - and should $(!)$ - then be taken as a starting point for investigating generalizability to other social groups.

\section{It's not WEIRD, it's WRONG: When Researchers Overlook uNderlying Genotypes, they will not detect universal processes}

\author{
doi:10.1017/S0140525X10000105
}

\begin{abstract}
Lowell Gaertner, ${ }^{\mathrm{a}}$ Constantine Sedikides, ${ }^{\mathrm{b}}$ Huajian Cai, ${ }^{\mathrm{c}}$ and Jonathon D. Brown ${ }^{\mathrm{d}}$

${ }^{a}$ Department of Psychology, University of Tennessee, Knoxville, TN 379960900; ' $\mathrm{b}$ Center for Research on Self and Identity, School of Psychology, University of Southampton, Southampton SO17 1BJ, United Kingdom;

${ }^{\mathrm{c}}$ Institute of Psychology, Chinese Academy of Sciences, Chaoyang District, Beijing 100101, China; ' Department of Psychology, University of Washington, Seattle, WA 98195-1525.

gaertner@utk.edu

cs2@soton.ac.uk

huajian.cai@gmail.com

jdb@uw.edu
\end{abstract}

Abstract: We dispute Henrich et al.'s analysis of cultural differences at the level of a narrow behavioral-expression for assessing a universalist argument. When Researchers Overlook uNderlying Genotypes (WRONG), they fail to detect universal processes that generate observed differences in expression. We reify this position with our own cross-cultural research on self-enhancement and self-esteem.

We dispute the level of analysis Henrich et al. have employed to conclude that members of Western, Educated, Industrialized, Rich, and Democratic (WEIRD) societies are non-representative of the human species and to determine, more generally, whether an observed cultural difference contradicts a universalist argument. Borrowing from the biological lexicon, our position is as follows: Analysis of difference at the level of a narrow phenotypic behavioral-expression precludes detection of human universals that operate at the level of an abstract genotypic process. Stated otherwise, When Researchers Overlook uNderlying Genotypes (our acronym WRONG), they will fail to detect universal processes that generate observed differences in expression (Kobayashi \& Brown 2003). We first frame our position with an example and then reify our position with our own cross-cultural research on self-enhancement and self-esteem phenomena from which Henrich et al. derived their WEIRD conclusion.

The human diet exemplifies our position (Sedikides \& Gregg 2008). When considered at the narrow level of observed behavior, human societies appear extraordinarily different in regard to what they eat (e.g., a Kosher diet precludes pork; a Jain diet is vegetarian). When considered more broadly, however, the diverse diets are connected and assimilated by a universal need for sustenance. It would be faulty indeed to conclude that the need for sustenance is less pronounced, if not absent, in one 
society because it consumes less, if not any, of the foods consumed by another society - what is consumed depends on factors such as climate and custom. Our point, of course, is that human universals operate at the abstract level of processand-function, and the expression of the universal emerges in conjunction with contextual considerations (Schlenker 1974).

To be clear, we do not oppose the study of concrete behavior. Cataloguing behavioral differences across societies certainly contributes to understanding the human condition. However, the presence of a behavioral difference per se is not evidence contrary to a universalist argument. The necessary consideration is whether the observed difference is produced by a process or function common across societies.

Henrich et al. suggest that WEIRD societies are peculiar, in part, because they uniquely possess positive self-views. Such a conclusion, however, is a consequence of the WRONG strategy. Our own cross-cultural programs of research on the self-enhancement motive (i.e., need to maintain a positive sense of self) and self-esteem (i.e., an affective self-evaluation) indicate that a positive self-view is a human tendency.

Our primary studies and meta-analytic syntheses indicate that both Westerners and Easterners self-enhance, but they do so on different attribute dimensions. ${ }^{1}$ Westerners self-enhance (i.e., deem self as superior to peers) on attributes relevant to individualism, and Easterners self-enhance on attributes relevant to collectivism. This is because Westerners deem individualism, and Easterners deem collectivism, as important. Here a common process (self-enhancing on important attributes) is differentially expressed (individualism vs. collectivism), because culture affects the expression, not the presence, of the enhancement motive (Brown \& Kobayashi 2002; Sedikides et al. 2003; Sedikides et al. 2005; 2007a; 2007b). Furthermore, that common process has the same functional association with psychological adjustment in both cultures: Self-enhancing on important attributes promotes better adjustment (e.g., greater well-being, less depression,) among Easterners and Westerners (Gaertner et al. 2008; Kobayashi \& Brown 2003; O’Mara et al. 2009). Therefore, when assessed at the abstract level of process and function, members of WEIRD societies are quite normal in their striving for a positive self-view.

As Henrich et al. suggest, Eastern samples typically provide lower explicit reports of self-esteem than do Western samples (Heine et al. 1999). Such explicit reports, however, are compromised by a pervasive modesty norm in Eastern cultures (Brown, in press; Kurman 2003). Indeed, the cultural differences occur in reports of cognitive self-evaluation, not affective self-regard, and these differences vanish when modesty is statistically controlled (Cai et al. 2007). Similarly, cultural differences in self-esteem vanish when self-esteem is assessed with implicit measures that circumvent modesty norms (e.g., Yamaguchi et al. 2007). Furthermore, self-esteem reveals the same functional patterns across cultures. Self-esteem predicts greater well-being and lower depression in the East (Cai et al. 2009), just as it does in the West (Taylor \& Brown 1988). Likewise, self-esteem bolsters against threats to self-worth in both cultures such that failure feedback more strongly erodes immediate feelings of worth for low rather than high self-esteem persons (Brown et al. 2009). Hence, when assessed at the abstract level of process and function, members of WEIRD societies are quite normal in their possession of a positive-self view.

In summary, testing human universals at the level of narrow behavioral differences between societies is the WRONG strategy. Human universals operate at the abstract level of process and function, and such universals can generate observed differences. We conclude with application of our argument to the man-to-boy insemination rituals of New Guinea with which Henrich et al. began their article. At the narrow level of the observed behavior, the rituals seem bizarre in regard to WEIRD standards. When considered more abstractly in terms of process or function (i.e., a social practice marking a boy's passage to manhood), the rituals connect with coming-of-age rituals practiced in other societies, such as the Bar and Bat Mitzvah, Credo-baptism, Debutante Ball, and Sweet Sixteen. The observed behaviors certainly differ, but the underlying psychological process is the same.

\section{NOTE}

1. Here and in the following, we use the terms Eastern and Western for expedience in reference to samples from East Asia versus samples from the United States, Canada, and Western Europe.

\section{Wired but not WEIRD: The promise of the Internet in reaching more diverse samples}

\author{
doi:10.1017/S0140525X10000300
}

\author{
Samuel D. Gosling, ${ }^{\mathrm{a}}$ Carson J. Sandy, ${ }^{\mathrm{a}}$ Oliver P. John, ${ }^{\mathrm{b}}$ and \\ Jeff Potter ${ }^{c}$ \\ ${ }^{a}$ Department of Psychology, University of Texas, Austin, TX 78712; \\ ${ }^{\mathrm{b}}$ Department of Psychology, University of California, Berkeley, CA 94720; \\ ${ }^{\mathrm{c}}$ Atof Inc., Cambridge, MA 02139. \\ samg@mail.utexas.edu www.samgosling.com \\ cjosandy@mail.utexas.edu www.carsonsandy.com \\ o_johnx5@berkeley.edu http://www.ocf.berkeley.edu/ johnlab/ \\ research@outofservice.com http://research.outofservice.com/
}

\begin{abstract}
Can the Internet reach beyond the U. S. college samples predominant in social science research? A sample of 564,502 participants completed a personality questionnaire online. We found that $19 \%$ were not from advanced economies; $20 \%$ were from nonWestern societies; $35 \%$ of the Western-society sample were not from the United States; and $66 \%$ of the U. S. sample were not in the 18-22 (college) age group.
\end{abstract}

Henrich et al. show that the vast majority of research in the behavioral sciences continues to be based on populations the authors call WEIRD because they are unlikely to be representative of humankind. Even more alarmingly, much of the research published in top-tier journals is not even representative of the populations in WEIRD (Western, Educated, Industrialized, Rich, and Democratic) countries. For example, in the 510 samples published in the Journal of Personality and Social Psychology (JPSP) in 2002, 85\% of them were student samples, $71 \%$ of the participants were female, more than $80 \%$ were white, and the mean age was 22.9 years (Gosling et al. 2004).

What should we do about this? Henrich et al. conclude their article by urging institutions to improve the infrastructure for collecting data from non-WEIRD samples as well as the incentives for studying them. However, Henrich et al. offer very little in the way of concrete practical suggestions for expanding the reach of research in the behavioral sciences.

We propose that the Internet holds great promise for broadening the participant base of research in the behavioral sciences (Gosling \& Johnson 2010; Reis \& Gosling 2010). Using the Internet, researchers can deliver to participants a broad range of graphics, photographs, and dynamic media (Krantz \& Williams 2010); obtain informant reports (Vazire 2010); and administer surveys (Tuten 2010), questionnaires (Johnson 2010), ability tests (Schroeders et al. 2010), and experiments (Reips \& Krantz 2010). Participants can be randomly assigned to experimental conditions, reaction times can be measured, and a broad range of incentives for participation can be offered (Göritz 2010).

Internet methods offer researchers many advantages over traditional methods in terms of improved efficiency, accuracy, cost effectiveness, and reach (Gosling \& Johnson 2010; Gosling et al. 2004; Reis \& Gosling 2010). But how do Internet samples fare regarding Henrich et al.'s concerns about generalizability and representativeness? We do know that Internet samples are 
generally more diverse than the "traditional" samples in the top psychology journals with respect to gender, socioeconomic status, geographic region, and age (Gosling et al. 2004). But do such samples offer any hope of reaching the kinds of participants that Henrich et al. rightly note are so under-represented in the behavioral sciences?

To find out, we examined a dataset of personality questionnaires collected in Dutch, English, German, or Spanish via the Internet in 2009 (for details of the method, see Srivastava et al. 2003). We examined only those 564,502 participants $(81 \%)$ who had indicated the country in which they resided and were aged between 9 and 90 . We followed the four contrasts used by Henrich et al.: (1) people from modern industrialized societies versus small-scale societies; (2) people from Western societies versus non-Western industrialized societies; (3) people from the United States versus other Western societies; and (4) university-educated versus nonuniversity-educated people from the United States.

With respect to the first comparison, our sample almost certainly fails to capture individuals living in small-scale societies, which is unsurprising given the written format and the languages used. So to provide some insight into the extent to which Internet samples capture participants beyond modern industrialized societies, we examined the percentage of our participants who were from the 34 countries classified by the International Monetary Fund (IMF) as "advanced economies" (WEO Groups and Aggregates Information 2009): $19 \%(\mathrm{~N}=104,928)$ were not from these advanced economies.

With respect to the second contrast, we found that $20 \%$ $(\mathrm{N}=111,962)$ of the sample were from non-Western societies (i.e., not from North America, Israel, Australia, New Zealand, and Western Europe). With respect to the third contrast, within these Western societies, 35\% ( $\mathrm{N}=158,300)$ were not from the United States.

With respect to the fourth contrast, we did not have information on whether the participants were college students. However, we do know that $66 \%(\mathrm{~N}=369,916)$ of the sample (who ranged in age between 9 and 90) were not from the 18 to 22 age group that characterizes the vast majority of college students. With respect to diversity in terms of ethnicity, a substantial $41 \%$ were non-white.

All four contrasts suggest that Internet samples are not as dominated by WEIRD participants as are samples currently published in behavioral science journals. Moreover, even though the percentages of non-WEIRD participants in the Internet samples may seem modest, Internet methods permit the collection of large samples, so the absolute sample sizes of non-Weird participants can be quite impressive. For example, although the sample was predominantly North American ( $52 \%$ from the United States and $5 \%$ from Canada), the sample represented a breadth of geographic regions from around the world: 111 countries, from Albania $(\mathrm{N}=215)$ to Venezuela $(\mathrm{N}=1,920)$, were represented in the sample by at least 100 participants each.

Our data were collected using a website that did not specifically target non-WEIRD samples and used Western languages (Dutch, English, German, Spanish) predominant in WEIRD countries; thus, the findings almost certainly underestimate the percentages of non-WEIRD participants that could be obtained in studies targeting non-WEIRD participants and using languages common in non-WEIRD samples.

Like all methods, Internet-based methods are subject to limitations. The samples are certainly not representative of humankind, participants must have access to the Internet and know how to use a Web browser, and some studies cannot be delivered online (Johnson \& Gosling 2010). However, the global spread of Web access will diminish the sampling biases. Moreover, current infrastructure and technology already provide the means for distributing compensation to participants (e.g., via Amazon.com's Mechanical Turk) and for accessing populations that do not have their own Web access (e.g., via laptop computers, tablets, and smart-phones administered by local research assistants, even those with minimal technical knowledge).

No single method can rectify the field's over-reliance on WEIRD samples. However, Internet methods are one promising tool that should be used in combination with others to combat the problem of WEIRD research. Our analyses suggest that Internet samples can help ameliorate the biases found in typical research samples and can help wean WEIRD researchers off WEIRD samples.

\section{WEIRD walking: Cross-cultural research on motor development}

\author{
doi:10.1017/S0140525X10000117
}

\author{
Lana B. Karasik, ${ }^{a}$ Karen E. Adolph, ${ }^{\text {b }}$ Catherine S. Tamis- \\ LeMonda, ${ }^{\mathrm{b}}$ and Marc H. Bornstein ${ }^{\mathrm{a}}$ \\ ${ }^{a}$ National Institute of Child Health and Human Development (NICHD), \\ Bethesda, MD 20892-7971; ${ }^{\mathrm{b}}$ Department of Psychology, New York University, \\ New York, NY 10003. \\ lana.karasik@nih.gov \\ karen.adolph@nyu.edu \\ catherine.tamis-lemonda@nyu.edu \\ Marc_H_Bornstein@nih.gov
}

Abstract: Motor development - traditionally studied in WEIRD populations - falls victim to assumptions of universality similar to other domains described by Henrich et al. However, cross-cultural research illustrates the extraordinary diversity that is normal in motor skill acquisition. Indeed, motor development provides an important domain for evaluating cultural challenges to a general behavioral science.

Henrich et al. remind us, like others before (e.g., Bornstein 1980; Graham 1992; Kennedy et al. 1984; Moghaddam 1987; Parke 2000; Russell 1984; Sexton \& Misiak 1984; Triandis 1980), about the formative role of culture in all human behavior. Even basic psychological processes such as perception are subject to cultural variation (Segall et al. 1966). Nonetheless, psychological research remains largely ethnocentric.

Consider basic processes in motor development. Cross-cultural comparisons serve as natural experiments revealing the effects of experience on motor development and highlighting diversity in developmental pathways and the range in human potential (Adolph et al. 2010; Bornstein 1995). Yet, the field suffers from long-standing assumptions of universality based on norms established with WEIRD populations.

Historically, research on motor development focused on establishing universals. Led by Gesell, early pioneers established the practice of cataloging the ages and stages of motor development. In particular, Gesell's (1928) testing procedures, test items, and developmental norms - explicitly and deliberately based on behaviors of WEIRD children - inspired the widely used Bayley (1969) and Denver Scales (Frankenburg \& Dodds 1967), which describe the developmental timing and sequence of infants' motor skills. Such normative templates are the current, accepted gold standard of motor development, and are regarded as prescriptions of what is desired, rather than relatively narrow descriptions of what may be acquired.

Due to the prevailing emphasis on motor milestones, crosscultural research has been dominated by normative comparisons of onset ages. Recent evidence shows that cultural differences in daily childrearing practices can explain accelerated and delayed onset ages relative to WEIRD norms (see Adolph et al. 2010, for a review). For example, in some regions of Africa, the Caribbean, and India, caregivers vigorously massage and exercise infants as part of daily bathing routines, stretching infants' limbs, tossing them into the air, and propping them into sitting and walking positions (Bril 1988; Super 1976). Infants who 
receive massage and exercise begin sitting and walking at earlier ages than infants who do not (Hopkins \& Westra 1988). Laboratory experiments with random assignment to exercise and control groups confirm these results: A few minutes of daily exercise accelerates walking onset (Zelazo 1983).

Reciprocally, restricted practice can delay the age at which children reach motor milestones. In Northern China, the practice of toileting infants by laying them on their backs in sandbags for most of the day delays the onset of sitting, crawling, and walking by several months (Mei 1994). Among WEIRD families, the recent practice of putting infants to sleep on their backs rather than their stomachs has resulted in delayed onset of crawling and other prone skills (Davis et al. 1998). In cultures that do not encourage crawling (including WEIRD infants circa 1900), large proportions of infants skip crawling altogether (Hopkins \& Westra 1988), either bum-shuffling or proceeding straight to walking (Fox et al. 2002; Robson 1984; Trettien 1900).

Other aspects of motor development are also influenced by culture and context. For example, childrearing practices can affect the shape of developmental trajectories. In WEIRD cultures, upright leg movements show a well-known U-shaped trajectory: Newborn stepping disappears after about 2 months of age and upright stepping does not return until the end of the first year. But in cultures where caregivers exercise infants' leg movements (and this is confirmed in laboratory experiments), stepping shows monotonic increase throughout the first year (Super 1976; Zelazo 1983).

Foot-binding in China provides an extreme example of how cultural practices affect the form of movements. For 1,000 years, mothers deformed their daughters' feet to give them the walking gait of a "tender young willow shoot in a spring breeze" (Chew 2005). Feet 3 inches in length were achieved through years of training and excruciating pain. The routine (typically beginning between 5 and 8 years of age) involved breaking four toes on each foot and bending and tightening them in place with bandages. Girls then relearned how to walk with altered balance constraints of their shortened feet. This custom was eradicated in the 1920s.

Cultural practices also affect the endpoint of development. Daily tasks require peoples of Africa, Asia, and North America to develop walking and running skills that exceed the abilities of WEIRD adults. African women and Nepalese porters of both genders carry immense loads by modifying their walking gait to conserve mechanical energy (Heglund et al. 1995). They routinely carry more than their body weight for many kilometers (Bastien et al. 2005). Tarahumaran Indian children, women, and men of Mexico run 150 to 300 kilometers round-the-clock for fun and for persistence hunting (Bennett \& Zingg 1935). Endpoints can also stop short of what is expected. Crawling on hands and feet before walking is typical in WEIRD infants, but some families of adults in rural Turkey crawl on hands and feet instead of walking (Humphrey et al. 2005). In contrast to most cultures, the parents of these adult children never encouraged walking, and the primary models for locomotion were siblings who also crawled instead of walked.

Henrich et al. raise an important point about commonalities across cultures with different childrearing practices. Basic motor functions - manual, postural, and locomotor skills - that are universally useful and adaptive are present in every society studied. We are comparing the postural and manual capacities of 5-month-olds in disparate cultures on maternal handling practices (Karasik et al. 2010). Despite different support contexts, infants practice various postures with accompanying opportunities for object exploration. These data highlight developmental equifinality (Bornstein 1995): Although the routes to object exploration vary, the outcome is the same.

Cross-cultural research on motor development is important for establishing general principles in developmental science and for revealing possibilities in human development hitherto unimagined. WEIRD infants sit at 6 months, but African infants sit at 4 months. WEIRD mothers would never dream of leaving their young infants unattended, but mothers in Cameroon leave their 5-month-olds (for 20+ minutes!) sitting alone on high stools. These sorts of phenomena can only be revealed with cross-cultural work providing the impetus for laboratory investigations to consider and test hypotheses previously not envisioned.

\section{The socio-ecological approach turns variance among populations from a liability to an asset}

\author{
doi:10.1017/S0140525X10000129
}

\section{Selin Kesebir, Shigehiro Oishi, and Barbara A. Spellman \\ Department of Psychology, University of Virginia, Charlottesville, VA 22904- 4400 . \\ sk8dm@virginia.edu http://people.virginia.edu/ sk8dm soishi@virginia.edu http://people.virginia.edu/ so5x/me2.htm spellman@virginia.edu http://people.virginia.edu/ bas $6 g$ /}

Abstract: We emphasize the value of the socio-ecological approach in addressing the problem of population variances. The socio-ecological perspective studies how social and natural habitats shape human behaviors, and are in turn shaped by those behaviors. This focus on system-level factors is particularly well-suited to studying the origins of group differences in human behavior.

The target article persuasively demonstrates the perils of building and testing psychological theories almost exclusively on Western, Educated, Industrialized, Rich, and Democratic (WEIRD) people. We wholeheartedly agree that knowledge based on WEIRD samples is limited at best and misleading at worst. The authors' plea for broadening the knowledge base of psychological science is valid, timely, and of prime importance for the future development of psychological and behavioral sciences.

Whereas the authors' main recommendation for a better science is to sample participants strategically from diverse populations, we recommend an additional research strategy based on a socio-ecological perspective. Just as ecological biologists study animals' behaviors in relation to their natural habitats (e.g., Stutchbury \& Morton 2001), socio-ecological psychologists study how natural and social habitats affect human mind and behavior. The first step we recommend is, instead of dismissing the research on WEIRD people, to consider the simple yet critical question of "Why are WEIRD people so weird to begin with?" Once potential explanations are considered, researchers can go on to systematically test these potential causes of human diversity in mind and behavior as the second step.

There already are several recent studies using a socio-ecological perspective that provide important clues as to why WEIRD people are so different from non-WEIRD people, and what factors might cause diversity in mind and behavior (see Oishi \& Graham, in press, for a review). Fincher et al. (2008), for example, wondered why nations, and indeed, regions, vary on individualism versus collectivism (with the former being typical of WEIRD countries). They showed that historically low levels of pathogen prevalence might be one reason-people in nations with low pathogen prevalence do not need to draw sharp ingroup-outgroup distinctions the way people do in nations with high pathogen prevalence. Similarly, Kitayama et al. (2006) wondered about the origins of rugged individualism in the United States, and hypothesized that it is in part due to the history of the frontier spirit. They found that people in Hokkaido, the northern island of Japan with a history of frontier spirit, showed a greater degree of American-style individualism than did mainland Japanese who don't have such a history. 
Commentary/Henrich et al.: The weirdest people in the world?

Oishi and colleagues (Oishi et al. 2007; 2009a) likewise wondered why Americans typically define themselves in terms of personality traits, skills, and abilities rather than collective attributes (Markus \& Kitayama 1991) and show conditional group identification (Cialdini et al. 1976). They demonstrated that high residential mobility might be partly responsible for such patterns of self-concepts and group identification (see Oishi, in press, for review). Uskul et al. (2008) speculated that the degree of economic interdependence might be in part responsible for analytic thinking dominant in the United States. They examined whether herders (who are economically independent) would show a greater degree of analytic tendency than farmers and fishermen (who are more dependent on others in their economic activities) in the same single region of Turkey. Indeed, they found that Turkish herders showed more analytic tendencies than did farmers and fishermen. Likewise, Yamagishi et al. (2008) hypothesized that preference for unique choice (Kim \& Markus 1999) among Americans is due in part to open as opposed to closed social systems, and they demonstrated that this was indeed the case. Finally, one of the target article authors' own seminal research (see Henrich et al. 2005) has importantly shown that market integration and payoffs to cooperation in daily economic activities predicted cross-societal variations in behavioral responses in the Ultimatum Game.

A socio-ecological perspective may help us to understand not only cross-societal variation, but variation within WEIRD populations, too. For example, mean punishment expenditures from the Public Goods Game described in the target article's Figure 4 show high diversity within WEIRD samples: The United States and Australia are at one end and Germany and Denmark are at the other end. The socio-ecological perspective helps us to generate various hypotheses regarding this variation. For instance, the United States and Australia are nations high in residential mobility, whereas Germany and Denmark are nations low in residential mobility. Could these within-WEIRD variations be the result of societal differences in residential mobility (and temporariness of group membership)? If so, would there be a comparable within-nation variation in punishment behaviors between residentially mobile cities (e.g., Atlanta) and residentially stable cities (e.g., Philadelphia), a within-city variation between residentially mobile people and stable people, and even a within-person variation between the times when people are thinking about moving and the times they are thinking about staying? In the area of self-concept and conditional group identification, Oishi and colleagues have found such within-society variations as well as cross-society variations (see Oishi, in press, for review).

We are of course not claiming that all variation between human populations is due to socio-ecological factors. There is no doubt that biological and evolutionary forces also play an important role. However, a socio-ecological perspective does provide a concrete framework for searching for the causes of diversity and universality of mind and behavior.

In conclusion, we agree that psychological knowledge should not be solely based on WEIRD people. We also agree with Henrich et al. that it is important to include large and diverse samples in our science. We recommend two additional steps for researchers. First, ask the simple, yet important question of "Why are WEIRD people so weird?" Second, test whether any potential socio-ecological factors that might make WEIRD people weird account for societal, regional, and individual variations in a broad array of phenomena central to human psychology. These two additional steps are critical because they can convert the research on limited WEIRD samples from a major liability (as the authors suggest) to a major asset from which we can build and develop the type of psychological and behavioral sciences that the authors promote in their target article - the psychological and behavioral sciences that illuminate the causes of universality and diversity in mind and behavior. Instead of dismissing the research based on WEIRD people, we can start a better science from it! We believe that a socio-ecological perspective is particularly helpful to this end.

\section{Determinants of cognitive variability}

\author{
doi:10.1017/S0140525X10000130
}

\section{Sangeet S. Khemlani, ${ }^{a}$ N. Y. Louis Lee, ${ }^{b}$ and Monica Bucciarelli ${ }^{\mathrm{C}}$ \\ ${ }^{a}$ Department of Psychology, Princeton University, Princeton, NJ 08540; ${ }^{\mathrm{b}}$ Department of Educational Psychology, The Chinese University of Hong Kong, Shatin, New Territories, Hong Kong SAR of China; ${ }^{\mathrm{c}}$ Department of Psychology, Università degli Studi di Torino, via Po, 14-10123, Torino, Italy. khemlani@princeton.edu http://www.princeton.edu/ khemlani louis@cuhk.edu.hk http://www.fed.cuhk.edu.hk/eps/people/leel.html monica@psych.unito.it http://www.psych.unito.it/csc/pers/bucciarelli/bucciarelli.html}

Abstract: Henrich et al. address how culture leads to cognitive variability and recommend that researchers be critical about the samples they investigate. However, there are other sources of variability, such as individual strategies in reasoning and the content and context on which processes operate. Because strategy and content drive variability, those factors are of primary interest, while culture is merely incidental.

Henrich et al.'s thought-provoking article discusses two major issues - how psychologists should pursue research, and how culture leads to cognitive variability. In what follows, we address these issues in turn, and argue that any cognitive theory ought to account for not only culture, but also other sources of cognitive variability.

First, Henrich et al. compare empirical data obtained from individual samples that fall under the umbrella description WEIRD (Western, Educated, Industrialised, Rich, and Democratic) with those obtained elsewhere, and draw the conclusion that "WEIRD subjects may often be the worst population from which to make generalizations" (sect. 7.1.2). For example, they report that people in many non-Western samples do not experience the Müller-Lyer illusion as strongly as do Americans, and query that "if visual perception can vary, what kind of psychological processes can we be sure will not vary?" (sect. 3.1, para. 5). However, while Henrich et al. caution against making sweeping generalizations from limited sets of data, they do not explain why they are permitted to make sweeping generalizations of relativism from their own data - the Müller-Lyer illusion is but one single phenomenon in visual perception, hardly representative of all visual perceptual processes. What would count as a representative sample of human psychology? The assertion that WEIRD participants are least representative of human psychology implies that there is a more representative sample, but Henrich et al. have failed to specify it. We believe that such a specification can only be arrived at empirically, and that it is impossible to specify a priori what a representative sample of human cognition should be.

Psychological research is theory-driven. Hence, in the absence of any evidence or theoretical rationale suggesting otherwise, WEIRD samples are a convenient proxy for conducting research, and they allow researchers to draw tentative conclusions about the matter of investigation. We acknowledge that certain specific psychological phenomena observed in WEIRD samples may not occur in other populations, and such discrepancies may help researchers make more accurate predictions in future experiments. Henrich et al.'s results underline the point that tentative conclusions are needed in order to support or contravene a theory.

Second, Henrich et al. have identified culture as a major source of cognitive variability, but we believe it is important to 
examine other sources of variability, as well. Cognitive processes are by nature non-deterministic: Children do not employ a deterministic strategy to perform cognitive tasks (e.g., Siegler 1996), and patients with dementia, head injury, ADHD, and schizophrenia are even less consistent in their thinking (for a review, see MacDonald et al. 2006). Likewise, the same individual may perform a task differently at different times. In one of our studies (Bucciarelli \& Johnson-Laird 1999), a group of participants had to draw deductions from syllogistic premises by using cut-out paper shapes representing syllogistic terms. Results showed that individuals' strategies differed from trial to trial in terms of which premises to interpret first, how to interpret the premises, and how to diligently search for counterexamples. As a result, it was impossible to predict individuals' cognitive operations based on their previous performance.

What other sorts of factors affect the way we think? Maturational and psychopathological factors are clear determinants, but the content on which a cognitive process operates may affect the process itself. Individuals think about different contents because they differ in their experiences, education, and beliefs. Culture may explain variability in these factors only to a certain extent, and hence psychologists ought to develop theories that explain how a cognitive process (a) can be modulated by content, and (b) develops and decays under normal and pathological conditions, respectively.

Consider the case of bicultural individuals. The behaviour of these individuals is guided by one internalized culture or the other at different moments (e.g., Ng \& Lai 2009; Pouliasi \& Verkuyten 2007), and they organize their cultural identities differently (Haritatos \& Benet-Martínez 2002). When bicultural individuals' cultures contain inconsistent moral values, they will experience moral dilemmas such as the following described by a 19-year-old second-generation Indian American: "I enjoy my Indian culture, I feel that it is rich in tradition, morality, and beauty; confused because I have been in many situations where I feel being both cultures is not an option ... I feel like you have to choose one or the other" (Haritatos \& Benet-Martínez 2002). How might a theory explain this phenomenon? Mental models theory (e.g., Johnson-Laird 2006) allows for individuals to entertain inconsistent beliefs, because we tend to rely on separate sets of beliefs in separate contexts (Johnson-Laird et al. 2000). This is evident in moral reasoning, in which moral intuitions and conscious moral reasoning are based on beliefs that are neither complete nor consistent (Bucciarelli et al. 2008). Our conception of culture therefore differs from that in cross-cultural psychology, which considers culture as a network of discrete, specific constructs that guide cognition only when they come to the foreground in an individual's mind (Hong et al. 2000).

How do cross-cultural differences in thinking emerge in a society? Henrich et al. explain the development of these differences by appealing to content (data perceived, norms, and connotations) and context (individuals' contemporary environment, the environment during development, and the immediate experimental environment). We emphasize that an analysis of reasoning strategies can explain variability within the same individual. Therefore, if content, context, and strategy drive cultural differences, then those factors are of primary interest, whereas cultural differences are merely incidental. Cognitive theories should distinguish between the universal processes they propose and the specific contents on which they operate. For instance, our own theory of moral reasoning (Bucciarelli et al. 2008) posits that moral reasoning is simply normal deontic reasoning (Bucciarelli \& Johnson-Laird 2005) applied to moral contents and contexts. Moral contents and contexts may differ across cultures, but the theory of deontic reasoning we propose is, and ought to be, domain-general. Such a dissociation between general computational operations and the contents they operate on allows researchers to construct theories that are sensitive not just to cultural differences, but to age-related, social, personality, and strategic differences, as well.

\section{Responsible behavioral science generalizations and applications require much more than non-WEIRD samples}

\author{
doi:10.1017/S0140525X10000142
}

Vladimir J. Konečni

Department of Psychology, University of California-San Diego, La Jolla, CA 92093-0109.

vkonecni@ucsd.edu

http://psychology.ucsd.edu/people/faculty/vkonecni.php

Abstract: There are many methodological considerations - some intricately associated with the use of WEIRD samples - that adversely affect external validity as much as, or even more than, unrepresentative sampling does. Among suspect applications, especially worrisome is the incorporation of WEIRD-based findings regarding moral reasoning and retribution into normative expectations, such as might be held by international criminal tribunals in "cognitively distant" war-torn areas.

The article by Henrich et al. is a valuable contribution that goes beyond prior critiques of the deplorable lack of representativeness of a large proportion of participant samples that have been used in the behavioral sciences. The cogency of argumentation, and both the breadth and the detail of the empirical documentation that is provided, are impressive. Therefore, my commentary will not challenge the main thesis proposed by Henrich et al. Instead, its purpose is to supplement and increase the scope of their article's argument.

An important, although perhaps self-evident, observation is that the authors' thesis concerning WEIRD samples would be even more useful (perhaps considerably more so) had they at least mentioned and briefly outlined some other factors - often closely, and sometimes unavoidably, associated with the research designs using WEIRD samples - which may even more detrimentally affect the generalizability (external validity) of the results than does the lack of WEIRD samples' representativeness.

An abbreviated list of such factors will have to suffice here: unrepresentative sets of independent variables; artificiality of research settings; a limited number of tasks (often a single task) through which the independent variables are presented; and relying on a single data-collection method (such as questionnaires, surveys, or rating scales) - and therefore obtaining a single dependent measure (or an uninformatively correlated set of measures) that is often qualitatively different from the one to which generalization is sought in the "real world." The mentioned factors are highly relevant for a more complete understanding of the issues in some of the areas discussed in the target article, especially fairness and cooperation, punishment of "excessive" cooperators, personal choice, "fundamental attribution error," and moral reasoning.

Moreover, one must worry about the (statistical) interaction of the effect of WEIRD samples' uniqueness (extremity, non-modal character) with the effects of these additional factors (e.g., the frequently highly artificial tasks), such that the overall result (especially when interactions are of a multiplicative form) would be even more misleading with regard to some real-world criterion and domain of desired application than is the case on the basis of WEIRD samples' "differentness" alone. On the other hand, if, for example, a greater variety of tasks were used, the presently observed differences between WEIRD and various nonWEIRD samples might in some cases disappear. One simply cannot predict what would happen without doing the research.

The above family of methodological observations has its root in the pioneering work of Campbell and colleagues (e.g., Campbell \& Stanley 1963; Webb et al. 1966). Among the subsequent empirical demonstrations of some of the underlying principles were the studies by Ebbesen and Konečni: for example, of decisions under risk (in automobile driving; e.g., Ebbesen et al. 1977; Konečni et al. 1976) and of key decisions by judges, prosecutors, and other participants in the criminal justice system (Konečni \& Ebbesen 1982b). An important aspect of this work has been the mustering 
of the theoretical and empirical support for the idea of validated simulations in behavioral science (Konečni \& Ebbesen 1992).

Among the judicial decisions studied in this research program were those of the setting of bail and, especially, the sentencing of felons (e.g., Ebbesen \& Konečni 1975; Konečni \& Ebbesen 1982a). This work utilized both WEIRD and non-WEIRD samples (as in the fourth "telescoping contrast" in Henrich et al.; see sect. 6) and supports the target article's skepticism. Moreover, a more general, but logical, extension is to question the applicability of WEIRD-based findings regarding aggressiveness, retribution, fairness and equity, and moral reasoning in general (cf. sect. 4.4.) to international law. Here the most troubling possibility is the deliberate or unconscious incorporation of WEIRD-based findings into the normative expectations held by international bodies in "cognitively distant" war-torn areas - such as in Rwanda by the United Nations Assistance Mission for Rwanda and the International Criminal Tribunal for Rwanda. What must be very carefully taken into account are not only the enormous complexities of ancient tribal relations, but also those stemming from massive religious conversions by some of the warring parties under an external oppressor (as in Bosnia and Herzegovina, another internationally adjudicated conflict).

In sum, there is far more to external validity than the unrepresentativeness of samples. The only truly solid reason to trust an experimental simulation (especially one that potentially involves enormous human costs) is to have had it validated by means of careful successive approximations to the real world, each step moving closer to the actual real-world phenomenon - not just with different participant samples, but also guided by a multi-method X multi-dependent-measure matrix (Konečni \& Ebbesen 1992).

Some additional observations are in order. Just as Nature Genetics requires all empirical papers to include data from two independent samples (target article, sect. 6.2, para. 3), the Journal of Personality and Social Psychology, for example, might begin to require not just the use of at least two different methods in the laboratory, but also both laboratory and field research - before researchers move away from psychology freshmen. If this were required, it seems likely that some "cute," supposedly counterintuitive, task-specific effects (including in the area of heuristics and biases) would not be replicated even with different WEIRD samples. I am not as favorably disposed as Henrich et al. apparently are to Mook's (1983) idea that the use of WEIRD samples is justified "when seeking existential proofs" (sect. 7.1.6, para. 1); nor to the authors' admittedly clever idea of setting up research facilities in bus terminals and airports to capture non-university participants (sect. 7.3, para. 6) - if the same old suspect methods, such as "reactive" questionnaires and games with trivial pay-offs, would continue to be used.

Henrich et al. believe that behavioral scientists' tendency to claim "universality" for data obtained with WEIRD participants may in part be due to so many researchers themselves being WEIRD (sect. 7.1.1, para. 8). This fact may also be partly responsible for researchers' relative reluctance to worry adequately about external validity and about the effects of complex higher-order interactions among type of participants, methods, and settings. A sustained interest in such interactions may require a contextual ("field-dependent") worldview and a holistic reasoning style that is (according to Henrich et al.) less utilized by WEIRD people, who favor analytical reasoning.

\section{When nurture becomes nature: Ethnocentrism in studies of human development}

doi:10.1017/S0140525X10000154

David F. Lancy
Program in Anthropology, Utah State University, Logan, UT 84322.
David.Lancy@usu.edu
http://www.usu.edu/anthro/davidlancyspages/index.html

Abstract: This commentary will extend the territory claimed in the target article by identifying several other areas in the social sciences where findings from the WEIRD population have been over-generalized. An argument is made that the root problem is the ethnocentrism of scholars, textbook authors, and social commentators, which leads them to take their own cultural values as the norm.

I am grateful to the authors of the target article for illuminating this very serious problem in the social and behavioral sciences. I also have written critically on the issue, but without the courage to fully assert the fundamental weirdness of researchers' favorite subjects. I will add to Henrich et al.'s catalog by briefly reviewing several areas where the WEIRD tribe can be shown to be extreme outliers.

Culture and cognitive development. The best known model of cognitive development originated with the Swiss biologist, Jean Piaget. He derived his theory largely on his observations and interactions with his own very brainy and sophisticated children (Vidal 1994). As Piaget (and colleagues) tested his propositions, subjects were largely drawn from the same milieu of middleclass European society. Piaget led the vanguard but a veritable army of cognitivists followed in his wake. The models that emerged were rooted entirely in research with children from the WEIRD tribe. Had these scholars delved into the anthropological literature, particularly with respect to the cognitive processes implicated in native belief systems, they might have paused to consider the implications. Indeed, Alexander Luria, close colleague of Lev Vygostsky, traveled to Central Asia in the 1930s and easily discovered alternative patterns of thinking in the reasoning of Uzbek peasants (Luria 1976).

Later, researchers working in West Africa (Dasen et al. 1978; Greenfield 1966) and Papua New Guinea (PNG) (Kelly 1971) sought to test these theoretical ideas about children's cognitive development outside the West and found that they didn't hold up very well, especially beyond early childhood. As Luria had earlier shown, scholars were finding that cognitive "development" was driven by exposure to modern institutions - schooling, in particular - rather than reliably erupting, like second molars (Cole et al. 1971). Others succeeded in showing very specific connections between cultural practices and cognitive skill (Price-Williams et al. 1969). Somewhat later in PNG, the typical two-culture (WEIRD vs. "other") comparison was broadened to systematically assess cognition in a variety of societies with varying subsistence patterns and degrees of acculturation (Lancy 1983). These studies revealed that the patterns of cognitive behavior in the WEIRD population were uncommon compared to preferred local alternatives (Lancy \& Strathern 1981).

Culture and children's social behavior. Social psychologist Millard Madsen began with the premise that Western middleclass children were markedly different. He devised a series of ingenious, game-like devices that unambiguously revealed whether a child was disposed towards a competitive or cooperative stance. In his initial work, he found that subjects in the United States made only competitive moves in the game (which only rewarded cooperative moves), whereas children from a Mexican village made only cooperative moves. Replicated in numerous other societies, the studies revealed U.S. children as outliers, being much more competitive than children from other societies (Madsen 1971). Further cross-cultural variation was neatly predicted by the child's social circumstances, so village kids were found to be more cooperative than urban kids, for example. In the highlands of PNG, Melpa children from warring clans were less cooperative than pairs from the same or allied clans (Lancy \& Madsen 1981).

Culture and parent-child interaction. The problem identified by Henrich et al. arises, I believe, from a (likely universal) ethnocentrism. Contemporary orthodoxy regarding child development and child-rearing can turn nurture into nature. The way WEIRD parents raise their children becomes more than just the current fashion, it becomes "natural," rooted in the phylogeny or history of the species. This can be quickly illustrated.

Working among the Gusii of Kenya, LeVine (2004) has raised doubts about widely accepted tenets of the theory of infant attachment. Like many, if not the majority of mothers throughout 
history, Gusii mothers respond to their infant's need for sustenance but otherwise largely ignore them. Such behavior, if displayed by a Euro-American mother, would be grounds for a clinician to predict later pathological development. Of course, the Gusii children turn out fine. Closely related is the practice of talking to nonverbal infants using a special speech register (baby talk or motherese). Often assumed to be both universal and essential to the development of speech in children, it is in fact neither (Ochs \& Schieffelin 1984). "Parenting style" theory (Baumrind 1971) cannot withstand cross-cultural scrutiny. Central African Bofi farmers fit the so-called authoritarian parenting style in valuing respect and obedience and exercising coercive control over their children. Bofi children should, therefore, be withdrawn, non-empathetic, and aggressive, and lack initiative. On the contrary, they display precisely the opposite traits, leading Fouts (2005) to conclude that the theory "has very little explanatory power among the Bofi” (p. 361).

Parent-child play is another in this basket of parenting behaviors that illustrates how nurture is made out to be nature. A recent textbook describes variation in patterns of parent-child play, but never questions its universality (see Scarlett et al. 2005). One scholar of infancy claimed that the absence of mother-infant play signaled attachment failure or worse (Trevarthen 1983, p. 151). Empirical studies of mother-child play typically report that "Mothers were instructed to play with their [2- to 3-years-old] children as they would at home" (Stipek 1995, p. 244; emphasis added). Another common feature is the use of well-off, highly educated subjects, with no caveat about the limited generalizability of the results (see Sung \& Hsu 2009, p. 432). However, in a recent review of the ethnographic record, with hundreds of cases, parent-child play was found to be extremely rare and distinctly incompatible with many native ideas about "best practices" (Lancy 2007). To parents, play's chief value is in keeping children out of the way (Whittemore 1989, p. 92).

Lastly, I would demur from the notion that parents' active teaching of children is both universal and the essential component of cultural transmission (Csibra \& Gergely 2009; Strauss \& Ziv 2004). A thorough survey of ethnographic and historical cases shows teaching by parents to be extremely limited (Lancy \& Grove 2010); children are expected to learn from observation, imitation, and practice. As Fiske (1997) notes, in the ethnographic record there is "much less child-rearing than there is culture-seeking” (p. 11).

\section{BIZARRE chimpanzees do not represent "the chimpanzee"}

\section{doi:10.1017/S0140525X10000166}

David A. Leavens, ${ }^{a}$ Kim A. Bard, ${ }^{b}$ and William D. Hopkins ${ }^{c}$

a School of Psychology, University of Sussex, Falmer, East Sussex BN1 9QH, United Kingdom; ${ }^{\mathrm{b}}$ Psychology Department, Centre for the Study of Emotion, University of Portsmouth, Portsmouth, Hampshire PO1 2DY, United Kingdom; ${ }^{\circ}$ Division of Psychobiology, Yerkes National Primate Research Center, Atlanta, GA 30322, and Department of Psychology, Agnes Scott College, Decatur, GA 30030.

davidl@sussex.ac.uk

http://www.sussex.ac.uk/psychology/profile114996.html

Kim.Bard@ port.ac.uk

http://www.port.ac.uk/departments/academic/psychology/staff/

title,50477,en.html

whopkins@agnesscott.edu

http://userwww.service.emory.edu/ whopkin/

Abstract: Henrich et al. convincingly caution against the overgeneralization of findings from particular human populations, but fail to apply their own compelling reasoning to our nearest living relatives, the great apes. Here we argue that rearing history is every bit as important for understanding cognition in other species as it is in humans.
Henrich et al. provide a timely cross-cultural analysis of perceptual and cognitive abilities in humans, concluding that a significant number of allegedly human-universal capacities are, in fact, confined to specific socio-cultural environments. Ironically, they caution against overgeneralizing from particular humans to all humans, everywhere, but fail to correctly generalize their own arguments to nonhuman species. In section 7.1.4, Heinrich et al. highlight both the need for and the limits of comparisons between human and nonhuman primates. Their point is that contradictory conclusions about the taxonomic distribution of cognitive abilities could be reached, depending upon which human cultures are sampled. What they fail to elucidate is that precisely the same arguments also apply to nonhuman primates, particularly the great apes, humans' nearest living relatives. The over-reliance in psychology on one group of humans, WEIRD, to represent "the human" in cognitive terms has a strong parallel in the over-reliance in comparative psychology on chimpanzees raised in Barren, Institutional, Zoo, And other Rare Rearing Environments (BIZARRE) to represent "the chimpanzee." Cross-species comparisons are appropriate only with careful consideration of specific rearing environments (e.g., Bard 1998; 2008; Bard \& Leavens 2009; Boesch 2007; Bulloch et al. 2008; Leavens 2002; 2004; 2006; Leavens \& Racine 2009; Leavens et al. 2005a, 2008; 2009; Lyn et al. 2010; Racine et al. 2008).

The case of manual pointing provides an example of overgeneralization in both humans and nonhumans. Western scientists portrayed pointing with the index finger as a human species-specific gesture adapted for referential communication (Butterworth 2003; Eibl-Eibesfeldt 1989; Povinelli \& Davis 1994; Povinelli et al. 2003). Degos (2001) recently asserted that “[ $t]$ he pointing gesture is exclusively human, and universal within mankind" (p. 263). In fact, although widespread, index-finger pointing is neither universal nor necessarily the predominant manner of pointing even where it is found (Enfield 2001; Wilkins 2003). The formerly popular idea that pointing with a particular finger extended reflects the shared evolutionary history of all humans, everywhere, exemplifies the kind of reckless disregard for cultural variability that Henrich et al. rightly criticize. Production and comprehension of pointing are widely viewed as indices to cognitive processes supporting reference, perspective-taking, and the construction of shared goals (e.g., Tomasello 2006).

Like humans, chimpanzees display group differences in pointing. Figure 1 depicts three pointing phenotypes, all emanating from the same gene pool (Leavens 2004; Leavens et al. 2005a; 2008). As this figure makes clear, depending upon which chimpanzees are sampled, entirely contradictory conclusions can be reached as to whether pointing is a shared characteristic of great apes and humans. Consider the incidence of manual pointing: If home-reared or language-trained apes were compared with humans from cultures that prohibit manual pointing, one could reach the erroneous conclusion that great apes point manually, whereas humans do not; this would be a consequence of overgeneralizing from both of the specific human and chimpanzee populations sampled. Recently, numerous researchers have reached the erroneous conclusion that only humans point declaratively, because they have compared people of largely European descent (i.e., WEIRD) with wild chimpanzees or institutionalized (i.e., BIZARRE) chimpanzees. When chimpanzees are raised the way human children are raised, in richly interactive contexts, the chimpanzees will go on to point - point with the index finger, frequently point declaratively, and comprehend pointing by others (Itakura \& Tanaka 1998; Leavens et al. 2005a; 2008; Lyn et al. 2010).

Different populations of chimpanzees also display different communicative expressions and calls. For example, Figure 2 depicts Merv pointing with his index finger to a squeeze bottle filled with juice (out of camera view). The protruded lower lip is glossed as the "juice-me" face, and to our knowledge appears only in chimpanzees that experience juice delivery from a bottle with a downward-pointing spout. Chimpanzees adapt calls to serve functions in captive environments that differ from 


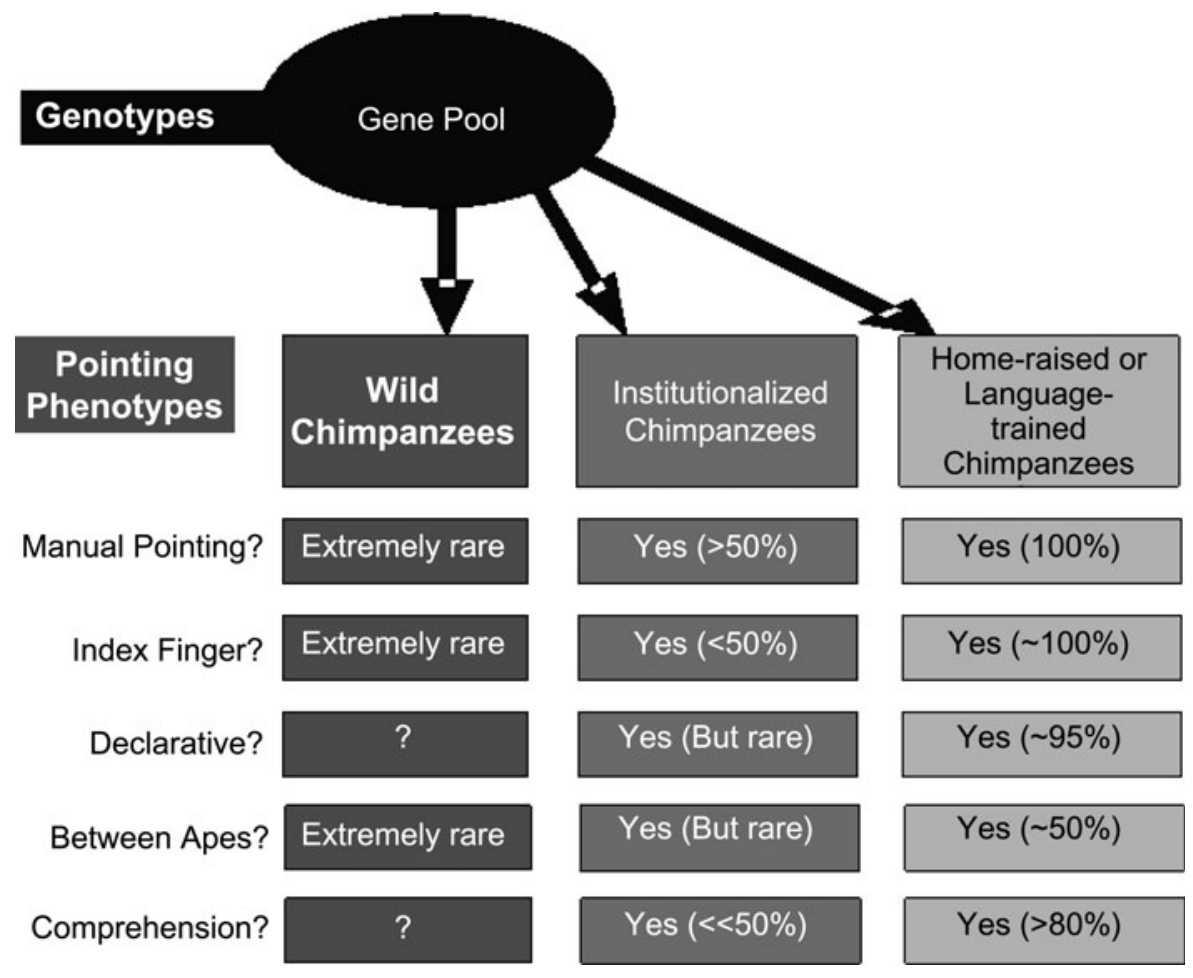

Figure 1 (Leavens et al.). Pointing incidence, posture, function, and social use differ radically between different populations of chimpanzees. Manual pointing: Extension of arm and at least one finger in triadic contexts. Index finger: Pointing with outstretched arm and index finger. Declarative: Pointing to draw somebody's attention to an object or event; includes responses to queries, such as pointing to an object when asked where that object is. Between apes: Pointing by one chimpanzee for another chimpanzee. Comprehension: Understanding pointing by others. [Sources: Pointing in wild chimpanzees: Inoue-Nakamura and Matsuzawa (1997); also see Pika and Mitani (2006). Pointing in institutionalized chimpanzees: de Waal (1982); Leavens and Hopkins (1998); Leavens et al. (1996; 2004a; 2005b); Savage-Rumbaugh (1986); among others. Pointing in home-reared or language-trained chimpanzees is nearly ubiquitous and has been described by Carpenter et al. (1995); Fouts et al. (1982), Kellogg and Kellogg (1933), Krause and Fouts (1997), Lyn et al. (2010), and Witmer (1909), among others. See Leavens and Racine (2009) and Leavens et al. (2009) for reviews.]

their uses in the wild: chimpanzees in captivity selectively emit attention-getting calls and other auditory signals, tactically, as a function of whether an observer is looking at them (Hopkins et al. 2007; Hostetter et al. 2001; Leavens et al. 2004b; 2010).

The validity of Henrich et al.'s argument does not suddenly collapse when applied to other species. We agree with Henrich

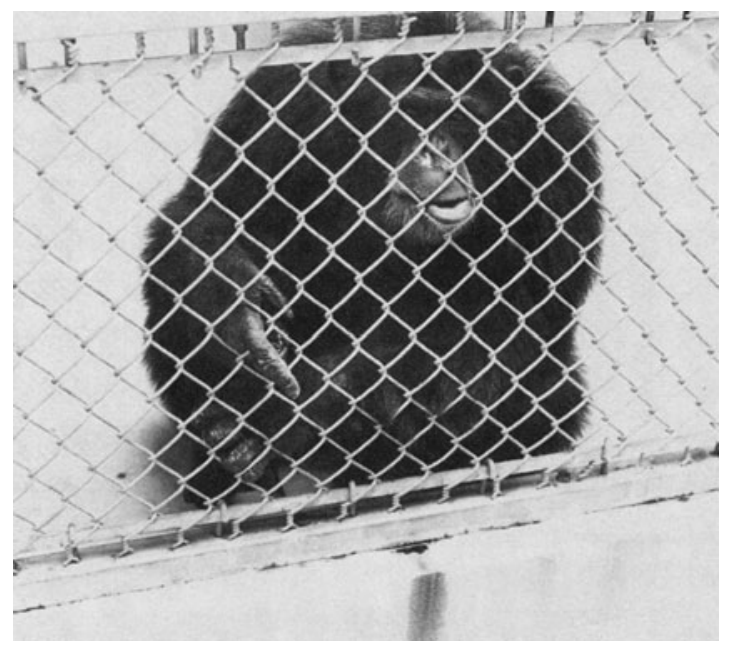

Figure 2 (Leavens et al.). Merv points with his index finger and simultaneously displays the "juice-me" face, a facial expression unique to certain captive groups. Used with permission from the American Psychological Association (Leavens \& Hopkins 1998). and his colleagues that scientists need to specify to "which people" their findings can be generalized (sect. 7.3). And we argue here, that, given the variability in communicative signaling characteristics in different populations of great apes, there is no rational justification for overgeneralizing from BIZARRE chimpanzees to the entire chimpanzee species. Like humans, chimpanzees evince different cognitive adaptations to different environments; no single environmental context can elicit the full range of chimpanzees' cognitive capacities.

\section{ACKNOWLEDGMENTS}

We gratefully acknowledge discussions about these ideas with Sarah Boysen, the late George Butterworth, Josep Call, Malinda Carpenter, Deborah Custance, Mark Krause, Heidi Lyn, Simone Pika, Timothy Racine, and Jamie Russell.

\section{Explaining why experimental behavior varies across cultures: A missing step in "The weirdest people in the world?"}

doi:10.1017/S0140525X10000178

\section{Edouard Machery}

Department of History and Philosophy of Science, University of Pittsburgh, Pittsburgh, PA 15260

machery@pitt.edu

www.pitt.edu/ machery/

Abstract: In this commentary, I argue that to properly assess the significance of the cross-cultural findings reviewed by Henrich et al., 
one needs to understand better the causes of the variation in performance in experimental tasks across cultures.

Henrich et al. review a large body of evidence showing that in numerous tasks Westerners (often Americans) behave differently from people in other cultures. The target article raises numerous important questions, including: Which psychological characteristics should we expect to vary from culture to culture (studying these would necessarily be cross-cultural) and which should we expect to be universal (studying these could be done on the basis of samples of convenience)? Why are most examples in Henrich et al.'s article drawn from social psychology? Is social cognition particularly likely to vary from culture to culture? Would we find the same cross-cultural variation if we focused on the phenomena discussed in perceptual and cognitive psychology textbooks (provided the cross-cultural data are available)? Do the findings reviewed by Henrich et al. suggest replacing the traditional search for human psychological universals (our human nature) with the search for laws that connect psychological variation to social and ecological conditions?

Although these questions are fascinating, for the sake of space I will focus only on the following issue: It is unfortunate that Henrich and colleagues chose to say little about the causes of the cross-cultural variation they present evidence about, since the significance of this variation depends on the exact nature of these causes. To illustrate this point, I examine three possible explanations of the cross-cultural variation described by Henrich et al., and I consider how these explanations bear on the significance of the findings presented in the target article.

It is well known that when participants conceptualize a given experimental task differently (perhaps because they have different beliefs about the experimenter's goals), they behave differently. (In fact, this is a well-known experimental problem.) To give a single example, Westerners behave differently in economic games depending on how these are framed (e.g., Hoffman et al. 1994). Some of the findings discussed by Henrich et al. (perhaps many) may simply be due to the fact that experimental participants in different cultures conceptualize the experimental tasks differently. In fact, Henrich's own findings (in previous work with other colleagues: Henrich et al. 2004; 2005) about the cross-cultural variation in the Ultimatum, Dictator, and Public Goods games can be at least partly explained in this way, as some researchers on Henrich's team reported that participants assimilated the economic games to real-life situations (e.g., the Orma identified the Public Goods Game with the harambee, a local financial contribution to collective projects; see also Cronk 2007).

Naturally, when participants in an experimental task (within a given culture or across cultures) behave differently because they conceptualize this task differently, this variation does not show that the individuals who behave differently differ psychologically (i.e., that they have different psychological mechanisms, capacities, characteristics, etc.): If one controlled for the differences in task conceptualization across cultures, the crosscultural variation would disappear. Thus, much of Henrich et al.'s thunder would be stolen if the findings they describe were due to people in different cultures conceptualizing the tasks differently. True, it would still be incorrect to expect people in different cultures to behave similarly in a given experimental task, but this would not entail that the American mind differs from, for example, the Peruvian mind or the Machiguenga mind.

Consider now a second type of explanation. Human beings have evolved numerous domain-specific mechanisms that are designed to interact with the cultural, social, and ecological environment to produce typically (but not necessarily) locally adaptive psychological phenotypes. Although there is little space here to discuss the various ways of specifying this hypothesis (for detail, see Fessler \& Machery, forthcoming), let us consider some possibilities. Some evolved mechanisms might have parameters which are set to different values in different environments during development. If the hypothesis of a universal grammar developed by Chomsky is correct, this is how natural languages work. Some evolved mechanisms might provide a template that is completed with culturally local information (see, e.g., Barrett's [2005] hypothesis of a universal, evolved mechanism for learning concepts of dangerous animals). It might also be that humans typically possess various strategies for fulfilling a given psychological function (e.g., categorizing or making decisions under uncertainty) and that they learn to rely preferentially on the strategies that are most efficient in their environment, while being able to revert to the other strategies if needed or primed. Many cross-cultural findings might result from people learning to rely preferentially on a particular strategy among the toolbox of strategies that are available to them, since, as discussed by Henrich et al., it is sometimes easy to prime people to adopt the cognitive styles of cultures they do not belong to. If the cross-cultural variation in experimental tasks described in the target article were due to the interaction of universal processes and local environments, this variation would reflect a genuine psychological variation a significant conclusion: It would show that across cultures, people do harbor different psychological processes (characteristics, styles, etc.), or, at least, that they preferentially rely on different processes (styles, etc.). However, this type of explanation would undermine the idea, suggested throughout Henrich et al.' article, that the Western mind is really peculiar, since the psychological differences across cultures would emerge from the same basic psychological endowment. In a sense, the cross-cultural variation in psychological phenotype would be shallow (particularly if it is merely a matter of people relying preferentially on different strategies in different cultures). Furthermore, albeit being an incomplete research strategy, in need of complementary cross-cultural work, studying Western participants could cast some light on this basic endowment, exactly as one can learn about the universal grammar by studying English syntax.

Consider, finally, a third explanation. Participants in different cultures behave differently in experimental tasks because people acquire different psychological processes, traits, or capacities across cultures as a result of cultural transmission, domaingeneral learning mechanisms, and the like. To give a single example, this third explanation plausibly applies to the cross-cultural variation in semantic intuitions (for review, see Machery, forthcoming). Philosophers of language have ignored the possibility that the semantic intuitions on which theories of reference are based might vary across cultures. However, evidence shows that while Americans tend to view the reference of proper names as determined by the causal and historical connections between these names and particular individuals, Chinese are more likely to view the reference of proper names as determined by the information speakers associate with these names (Deutsch et al. 2010; Machery et al. 2004). Follow-up studies even suggest that Americans are much more likely to hold the former kind of intuitions than are other Westerners such as French participants (Machery \& Stich, forthcoming; Machery et al. 2009). If this third explanation explained not only the variation in semantic, epistemological, and other intuitions described by experimental philosophers (see Stich's commentary), but also the findings summarized by Henrich et al., these findings would then reveal not only that the human psychological phenotype varies across cultures, but also that this variation does not merely result from the interaction of a basic psychological endowment and local environments. Furthermore, studying American participants, as most American psychologists have done for about a century, would often reveal nothing about universal properties of the mind; rather, American psychology would often just be the psychology of Americans. 


\section{WEIRD languages have misled us, too}

\section{doi:10.1017/S0140525X1000018X}

\section{Asifa Majid and Stephen C. Levinson \\ Max Planck Institute for Psycholinguistics, Nijmegen 6500AH, The Netherlands. \\ asifa.majid@mpi.nl http://www.mpi.nl/people/majid-asifa stephen.levinson@mpi.nl \\ http://www.mpi.nl/people/levinson-stephen}

Abstract: The linguistic and cognitive sciences have severely underestimated the degree of linguistic diversity in the world. Part of the reason for this is that we have projected assumptions based on English and familiar languages onto the rest. We focus on some distortions this has introduced, especially in the study of semantics.

This commentary rounds out the picture convincingly advanced in the target paper by considering how scientific approaches to language have also ignored the diversity of behavior in the linguistic domain. In the BBS paper "The Myth of Language Universals," Evans and Levinson (2009) argue that there is little evidence for the view that the variation in language structure is tightly bounded by linguistic universals. Instead, what we find is extensive variation on almost every dimension, with the main patterns understandable in terms of cultural evolution.

Why does it matter to psychology that languages vary fundamentally on so many dimensions? Leaving aside that it is the highest learned human skill, and therefore has an interest in its own right, there are at least two further reasons: (a) Language is in many ways a "window on the mind," and (b) semantic variation seems to correlate with psychological variation on a range of parameters. As a result, most of our ideas about how humans reason or what notions form natural categories are prompted by our own languages.

We must leave to the historians of science an explanation for how the myth of language universals came to dominate the language sciences for 50 years. But one factor is almost certainly the view that familiar languages such as English are canonical. Yet many features of English are quite unusual - for example, only $1.6 \%$ of languages express Yes/No questions by word order inversion (Dryer 2008), and no other known language has verb inflection with non-zero exponent for third person (as in John come-s) but zero for all other persons (see rara\#34 http://typo.uni-konstanz.de/ rara/). As we discuss here, there are languages without such "natural" concepts as left, in, arm, or green. English is, in fact, in a special, rather unusual corner of the design space for human languages, an outlier, not some kind of central default model at all.

As surveyed in the target article (sect. 3.4), there appears to be significant variation in the spatial frames of reference employed crossculturally, largely predictable from language - that is, languages without spatial left and right terms are used by peoples who prefer allocentric coding. Some authors find these results hard to credit and have wondered whether they don't reflect conscious ratiocination correcting an innate egocentric bias (Li \& Gleitman 2002). Haun and Rapold (2009) now produce additional evidence for the depth of allocentric coding in a non-WEIRD culture, Hai\|om hunter-gatherers of Namibia. Haun and Rapold asked 8year-olds to learn a demonstrated dance, and then got them to repeat the dance under 180-degrees rotation. The Hai\|om kids maintained the dance moves in absolute (North-South-EastWest) coordinates under rotation, whereas a matched German sample maintained the dance moves in egocentric coordinates (in terms of left/right motions). Given the rigidly egocentric nature of neural coding for body position, these findings are quite unexpected and show the extent to which a different spatial framework can be adopted in the cognitive representation of spatial movement.

The spatial encoding of topological relations between objects in non-WEIRD languages also challenges long-held assumptions about the human mind. Developmental psychologists and linguists have supposed that preverbal infants have a stock of prelinguistic concepts (either inborn or learned through sensorimotor experience) and that language maps onto these universal primitives (e.g., Clark 1973; Slobin 1973). These include notions such as in, on, and under - exactly those concepts found in English. Infants less than a year old show sophisticated understandings of such relations (Baillargeon 1994). But subsequent crosslinguistic investigation has shown considerable variation in how languages express spatial concepts (Bowerman 1996; Bowerman \& Choi 2001). Korean speakers distinguish between "tight-fitting" and "loose-fitting relations" instead of "containment" (as in in) or "support" (as in on). This finding has had a profound impact on how we currently think about the infant mind. Rather than the infant coming to the world with only English spatial categories, she comes now with Korean ones, too (Hespos \& Spelke 2002; McDonough et al. 2003), and researchers are actively pursuing which of the myriad further distinctions coded in other languages may be present for the infant, too.

In another domain, vision scientists have been impressed by the correspondence between the algorithms used by the visual system to parse the world around us and their reflection in language. Hoffman and Richards (1984, p. 82), after discussing how parts are assigned when viewing a face, conclude that: "It is probably no accident that the parts defined by minima are often easily assigned verbal labels" - the caveat being "in English." The parts assigned verbal labels in other languages differ substantially. In Jahai, a language spoken in the Malay Peninsula by a group of nomadic hunter-gatherers, speakers make fine-grained segmentations of the body and face: there is no term corresponding to face, arm, or leg (Burenhult 2006). Compare this with Lavukaleve, spoken by some thousand-odd subsistence fishers and farmers on the Solomon Islands. Lavukaleve speakers have a much coarser-grained system and use a single term to refer to arms and legs, with no finer-level conventionalized terms for the limbs (Terrill 2006). The correspondence between English part categories and those identified by the vision sciences has caused researchers to seriously misjudge the issues involved in a theory of the language-perception interface (Majid 2010).

Take color as yet another domain. Here language plays a critical role. Languages carve up the spectrum into a number of discrete categories, and it is these linguistic categories that are utilized in memory and perception (Davidoff et al. 1999; Gilbert et al. 2006). Speakers of languages from WEIRD societies make finer divisions in the color space than do speakers of most of the thousands of other languages of the world (Kay \& Maffi 2008), and the pinnacle of color categorization (in terms of how many divisions of the color space a language makes) has been taken to be 11 - exactly the number that English has (Berlin \& Kay 1969; Kay \& Maffi 2008). But there is accumulating evidence that WEIRD societies may be surpassed in this domain. In Russian (Corbett \& Davies 1995), Turkish (Özgen \& Davies 1998), Greek (Thierry et al. 2009), and Japanese (Uchikawa \& Boynton 1987), there are 12 terms (an additional distinction is made between dark and light blue). The new pinnacle is 15, as demonstrated by Korean (see Roberson et al. 2008).

Human performance diversity offers a rich resource for cognitive scientists. It allows us to triangulate on underlying properties of mind that would be invisible if we were all culturally identical. Instead of lamenting the loss of the "psychic unity of mankind," we should embrace the study of cognitive diversity as a window on human cognitive potential.

\section{WEIRD societies may be more compatible with human nature}

doi:10.1017/S0140525X10000191

\section{Alexandra Maryanski}

Department of Sociology, University of California-Riverside, Riverside, CA 92521.

alexandra.maryanski@ucr.edu

Abstract: Are WEIRD societies unrepresentative of humanity? According to Henrich et al., they are not useful for generalizing about 
humans because they are at the extreme end of the distribution for societal formations. In their vision, it is best to stick with the "tried and true" traditional societies for speculations about human nature. This commentary offers a more realistic starting point, and, oddly enough, concludes that WEIRD populations may be more compatible with humans' evolved nature than are most traditional societies.

While Henrich et al. exaggerate the extent to which undergraduates are used to generalize about pan-human traits (as many social scientists still refuse to subscribe to the premise that a human nature exists), it is true that such generalizations are often implicit in research findings. I also endorse the authors' call for more cross-species research and new research designs. But I strongly disagree with the claim that WEIRD populations skew the traits that characterize Homo sapiens. Henrich et al. also overstate the value of the cross-cultural method for uncovering the evolutionary foundations of human cognition and social behavior. Let me highlight some of these limitations.

What is to be learned from cross-cultural research? The crosscultural method has a long history It originated in the 19th century when ethnographers took to the field to "discover" the fundamental and universal core of humans and society. These ethnographies, along with the Human Relations Area Files (HRAF), are readily available in most libraries. What researchers found is that cultures are organized around systems of common meanings that members use to direct behaviors and organize social relations. A key finding is that despite a remarkable diversity, broad-based cultural universals do exist, and therefore, the cross-cultural method has proven to be useful in making generalizations about human cognitions, personality, culture, and society. But it has failed to uncover an anchor of underlying pan-human traits.

Today, these populations speak only in the "ethnographic present." Hunter-gatherer societies, who monopolized 95\% of human history, are extinct. Most horticultural societies are also extinct or hardly recognizable from their past history, as they have had to adapt to a 21st-century industrialized world. The cross-cultural method is still valuable, of course, but no matter what samples of societies are used, claims about pan-human psychology and social behavior are not easily made from the data. We still confront the problems of earlier researchers: How do we distinguish the simplest, oldest, and most exotic traits from the most common traits? For, once humans abandoned the hunter-gatherer lifestyle, institutional arrangements and secondary adaptations were set into motion and passed down from generation to generation, including symbolic traditions unique to a population. Hence, even if we believe that a substratum of evolved human proclivities exists, they are always at the mercy of culture and social forces that can repress them, channel them towards more normative behaviors, or, in some societies, allow them to be expressed. How, then, do we identify an evolutionary-derived trait from a socio-cultural trait? Hence, on what empirical grounds can the authors claim that WEIRD people are not in tune with their evolved nature? What yardstick justifies such a claim? In the future, would it really be a surprise that as populations around the globe slowly industrialize and set up democratic institutions, they too may come to resemble WEIRD populations? Who, then, should we nominate as unrepresentative of our species? The authors' call for new research designs for uncovering evolved traits, however, is much more promising.

A call for new research programs. Do human proclivities exist that characterize our species? Rather than falling into assumptions about human nature, we do need new research designs that include an evolutionary perspective, cross-species research, and a true interdisciplinary approach. In particular, I think we need to start by searching our evolutionary past for clues. Let me illustrate with two basic questions on human biology and human sociality: (1) How much genetic variability exists in the human genome; and (2) are humans naturally social?
Low genetic diversity. Recent findings reveal that the human genome has less genetic diversity than the chimpanzee (Pan), our closest living relative (Bakewell et al. 2007; Gagneux \& Varki 2001; Kaessmann \& Paabo, 2002: Kaessmann et al. 1999). This surprisingly low level of genetic variability suggests that humans today are all descendants of a small "mother" deme that may have numbered only in the hundreds (Long \& Kittles 2003; Relethford 2002). If so, despite great human cultural variation, a high probability exists for a bedrock of evolved traits. In addition, the fossil record tells us that anatomically modern humans evolved about 150 thousand years ago (Garrigan \& Hammer 2006; Smith et al. 2007). This means that hunter-gatherer populations were around for at least 140,000 years before horticultural farmers. As data on studied huntergatherers clearly demonstrate, they share a remarkable number of institutional arrangements with traits that include high individualism, reciprocity, and low levels of inequality; and their wellpreserved ethnographies are surely a more fruitful resource for clues into our evolved nature.

How naturally social are humans? Humans are evolved apes This fact has important implications for how we might go about studying our biologically based propensities and social arrangements. One way to gain insights into the distant past is to use cladistic analysis, social network analysis, and evolutionary theory. One intriguing finding is that monkeys (which make up $70 \%$ of all primates) are high-density collectivists, whereas great apes are low-density and low-sociality individualists, with needs for autonomy and mobility. Witness the orangutan, which is nearly solitary. Thus, one starting point for clues into our evolved nature is to study our primate relatives along with past huntergatherer societies. These data suggest that the high-sociality, collectivist touchstone we have used to characterize Homo sapiens may be misguided. And oddly enough, since the days of hunter-gathering, the society that best fits this view of human nature - at least in terms of placing a high value on individualism, mobility in space, relative autonomy, verification of self, sexual equality, and freedom of choice - are WEIRD populations. For, despite all the multiple ills of industrialized societies, WEIRD societies may be more compatible with our human nature than the high-density kinship constraints of horticultural societies or the "peasant" constraints of agrarian societies with their privileged few (for data on this argument, see Maryanski \& Turner 1992; Turner \& Maryanski 2005; 2008).

\section{It's not just the subjects - there are too many WEIRD researchers}

\section{doi:10.1017/S0140525X10000208}

\section{Michael Meadon and David Spurrett \\ School of Philosophy and Ethics, University of KwaZulu-Natal, Howard College Campus, Durban 4041, South Africa. \\ michael.meadon@gmail.com \\ http://ionian-enchantment.blogspot.com \\ spurrett@ukzn.ac.za http://ukzn.academia.edu/DavidSpurrett}

Abstract: A literature in which most data are outliers is flawed, and the target article sounds a timely alarm call for the behavioural sciences. It also suggests remedies. We mostly concur, except for arguing that the importance of the fact that the researchers themselves are mostly outliers has been underplayed. Improving matters requires nonWestern researchers, as well as research subjects.

Henrich et al. provide an important piece of "consciousness raising": They remind us that human beings are complicated and diverse, and review evidence that the variance in some types of behaviour and cognitive performance is far greater than has often been assumed. These facts have been obscured by the WEIRD-ness of the vast majority of the research subjects 
in behavioural science: people are not interchangeable in the same sense in which a sample of gold, whatever its origin, can be taken to the laboratory and treated as representative of gold generally. No one geographical, cultural, or socio-economic subgroup of people (least of all, perhaps, contemporary Western undergraduates) is representative of humanity. This means theories tested only or largely against a narrow range of subjects are likely to be incomplete or outright false. Behavioural scientists, in other words, have engaged in precipitate theorizing: outliers are unlikely to constrain explanatory theories correctly or inspire the right sort of hypothesizing.

To remedy this bias, ideally, we need globally representative samples of subjects in order to generate thorough descriptions of cognitive phenomena, guide hypothesis formation, and rigorously to test theories. The logistical and financial challenges of conducting research on this basis are immense. Overcoming them requires, among other things, changes in the research culture and to academic incentives (here Henrich et al.'s recommendations are spot on). In psychology, for example, the current incentives produce many small, single-institution, lowpowered studies aimed at discovering novel effects. What we need, however, is not, say, evidence of yet more ways of modulating implicit associations in American undergraduates. Rather, we need large, prospectively designed, highly powered, cross-cultural studies that can answer specific questions more definitively. Medical research provides a model behavioural researchers would do well to emulate. In general and in outline, before some clinical intervention is approved for use, research must proceed through three stages. This begins with small openlabel studies in Phase I; proceeds to larger, single-blind trials in Phase II; and culminates in large, multi-center, randomized, double-blind, placebo-controlled trials in Phase III. Behavioural scientists conduct too many studies in the equivalent of Phases I or II, with too few large, definitive and cross-cultural studies. There is still room for small studies - important novel phenomena undoubtedly await discovery. We merely suggest more "Phase III" research. Pooling resources, exploiting various online collaboration tools (e.g., Nielsen 2008), and shifting editorial policies and research priorities should result in more large, cross-cultural studies being conducted.

That noted, Henrich et al. underplay - to the point of missing - that how the behavioural sciences research community itself is constituted introduces biases. That the subject-pool of behavioural science is so shallow is indeed a serious problem, but so is the fact that the majority of behavioural researchers are themselves deeply WEIRD. People in Western countries have, on average, a remarkably homogenous set of values compared to the full range of worldwide variability (Inglehart \& Welzel 2005), and the data Henrich et al. adduce suggest similarly population-level homogeneity in cognitive styles. Moreover, academics are more uniform than the populations from which they are drawn (as the target article's Contrast 4 suggests), so it is not implausible to think behavioural scientists are even WEIRD-er than their most common subjects. Henrich et al. review a body of studies and experiments that did not strike those who designed and conducted them as focused on outliers. Intelligent scientists acting in good faith conducted, peerreviewed, and published this research, honestly believing in many cases that it threw light on human nature. This forcefully illustrates the power of the biases on the part of researchers themselves. It also suggests that, besides widening the pool of subjects, there are significant gains to be made by broadening the range of inputs to the scientific process, including in the conception, design, and evaluation of empirical and theoretical work. Given that diverse groups are demonstrably better at some kinds of problem solving (e.g., Hong \& Page 2004; Lakhani et al. 2006), as things stand, the WEIRD-dominated literature is robbed of potentially worthwhile perspectives, critiques, and hypotheses that a truly global research community could provide. Clearly, simply increasing the number of behavioural sciences researchers will, in general, be beneficial. Our key contention, though, is that the marginal benefits of additional Western researchers are much smaller than the marginal benefits of more non-Western researchers, among other things, just because they are non-Western.

The non-Western world, in short, can contribute not only additional subjects to experiment upon - the main focus of the target article's recommendations - but also additional researchers, with novel perspectives and ideas and who are less affected by WEIRD biases. (Naturally, these researchers will have biases of their own. Our claim is not that there is someone who consistently knows better than WEIRD researchers. It is that diverse groups of investigators can avoid some kinds of error.) Clearly, these researchers will have to be educated, will likely be middle class, and, since science flourishes in politically open societies, they will tend be concentrated in liberal countries. Nevertheless, additional non-Western researchers, even if they are educated and relatively wealthy, could be a boon to the behavioural sciences.

A direct and powerful way to remedy both sources of bias too many WEIRD subjects and too few non-WEIRD researchers - is to foster research capacity in the non-Western world. Non-WEIRD researchers tend to study non-WEIRD subjects, so increasing their number will deepen the subject pool and widen the range of inputs to the scientific process at the same time. Building research capacity, however, should not merely involve collaborations led by WEIRD researchers; it should aim to generate studies led and initiated by non-Western researchers. Committed and long-term inter-institutional collaboration between Western and non-Western universities focused on remedying the deficits in the behavioural sciences literature should include internships at Western universities for nonWestern researchers, stints at non-Western universities for WEIRD researchers, and extensive student exchange programmes (especially for graduate students). Unlike many existing scholarship and exchange programmes in the sciences, a key point of the necessary programmes should be for the learning to proceed in both directions.

\section{Development: Evolutionary ecology's midwife}

doi:10.1017/S0140525X1000021X

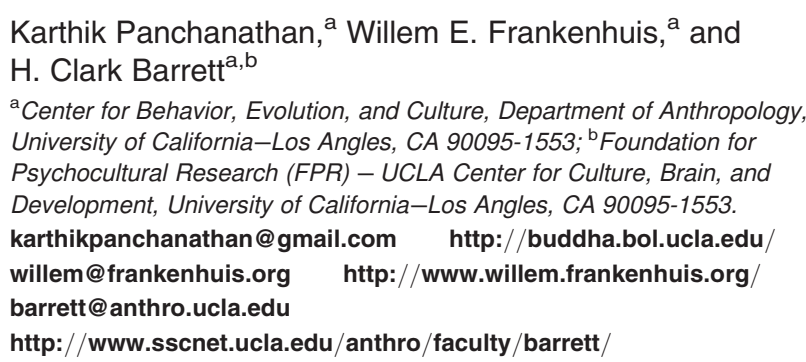

Abstract: We agree with Henrich et al. that documenting cultural universality and variability provides an indispensable window into human nature. We want to stress the mediating role development plays between evolution and culture. Moving beyond the mere documentation of universality or variability, developmental approaches can provide mechanistic explanations, linking ecology to phenotype. Combining phylogeny and adaptationism, evolutionary approaches can explain the properties of developmental systems.

The target article epitomizes a growing appetite for interdisciplinary research, bridging balkanized fields such as psychology, economics, anthropology, and biology. This integration requires "a research program that can explain the manifest patterns of similarity and variation by clarifying the underlying evolutionary and development processes" (sect. 7.2, para. 2). Development 
must be a foundation stone upon which this new program is built - if not, to stretch our titular metaphor, the conceived integration will be stillborn.

Confronted with a catalogue of human universals (e.g., social exchange) and cultural variation (e.g., rituals), social scientists traditionally link universals with evolutionary processes and variation with developmental ones. Echoing Henrich et al., we believe this is a false choice. Among other reasons, developmental mechanisms, including the learning abilities giving rise to cultural transmission, are products of natural selection; universality can arise for several reasons, natural selection being one of them (Jablonka \& Lamb 2005); and, evolutionary processes can result in adaptive plasticity, developmental systems capable of constructing a range of adaptive phenotypes, contingent on the local ecology.

To illustrate how natural selection can tailor development to local conditions, let us consider the soapberry bug, a half-inchlong, seed-eating insect dwelling in the Southeastern United States (Carroll 1993; Carroll \& Corneli 1995). While it takes a male soapberry bug only ten minutes to copulate with a female, he may spend hours more anchored to her by means of specially designed genital hooks. This mate guarding increases fitness by preventing rival males from copulating with a female before she lays her eggs. However, guarding comes at a price - males could be copulating with additional females. The local sex ratio arbitrates this opportunity cost: fewer females leads to more mate guarding.

In Oklahoma, where sex ratios vary between populations, males exhibit adaptive plasticity, calibrating the amount of mate guarding to the sex ratio experienced during development. By contrast, in Florida, where sex ratios don't vary, male soapberry bugs engage in a fixed amount of mate guarding, and, when raised in lab conditions with variable sex ratios, are incapable of calibrating. Soapberry bugs teach us why natural selection and development should not be seen as opposites. Natural selection designs developmental mechanisms, and these mechanisms give birth to phenotypes adapted to their local ecologies. When environments routinely vary, natural selection can engineer developmental mechanisms that use experience to facultatively adjust behavior.

Some of the variation across human cultures may be due to calibration, analogous to mate guarding in soapberry bugs. For example, women growing up in harsh environments - where life-expectancies are lower - exhibit earlier onset of menarche and younger age of first birth than women growing up in safe environments (Ellis et al. 1999). The explanation for this accelerated reproductive strategy, seen in other animals as well, may be a quantity/quality trade-off: When long life is a given, organisms invest in prolonged growth and development, resulting in fewer but higher quality offspring; when life is short, organisms forgo further growth and development, focusing instead on maximizing the number of offspring (Belsky et al. 1991). The developmental system, in this case determining the timing of reproduction, can thereby produce a correlation between ecology and behavior. Variation in cultural practices, such as coming of age rituals, may then partially reflect the interaction between evolved developmental processes and the state of the environment.

Of course, humans are more complex than soapberry bugs. In addition to calibration, human developmental systems can be "open," enabling the acquisition of novel skills and information. When combined with culture, a repository of wisdom accumulated across generations, novel skills and information can be passed directly to other individuals, bypassing genetic transmission (Richerson \& Boyd 2005). Infants and children are thus tasked with extracting adaptive cultural information in order to become competent adults; while adults are tasked with teaching them. The lesson, here, is not that social learning precludes evolutionary explanation; instead, the psychological mechanisms subserving cultural transmission should be viewed as adaptations (e.g., Csibra \& Gergely 2009).
However, culture is more than a consequence of social learning adaptations; culture can impose selection pressures on developmental processes, altering their genetic compositions (i.e., gene-culture coevolution; Richerson \& Boyd 2005). As Henrich et al. discuss, the ability of some people to consume milk into adulthood, particularly those from European and some African populations, provides a clear-cut example: A cultural adaptation, pastoralism, and its consequence, the prevalence of milk, created a novel selection pressure on genes, prolonging the production of lactase, an enzyme needed to digest milk sugars. Research on how coevolutionary processes shaped human cognition and development is still in its infancy.

Although Henrich et al. survey the rich breadth of the human experience, it is worth underscoring just how special we are: No other animal occupies as many different ecologies, no other animal deploys a comparable range of subsistence techniques, and no other animal exhibits as wide a range of social structures. The propensity for this plasticity makes sense only in the light of evolution. Fear not: An evolutionary explanation need not be simplistic; a thorough explanation of human plasticity requires, at a minimum, phylogenetic, paleo-ecological, cross-cultural, and adaptationist considerations. For example, recent research on past climates points not to a static evolutionary ecology, but to one in which climate change was the norm (reviewed in Richerson et al. 2001). Further, the timescale of this climatic variation was short, particularly during the late Pleistocene $(120,000$ to 10,000 years ago) when environments changed radically on the order of hundreds to thousands of years, a situation best tracked by cultural adaptation, rather than genetic evolution (too slow) or individual learning (too error prone). Our human nature, housing a rich array of evolved developmental mechanisms capable of open-ended, facultative adaptation, may have been conceived in this nurturing cradle of change.

Learning precisely how the human mind emerged from the evolutionary process poses a challenge that some believe insurmountable (e.g., Lewontin 1998). We remain optimistic. Progress will be made as research becomes increasingly interdisciplinary. Scholars interested in developmental processes will benefit from attending to cross-cultural studies, as processes often reveal themselves through their manifestations in different ecological contexts. An understanding of developmental processes will benefit students of culture, as development links ecology to behavior. An evolutionary perspective can illuminate why humans have the particular developmental mechanisms they do, given our species' evolutionary history.

\section{ODD (observation- and description-deprived) psychological research}

doi:10.1017/S0140525X10000221

Tage S. Rai ${ }^{\mathrm{a}}$ and Alan Fiske ${ }^{\mathrm{b}}$

a Department of Psychology, University of California-Los Angeles, Los Angeles, CA 90095; ' Department of Anthropology, University of CaliforniaLos Angeles, Los Angeles, CA 90095.

trai1@ucla.edu raitriumphant@gmail.com

afiske@ucla.edu www.rmt.ucla.edu

Abstract: Most psychological research consists of experiments that put people in artificial situations that elicit unnatural behavior whose ecological validity is unknown. Without knowing the psychocultural meaning of experimental situations, we cannot interpret the responses of WEIRD people, let alone people in other cultures. Psychology, like other sciences, needs to be solidly rooted in naturalistic observation and description of people around the world. Theory should be inductively developed and tested against real-world behavior.

We applaud Henrich et al. for their cogent demonstration of the need for more representative samples in psychological research 
in order to permit generalization to the human species. However, even if participant samples are representative, the psychology elicited by experiments that require participants to make judgments in response to hypothetical situations, answer abstract questions, or behave in response to artificial laboratory tasks may not be representative of - or even very informative about - human psychology across most domains of life. To understand human nature, our methods must explore the psychology of natural human experience. By this, we mean that psychological theory must be grounded in detailed observation and description of everyday life across cultures in order to understand the cognitive mechanisms that operate in the naturally occurring situations to which humans are adapted.

Observation- and description-deprived (ODD) research programs often wander far from real-life psychology because they become increasingly oriented to addressing the precedents and frameworks of previous ODD research and theory. For example, Henrich et al. show how theories about judgments of fairness and cooperation have to take into account the culture of the people making those judgments. But there is a further problem with basing our psychological theory on studies of economic games: Behavior in artificial games does not correlate strongly to social behavior in the community (Gurven \& Winking 2008). If the cooperative behavior and fairness judgments we want to understand are those that occur in everyday behavior in communities, rather than only those that are specific to the particular artificial framework of the Ultimatum Game, then we need real-world validity. That is, we need experiments that make sense to participants because the psychology of the experiment matches the psychology of behavior in the real world. ODD psychological research programs rarely provide evidence regarding the ecological validity of the results based on experiments that typically use extremely impoverished stimuli, severely restrict responses, or are based on hypothetical scenarios and Likert-scale responses to questions about abstract concepts.

Moreover, we can only interpret data if we know how participants have interpreted the research situation, the task, and the stimuli. For example, WEIRD people are used to identifying themselves and stating their interests and values, and typically welcome the opportunity to do so. But even WEIRD Scandinavians find this an uncomfortably unnatural practice; on the first day of seminar, a Scandinavian student whose turn comes to "say a bit about yourself" is embarrassed and confused by this American practice, which feels uncomfortably self-promoting (Lotte Thomsen, personal communication). In Burkina Faso, Moose informants find any personal questions threatening and demeaning: "To ask about my thoughts, desires, or activities is to seek control over me, possibly in order to thwart or harm me." Given this interpretation of an interview or questionnaire, Moose responses mean something quite different from the responses of Americans, who tend to perceive personal questions as a welcome invitation to assert themselves and make themselves look good.

If we do not know the psychocultural meaning of an experience, we cannot understand the meaning of responses to the experience. Attachment research is based on infants' behavior when their caretakers leave them in a "strange situation." In most African cultures, infants are on the body of their mother or other close kin much of the time, sleep with them, and are never out of sight of their immediate family members. Families expect children to form inalienable bonds of interdependence with them. In contrast, German infants sleep alone, are often left in daycare for many hours with strangers, and in early childhood are expected to play alone and are often left at home unattended (LeVine \& Norman 2001). German parents foster self-reliance and autonomy. Consequently, being separated from the mother in the strange situation has completely different meanings for African and German infants, so their responses cannot be directly compared.
Psychological theory over the past 40 years has been formulated mostly on the basis of prior theory, data, and intuitions. As researchers are largely from WEIRD populations, the theoretical constructs that inform experimental design tend to be based on WEIRD intuitions and stimulated by ODD data and theory. If our goal is to understand human thinking and behavior in the world, we must leave our desks and begin collecting an extensive and rich body of naturalistic descriptive data based on various kinds of observation. Currently, experimental papers are accorded the highest prestige in psychology and comprise the vast majority of studies published in top journals (Cialdini 2009). Experimental controls are invaluable, but they are useless if the constructs being tested are invalid or the experiment elicits unnatural psychology. A natural science of psychology should be based on naturalistic study in the real world of diverse situations in diverse cultures. Just as botany, zoology, ecology, geology, astronomy, chemistry, and physics grew out of, constantly return to, and must ultimately be validated by observations of the natural world, so psychology should be. In addition to relying on analytic and functional approaches, psychological constructs should be cultivated inductively from observation and we should grow our theories by contemplating naturally occurring patterns of action. Experiments are one way to test such constructs and theories, but they are not the only way. Often they are insufficient if we want to make inferences about behavior outside the lab, beyond key presses and pencil marks.

\section{What is really wrong with a priori claims of universality? Sampling, validity, process level, and the irresistible drive to reduce}

doi:10.1017/S0140525X10000233

\author{
Philippe Rochat \\ Department of Psychology, Emory University, Atlanta, GA 30322. \\ psypr@emory.edu \\ http://www.psychology.emory.edu/cognition/rochat/lab/index.html
}

Abstract: Catchy acronyms such as "WEIRD population" are good mnemonics. However, they carry the danger of distracting us from deeper issues: how to sample populations, the validity of measuring instruments, the levels of processing involved. These need to be considered when assessing claims of universality regarding how the mind works "in general" - a dominant and highly rewarded drive in the behavioral and brain sciences.

As behavioral scientists, we have to come to terms with the fact that, if we want to survive in our culture, publishing and building a reputation that brings recognition, not to mention salary and grant money, it is better to account for what is stable and predictable. It often has better academic pay-off than trying to account for what is diverse and variable.

In academia, a priori claims of universality sell better than diversity, which complicates rather than simplifies matters. Universality claims get more attention because they are cleaner and sharper, projecting more encompassing control and predictive power in the field. Such claims are also better didactic tools. They have all-around greater impact and appeal. This tends to relegate diversity to noise rather than a primary object of study.

There is no clear escape from such reality. Yet, as scientists, we have to be constantly reminded of how bully-ish and presumptuous we intrinsically are in our reductions and generalizations. This is what Henrich et al. do pointedly in their target article. They deserve to be congratulated for their effort, in the footsteps of Arnett (2008) and Rozin (2009). However, there are some important points that they either omit, or at least seem to gloss over too quickly. 
First of all, the serious issue of universal validity cannot be resolved by just testing a larger, more culturally diverse population. The sampling of diversity is crucial. The idea of setting up labs at international airport terminals, as recommended in the article, will simply not do it. Think about it: Who is traveling to foreign countries by plane? Those internationally "rich" ones would arguably be no more diverse (hence representative) than college students. They might be as "weird" and non-representative, not a good measure of what is universal or variable about the mind. As a measure of the sampling issue problem, be reminded that $80 \%$ of the world's population lives on a family income of less than $\$ 6,000$ a year, with half of the world's population living on an average of 2 dollars a day (Kent \& Haub 2005; UNDP 2006). Who would claim that poverty has no impact on shaping the mind? However, dividing the world into rich and poor would include major confounds, and the question is where to set the limits of the population category boundaries?

The question, somehow eluded in Heinrich et al., is: how to sample diversity and what rationale to adopt? What are the criteria? Geographic? Ecological? Physiological? Socioeconomic? Demographic? Cultural? These are important considerations and, in the end, it all depends on the theory behind the quest for universality. Diversity cannot be controlled just for the sake of it, particularly if the ultimate motive is to determine what is invariant and what changes across populations. The delicate question, somehow glossed over by the authors, is: On what basis do we cluster populations? Such decision can often be "shaky." For example, catchy acronyms like "WEIRD" for a population sample are good mnemonics. However, they carry the danger of distracting us from deeper issues. The last letter, D, for example, stands for "Democratic." What does this mean, given that many Eastern cultures would not consider themselves as non-democratic, having universally elected parliaments in their countries? In using such an acronym to characterize a population sample, the authors must have a theory about what democrats and a democracy mean. They must also have some intuition as to what kind of impact such a regime might have on its citizens, as opposed to another. The democratic criterion would deserve more articulated rationale. It is not as easily measurable compared to race, wealth, or education, yet it probably captures something important. The question is what?

The name of the game in science is to generalize from limited population samples: samples that are easily accessible, easy to work with, and closer to the culture we as scientists know best. In this respect, there is nothing wrong about ethnocentrism. From such sampling we keep building paradigms on how "children," "folks," and "the mind" work. In the scientific context, the claim of universality is neither wrong nor presumptuous. It is a goal and a measure of scientific truth. The problem is that such a measure is always relative to its instrument.

What seems to be really wrong with a priori claims of universality is not the lack of population diversity, per se, as implied in Heinrich et al. It is the use of Western "WEIRD" instruments to measure behavioral phenomena across cultures. The use of Westernized procedures and experimental paradigms thought out to fit Western intellectual ecology and preoccupations (e.g., two-dimensional symbolic optical illusions such as the MüllerLyer one, instead of ecologically more valid, hence potentially more universal, optic phenomena such as the "moon illusion"; the use of "weird" anonymous, and on the whole abstract, oneshot economic games; "weird" abstract questionnaires and other I.Q. testing) might well account for why typical Western samples are systematic outliers displaying extreme, non-representative behaviors. Behavioral sciences, historically a WEIRD practice, create culturally specific objects and tools of investigation to measure our predominantly WEIRD population against other populations. This is the true guilty aspect of ethnocentrism.

Last but not least, it is essential to distinguish levels at which universality claims may apply. The macro levels of economic games or self-assessment surveys are incommensurably more subject to population variability compared to lower level processes such as face recognition or emotional expression. This needs to be taken into consideration, the problem being not only how different populations are, but instead and more importantly, how populations vary in their distribution. But this distribution certainly depends on whether we are dealing with a low-level processing, such as emotion recognition, or higher level, such as self-concept.

To conclude, it would be disingenuous to think that we are not in the business of trying to approximate natural laws, whether these laws account for the homogeneity or diversity (context and culture dependence) of phenomena that, by consensus, need to be falsifiable. This is the context in which scientific claims of universality should be read and discussed. Whether population variability is ignored or not, the assumption of universality is true until proven wrong. As in any scientific debate, there is no end in sight.

\section{The weirdest people in the world are a harbinger of the future of the world}

\author{
doi:10.1017/S0140525X10000312
}

\section{Paul Rozin}

Department of Psychology, University of Pennsylvania, Philadelphia, PA 19104-6241.

rozin@psych.upenn.edu

Abstract: Although North American undergraduates represent about $0.2 \%$ of humanity, and a very unrepresentative subset, they actually provide an advance look at what humanity is becoming. In the face of globalization, this is all the more reason to study the wonderful variants of the human condition before they become homogenized.

I am enthusiastically positive about Henrich et al.'s target article. The message is central. Although the bottom line has been mentioned before in print by some of the authors and others (including myself), never has it been so thoroughly documented and elaborated into all the domains in which it is relevant. And never so convincingly.

For studies of humans in their social world, the North American undergraduate (NAU) does not serve as the fruit fly or E. coli has served for genetics. But at the level of basic psychological processes, such as learning, motor organization, or vision, the NAU is probably a pretty good fruit fly. Many basic psychological processes seem about the same in almost all humans, and great progress made by psychologists in areas such as vision can be attributed in large part to the appropriateness of NAU subjects, as well as animal models, of course. However, in the social world, in understanding humans as whole creatures negotiating the world, the NAU is a very unfortunate choice. Convenience, of course, is the justification, and this is very important; fruit flies and $E$. coli are very convenient ways to study genetics. North Americans constitute an atypical $5 \%$ of humanity, and North American freshmen and sophomores at research universities constitute an even more atypical 0.1 or $0.2 \%$ of humanity. But even for North Americans, the freshman or sophomore is very atypical, because this person is at a unique life transition, between family life and an entirely peer-centered life usually away from direct family influence. I have shown that on a number of beliefs about the social world, the grandparents of NAUs are as much like Asian Indian college students as they are like their American grandchildren (Rozin 2003).

A sample of 30-year-old Americans would give us a much better picture of American adulthood, because such individuals usually have a reasonably settled life course and family. It is ironic that the one special value of undergraduates for psychology 
has to do with how humans, in one important cultural setting, manage the transition to independence. Psychologists, at various points in time, have been interested in transition periods such as weaning, toilet training, entry into the school system, and adolescence. Here is another interesting transition point that receives surprisingly little study, for a population that is highly appropriate to examine human adaptation to change.

But the main point of my commentary is that although the NAU is truly anomalous, this subspecies of Homo sapiens is a vision of the future. With the Internet, ready availability of information of all sorts, computer fluency as key to success in the world, and ease in negotiating a world where text as opposed to face-to-face interactions are the meat of human relationships, the NAU is at the vanguard of what humans are going to be like. So study of them has some justification, not for understanding the human condition now or in history, but as to what it may become more like in the future. This is, as it were, a lucky break for academic psychology, a consequence of globalization and the computer/media revolution. Already, there has been a major change in an important area of socialization. It is now parents who are learning from their children how to navigate the Internet, order online, and navigate the modern electronic world. This is a real change in the direction of knowledge transmission. Insofar as the social world has transmuted to email, Facebook, and ordering online, it is the undergraduates who best illustrate how humans will function. Globalization, the growing availability of access to computers and the Internet, Internet dating services, the decline of face-to-face markets, automated telephone answering services, WalMart, and the like, are rapidly homogenizing the world, making more and more people like NAUs.

Because of globalization, it is especially important that we understand now the different worlds that humans have created - the physical worlds (e.g., cities, markets, architecture), the institutional worlds, the social alliances, and the mental maps of the world - before they become much more homogenized. So the prescient virtues of the NAUs make it more important that we fully explore human potential and human history at this time. We don't have much time, and the distinctive and elaborated different cultural worlds of interpersonal interactions, institutions, value systems, and the like, are a threatened species. The wide range of cultures in the world are wonderful and enlightening examples of the human condition and human potential, and we should cherish them and rush to understand them.

\section{Donald Campbell's doubt: Cultural difference or failure of communication?}

\section{doi:10.1017/S0140525X10000245}

\section{Richard A. Shweder}

Department of Comparative Human Development, University of Chicago, Chicago, IL 60637.

\section{rshd@uchicago.edu}

http://humdev.uchicago.edu/people/faculty/shweder.shtm

Abstract: The objection, rightfully noted but then dismissed by Henrich et al., that the observed variation across populations "may be due to various methodological artifacts that arise from translating experiments across contexts" is a theoretically profound and potentially constructive criticism. It parallels Donald Campbell's concern that many cultural differences reported by psychologists "come from failures of communication misreported as differences." Ironically, Campbell's doubt is a good foundation for investigations in cultural psychology.

Near the end of the last essay ever written by the great psychologist Donald T. Campbell, one reads:
I would like to close ... with a focus on the most ubiquitous source of error in efforts to know the other. This is to interpret as a cultural difference what is in reality a failure of communication. ... I personally am convinced that many of the cultural differences reported by psychologists and others using questionnaires or tests come from failures of communication misreported as differences. (Campbell 1996, p. 165)

I hope the present target article by Henrich et al. has an impact on the way research is designed and reported in psychology: that there will be more specification of the boundary conditions for generalizations about human behavior, a greater reluctance to rush to claims about fundamental processes, and far less reliance on collecting data from college undergraduates. Here I focus on Don Campbell's doubt. I suggest that the objection, rightfully noted but then dismissed by Henrich et al., that "the observed variation across populations may be due to various methodological artifacts that arise from translating experiments across contexts" (sect. 7.2, para. 2) is a theoretically profound and potentially constructive criticism.

Evaluating claims to universality of psychological theories in the light of cross- cultural evidence is of course not a new enterprise. An early example is Margaret Mead's (1932) critique of Jean Piaget's claims in his book The Child's Conception of the World (Piaget 1929). Piaget described young Swiss children as animistic and proposed a universal ontogenetic sequence in which animism waned over time. Mead produced evidence from a small-scale society in the Admiralty Islands suggesting that among the Manus, the young children were dreary literalists and only learned to be animists later in life. Henrich et al. write: "As children already show large population-level differences, it is not obvious that developmental work can substitute for research across diverse human populations" (sect. 3.1, para. 5). Margaret Mead would not be surprised.

An even earlier example is the 1895 Cambridge University Torres Straits expedition headed by the anthropologist A. C. Haddon, which set up an experimental psychological laboratory in New Guinea (see Cole 1996). William McDougall ran experimental tests of sensation concerning the limen of dual impression upon the skin, using a small pair of carpenter's dividers with blunt metal points. Native subjects were asked to perform an apparently straightforward task following an apparently easy-totranslate instruction: to say "one" or "two" as they judged whether one or two points touched the skin. McDougall reported that "Murray Islanders have a threshold of tactile discrimination of which the value, in terms of distance of two points touched, is just about one half that of Englishmen, or we may say in other words, that their power of tactile discrimination is about double that of Englishmen" (quoted in Titchener 1916, p. 206).

The claim was challenged by E. B. Titchener, whose 1916 essay defending the proposition that "human nature is much the same the world over" should be read by every anthropologist and cultural psychologist who believes otherwise (and I am one of them; see Shweder 1990; 1991; 2003). Titchener raised a series of methodological objections, including the way subjects interpreted the purpose of the task. He argued that the subjects in New Guinea, when deciding whether to report "one" or "two," were looking for "a sensation perceptively different from that yielded by a single point," which is not exactly the same as looking for two distinct points of sensation. Educated Englishmen, Titchener suggested, simply interpreted the notion of duality of impression in a stricter sense. He judged that the evidence from New Guinea did not show that the Murray Islanders' powers of tactile discrimination were substantially different from those of subjects in his own WEIRD (Western, Educated, Industrial, Rich, and Democratic) society.

The relevant methodological point can be expressed this way: Evidence of psychological differences between cultural groups may simply (although significantly) demonstrate that different stimulus situations produce different responses. The identity of a stimulus situation (e.g., an experimental task) does not exist 
independent of the subject's interpretation of its meaning. Hence, the more different the culture studied from the culture where the stimulus situation was invented, the more likely the meanings of the task will be harder to communicate with the requisite exactitude in distant field contexts.

A revealing illustration can be found in cross-cultural research on the Ultimatum Game (see Henrich et al. 2005). Were crosscultural researchers working across 15 cultures in fact able to standardize the practice and subjective meaning of "playing a game with an anonymous other"? Consider, for example, the behavior of the Au and Gnau peoples of Papua New Guinea. Many "proposers" offer more than $50 \%$ of the available currency. Many of these offers are turned down by the "responder," leaving both "players" with nothing. The "WEIRD" populations of the world don't play that (apparently strange) way.

What description of goals, values, and pictures of the world can help us understand what the stimulus situation actually meant to those New Guinea subjects? Henrich et al. (2005) offer an explanation: "The rejection of seemingly generous offers, of more than half, may have a parallel in the culture of status-seeking through gift-giving found in Au and Gnau villages, and throughout Melanesia. In these societies, accepting gifts, even unsolicited ones, implies a strong obligation to reciprocate at some future time" (p. 811). Is that not prima facie evidence that the very idea of playing with an anonymous other did not compute or translate well in the mind or language of those non-WEIRD subjects, and that in effect they were not playing the same game as the one played by a typical "weird" American college student?

Indeed, the very idea of cultural difference might well be described as not playing the same game. Hence, we learn much about the culture-specific mentality of Melanesian peoples by trying to give a "thick description" of their local goals, values, and pictures of the world, so as to understand how and why the Ultimatum Game becomes a different stimulus situation (a different affordance, if you prefer that concept) as it crosses borders and travels around the world. I suspect Don Campbell would have been pleased to see the field of cultural psychology built on the basis of his methodological doubts.

\section{Philosophy and WEIRD intuition}

\section{doi:10.1017/S0140525X10000257}

\section{Stephen Stich \\ Department of Philosophy, Rutgers University, New Brunswick, NJ 08901-1107. \\ sstich@ruccs.rutgers.edu \\ http://www.rci.rutgers.edu/ stich/}

Abstract: From Plato to the present, philosophers have relied on intuitive judgments as evidence for or against philosophical theories. Most philosophers are WEIRD, highly educated, and male. The literature reviewed in the target article suggests that such people might have intuitions that differ from those of people in other groups. There is a growing body of evidence suggesting that they do.

In the opening pages of Plato's Republic, Cephalus suggests that what justice requires is speaking the truth and paying one's debts. But Socrates immediately offers a thought experiment to show that Cephalus's account of justice is not correct:

Suppose that a friend when in his right mind has deposited arms with me and he asks for them when he is not in his right mind, ought I to give them back to him? No one would say that I ought or that I should be right in doing so, any more than they would say that I ought always to speak the truth to one who is in his condition. (Plato 1892, The Republic, Book I, p. 331)

When Cephalus agrees, Socrates concludes that "speaking the truth and paying your debts" is not an adequate account of justice.
Philosophy has changed in many ways in the 2,400 years since Plato wrote the Republic, but the method Plato uses in this passage is still one of the most basic tools in the philosopher's toolkit. Although there is some debate about how, exactly, the method should be characterized, the basic outlines are clear enough. A philosopher describes a real, or more often an imaginary, situation and asks his audience whether the people or objects or events described have some philosophically important property or relation: Is the agent's action unjust? Was it morally wrong to push the large man off the footbridge to stop a trolley that would otherwise kill five people? (Thomson 1976) Does the person who holds a ticket in a lottery where the odds are one in a thousand know that the ticket won't win? (Kyburg 1961, p. 197; see also Hawthorne 2004). Does the "Chinese Room" understand the story? (Searle 1980) When things go well (as they always did in Plato's dialogues!), the audience will agree that the answer is intuitively obvious, and the philosopher will conclude that the content of these intuitive judgments is probably true. If it is intuitively obvious that it is wrong to push the man off the footbridge, and that is what the philosopher's theory entails, then this counts as evidence for his theory. If it is intuitively obvious that the Chinese Room does not understand the story or have other intentional states, but a philosopher's theory entails that it does, this counts as evidence against his theory.

The overwhelming majority of philosophers who use this method are WEIRD. Moreover, as reflected in my choice of pronouns, the majority of those who teach in North American and European philosophy departments are male. And, of course, on at least one dimension, professional philosophers are extreme outliers among WEIRD people, as most of them have undergone five or more years of training and vetting in one of the 30 or 40 leading graduate programs. About a decade ago, as we became acquainted with the emerging literature reviewed by Henrich et al., my colleagues and I began to wonder whether these WEIRD philosophers might have weird philosophical intuitions - intuitions that differed from those of people who did not share their cultural and educational background. To find out, we did something philosophers often do - we scoured the literature. We also did something that philosophers rarely do: We designed and ran our own experiments aimed at finding out whether people who were not WEIRD shared the intuitions that play such a central role in Western philosophy.

Although this work is still in its infancy, I think it has begun to provide an important addendum to Henrich et al.'s survey suggesting that, in a number of areas of philosophy, the intuitions of philosophically trained, WEIRD males are indeed quite different from the intuitions of people in other cultural groups. Space does not permit an exhaustive discussion of the literature, but here are some of the highpoints.

Epistemology. 1. Weinberg et al. (2001) and Nichols et al. (2003), showed that American students of European ancestry and American students of East Asian ancestry have different intuitions about a variety of thought experiments that have played a central role in contemporary philosophy. They also report differences in intuitions between high- and lowsocioeconomic status (SES) participants, where years of education was the major determinant in classifying a participant as high or low SES.

2. Starmans and Friedman (2009) found a large gender difference in intuitions on a "Gettier" case similar to those that are widely discussed in the philosophical literature. In recent years, there has been growing concern about the under-representation of women in philosophy (Haslanger 2008). Though the phenomenon almost certainly has many causes, this striking finding suggests one that most philosophers have been loathe to consider: Many women students may have intuitions that differ from those their male professors insist are correct.

Ethics. 1. The classic work of Brandt (1954) reports some dramatic differences between the moral judgments of Hopi people and white Americans that apparently cannot be explained by differences in factual beliefs.

2. Abarbanell and Houser (in press) report that in a variety of carefully controlled experiments, rural Mayan participants did not exhibit the 
"omission" bias that has been widely documented in a number of largescale societies.

Philosophy of language. Machery et al. (2004) report that Hong Kong Chinese and Americans of European ancestry have different intuitions on a thought experiment that has played a central role in the philosophical theory of reference. Deutsch et al. (in preparation) reinforce these findings, and Machery et al. (2009) include a preliminary report of additional studies in Mongolia, India, and France. (For further discussion, see Machery's commentary.)

There is no shortage of debate about the robustness of these findings and about their implications for the viability of the venerable philosophical practice of using WEIRD people's intuitions as evidence (Mallon et al. 2009; Sosa 2009; Stich 2009). My own view is that these studies pose a major challenge to that practice, because, when the intuitions of different groups diverge, there is no reason to think that WEIRD people's intuitions are more likely to be true.

\section{Authors' Response}

\section{Beyond WEIRD: Towards a broad-based behavioral science}

\author{
doi:10.1017/S0140525X10000725
}

\section{Joseph Henrich, ${ }^{a, b}$ Steven J. Heine, ${ }^{a}$ and Ara Norenzayan ${ }^{a}$ \\ ${ }^{a}$ Department of Psychology, University of British Columbia, Vancouver V6T $1 Z 4$ Canada; ${ }^{\mathrm{b}}$ Department of Economics, University of British Columbia, Vancouver V6T 1Z4, Canada. \\ joseph.henrich@gmail.com \\ http://www.psych.ubc.ca/ henrich/home.html \\ heine@psych.ubc.ca \\ ara@psych.ubc.ca}

Abstract: In our response to the 28 (largely positive) commentaries from an esteemed collection of researchers, we (1) consolidate additional evidence, extensions, and amplifications offered by our commentators; (2) emphasize the value of integrating experimental and ethnographic methods, and show how researchers using behavioral games have done precisely this; (3) present our concerns with arguments from several commentators that separate variable "content" from "computations" or "basic processes"; (4) address concerns that the patterns we highlight marking WEIRD people as psychological outliers arise from aspects of the researchers and the research process; (5) respond to the claim that as members of the same species, humans must have the same invariant psychological processes; (6) address criticisms of our telescoping contrasts; and (7) return to the question of explaining why WEIRD people are psychologically unusual. We believe a broad-based behavioral science of human nature needs to integrate a variety of methods and apply them to diverse populations, well beyond the WEIRD samples it has largely relied upon.

Frankly, we are stunned. We expected that our target article would provoke ferocious counter-attacks among a substantial cross-section of researchers from several fields. Awaiting the commentaries, we steeled ourselves, bracing for harsh and relentless rebukes. One renowned social psychologist, who had read an early draft, warned us that our colleagues would probably spit on us. What arrived were 28 commentaries from an esteemed and diverse set of scholars, including anthropologists, economists, linguists, neuroscientists, philosophers, primatologists, and sociologists, as well as cognitive, developmental, personality, and social psychologists. These commentaries largely cohere as an emerging synthesis, offering important expansions and extensions of our argument, as well as raising several interesting points for debate and discussion. There is now sufficient evidence from diverse human populations to indicate that researchers can no longer continue to - explicitly or implicitly - infer the universality of psychological processes or behavior from studying only WEIRD people and their children. Our reading indicates that 23 of 28 commentaries largely support our main thesis, although they raise important issues and fruitful points for debate. Of the remaining five, only one is in decisive disagreement (Gaertner, Sedikides, Cai, \& Brown [Gaertner et al.]), with the other four (Khemlani, Lee, \& Bucciarelli [Khemlani et al.], Machery, Maryanski, and Shweder) seeming somewhat ambiguous or ambivalent as to their precise views. Of course, it is possible that those who disagree most strongly with our assessment chose not to comment. We look forward to engaging representatives of this position in the future.

Our reply is ordered as follows: We (1) consolidate the additional lines of evidence, extensions, and amplifications of our target piece made by various commentators; (2) discuss the importance of integrating experimental and ethnographic methods, and show how researchers using behavioral games have done precisely this; (3) present our concerns with arguments from several commentators that separate "content" and "representations" from "computations," "learning," or "basic" psychological processes; (4) address concerns that the patterns we highlight marking WEIRD people as psychological outliers arise from aspects of researchers and the research process; (5) respond to Gaertner et al.'s claim that being members of the same species means we must have the same invariant psychological processes; (6) address criticisms of our categories and rhetorical strategy; and (7) return briefly to the question of explaining why WEIRD people are psychologically unusual.

\section{R1. Additional evidence, extensions, and amplifications}

Here we consolidate additional evidence, extensions, and amplifications of our target article. Seven commentaries reviewed empirical evidence that we did not present. All of this evidence supports the notion that WEIRD people are unusual, and none of it challenges that claim. Several of these lines of evidence are complementary with each other, and suggest some theoretical reasons for the unusual nature of WEIRD people, an issue that we return to in the final section. Nine additional commentaries supplied insightful amplifications, nuances, or extensions of our efforts.

\section{R1.1. Additional support for the argument that WEIRD populations are unusual}

1. Chiao \& Cheon point out that the vast majority of cognitive neuroscience findings are based on WEIRD brains. They then review findings from the nascent field of cultural neuroscience showing how population-level differences in experimental findings reveal themselves in 
brain activity. Some of the psychological differences we discussed regarding the self and holistic versus analytic reasoning can be observed in differential patterns of brain activation. These commentators also highlight differences in brain activation based on socioeconomic status. Their commentary underscores the point that neither imaging brains nor measuring hormones allows one to avoid the challenge of population-level variation. Moreover, because sophisticated cultural learning is an aspect of the evolved human repertoire, our brains are partially self-programmable, so even cultural differences are biological differences (though not genetic differences) - for example, see Nisbett and Cohen (1996).

2. Majid \& Levinson emphasize the importance of considering the world's immense linguistic diversity for studying and theorizing about psychology, especially in light of the unusual nature of English on several important dimensions (e.g., see next item). They also highlight additional evidence indicating how deeply some aspects of language imprint themselves on nonlinguistic aspects of cognition. They point to the uncanny coincidence that the fundamental stock of prelinguistic concepts hypothesized by cognitive scientists corresponds closely to those available in English - the language of many of the researchers and most of the participants - but not to those found in other languages.

3. Amplifying Majid \& Levinson's points with further examples, Machery and Stich discuss studies in America, France, Mongolia, India, and China showing that people have different philosophical intuitions, with English-speakers (and Americans) at one end of the spectrum and Chinese at the other. Apparently, some philosophical theories of reference are based on these "English intuitions."

4. Stich, in considering the implications of our efforts for philosophy, highlights novel work from experimental philosophy showing population-level differences in philosophical intuitions and moral judgments, including a recent finding on the lack of any "omission bias" in the moral judgments of Mayans. This lack of omission bias contradicts previous claims of universality based on work done purely in industrialized populations (and on the Internet). These initial findings, if replicated and extended, suggest that important elements of philosophical theorizing and conceptual analysis are rooted in local folk intuitions that do not extend to the rest of humanity.

5. Karasik, Adolph, Tamis-LeMonda, \& Bornstein (Karasik et al.) review evidence on differences in motor development across human populations, and recent data linking developmental differences to childrearing practices. They also highlight how, in some cases, different developmental trajectories arrive at the same outcome.

6. Fernald, in underlining the extreme narrowness of the samples used by developmental psychologists, reviews evidence linking socioeconomic status, early cognitive stimulation, and long-term cognitive outcomes. Since most developmental work is with infants from WEIRD families, the oft-highlighted developmental milestones for various cognitive and linguistic abilities may reside at the extreme end of the true underlying species distribution.

7. Lancy reviews both ethnographic and experimental work on children from across diverse human populations showing just how unusual the worlds are of children who grow up in WEIRD societies, and how different their cognitive development can be.

\section{R1.2. Extensions of our points}

Here we lay out five different ways in which various commentators highlighted, amplified, and extended our efforts.

R1.2.1. Dealing with the predominance of WEIRD researchers. One important issue that we were not able to spend much space on in our target article revolves around the fact that most researchers are themselves WEIRD people. This directly impacts the choice of subject pools, since many researchers study those around them. However, this also may impact theory building and experimental design in a number of ways (e.g., Majid \& Levinson, Stich). First, Fessler emphasizes how researchers use their own intuitions, at least at the start, in either theory-building or experimental design. His discussion of research on shame shows how American cultural models of emotion lead to basic elements of this emotion being missed by U. S. researchers, despite the fact that these elements are highly salient elsewhere. He argues that researchers, at least for some topics, would be better off not studying people who are culturally similar to themselves. Second, Bennis \& Medin worry about this same point, arguing that researchers' cultural biases influence the choice of topics and phenomena that are considered interesting. Further magnifying the problem, instruments are then developed and honed in particular populations, which may not be suitable to other populations (also see Rochat). Finally, Meadon \& Spurrett suggest that one important way of addressing these challenges is to bring more non-WEIRD researchers into the process. Empirical findings should be peer reviewed by researchers who bring different cultural models and implicit expectations to the problem.

We agree with all these suggestions: Researchers can view phenomena from a novel perspective, not constrained by their own intuitions, when they study those from other cultures, and can potentially discover phenomena that they otherwise would not see. However, we disagree with an extreme version of this argument, which proposes that researchers should entirely avoid studying people from their own culture. Researcher's intuitions about the ways people in their own cultures think can be a useful source of understanding in building theories and in honing research instruments. More non-WEIRD researchers should be brought into the discussion, as well as onto collaborative research teams. Research teams themselves that better reflect broad global diversity can more effectively address the challenges delineated by Fessler, Rochat, and Bennis \& Medin.

With regard to these points, it is instructive to consider why psychology is more dominated by American research than any other science (May 1997). One possibility is that pursuing a career in psychology is a luxury that people cannot afford until the countries and societies in which they live have achieved sufficient economic development. This may be part of the explanation, although this would not explain why universities in wealthy societies like those of Japan and Western Europe typically have 
proportionately smaller complements of psychology researchers and majors than do North American universities. Another possibility, which we highlight here, is that the field's emphasis on WEIRD samples, coupled with the guiding assumption of universal psychological processes, tends to unintentionally marginalize international research. If non-WEIRD researchers are interested in extending findings initially established with WEIRD samples in their home populations, such as findings associated with motivations for self-enhancement, they may well be unable to replicate the American results. The implicit assumption that self-enhancement motivations are similar everywhere would suggest that such failed replications are not due to the nature of the samples studied but instead due to some kind of unspecified deficiency in the methods of the non-WEIRD researchers. American researchers have a distinct advantage in that the field's key theories were largely constructed on data from American participants, and we suggest that this is likely why American research constitutes $70 \%$ of the field's citations. International research suffers from the disadvantage of trying to extend American-based theories with participants who often have different psychological tendencies, yielding results that are difficult to interpret while embracing an untested assumption of universal psychological processes. In contrast, if the field comes to recognize that psychological phenomena cannot be assumed to be universal until demonstrated as such, then research conducted by non-WEIRD researchers, guided by non-WEIRD intuitions, and studied with non-WEIRD samples, would come to be viewed as particularly important for understanding human psychology.

R1.2.2. Existential proofs. Gächter's commentary also extends one of our discussion points by underlining the fact that, depending on the research question, WEIRD subjects may be suitable, or even ideal. In our target article we wrote that

Research programs that are seeking existential proofs for psychological or behavioral phenomena, such as in the case of altruistic punishment discussed earlier (e.g., Fehr \& Gächter 2002), could certainly start with WEIRD samples. That is, if the question is whether a certain phenomenon can be found in humans at all, reliance on any slice of humanity would be a legitimate sampling strategy. (sect. 7.1.6)

We pointed both to Kahneman and Tverky's work on rationality (e.g., Gilovich et al. 2002) and to Rozin's work on magical thinking (Rozin \& Nemeroff 1990) to highlight situations in which WEIRD samples are either suitable or ideal. However, if one's goal is ultimately to construct (rather than tactically falsify) theories of human behavior, it is hard to see how that could be done without expanding beyond WEIRD subjects.

R1.2.3. Differences among chimpanzee populations. Two commentaries expand on one of our points (Note 14 of the target article) by highlighting the challenge that population-level psychological variation creates for programs comparing humans and chimpanzees (Boesch and Leavens, Bard, \& Hopkins [Leavens et al.]). Applying our argument to chimpanzees (though not to other animals?), these commentaries make the point that chimpanzee populations may also vary in their psychological abilities and motivations, and that this difference is important for comparing wild and captive populations.

Broadly speaking, we agree with this point and feel it needs careful attention. Nevertheless, we offer some cautionary notes. First, there are both theoretical and empirical reasons to believe that human population-level psychological variation is substantially greater than that found in chimpanzees. While chimpanzees are a cultural species, with local traditions and some imitative abilities (Horner \& Whiten 2005; Whiten et al. 1999), humans are a runaway hyper-cultural species whose genetic endowments, including abilities to adapt ontogenetically, have been shaped by a long history of cumulative cultural evolution, social norms, institutions, and culture-gene coevolution (Henrich 2008; Laland et al. 2010; Richerson \& Boyd 2005). With the same basic genetic endowments, humans expanded as foragers to all major continents, across substantial bodies of ocean, and into an immense diversity of environments. Meanwhile, chimpanzees remained stuck in a narrow band of African tropical forests. The impact of culture-gene coevolution has become increasingly clear from studies of the human genome (Laland et al. 2010). Therefore, although we agree that understanding chimpanzees also requires the study of diverse samples, we suspect that populationlevel variation is a far more significant issue for understanding human psychology than for understanding chimpanzee psychology.

Second, we note that it is far from clear which way the use of captive chimpanzees in psychological experiments might bias empirical findings. The aforementioned two commentaries provide opposing claims on this issue. Boesch suggests that if populations of human foragers were compared with wild chimpanzees, then the observed psychological and motivational differences would be minimized because of the impoverished social environments of captive chimpanzees and the unusual psychology of WEIRD people. Leavens et al., somewhat contrastingly, summarize evidence indicating that captive and humanreared chimpanzees have declarative pointing abilities more similar to humans (or at least to their human captors) than is found in wild chimpanzees. This suggests that captive chimpanzees may be more similar to some humans than to wild chimpanzees. It certainly seems plausible that life with humans might make chimpanzees more similar to humans, not less. This remains an open and important question, the answer to which is likely to vary depending on the phenomenon under investigation.

Finally, some of Boesch's specific indictments are off the mark. For example, he suggests that Silk et al. (2005) designed experiments with the "ethnocentric assumption that sharing should be preferred over nonsharing," and then affirmatively cites Henrich et al.'s (2006) work, which argues that these differences result from different culturally evolved norms. It turns out, however, that Henrich was a co-investigator on both projects, and that these chimpanzee experiments were designed with full knowledge of the cross-cultural results, and precisely to test the "cultural norms" hypothesis against alternatives. Moreover, these experiments were done with chimpanzees in Louisiana, which Boesch criticizes, but were also replicated in Bastrop, Texas, prior to publication (Silk et al. 2005), and then replicated again in Leipzig (Jensen 
et al. 2006). Despite the substantial variation in the social environments of these chimpanzee populations, the experimental results were identical in all three sites. Finally, although differences between captive and wild chimpanzees may be important, there is nothing inconsistent about field observations of chimpanzee cooperation and Silk et al.'s experimental results. Pure self-interest can generate plenty of sharing and cooperation in some contexts, and seems to explain much about chimpanzees' social behavior (Gilby 2006; Tennie et al. 2009).

R1.2.4. Generalizing across contexts. The commentaries by both Konečni and Ceci, Kahan, \& Braman (Ceci et al.) call attention to another concern regarding generalizability: How well do research findings generalize beyond the methods that are used to test specific hypotheses in laboratory settings? Relatedly, Rochat argues that the choice of narrowly conceived psychological instruments may limit the generalization of findings, as well. We agree with Konečni, Ceci et al., and Rochat that there are potential artifacts underlying the findings of many experimental paradigms. Highlighting the need to broaden participant samples does not obviate the need for researchers to study their phenomena with a variety of methods and in different contexts to assess whether their findings are meaningful and generalizable.

R1.2.5. Implications beyond the laboratory. The unusual nature of WEIRD samples is not solely a problem for researchers; it has implications that extend well beyond the laboratory. We think that Konečni is correct in highlighting how automatic assumptions of psychological universality can be problematic when people from one society apply and enforce new norms and policies in another society. As he notes, there can be enormous costs in "the deliberate or unconscious incorporation of WEIRD-based findings into the normative expectations held by international bodies in 'cognitively distant' wartorn areas - such as in Rwanda." International interventions that are based on WEIRD research, or inspired by untested universalist assumptions, may generate ineffective and potentially destructive policies.

We emphasize, however, that an awareness of population variability is not a call for unbridled cultural relativism. Findings that reveal population differences do not imply an absence of a universal human nature, but they do indicate that what is universal might not be the same as what emerges from WEIRD participants. The investigation of universals can play a central role in the endeavor to manage international disputes and humanitarian crises, because they stand to possibly provide the only legitimate criteria by which any particular cultural practice or belief system may be understood. As Fox (1973, p. 13) has argued, "We could not plead against inhuman tyrannies if we did not know what is inhuman." Understanding what is human or inhuman necessarily requires studying people from a diversity of populations.

Theories based on narrow sampling also have disturbing implications for the field of psychiatry and the treatment of mental health across diverse cultural contexts. As in the behavioral sciences, psychiatric models have largely been constructed on an empirical foundation that was gathered from WEIRD people. The burgeoning field of cultural psychiatry (Tseng 2001), however, has revealed that many disorders have distinct cultural boundaries, and can best be understood as culture-bound syndromes, such as (1) bulimia nervosa in the West (Keel \& Klump 2003), (2) hikikomori in Japan (Sakai et al. 2004), and (3) koro in Southeast Asia (Ngui 1969). Moreover, many universal mental disorders manifest themselves in quite distinct ways across populations, such that presentations of depression (Kleinman 1988), social anxiety disorder (Okazaki 1997), or even schizophrenia (WHO 1973) are associated with different symptoms and prognoses. In his recent book, Crazy Like Us: The Globalization of the American Psyche, Watters (2010) documents how psychiatry has been exporting American models of psychopathologies around the world, such as post-traumatic stress syndrome to Sri Lanka, anorexia nervosa to Hong Kong, and depression to Japan, often with disastrous consequences. The problem lies in diagnosing and treating indigenous presentations of pathologies according to how they appear through the prisms of the culturally limited diagnostic categories of the American Psychiatric Association's Diagnostic and Statistical Manual of Mental Disorders, which often increases the distress for both the patient and the community. When real-life interventions are based on a body of research - however extensive - that is disproportionately drawn from WEIRD samples, the implications extend far beyond the accuracy of our theories and into human lives.

\section{R2. Methods for an interdisciplinary science of human behavior}

We agree with Rai \& Fiske, Astuti \& Bloch, and Shweder that a fully interdisciplinary study of human psychology demands an integration of ethnographic and experimental methods. Panchanathan, Frankenhuis, \& Barrett (Panchanathan et al.) also recognize the need for research that goes beyond disciplinary boundaries. Experimental methods provide instruments for better measurement and permit the testing of causal hypotheses. Ethnographic methods provide crucial insights for developing theory, designing experiments, and interpreting results, as well as important information for understanding the proximate causes (e.g., ontogenetic processes) of psychological differences (Henrich \& Henrich 2007, Ch. 1). However, because our target article was aimed principally at experimentalists, we wrote in the language of experiments. Too often, ethnographers have railed against experiments, but little communication has occurred because ethnographers generally have refused to become fluent in the local language of experimental thought (which is ironic).

There are important differences here, however. Our own view is more in line with Rai \& Fiske, than with Astuti \& Bloch and Shweder, who seem to be emphasizing an approach based on qualitative ethnography with an emphasis on "thick description." Ethnographic work must be based on systemic, quantitative, and replicable research protocols that quantify the theoretically relevant aspects of life. Alongside in-depth interviews and participant observation, this might involve time allocation, systematic observation, social network measures, conversational recordings, and formal cognitive tasks (e.g., pile sorts). The integration of experimental techniques with ethnography will partially return anthropology to its broader scope, 
prior to the intellectually destructive epidemic of postmodernism (Slingerland 2008). As Shweder points out, field anthropologists used to integrate experiments with ethnography (e.g., Edgerton 1971; Mead 1932; Rivers 1901b). What we do not need is greater reliance on ethnographic impressionism, which delivers such spectacles as the Mead-Freeman-Orans debate (Freeman et al. 2000) on the nature of Samoan adolescent sexuality.

In the 21st century, scattered teams of highly interdisciplinary researchers have already begun to demonstrate how to integrate ethnographic and experimental findings in a manner that takes advantage of their synergies, going well beyond "thick description" (e.g., Atran et al. 1999; 2005; Barrett \& Behne 2005; Cohen 2007; Fessler 2004; Henrich et al. 2005a; 2006; Henrich \& Henrich 2007). In Section R2.1 below, we discuss Foundations of Human Sociality: Economic Experiments and Ethnographic Evidence from Fifteen Small-Scale Societies (Henrich et al. 2004), which explicitly integrates insights from long-term fieldwork with findings from behavioral games.

\section{R2.1. Meanings and misunderstandings: Behavioral game experiments}

In expressing concerns about how experimental participants from diverse societies interpret or conceptualize particular experiments, some commentators have criticized the use of "economic games" across diverse societies

(Shweder, Rai \& Fiske, Baumard \& Sperber, and Astuti \& Bloch). We address these specific issues in two ways. First, we show how these criticisms arise from an incomplete reading of the work that has been done using economic games across diverse populations. Second, we use these cross-cultural game projects as an example of how in-depth ethnographic studies can be combined with experimental tools by interdisciplinary teams to address important theoretical questions.

Understanding the utility of an experiment requires understanding the theoretical debates that those experiments aim to address. The Roots of Human Sociality Project, which consists of two phases of experiments and ethnography performed in 22 different small-scale societies by a team of anthropologists, psychologists, and economists (see Henrich et al. 2001; 2006; 2010), was designed to examine particular hypotheses related to the evolution of large-scale complex human societies. One hypothesis for the emergence of large-scale human societies proposes that cultural evolution, driven by competition among societies and institutions, favored the evolution of particular kinds of social norms. These norms harness and extend evolved social motivations to foster cooperation, trust, and exchange with ephemeral partners, beyond each individual's stable local network of kin and repeat interactants. Such norms permitted the formation of market institutions, which encourage market expansion, trade, and economic success. Psychologically, this hypothesis suggests that the inhabitants of large-scale, complex, market-integrated societies will possess default sets of prosocial beliefs, motivations, and expectations about how to treat ephemeral interactants (e.g., strangers or anonymous others). Under this view, the institutions of complex societies, such as markets, ought to correlate positively with prosocial behavior in these contexts.
An alternative hypothesis proposes that cooperation, trust, and exchange in large-scale societies result directly from the misapplication of evolved kin- and reciprocitybased heuristics for interacting in small-scale societies to individuals in larger social spheres (Burnham \& Johnson 2005; Dawkins 2006), eventually including nation states. These heuristics for life in small-scale societies, which are not favored by natural selection in large-scale societies, misfire in large-scale societies because these societies have spread only in the last 10 millennia. Crucial to this hypothesis is that cultural evolution cannot substantially alter the social motivations and calculations that determine sociality.

These two hypotheses make quite different predictions about the context-specific behavior and motivations of people from different societies toward ephemeral interactants.

What kind of experiments might allow us to measure these differences? Ideally, the experiments should have real costs and benefits with the same underlying material payoffs, so we can comparatively measure motivations and expectations. However, there ought to be cues that will tap the predicted sets of context-specific motivations and expectations (norms) for interacting with individuals in the absence of information about their particular relationships (e.g., cues about status, sex, kinship, or future interaction). With their salient cues of cash and anonymity, and their lack of other cues, economic games seem ideally suited for testing the above hypotheses (Henrich et al. 2010).

From the beginning, the Roots team leaders knew that deploying these experiments across diverse societies would be challenging, requiring experts on each local culture and qualitative ethnographic information to assess local meanings and interpretations. Long-term anthropological fieldworkers were recruited to design and implement the protocols. Although the Phase I findings did show that market integration was indeed important for predicting prosociality in these contexts, there were also a few cases in which the experiments happened to cue local prosocial norms - interpretations or meaning systems - unrelated to the targeted set of default norms for exchanging with strangers or anonymous others. The project team attended to these alternative interpretations, arguing that it is essential to understand the mapping between the experiments and local norms (Alvard 2004; Ensminger 2004; Henrich \& Smith 2004; Hill \& Gurven 2004); they also captured much of this variation with a variable related to non-market cooperative domains in their statistical analyses. The observation of how daily life influences the experiments was so important that it was one of the five major points in the team's 2005 BBS paper (Henrich et al. 2005a). Each ethnographer also wrote a chapter in Foundations of Human Sociality: Economic Experiments and Ethnographic Evidence and in Fifteen Small-Scale Societies, in which they deployed their own interviews, participant observation, and years of ethnographic experience to illuminate the local meanings of our experiments.

An important example of this focus on understanding local meanings comes from the team's investigation of their experimental findings from the Au of New Guinea (see Shweder). This investigation began when ethnographer David Tracer, who speaks Au and had been 
working in New Guinea for 12 years prior to the Roots collaboration, published two papers illuminating his experimental findings (Tracer 2003; 2004). To further address this in Phase II, the Roots team recruited another longterm linguistically skilled New Guinea ethnographer, Alex Bolyanatz. In this second phase, Tracer replicated and extended his prior findings, while Bolyanatz returned home with findings parallel to those of Tracer (Bolyanatz, under review; Tracer et al., under review). It seems likely that in New Guinea, behavioral games map onto prosocial norms that have little or nothing to do with markets or complex societies. This is consistent with decades of ethnography emphasizing the broad-ranging importance of reciprocity norms in New Guinea (Fiske 1991; Sillitoe 1998).

After the Phase I findings became known, many researchers expressed the same concerns that have been highlighted by commentators Baumard \& Sperber, who write "participants in these games have no information about the rights of each player over the stake and are asked to make a 'blind' decision. But who owns the money? ... Who is the other participant? ... Does he or she have rights over the money?" Whereas the Roots team argued against these concerns for Phase I (Henrich et al. 2005b), Phase II's design directly and explicitly addressed them in the standardized game instructions, pre-game tests of participants' understanding, post-game interviews on game interpretations, contextualized game variants, and games with double-blind anonymous partners. Phase II's results replicated and extended the Phase I findings in various ways, showing that, among other things, the modifications made to address the concerns raised by Baumard \& Sperber have little impact on the results (Henrich \& Ensminger, n.d.; Henrich et al. 2006; 2010).

Rai \& Fiske suggest the experimental games are not meaningful because they do not correlate with anything important in the real world (Levitt \& List 2007). They cite Gurven and Winking (2008), who show that socializing, food-sharing, beer-brewing, and well-digging are not correlated with three bargaining experiments among the Tsimane in Bolivia. We question what theory predicts that those domains should be correlated? The above described evolutionary approach to social norms predicts that, if game play does indeed tap norms evolved for interacting with strangers or anonymous others, then the games played by Gurven and Winking ought to be associated with things such as market integration, social scale (community size), and other features related to the operation of largerscale societies - features that capture those elements of social interactions not governed by durable personal relationships. Looking across diverse populations, market integration is indeed highly correlated with experimental measures of prosocial behavior in these bargaining games (Henrich et al. 2010). Similarly, antisocial punishment (Figure 3 in the target article) is highly negatively correlated with GDP (gross domestic product), and predicted by national measures of the strength of the rule of law and measures of norms of civic cooperation (Herrmann et al. 2008). Within populations, trust game measures of trustworthiness predict repaying loans in a microfinance program (Karlan 2005), and predict alumni donations (Baran et al. 2009). Dictator game offers are correlated with donations to hurricane victims (Kam et al., n.d.) and political participation (Fowler \& Kam 2007).
Once properly theorized, not only are economic games highly correlated with important real-world phenomena, but we can predict with which real-world phenomena they should correlate.

\section{R2.2. Merely methodological artifacts?}

Beyond economic games, Shweder, as well as Baumard \& Sperber, ask whether the population-level variations found in the psychological literature we reviewed are a product of methodological artifacts arising from different meanings assigned to the experimental settings, or from communication failures between researchers and participants. Could it be that, for example, the extensive cognitive differences we reviewed in perceptual judgments, visual illusions, analytic-holistic thinking, and folkbiological reasoning arise not from differences in cognitive processes, but from different interpretations of the questions or the tasks?

While we agree that researchers working across populations should take such methodological concerns seriously, several lines of evidence speak against this being a general problem. First, we emphasize that diverse methodological techniques have often been used that yield consistent findings. For example, Western participants are found to privilege analytic cognitive strategies, whether the tasks measure reaction times in categorization, free recall, patterns of bias in deductive reasoning, or eye tracking in scene recognition. It is hard to see how task interpretation or meaning issues would affect all of these tasks and yield responses in the same direction as the predicted differences in cognitive processes. Moreover, many of the psychological studies we included in our review (Choi \& Nisbett 1998; Norenzayan et al. 2002) included control conditions in which there were no population-level differences, and none were expected. This helps establish the meaning equivalence of the experimental contexts and procedures across populations, and undermines a purely methodological interpretation of differences found. Finally, it is important to recognize that the same or similar methods and instruments that have revealed population-level differences have also revealed population-level invariances, often in the same study with the same participants (Atran \& Medin 2008; Atran et al. 2005; Haun et al. 2006; Henrich et al. 2006; Norenzayan et al. 2002; Segall et al. 1966). As we point out in our target article, if certain methods count toward establishing invariant aspects of psychological processes, then data that indicate variation have to count as well.

As an illustration, consider recent work showing both universal and variable aspects of numerical cognition. In contrast to evidence from WEIRD samples, experimental work from two small-scale Amazonian societies, the Piraha and the Munduruku, suggests that the ability to distinguish quantities digitally beyond the first couple of integers is poor in these groups, whose languages do not include numerals above 3 (Gordon 2004; Pica et al. 2004) - a pattern common in many such societies (Everett 2005). These same experiments also demonstrate that the cognitive ability to estimate quantity approximately, or an analog "number sense" (Dehaene 1997), is found to be strikingly similar irrespective of linguistic variation in counting systems. This analog system is also present in numerous nonhuman species (Hauser \& Spelke 2004). 


\section{R2.3. Accessing non-WEIRD samples}

Commentators Gosling, Carson, John, \& Potter (Gosling et al.) discuss an important tool that should allow the behavioral sciences to obtain more diverse samples by reaching non-WEIRD participants through the Internet. We agree that this is a potent addition to the researcher's toolbox. The advantages of the Internet are the ease and affordability by which international samples can be accessed. We suspect that some fields are probably more likely to change in the ways we prescribe if the changes do not require researchers to alter their habitual practices, or leave their home universities to go out into the world.

While the Internet is undoubtedly a valuable tool that needs to be fully exploited, there are some limitations to this approach, as Gosling et al. note. First, though the Internet is amenable to some kinds of psychological experimentation, it will not facilitate the kind of integrated research program that synthesizes tools from across the human sciences, including direct observation, naturalistic field experiments, biomarkers, and qualitative ethnography, into longitudinal studies across the life cycle. Second, the segments of many countries that have Internet access probably share many attributes with WEIRD people already (see Rozin) - they will tend to be rich, educated (at least literate), and often disproportionately from particular ethnic groups. For example, Internet users in Africa are far more likely to be cultural outliers in WEIRD ways relative to the general African population, compared to, say, Internet users in Europe relative to the general European population. Third, many people overestimate the current reach of the Web. Our Table R1 gives the percentage of the total population in each of the world's major regions that comprises of Internet users (i.e., percentage of Internet penetration across the globe). The percentages for Africa, Asia, and Latin America are not only low, but the distributions are highly skewed. Most African countries have Internet penetrations of less than 1\%; and the overall African penetration is distorted upward by the higher distribution in Egypt, South Africa, and Nigeria. The aggregate statistics

Table R1. Global distribution of Internet penetration as of $2009^{1}$

\begin{tabular}{lrrr}
\hline \hline World & \multicolumn{1}{c}{$\begin{array}{l}\text { Internet } \\
\text { Regions }\end{array}$} & $\begin{array}{l}\text { Population } \\
\text { Users }\end{array}$ & $\begin{array}{l}\text { Pof } \\
\text { Population }\end{array}$ \\
\hline Africa & $991,002,342$ & $67,371,700$ & 6.8 \\
Asia & $3,808,070,503$ & $738,257,230$ & 19.4 \\
Europe & $803,850,858$ & $418,029,796$ & 52.0 \\
Middle East & $202,687,005$ & $57,425,046$ & 28.3 \\
North & $340,831,831$ & $252,908,000$ & 74.2 \\
$\quad$ America & & & \\
Latin & $586,662,468$ & $179,031,479$ & 30.5 \\
$\quad$ America/ & & & \\
$\quad$ Caribbean & & & 60.4 \\
Oceania/ & $34,700,201$ & $20,970,490$ & \\
$\quad$ Australia & & & \\
WORLD & $6,767,805,208$ & $1,733,993,741$ & 25.6 \\
$\quad$ TOTAL & & & \\
\hline \hline
\end{tabular}

for Oceania/Australia are also deceptive, as more than half the countries comprising that region have less than $15 \%$ penetration, with six countries showing less than $5 \%$ penetration (Solomon Islands, Kiribati, Papua New Guinea, Nauru, Marshall Islands, and Samoa). Fourth and finally, we hope that the ease of this method does not discourage researchers from considering other ways to broaden their research programs to integrate diverse populations. Overall, we are in favor of any additional methodological tools that can be used to study diverse human samples, and we believe the field will be best off by using a wide variety of different tools.

\section{R3. "Basic level” processes, learning, and computational operations}

Four commentaries emphasize a bipartite partition between (1) mental content and (2) basic or universal, psychological processes (Rozin), variously labeled as "learning" (Danks \& Rose), "computational operations" (Khemlani et al.), or "low-level processing" (Rochat). Each commentary emphasizes that while content varies, the underlying computational machine is constant. Danks \& Rose write, "there is a natural, defensible distinction between the cognitive 'objects' of the mind, and dynamic mental 'processes.' Cognitive objects include representations, knowledge structures, and so on." Similarly, Rozin notes, "But at the level of basic psychological processes, such as learning, motor organization, or vision, the NAU [North American undergraduate] is probably a pretty good fruit fly." Maybe this is how the human mind operates, like the computer on your desk, but how do we know until we sample a broad range of human diversity? Human learning or computational processes might be self-modifying to adapt to local conditions, so, while all human fetuses might begin with (roughly) the same cognitive equipment, acquired content could provide feedback and alter these "basic"-level learning or computational processes through phenotypic plasticity or cultural transmission. Or, culture-gene coevolution, which is increasingly recognized as a powerful force in human evolution (Laland et al. 2010), could genetically adapt local populations to more effectively acquire and process the local stable cultural representations.

Our own folk model of human psychology, which is also rooted in a computer metaphor, conforms to that suggested by these commentators. However, we think the available evidence ought to make us question this metaphor. Let's first consider vision, since Rozin highlights this. By all appearances, vision seems to be the product of "basic" processes. We have already discussed the population-level variation in the Müller-Lyer illusion, which, according to Khemlani et al., is "but one single phenomenon in visual perception, hardly representative of all visual perceptual processes." However, as we noted, there is also substantial variation in the Sander Parallelogram and two versions of the Horizontal-Vertical illusion.

But forget illusions. Suppose one was studying why people see so poorly underwater, compared to on land. Can one make universal generalizations regarding the human ability to see underwater by exclusively studying undergraduates? Turns out, no (Gislen et al. 2003). The 
Moken are nomadic sea foragers who live in an archipelago off the coast of Burma. From a young age, Moken subsist by collecting food from the sea floor. Comparisons of underwater visual acuity between Moken and European children show that Moken children have more than twice the visual acuity of their European counterparts. The Moken appear to have acquired the ability to constrict their pupils underwater, thus improving acuity, rather than widening them in the dimmer light, as Europeans do. That is, the pupils of Moken and Europeans do opposite things when they enter water, one adaptive, the other, not so much. Isn't pupil dilation a "basic" part of visual processing?

Rozin also mentions "motor organization" as a basic process. It is not clear to us precisely what this means, but Karasik et al.'s commentary reviews evidence showing how important aspects of motor development vary across human populations, and suggests how this might be related to childrearing or parenting practices. Karasik et al. also discuss how children in some smallscale societies never crawl - instead, they "butt-scoot" or "bum-shuffle." A "crawling stage" per se is neither universal nor necessary for adult bipedalism.

Perhaps a non-psychological example will further sharpen the problem. Suppose you wanted to study the nature of human running. Can you build a universal model of human running that is based on undergraduates, or other WEIRD people? Interestingly, WEIRD people would be one of the worst populations to select for such an investigation. Recent research shows that cushioned running shoes lead to dramatic modifications of the human running profile by causing runners to land principally on their heels instead of the balls of their feet. This difference has substantial implications for how we understand the evolved design and engineering of human feet (Lieberman et al. 2010). If one studies life-long sneakerwearers, the engineering of human feet appears ill-suited for long-distance running. In contrast, studying barefoot runners, particularly life-long barefoot runners, suggests a marvelous evolved design for the human foot, possibly specialized for long-distance running (Bramble \& Lieberman 2004). Hence, if you study WEIRD runners exclusively, you again get the wrong answer.

The difference between the feet of shod and unshod people has implications even for the interpretation of ancient hominid evolution. In 1978-79 a 27.5-meter-long trail of footprints hardened in volcanic ash dating to 3.5 million years ago was unearthed at Laetoli, Tanzania. Comparisons of these ancient prints with those of urban North Americans suggested that although bipedal, these ancient hominids were not bipedal in the way Homo sapiens are. The ancient footprints show a separation between the big toe and second toe, an anterior "fanning," and a substantial arch - all indicating differences compared to the feet of WEIRD humans. However, when the Laetoli prints were compared with those of the Machiguenga, who live a barefoot life of hunting, gathering, and horticulture in the Peruvian Amazon, the Laetoli footprints could not be distinguished from these non-WEIRD footprints (Tuttle et al. 1990; 1991). It turns out that WEIRD people have flat, narrow feet with underdeveloped big toes, which are the product of a lifetime of having one's feet bound in cushioned shoes. One cannot even safely identify universals about human foot anatomy by exclusively studying WEIRD people!
The assumption that "basic" processes are invariant also needs to stand up to evidence that human brains change in response to experience and cultural routines. Work on neuroplasticity has shown how training and expertise can create both functional and structural differences in brains. But the demands and incentives of developmentally adapting to local social organizations, status hierarchies, performance norms, carpentered corners, and other culturally evolved features of developmental environments persist for longer periods, are probably more constant, and are arguably more intensive than the behavioral regimes associated with occupations, such as those associated with musical training, mathematics, or taxi driving (Reynolds Losin et al. 2010). Yet, musical training creates structural alterations in brains, such as enlarging the anterior corpus callosum and altering the motor and somatosensory maps. Taxi driving increases gray matter in the hippocampus, while training as a mathematician increases grey matter in the parietal cortex. Consequently, however one conceives of these hypothesized invariant "basic processes," such processes would have to remain constant in the face of the structural and functional modifications in brains that inevitably arise from ontogenetically adapting to culturally constructed environments (Reynolds Losin et al. 2010). As Panchanathan et al. recognize, rather than demanding universal psychological processes, it might be fruitful to think about evolved ontogenetic processes that construct and calibrate diverse psychological processes to local environments, at least for some domains.

Recent evidence emerging from collaborations between cognitive psychologists and anthropologists further challenges the distinction between process and content that stands as a virtual axiom in parts of the cognitive sciences. Cultural differences in what people think about loops back to impact how people think (Bang et al. 2007). For example, in folkbiology, differences in what people believe about plants and animals affect memory organization and ecological reasoning about living things.

Finally, a key point that is missed by the prevailing arguments supporting a distinction between "universal process" versus "variable content" is that universality is not an all-or-nothing phenomenon. In order to draw meaningful conclusions about what is universal and what is not, one must also make distinctions between different levels of universals that are grounded in empirical observation. For example, the cognitive ability to estimate quantity approximately, discussed earlier, appears to be quite invariant in that it produces cognitive responses with identical effect sizes across populations. In contrast, many processes of central interest to psychology and cognitive science, such as rule-based categorization, geocentric spatial reasoning, or some egocentric motivational biases, are universal in a much weaker sense. They may exist in the psychological repertoires of all peoples, but their use and relative dominance over other competing strategies are contingent on population-level variability in cultural routines and practices. See Norenzayan and Heine (2005) for a theoretical framework for identifying levels of universals.

In sum, it is not clear to us what kinds of psychological processes are a priori more likely to be universal. More empirical evidence regarding the degree to which psychological phenomena vary across populations will be of much 
utility in addressing this important question. Perhaps, as Machery alludes, such evidence will reveal that social psychological phenomena (whatever those are?) are especially likely to vary across populations. But at this point, there is insufficient evidence to support such a conclusion. $^{2}$

\section{R4. Are WEIRD populations actually unusual?}

While agreeing with our central thesis, Bennis \& Medin worry that the seemingly extreme nature of WEIRD populations on many dimensions may result from various biases created by the fact that most researchers are themselves WEIRD, and that psychological instruments are developed and honed in WEIRD populations. We are sympathetic with their concerns, although we quibble with some of the details of their critique.

Bennis \& Medin begin their critique by proposing that a consideration of base rates would suggest that something is amiss with our claim. Their logic implies that it was essentially a random process that determined which society, of all historical and extant societies, happened to accumulate sufficient experimental findings about human behavior from enough populations to begin to consider the question at hand. However, the right way to think about the question they are posing is to ask: What is the probability that a society is psychologically unusual, given that it is the first to aggregate sufficient experimental findings from diverse societies to even explore the question? For starters, such a society has to inherit a scientific and experimental tradition. The society has to be economically successful enough to create occupational specializations for experts in human psychology and behavior. The society has to be willing to place sufficient value on these activities, despite their questionable economic utility. This society must be willing to fund data collection in some diverse populations. And, at least some members of the society have to find this endeavor sufficiently interesting to dedicate their lives to pursuing it. Given all these prerequisites, which limit the number of candidate societies to a handful, we think it is quite plausible that the society which first had sufficient experimental findings to explore the question at all, would itself be psychologically unusual.

In fact, many of the findings reviewed in our article and in these commentaries could plausibly be linked to being in a position to explore the question. Commenators Kesebir, Oishi, \& Spellman (Kesebir et al.) note that Americans have low pathogen loads and high residential mobility, both of which are correlated with individualism and may promote economic growth. Together, Lancy and Fernald suggest that American (and Western, more generally) childrearing practices may speed the cognitive development of particular skills. We pointed out the unusual lack of co-sleeping in the United States, which may influence independence and self-reliance. Individualistic notions of the self may increase Americans' curiosity about psychology, which might explain why psychology is so dominated by U.S.-based research. Differences in holistic versus analytical thinking may be linked to epistemic social norms about what counts as "good thinking" (Buchtel \& Norenzayan 2008), and may be rooted in the very origins of science (Nisbett 2003). Visual illusion variability and folkbiological anomalies may be linked to growing up in built urban environments with ample twodimensional representations (artwork, photographs). Motivations for fairness towards anonymous others are highly correlated with market participation, whereas motivations for antisocial punishment strongly negatively predict GDP; both of these factors can impact economic performance. In short, it appears that a society that has conducted and amassed a large majority of the extant psychological data may, for precisely the same underlying reasons, be a psychological outlier, at least on many important dimensions. These are not independent processes, as Bennis \& Medin would have it.

Having clarified this, we do agree with Bennis \& Medin's more general concern that WEIRD samples may look particularly unusual on those measures that have been selected, developed, and honed for use in WEIRD populations. If Indian psychologists first identified a specific phenomenon that was of particular concern in India, they too might hone their methods to enhance their effects, resulting in Indian participants being outliers for that phenomenon. However, this is precisely the problem that the behavioral sciences face initial theories are largely derived from WEIRD samples, and, as such, we fail to consider phenomena that might be of greater concern elsewhere. As our target article emphasized, it remains an open question whether, when a full accounting is taken of all the psychological phenomena that exist throughout the world, WEIRD samples will remain any more unusual than other societies. At present, we lack the empirical data to evaluate this possibility, and hope that researchers strive to contribute to this database by identifying and studying phenomena that are more of a concern in other populations.

\section{R5. Does variability conceal psychological universals? Is WEIRD WRONG?}

Gaertner et al.'s commentary stands as the sole one that explicitly rejects our basic claim regarding populationlevel variability in psychological processes. Although this commentary is unique in its criticism of our key arguments, we suspect that many behavioral scientists share the intuitions underlying these commentators' critique, and we therefore devote substantial space to this in our response.

Gaertner et al. maintain that behavioral scientists study the culturally variant phenotypes of an underlying universal genotype. We note that this point is dependent on researchers being able to discern what the underlying universal psychological process is in the first place. But behavioral scientists do not have direct access to this underlying genotypic level; rather, we are in the business of studying questions at the phenotypic level, such as, whether people view themselves more positively than they are viewed by others, whether people succumb to the Müller-Lyer illusion, or at what point people reject low offers in the Ultimatum Game. The genotypic level is inferred on the basis of the phenotypic evidence; and when the phenotypic evidence comes from a narrow sample, such as North American undergraduates, researchers very well might incorrectly infer what is going on at the genotypic level. 
As an analogy, consider the case of understanding pregnancy sickness. All of the major hypotheses for why women get nausea and vomiting during their first trimester are evolutionary explanations. Some hypothesize that pregnancy sickness is an evolutionary byproduct of the body's need to alter hormonal levels, to permit the growth of a fetus. Other explanations propose that pregnancy sickness is an adaptation triggered by either threats from pathogens (in meat) or toxins (from plants). Which evolutionary explanation is correct? It turns out that key evidence comes from studies of small-scale societies that rely on very little meat and use corn as a staple (which does not have the toxins hypothesized to spark pregnancy sickness as a defense). These societies have plenty of pregnancy, but no pregnancy sickness, thus undermining the byproduct hypothesis (Fessler 2002; Flaxman \& Sherman 2000). So, assuming evolved universality does not preclude the need for comparative evidence to adjudicate among alternative hypotheses.

Now consider the self-serving attributional bias, which is operationalized as the tendency for people to take personal credit for their successes but to direct blame externally for their failures. An extensive meta-analysis revealed that the effect size for this bias is $d=1.05$ for Americans but $d=-0.30$ for Japanese (Mezulis et al. 2004): that is, the phenotypes are diametrically opposed. What is the underlying universal psychological process here? Just like the blind men trying to identify the elephant, one would reach an entirely different conclusion if one had Japanese data than if one had American data. The most complete view of the underlying beast requires multiple sources of evidence; the more diverse the populations studied, the better researchers will be able to triangulate on the underlying processes, be those universal psychological or ontogenetic processes.

The guiding assumption of much of the behavioral sciences has been that human behavior is the expression of universal underlying psychological processes. We submit that it is because of this assumption that the samples studied are as narrow as Arnett's (2008) analysis reveals. Indeed, there is little point to trek into the New Guinea highlands if highlanders share the same universal psychological processes as the undergraduates in a researcher's home university. In the target article we questioned whether this assumption is tenable - how does it stand up to the empirical evidence? For some domains (e.g., personality structure; sex differences in some mate preferences) the evidence for universality, at least at the level of functional universals (see Norenzayan \& Heine 2005), seems solid. In other domains (e.g., fairness motivations, moral principles, spatial perception), the high degree of variation among populations makes identifying an underlying universal psychological process more interesting (and more work). But we cannot address whether this assumption of universality is supported for a given hypothesized process until there is sufficient comparative data.

In some domains, it might be the case that there is not a common, universal psychological process. Many in the behavioral sciences have yet to take seriously the implications of epigenetic inheritance and culture-gene coevolution. A rising tide of evidence from epigenetics is showing how genetic systems modify gene expressions to adapt to local circumstances without altering the underlying DNA. This can create lasting heritable variation in individuals (epigenetic inheritance), and differences among populations, without any underlying differences in DNA base pair sequences - only differences in gene expression (Jablonka \& Raz 2009). Monozygotic twins diverge in their gene expression as they age because their epigenetic response systems modify their gene expressions (Fraga et al. 2005). In addition, potent forces of culture-gene coevolution could mean that different populations have genetically adapted to stable elements of their cultures, such as in the case of lactose tolerance (Laland et al. 2010). This does not mean that there are no basic principles to account for psychology and behavior; it just means that we must move one step back to principles from genetics, epigenetics, culturegene coevolution, and cultural evolution to explain human variation.

It seems to us that Gaertner et al. are offering an unfalsifiable hypothesis. They suggest that studying diverse populations will either yield evidence of similarities because of an underlying universal psychological process, or it will yield evidence of differences, which mask the underlying universal psychological process. They do not offer any means for discerning an underlying universal process in the face of population-level variability. Indeed, they do not seem willing to entertain alternative hypotheses that, for example, propose universal ontogenetic processes that give rise to different psychological processes under different conditions during development.

This is particularly evident in their discussion of population-level variability in self-enhancement motivations. Gaertner et al. challenge us by claiming that the crosscultural evidence regarding self-enhancement points to much universality. We tackle this claim at length here as it is central to their rejection of our thesis, and it is an ongoing controversy. ${ }^{3}$ First, they argue that this motivation is universal but expressed differently: Westerners enhance themselves in domains that are important to them (i.e., individualism), while East Asians enhance themselves in domains that are important to them (i.e., collectivism). This question has been investigated using a number of different methods. The results from the "better-thanaverage effect" largely support this hypothesis (Brown \& Kobayashi 2002; Sedikides et al. 2003). These are the only findings cited by Gaertner et al.

However, the other 11 methods that have addressed this same question (viz., the false-uniqueness bias, actual-ideal self-discrepancies, manipulations of success and failure, situation sampling, self-peer biases, relativelikelihood and absolute-likelihood optimism biases, open-ended self-descriptions, automatic self-evaluations, social relations model, and a corrected better-thanaverage effect) yield an opposite pattern of results - that is, East Asians do not self-enhance more in domains that are especially important to them (whereas Westerners do: Falk et al. 2009; Hamamura et al. 2007; Ross et al. 2005; Su \& Oishi 2010). A meta-analysis including all of the published studies on this topic finds no support for this hypothesis (Heine et al. 2007a; 2007b); the meta-analyses cited by Gaertner et al. (viz., Sedikides et al. 2005; 2007a) find different results because they excluded most of the studies that yielded contrary findings. Further, many research programs have documented that the better-than-average effect is a compromised measure of 
self-enhancement as it includes a number of cognitive biases that exaggerate estimates of self-enhancement (Chambers \& Windschitl 2004; Hamamura et al. 2007; Klar \& Giladi 1997; Krizan \& Suls 2008; Kruger 1999; Windschitl et al. 2008). That is, the sum total of the available evidence contradicts Gaertner et al.'s claim that East Asians are self-enhancing in domains of special importance to them.

A second argument for the universality of self-enhancement that Gaertner et al. offer is that the populationlevel variability is a function of different modesty norms such that the cultural differences disappear with nonexplicit measures. However, other studies of self-enhancement that employ hidden behavioral measures (which Gaertner et al. do not cite) find equally pronounced differences as those with explicit measures (Heine et al. 2000; 2001). Along these lines, Gaertner et al. cite evidence that cultural differences do not appear with the Implicit Association Test (IAT) measure of self-esteem (Greenwald \& Farnham 2000). This is the only method out of 31 that did not find a population-level difference in the magnitude of self-enhancement motivations between Westerners (average $d=0.87$ ) and East Asians (average $d=-0.01$ : Heine \& Hamamura 2007), yet none of the results from these 30 other methods are discussed by Gaertner et al. Further, the IAT measure of self-esteem has the least validity evidence of any of the IAT attitude measures (Hofmann et al. 2005), and this measure does not correlate reliably with other implicit measures of self-esteem, measures of explicit self-esteem, or other external validity criteria (Bosson et al. 2000; Falk et al. 2009). Hence, at present it is unclear what the self-esteem IAT measures, and it is noteworthy that it is the method that stands alone in not finding a difference in self-enhancement motivations between Westerners and East Asians.

Finally, Gaertner et al. argue that self-enhancement promotes adjustment equally in both Westerners and East Asians. But the relationship between self-enhancement and adjustment continues to be hotly debated, even among Western samples, with divergent results emerging depending on the methods used. In general, evidence for this is best with measures of the better-thanaverage effect and self-report measures of adjustment, where the individual answers items regarding both how positively people view themselves relative to others and how positively they view themselves with regards to their adjustment. The evidence is much weaker, and often contradictory, for studies that utilize objective benchmarks of self-enhancement (Colvin et al. 1995; Paulhus 1998; Robins \& Beer 2001; but see Taylor et al. 2003). Further, the only published study that measured selfenhancement and depression in both East Asian and Western locations finds a significantly weaker relation between the two constructs among Japanese than Canadians (Heine \& Lehman 1999). It is also worth noting that, although evidence for self-enhancement is far weaker among East Asians than Westerners, epidemiological studies find depression rates in East Asia to be approximately one-fifth that of North America (Kessler et al. 1994; Weissman et al. 1996) - a pattern that is difficult to explain if self-enhancement promotes well-being equally across populations.

In sum, Gaertner et al. claim that East Asians selfenhance similarly to North Americans, and that this reveals the universality of self-enhancement motivations. They are only able to make this claim by ignoring the vast majority of the relevant data. The assumption of underlying universal psychological processes is nothing more than that - an assumption, which needs to be evaluated against alternative hypotheses with empirical evidence from diverse human populations.

\section{R6. Misleading categories and contrasts?}

While agreeing with our major point, Astuti \& Bloch suggest that our series of telescoping contrasts, and especially our contrasts between small-scale and industrialized societies and between Western and non-Western societies, distorts or exaggerates the unusual nature of WEIRD people. They charge us with the "uncritical lumping together of a variety of disparate societies" and with using "under-theorized labels."

We agree with their concerns about uncritically lumping societies, which is why we sought to carefully avoid such pitfalls. To begin, in our introduction we wrote, "We emphasize that our presentation of telescoping contrasts is only a rhetorical approach guided by the nature of the available data. It should not be taken as capturing any unidimensional continuum, or suggesting any single theoretical explanation for the variation" (target article, sect. 1, para. 7). The first sentence in this quotation was meant to explain how our particular choices of telescoping contrasts were strictly driven by the lumpy and sparse distribution of the available data, not by any theorizing about the nature of the variation. The second sentence, regarding the unidimensional continuum, was meant to explicitly avoid any suggestion of what Astuti \& Bloch term a "unilineal path."

Next, because of our concern about lumping disparate societies, we displayed the data whenever possible. Figure 2 in our target article displays the 14 small-scale societies for the Müller-Lyer illusion, along with two industrialized populations. We also included all the samples of children on that figure. This allows the reader to draw his or her own conclusions, and highlights the degree of variation among small-scale societies. Figures $3 \mathrm{~A}, 3 \mathrm{~B}$, and $3 \mathrm{C}$ display the means for each of 15 smallscale societies on three different behavioral measures related to fairness. Again, this kind of graphical display was specifically intended to lay all the cards on the table, and avoid concealing population-level variation in broad categories. In the text we wrote,

For Dictator Game offers, Figure 3A shows that the U.S. sample has the highest mean offer, followed by the Sanquianga from Colombia, who are renowned for their prosociality (Kraul 2008). The U.S. offers are nearly double that of the Hadza, foragers from Tanzania, and the Tsimane, forager-horticulturalists from the Bolivian Amazon. ... [F] or Ultimatum Game offers, the United States has the second highest mean offer, behind the Sursurunga from Papua New Guinea. (target article, sect. 3.2, para. 5)

How is this lumping the small-scale societies together? Figure 4 from our target articles displays the available data from nine non-Western and seven Western societies for both the punishment of free-riding and for antisocial punishment. The reader can see the interesting variation within both Western and non-Western societies, 
and can even apply his or her own categorization schemes. In Figure 5, we show the percentage difference between analytic and holistic judgments for six different samples, which we compiled ourselves from different sources in order to illustrate the variation as accurately as possible.

Astuti \& Bloch express forceful charges against our efforts, while agreeing with our main point. Given this, we believe it would have been more constructive to have explained how they would have presented the data and made the case more effectively.

\section{R7. Explaining the variation: Why are WEIRD samples so unusual?}

For the purposes of our target article we remained largely agnostic regarding explanations for the peculiar nature of WEIRD psychology, although we did point out that this should not be unexpected, given the rather odd ways of life of most WEIRD people, especially compared to those of small-scale societies and the environments of ancestral humans. Contra Danks \& Rose, we did not mean to suggest that this variation is fundamentally inexplicable, or that we should not try to explain it. In fact, all three of us (Henrich, Heine, and Norenzayan) have been engaged in trying to explain various elements of it for much of our careers.

Several commentaries, when viewed together, suggest two proximate explanations for why WEIRD subjects are often unusual. First, following Majid \& Levinson, the English language apparently occupies an obscure corner of the design space of possible languages, potentially giving theorists misleading points of departure or unusual folk intuitions. Majid \& Levinson worry that this "English-bias" may be impacting theorizing in the cognitive sciences, while Machery and Stich show that it has impacted philosophical inquiry. By citations, the top four sources of research in psychology are all from Englishspeaking countries (see May 1997): (1) the United States, (2) the United Kingdom, (3) Canada, and (4) Australia (for comparison, the top sources of research in physics are [1] the United States, [2] Germany, [3] Japan, and [4] France).

The second explanation combines arguments and evidence offered by Lancy, Fernald, and Karasik et al., suggesting at least the proximate end of a theory that may illuminate a wide range of cognitive difference between WEIRD populations and others. Lancy lays the groundwork by highlighting the relative strangeness, in a broad global and historical context, of modern middleand upper-class American beliefs, values, cultural models, and practices vis-à-vis childrearing. Fernald and Karasik et al. review evidence that is beginning to document how these practices impact cognitive, linguistic, and motor development, including long-term cognitive outcomes.

At a more ultimate level, we speculate that in the context of mobile, meritocratic societies like those of the United States, Western Europe, and Australia, cultural evolutionary processes rooted in our evolved tendencies to imitate successful and prestigious individuals (Henrich \& Gil-White 2001) will favor the spread of child-rearing traits that speed up and enhance the development of those particular cognitive and social skills that eventually translate into social and economic success in these populations. This kind of cultural evolutionary process may be part of what is driving the dramatic increases in IQ observed in many industrialized nations over the last century (Flynn 2007), along with increases in biases toward analytical reasoning and individualism. It would also explain the obsession with active instruction of all kinds shown by middle- and upper-class Americans (Lancy 2008).

In our target article we wanted to avoid making any theoretical claims regarding the origins of the psychological differences we highlighted. We suggested that WEIRD psychology probably arises from a myriad of different proximate causal sources that, at best, have been aggregated in WEIRD populations. The two hypotheses mentioned above illustrate this. It is likely a coincidence that the first population to seriously engage in the systematic study of psychology and decision-making happened to speak English. However, it is probably not a coincidence that the economic system of this population happened to favor certain child-rearing practices and ways of reasoning. Overall, we suspect that many of the phenomena for which WEIRD samples occupy extreme positions do so for quite distinct causal reasons, including some researcher-created biases. Once the behavioral sciences accept the existence or potential existence - of broad-ranging variation among populations, we can commence with the more interesting endeavor of explaining that variation at both proximate and ultimate levels of inquiry.

\section{R8. Closing words}

We have a vision for the future of scientific efforts to understand the foundations of human psychology and behavior. Research programs need to increasingly emphasize large-scale, highly interdisciplinary, fully international research networks that maintain long-term, ongoing, research projects among diverse populations that collect data over the full life cycle using an integrated set of methodological tools, including wide-ranging experimental techniques, quantitative and qualitative ethnography, surveys, brain imaging, and biomarkers. Questions and methods are best devised and designed at collaborative meetings of these international research networks.

\section{NOTES}

1. Data from http://www.internetworldstats.com.

2. Machery asks, "Why are most examples in Henrich et al.'s article drawn from social psychology? ... Would we find the same cross-cultural variation if we focused on the phenomena discussed in perceptual and cognitive psychology textbooks?" Our target article reviews findings from four visual illusions, induction, categorization, memory, attention, spatial cognition, prospect theory, decision biases, perceptual biases, IQ, and spatial abilities. These are all foci in cognitive psychology texts. Several commentaries and our response review evidence/findings on underwater vision, motor development (Karasik et al.), cognitive development (Fernald), and neural activation in the amygdala (Chiao \& Cheon). These are not domains of social psychology.

3. Readers interested in the controversy can see Brown and Kobayashi (2002), Heine (2005), Heine and Hamamura (2007), Heine et al. (2007a; 2007b), and Sedikides et al. (2005; 2007a; 2007b). 
References/Henrich et al.: The weirdest people in the world?

4. Astuti \& Bloch suggest that "small-scale" is a euphemism for "primitive." The discrete label small-scale societies is a descriptive term, meant to distinguish social groups living in small, geographically distinct populations (e.g., villages) that range in size from a handful to a few thousand. Prototypically, the social organization of these groups is local, and often kin-based. The division of labor is not extensive, and households typically produce a substantial fraction of their own food. Interactions are mostly face-to-face. Of course, all these dimensions are continuous, so this label, like many, is meant to roughly distinguish one region of an $n$-dimensional space. The term is regularly used by anthropologists in the 21st century (Borgerhoff Mulder et al. 2009; Walker et al. 2006) without carrying any sense of mental or physical "primitiveness."

\section{References}

[The letters " $a$ " and "r" before author's initials stand for target article and response references, respectively]

Abarbanell, L. \& Hauser, M. (in press) Mayan morality: An exploration of permissible harms. Cognition. [SS]

Adams, G. (2005) The cultural grounding of personal relationships: Enemyship on West African worlds. Journal of Personality and Social Psychology 88:94868. $[\mathrm{aJH}]$

Adolph, K. E., Karasik, L. B. \& Tamis-LeMonda, C. S. (2010) Motor skills. In: Handbook of cultural developmental science, ed. M. H. Bornstein, pp. 61-88. Taylor \& Francis. [LBK]

Allik, J. \& McCrae, R. R. (2004) Toward a geography of personality traits: Patterns of profiles across 36 cultures. Journal of Cross-Cultural Psychology 35:1328. [aJH]

Al-Shehab, A. J. (2002) A cross-sectional examination of levels of moral reasoning in a sample of Kuwait University faculty members. Social Behavior and Personality 30:813-20. [aJH]

Alvard, M. (2004) The Ultimatum Game, fairness, and cooperation among big game hunters. In: Foundations of human sociality: Economic experiments and ethnographic evidence from fifteen small-scale societies, ed. J. Henrich, R. Boyd, S. Bowles, C. Camerer, E. Fehr \& H. Gintis, pp. 413-35. Oxford University Press. $[\mathrm{rJH}]$

Anderson, S. L., Adams, G. \& Plaut, V. C. (2008) The cultural grounding of personal relationship: The importance of attractiveness in everyday life. Journal of Personality and Social Psychology 95:352-68. [aJH]

Arnett, J. (2008) The neglected 95\%: Why American psychology needs to become less American. American Psychologist 63(7):602-14. [JYC, AF, arJH, PRoc]

Asch, S. E. (1951) Effects of group pressure upon the modification and distortion of judgments. In: Groups, leadership and men, ed. H. Guetzkow, pp. 177-90. Carnegie. $\quad[\mathrm{a} J \mathrm{H}]$

Asch, S. E. (1952) Group forces in the modification and distortion of judgments. In: Social Psychology, pp. 450-501. Prentice Hall. [aJH]

Astuti, R. (2001) Are we all natural dualists? A cognitive developmental approach. Journal of the Royal Anthropological Institute 7(3):429-47. [aJH]

Astuti, R. \& Harris, P. L. (2008) Understanding mortality and the life of the ancestors in Madagascar. Cognitive Science 32:713-40. [RA]

Astuti, R., Solomon, G. E. A. \& Carey, S. (2004) Constraints on conceptual development. A case study of the acquisition of folkbiological and folksociological knowledge in Madagascar. Monographs of the Society for Research in Child Development 69, Serial No. 277; 69(3):vii-135. [RA, aJH]

Atran, S. (1993) Ethnobiological classification: Principles of categorization of plants and animals in traditional societies - Berlin, B. Current Anthropology 34(2):195-98. [aJH]

Atran, S. (2005) Adaptationism for human cognition: Strong, spurious or weak? Mind and Language 20(1):39-67. [aJH]

Atran, S. \& Medin, D. L. (2008) The native mind and the cultural construction of nature. MIT Press. [rJH]

Atran, S., Medin, D. L., Lynch, E., Vapnarsky, V., Ucan, E. E. \& Sousa, P. (2001) Folkbiology doesn't come from folkpsychology: Evidence from Yukatek Maya in cross-cultural perspective. Journal of Cognition and Culture 1(1):3-42. $[\mathrm{aJH}]$

Atran, S., Medin, D. L. \& Ross, N. (2005) The cultural mind: Environmental decision making and cultural modeling within and across populations. Psychological Review 112(4):744-76. [arJH]

Atran, S., Medin, D. L., Ross, N., Lynch, E., Coley, J. D., Ek, E. U. \& Vapnarsky, V. (1999) Folkecology and commons management in the Maya Lowlands.
Proceedings of the National Academy of Sciences USA 96(13):7598-603. $[\mathrm{rJH}]$

Baek, H. (2002) A comparative study of moral development of Korean and British children. Journal of Moral Education 31:373-91. [aJH]

Bahry, D. L. \& Wilson, R. K. (2006) Confusion or fairness in the field? Rejection in the Ultimatum Game under the strategy method. Journal of Economic Behavior and Organization 60(1):37-54. [aJH]

Baillargeon, R. (1994) How do infants learn about the physical world? Current Directions in Psychological Science 3:133-40. [AMaj]

Bakewell, M., Shi, P. \& Zhang, J. Z. (2007) More genes underwent positive selection in chimpanzee evolution than in human evolution. Proceedings of the National Academy of Sciences USA 104:7489-94. [AMar]

Baldry, J. C. (1986) Tax evasion is not a gamble: A report on two experiments. Economics Letters 22(4):333-35. [SJC]

Bandura, A. (1982) Self-efficacy mechanism in human agency. American Psychologist 37:122-47. [aJH]

Bang, M., Medin, D. L. \& Atran, S. (2007) Cultural mosaics and mental models of nature. Proceedings of the National Academy of Sciences USA 104:1386874. $[\operatorname{arJH}]$

Baran, N., Sapienza, P. \& Zingales, L. (2009) Can we infer social preferences from the lab? Evidence from the Trust Game. Unpublished manuscript, University of Chicago. [rJH]

Bard, K. A. (1998) Social-experiential contributions to imitation and emotion in chimpanzees. In: Intersubjective communication and emotion in early ontogeny, ed. S. Bråten, pp. 208-27. Cambridge University Press. [DAL]

Bard, K. A. (2008) Development of emotional expression in chimpanzee and human infants (in English with Russian translation). Psychological Bulletin of Novosibirsk State University, Russia 2(2):98-105. [DAL]

Bard, K. A. \& Leavens, D. A. (2009) Socio-emotional factors in the development of joint attention in human and ape infants. In: Learning from animals? Examining the nature of human uniqueness, ed. L. S. Röska-Hardy \& E. M. Neumann-Held, pp. 89-104. Psychology Press. [DAL]

Bardsley, N., Cubitt, R., Loomes, G., Moffatt, P., Starmer, C. \& Sugden, R. (2010) Experimental economics: Rethinking the rules. Princeton University Press. [SG]

Barrett, H. C. (2005) Adaptations to predators and prey. In: The handbook of evolutionary psychology, ed. D. M. Buss, pp. 200-23. Wiley. [EM]

Barrett, H. C. (2006) Modularity and design reincarnation. In: The innate mind: Culture and cognition, ed. P. Carruthers, S. Laurence \& S. Stich, pp. 199-217. Oxford University Press. [aJH]

Barrett, H. C. \& Behne, T. (2005) Children's understanding of death as the cessation of agency: A test using sleep versus death. Cognition 96(2):93-108. $[\operatorname{arj} \mathrm{H}]$

Bastien, G. J., Schepens, B., Willems, P. A. \& Heglund, N. C. (2005) Energetics of load carrying in Nepalese porters. Science 308:1755. [LBK]

Baumrind, D. (1971) Current patterns of parental authority. Developmental Psychology Monographs 4(1, pt. 2):1-103. [DFL]

Bayley, N. (1969) Bayley scales of infant development. The Psychological Corporation. [LBK]

Becker, E. (1973) The denial of death. Free Press. [aJH]

Beja-Pereira, A., Luikart, G., England, P. R., Bradley, D. G., Jann, O. C., Bertorelle, G., Chamberlain, A. T., Nunes, T. P., Metodiev, S., Ferrand, N. \& Erhardt, G. (2003) Gene-culture coevolution between cattle milk protein genes and human lactase genes. Nature Genetics 35(4):311-13. [aJH]

Bellemare, C. \& Kröger, S. (2007) On representative social capital. European Economic Review 51:183-202. [aJH]

Bellemare, C., Kröger, S. \& Van Soest, A. (2008) Measuring inequity aversion in a heterogeneous population using experimental decisions and subjective probabilities. Econometrica 76(4):815-39. [SG, aJH]

Belsky, J., Steinberg, L. \& Draper, P. (1991) Childhood experience, interpersonal development, and reproductive strategy: An evolutionary theory of socialization. Child Development 62:647-70. [KP]

Benet-Martinez, V. \& Waller, N. G. (1995) The Big Seven factor model of personality description: Evidence for its cross-cultural generality in a Spanish sample. Journal of Personality and Social Psychology 69:701-18. [aJH]

Bennett, W. C. \& Zingg, R. M. (1935) The Tarahumara, an Indian tribe of northern Mexico. University of Chicago Press. [LBK]

Berlin, B. (1992) Ethnobiological classification: Principles of categorization of plants and animals in traditional societies. Princeton University Press. [aJH]

Berlin, B. \& Kay, P. (1969) Basic color terms: Their universality and evolution. University of California Press. [AMaj]

Berry, J. W. (1966) Emne and Eskimo perceptual skills. International Journal of Psychology 1:207-29. [aJH]

Billing, J. \& Sherman, P. W. (1998) Antimicrobial functions of spices: Why some like it hot. Quarterly Review of Biology 73(1):3-49. [aJH]

Bloch, M. (2005) Where did anthropology go? Or the need for "human nature." In: Essays on cultural transmission, ed. M. Bloch, pp. 1-19. Berg. [RA] 
Boesch, C. (2007) What makes us human (Homo sapiens)? The challenge of cognitive cross-species comparison. Journal of Comparative Psychology 121(3):227-40. [CB, aJH, DAL]

Boesch, C. (2008) Taking development and ecology seriously when comparing cognition. Journal of Comparative Psychology 122(4):453-55. [CB]

Boesch, C. (2009) The real chimpanzee: Sex strategies in the forest. Cambridge University Press. [CB]

Boesch, C. \& Boesch, H. (1984) Mental map in wild chimpanzees: An analysis of hammer transports for nut cracking. Primates 25:160-70. [CB]

Boesch, C. \& Boesch, H. (1989) Hunting behavior of wild chimpanzees in the Taï National Park. American Journal of Physical Anthropology 78:547-73. [CB]

Boesch, C., Crockford, C., Herbinger, I., Wittig, R., Moebius, Y. \& Normand, E. (2008) Intergroup conflicts among chimpanzees in Taï National Park: Lethal violence and the female perspective. American Journal of Primatology 70:114. $[\mathrm{CB}]$

Boesch, C., Eckhard, N., Bolé, C. \& Boesch, H. (2010) Altruism in forest chimpanzees: The case of adoption. PLoS One 5(1):e8901. [CB]

Boesch, C., Head, J. \& Robbins, M. (2009) Complex tool sets for honey extraction among chimpanzees in Loango National Park, Gabon. Journal of Human Evolution 56:560-69. [CB]

Bohnet, I., Greig, F., Herrmann, B. \& Zeckhauser, R. (2008) Betrayal aversion. Evidence from Brazil, China, Oman, Switzerland, Turkey, and the United States. American Economic Review 98(1):294-310. [SG]

Bolyanatz, A. H. (under review) Sursurunga Dictator and Ultimatum Game results. Submission for: Experimenting with social norms: Fairness and punishment in cross-cultural perspective, ed. J. Henrich \& J. Ensminger. [rJH]

Bond, R. \& Smith, P. B. (1996) Culture and conformity: A meta-analysis of studies using Asch's (1952b, 1956) line judgment task. Psychological Bulletin 119(1):111-37. [aJH]

Borgerhoff Mulder, M., Bowles, S., Hertz, T., Bell, A., Beise, J., Clark, G., Fazzio, I., Gurven, M., Hill, K., Hooper, P. L., Irons, W., Kaplan, H., Leonetti, D., Low, B., Marlowe, F., McElreath, R., Naidu, S., Nolin, D., Piraino, P., Quinlan, R., Schniter, E., Sear, R., Shenk, M., Smith, E. A., von Rueden, C. \& Wiessner, P. (2009) Intergenerational wealth transmission and the dynamics of inequality in small-scale societies. Science 326(5953):682-88. [rJH]

Bornstein, M. H. (1980) Cross-cultural developmental psychology. In: Comparative methods in psychology, ed. M. H. Bornstein, pp. 231-81. Erlbaum. [LBK]

Bornstein, M. H. (1995) Form and function: Implications for studies of culture and human development. Culture and Psychology 1:123-37. [LBK]

Bosson, J. K., Swann, W. B. \& Pennebaker, J. W. (2000) Stalking the perfect measure of implicit self-esteem: The blind men and the elephant revisited? Journal of Personality and Social Psychology 79(4):631-43. [rJH]

Bouchard, T. J. (2004) Genetic influence on human psychological traits - A survey. Current Directions in Psychological Science 13(4):148-51. [ajH]

Bowerman, M. (1996) Learning how to structure space for language: A cross-linguistic perspective. In: Language and space, ed. P. Bloom, M. A. Peterson, L. Nadel \& M. F. Garrett, pp. 385-436. MIT Press. [AMaj]

Bowerman, M. \& Choi, S. (2001) Shaping meanings for language: Universal and language-specific in the acquisition of spatial semantic categories. In: Language acquisition and conceptual development, ed. M. Bowerman \& S. C. Levinson, pp. 475-511. Cambridge University Press. [AMaj]

Boyd, R. \& Richerson, P. J. (1985) Culture and the evolutionary process. University of Chicago Press. [ajH]

Bramble, D. M. \& Lieberman, D. E. (2004) Endurance running and the evolution of Homo. Nature 432(7015):345-52. [rJH]

Brandt, R. (1954) Hopi ethics: A theoretical analysis. University of Chicago Press. [SS]

Bril, B. (1988) Bain et gymnastique neonatale: Enfants Bambara du Mali (Motion Picture). France: Centre d'Etude des Processus Cognitifs et du Langage Ecole des Hautes Etudes en Sciences Socieles. [LBK]

Brown, D. E. (1991) Human universals. McGraw-Hill. [aJH]

Brown, J. D. (in press) Across the (not so) great divide: Cultural similarities in selfesteem evaluative processes. Social and Personality Psychology Compass. [LG]

Brown, J. D., Cai, H., Oakes, M. A. \& Deng, C. (2009) Cultural similarities in selfesteem functioning: East is East and West is West, but sometimes the twain do meet. Journal of Cross-Cultural Psychology 40:140-57. [LG]

Brown, J. D. \& Kobayashi, C. (2002) Self-enhancement in Japan and America. Asian Journal of Social Psychology 5:145-68. [LG, rJH]

Brown, R. (1986) Social psychology, 2nd edition. Free Press. [aJH]

Bucciarelli, M. \& Johnson-Laird, P. N. (1999) Strategies in syllogistic reasoning. Cognitive Science 23:247-303. [SSK]

Bucciarelli, M. \& Johnson-Laird, P. N. (2005) Naïve deontics: A theory of meaning, representation, and reasoning. Cognitive Psychology 50:159-93. [SSK]

Bucciarelli, M., Khemlani, S. \& Johnson-Laird, P. N. (2008) The psychology of moral reasoning. Judgment and Decision Making 3:121-39. [SSK]
Buchtel, E. \& Norenzayan, A. (2008) Which should you use, intuition or logic? Cultural differences in injunctive norms about reasoning. Asian Journal of Social Psychology 11:264-73. [arJH]

Bulloch, M. J., Boysen, S. T. \& Furlong, E. E. (2008) Visual attention and its relation to knowledge states in chimpanzees, Pan troglodytes. Animal Behaviour $76: 1147-55$. [DAL]

Burenhult, N. (2006) Body part terms in Jahai. Language Sciences 28:162-80. [AMaj]

Burke, B., Martens, A. \& Faucher, E. (2010) Two decades of terror management theory: A meta-analysis of mortality salience research. Personality and Social Psychological Review 14:155-95. [aJH]

Burnham, T. C. \& Johnson, D. D. (2005) The biological and evolutionary logic of human cooperation. Analyse and Kritik 27:113-35. [rJH]

Burton, R. \& Whiting, J. (1961) The absent father and cross-sex identity. MerrillPalmer Quarterly 7(85-95):85. [aJH]

Buss, D. M. (1989) Sex-differences in human mate preferences: Evolutionary hypothesis tested in 37 cultures. Behavioral and Brain Sciences 12(1):1-14. $[\mathrm{aJH}]$

Butterworth, G. (2003) Pointing is the royal road to language for babies. In: Pointing: Where language, culture, and cognition meet, ed. S. Kita, pp. 9-33. Erlbaum. [DAL]

Cai, H., Brown, J. D., Deng, C. \& Oakes, M. A. (2007) Self-esteem and culture: Differences in cognitive self-evaluations or affective self-regard? Asian Journal of Social Psychology 10:162-70. [LG]

Cai, H., Wu, Q. \& Brown, J. D. (2009) Is self-esteem a universal need? Evidence from the People's Republic of China. Asian Journal of Social Psychology 12:104-20. [LG]

Callaghan, T., Rochat, P., Lillard, A., Claux, M. L., Odden, H., Itakura, S., Tapanya, S. \& Singh, S. (2005) Synchrony in the onset of mental-state reasoning Evidence from five cultures. Psychological Science 16(5):378-84. [aJH]

Camerer, C. (2003) Behavior game theory: Experiments in strategic interaction. Princeton University Press. [ajH]

Campbell, D. T. (1996) Can we overcome worldview incommensurability/relativity in trying to understand the other? In: Ethnography and human development: Context and meaning in social inquiry, ed. R. Jessor, A. Colby \& R. A. Shweder, pp. 153-72. University of Chicago Press. [RAS]

Campbell, D. T. \& Stanley, J. C. (1963) Experimental and quasi-experimental designs for research. Rand McNally. [VJK]

Cappelen, A. W., Hole, A. D., Sorensen, E. O. \& Tungodden, B. (2007) The pluralism of fairness ideals: An experimental approach. American Economic Review 97(3):818-27. [NB]

Cardenas, J. C. \& Carpenter, J. (2008) Behavioural development economics: Lessons from field labs in the developing world. Journal of Development Studies 44(3):311-38. [aJH]

Carey, S. (1985) Conceptual change in childhood. MIT Press. [aJH]

Carey, S. (1995) On the origins of causal understanding. In: Causal cognition, ed. D. Sperber, D. Premack \& A. Premack, pp. 268-302. Oxford University Press. [aJH]

Carpendale, J. \& Lewis, C. (2004) Constructing an understanding of mind: The development of children's social understanding within social interaction. Behavioral and Brain Sciences 27:79-151. [CB]

Carpenter, J., Burks, S. \& Verhoogen, E. (2005) Comparing students to workers: The effects of social framing on behavior in distribution games. In: Field experiments in economics, ed. J. Carpenter, G. W. Harrison \& J. A. List, pp. 261-90. JAI Press/Elsevier. [aJH]

Carpenter, J., Connolly, C. \& Myers, C. K. (2008) Altruistic behavior in a representative dictator experiment. Experimental Economics 11(3):282-98. [SG, aJH]

Carpenter, M., Tomasello, M. \& Savage-Rumbaugh, S. (1995) Joint attention and imitative learning in children, chimpanzees, and enculturated chimpanzees. Social Development 4:217-37. [DAL]

Carroll, S. P. (1993) Divergence in male mating tactics between two populations of the soapberry bug: I. Guarding versus nonguarding. Behavioral Ecology 4:156-64. [KP]

Carroll, S. P. \& Corneli, P. S. (1995) Divergence in male mating tactics between two populations of the soapberry bug: II. Genetic change and the evolution of a plastic reaction norm in a variable social environment. Behavioral Ecology 6:46-56. [KP]

Carter, J. R. \& Irons, M. D. (1991) Are economists different, and if so, why? Journal of Economic Perspectives 5(Spring):171-77. [aJH]

Carver, C. S. (2004) Editorial. Journal of Personality and Social Psychology 86:95. [ajH]

Cavalli-Sforza, L. L., Menozzi, P. \& Piazza, A. (1994) The history and geography of human genes. Princeton University Press. [aJH]

Ceci, S. J. (1996) On intelligence: A bio-ecological treatise on intellectual development, 2nd edition. Harvard University Press. [SJC]

Ceci, S. J., Fitneva, S. A. \& Williams, W. M. (2010) Representational constraints on the development of memory and metamemory: A developmental-representational theory. Psychological Review 117:464-95. [SJC] 
References/Henrich et al.: The weirdest people in the world?

Chambers, J. R. \& Windschitl, P. D. (2004) Biases in social comparative judgments: The role of nonmotivated factors in above-average and comparative-optimism effects. Psychological Bulletin 130:813-38. [rJH]

Chen, C., Lee, S.-Y. \& Stevenson, H. W. (1995) Response style and cross-cultural comparisons of rating scales amongst East Asian and North American students Psychological Science 6:170-75. [aJH]

Cheung, F. M., Leung, K., Fan, R. M., Song, W., Zhang, J. X. \& Zhang, J. P. (1996) Development of the Chinese Personality Assessment Inventory. Journal of Cross-Cultural Psychology 27:181-99. [aJH]

Chew, S. (2005) Double binds around my feet: The enormity of the everyday in women's writing and writing about women. Journal of Gender Studies 14:13746. $[\mathrm{LBK}]$

Chiao, J. Y. (2009) Cultural neuroscience: A once and future discipline. Progress in Brain Research 178:287-304. [JYC]

Chiao, J. Y., Iidaka, T., Gordon, H. L., Nogawa, J., Bar, M., Aminoff, E., Sadato, N. \& Ambady, N. (2008) Cultural specificity in amygdala response to fear faces. Journal of Cognitive Neuroscience 20(12):2167-74. [JYC]

Choi, I. \& Nisbett, R. E. (1998) Situational salience and cultural differences in correspondence bias and the actor-observer bias. Personality and Social Psychology Bulletin 24:949-60. [arJH]

Choi, I., Nisbett, R. E. \& Norenzayan, A. (1999) Causal attribution across cultures: Variation and universality. Psychological Bulletin 125(1):47-61. [aJH]

Chua, H. F., Boland, J. E. \& Nisbett, R. E. (2005) Cultural variation in eye movements during scene perception. Proceedings of the National Academy of Sciences USA 102:12629-33. [aJH]

Church, A. T., Katigbak, M. S., Del Prado, A. M., Ortiz, F. A., Mastor, K. A., Harumi, Y., Tanaka-Matsumi, J., De Jesús Vargas-Flores, J., Ibáñez-Reyes, J., White, F. A., Miramontes, L. G., Reyes, J. A. S. \& Cabrera, H. F. (2006) Implicit theories and self-perceptions of traitedness across cultures: Toward integration of cultural and trait psychology perspectives. Journal of CrossCultural Psychology 37(6):694-716. [aJH]

Cialdini, R. (2009) We have to break up. Perspectives on Psychological Science 4:56. [TSR]

Cialdini, R. B., Borden, R. J., Thorne, A., Walker, M. R., Freeman, S. \& Slone, L. R. (1976) Basking in reflected glory: Three (football) field studies. Journal of Personality and Social Psychology 34:366-75. [SK]

Clark, H. H. (1973) Space, time, semantics, and the child. In: Cognitive development and the acquisition of language, ed. T. Moore, pp. 27-63. Academic Press. [AMaj]

Coe, C. L. \& Lubach, G. R. (2008) Fetal programming: Prenatal origins of health and illness. Current Directions in Psychology 17:36-41. [AF]

Cohen, D., Hoshino-Browne, E. \& Leung, A. (2007) Culture and the structure of personal experience: Insider and outsider phenomenologies of the self and social world. In: Advances in experimental social psychology, vol. 39, ed. M. P. Zanna, pp. 1-67. Academic Press. [aJH]

Cohen, E. (2007) The mind possessed: The cognition of spirit possession in an AfroBrazilian religious tradition. Oxford University Press. [rJH]

Cole, M. (1996) Cultural psychology: A once and future discipline. Harvard University Press. [RAS]

Cole, M., Gay, J., Glick, J. A., Sharp, D. W., Ciborowski, T., Frankel, F., Kellemu, J. \& Lancy, D. F. (1971) The cultural context of learning and thinking. Basic Books. [DFL]

Colvin, C. R., Block, J. \& Funder, D. C. (1995) Overly positive self-evaluations and personality: Negative implications for mental health. Journal of Personality and Social Psychology 68:1152-62. [rJH]

Corbett, G. G. \& Davies, I. R. L. (1995) Linguistic and behavioural measures for ranking basic colour terms. Studies in Language 19:301-57. [AMaj]

Cronk, L. (2007) The influence of cultural framing on play in the trust game: A Maasai example. Evolution and Human Behavior 28(5):352-58. [NB, EM]

Croson, R. \& Gächter, S. (2010) The science of experimental economics. Journal of Economic Behavior and Organization 73(1):122-31. [SG]

Cross, P. (1977) Not can but will college teaching be improved. New Directions for Higher Education 17:1-15. [aJH]

Csibra, G. \& Gergely, G. (2009) Natural pedagogy. Trends in Cognitive Sciences 13(4):148-53. [DFL, KP]

Daly, M. \& Wilson, M. (1988) Homicide. Aldine de Gruyter. [aJH]

D’Andrade, R. G. (1995) The development of cognitive anthropology. Cambridge University Press. [aJH]

Darwin, C. (1872) The expression of the emotions in man and animals. J. Murray. [DMTF]

Dasen, P. R., Inhelder, B., Lavalée, M. \& Retschitzki, J. (1978) Naissance de l'Intelligence Chez Infant Baoulè de Côte d'Ivoire. Hans Huber. [DFL]

Dasen, P. R., Mishra, R. C., Niraula, S. \& Wassmann, J. (2006) Développement du langage et de la cognition spatiale géocentrique. Enfance 58:146-58. [aJH]

Davidoff, J., Davies, I. \& Roberson, D. (1999) Colour categories in a stone-age tribe. Nature 398:203-04. [AMaj]

Davis, B. E., Moon, R. Y., Sachs, H. C. \& Ottolini, M. C. (1998) Effects of sleep position on infant motor development. Pediatrics 102:1135-40. [LBK]
Dawkins, R. (2006) The God delusion. Houghton Mifflin. $\quad[\mathrm{rJH}]$

Degos, J. D. (2001) Pointing disorders. Revue de Neuropsychologie 11:257-65. [DAL]

Dehaene, S. (1997) The number sense: How the mind creates mathematics. Oxford University Press. [arjH]

de Tocqueville, A. (1835) Democracy in America, trans. G. Lawrence. University of Chicago Press. [aJH]

Deutsch, M., Carroll, C., Sytsma, J. \& Machery, E. (2010) Cross-cultural semantics and the speaker's/semantic reference distinction. Unpublished manuscript, University of Pittsburgh. [EM, SS]

de Waal, F. B. M. (1982) Chimpanzee politics: Power and sex among apes. Harper and Row. [DAL]

de Waal, F. B. M. (2001) The ape and the sushi master: Cultural reflections of a primatologist. Basic Books. [CB]

Diener, E., Diener, M. \& Diener, C. (1995) Factors predicting the subjective wellbeing of nations. Journal of Personality and Social Psychology 69:815-64. [aJH]

Dryer, M. S. (2008) Polar questions. In: The world atlas of language structures online, ed. M. Haspelmath, M. S. Dryer, D. Gil \& B. Comrie. Max Planck Digital Library, Ch. 116. Available at: http://wals.info/feature/116. Accessed 17 December 2009. [AMaj]

Eagly, A. H. \& Wood, W. (1999) The origins of sex differences in human behavior: Evolved dispositions versus social roles. American Psychologist 54:408-423. $[\mathrm{aJH}]$

Earley, P. C. (1993) East meets West meets Mideast: Further explorations of collectivistic and individualistic work groups. Academy of Management Journal 36:319-48. [aJH]

Ebbesen, E. B. \& Konečni, V. J. (1975) Decision making and information integration in the courts: The setting of bail. Journal of Personality and Social Psychology 32:805-21. [VJK]

Ebbesen, E. B., Parker, S. \& Konečni, V. J. (1977) Laboratory and field analyses of decisions involving risk. Journal of Experimental Psychology: Human Perception and Performance 3:576-89. [VJK]

Edgerton, R. B. (1971) The individual in cultural adaptation: A study of four East African peoples. University of California Press. $[\mathrm{r} J \mathrm{H}]$

Egas, M. \& Riedl, A. (2008) The economics of altruistic punishment and the maintenance of cooperation. Proceedings of the Royal Society of London $B$ - Biological Sciences 275(1637):871-78. [aJH]

Eibl-Eibesfeldt, I. (2010) Human ethology. Aldine de Gruyter. [DAL]

Ekman, P. (1999a) Basic emotions. In: The handbook of cognition and emotion, ed. T. Dalgleish \& T. Power, pp. 45-60. Wiley. [aJH]

Ekman, P. (1999b) Facial expressions. In: The handbook of cognition and emotion, ed. T. Dalgleish \& T. Power, pp. 301-20. Wiley. [aJH]

Ekman, P. \& Friesen, W. V. (1971) Constants across cultures in the face and emotion. Journal of Personality and Social Psychology 17(2):124-29. [DMTF]

Elfenbein, H. A. \& Ambady, N. (2002) On the universality and cultural specificity of emotion recognition: A meta-analysis. Psychological Bulletin 128:203-35. [aJH]

Elliot, I. T., Chirkov, V. I., Kim, Y. \& Sheldon, K. M. (2001) A cross-cultural analysis of avoidance (relative to approach) personal goals. Psychological Science 12:505-10. [aJH]

Ellis, B. J., McFadyen-Ketchum, S., Dodge, K. A., Pettit, G. S. \& Bates J. E. (1999) Quality of early family relationships and individual differences in the timing of pubertal maturation in girls: A longitudinal test of an evolutionary model. Journal of Personality and Social Psychology 77:387-401. [KP]

Enfield, N. J. (2001) "Lip-pointing": A discussion of form and function with reference to data from Laos. Gesture 1:185-212. [DAL]

Ensminger, J. (2002) Experimental economics: A powerful new method for theory testing in anthropology. Theory in economic anthropology, pp. 59-78. AltaMira Press. [NB]

Ensminger, J. (2004) Market integration and fairness: Evidence from Ultimatum, Dictator and Public Goods experiments in East Africa. In: Foundations of human sociality: Economic experiments and ethnographic evidence from fifteen small-scale societies, ed. J. Henrich, R. Boyd, S. Bowles, C. Camerer, E. Fehr \& H. Gintis, pp. 356-81. Oxford University Press. $[\mathrm{rJH}]$

Ensminger, J. \& Cook, K. (under review) Pro-social norms among rural and urban small communities in America: Evidence from dictator, ultimatum, and public goods games. In: Experimenting with social norms: Fairness and punishment in cross-cultural perspective, ed. J. Ensminger \& J. Henrich. Russell Sage Foundation Press. [aJH]

Evans, N. \& Levinson, S. C. (2009) The myth of language universals: Language diversity and its importance for cognitive science. Behavioral and Brain Sciences 32:429-48. [AMaj]

Everett, D. L. (2005) Cultural constraints on grammar and cognition in Piraha: Another look at the design features of human language. Current Anthropology 46(4):621-46. [rJH] 
Falk, A. \& Heckman, J. J. (2009) Lab experiments are a major source of knowledge in the social sciences. Science 326(5952):535-38. [SG]

Falk, C. F., Heine, S. J., Yuki, M. \& Takemura, K. (2009) Why do Westerners selfenhance more than East Asians? European Journal of Personality 23(3):183203. $[\operatorname{arjH}]$

Farley, J. U., Lehmann, D. R. \& Ryan, M. J. (1981) Generalizing from "imperfect" replication. Journal of Business 54:597-610. [aJH]

Fehr, E., Fischbacher, U., von Rosenbladt, B., Schupp, J. \& Wagner, G. G. (2002) A nation-wide laboratory: Examining trust and trustworthiness by integrating behavioral experiments into representative surveys. Schmoller Fahrbuch 122:519-42. [aJH]

Fehr, E. \& Gächter, S. (1998) Reciprocity and economic: The economic implications of Homo reciprocans. European Economic Review 42(3-5):845-59. [aJH]

Fehr, E. \& Gächter, S. (2002) Altruistic punishment in humans. Nature 415:13740. [arJH]

Fehr, E., Gächter, S. \& Fischbacher, U. (2002) Strong reciprocity, human cooperation, and the enforcement of social norms. Human Nature 13(1):1-25. [SG]

Fehr, E. \& List, J. A. (2004) The hidden costs and returns of incentives: Trust and trustworthiness among CEOs. Journal of the European Economic Association 2(5):743-71. [aJH]

Fehr, E. \& Schmidt, K. M. (2006) The economics of fairness, reciprocity and altruism - Experimental evidence and new theories. In: Handbook of the economics of giving, altruism and reciprocity, ed. S.-C. Kolm \& J. M. Ythier. Elsevier. [SG]

Fernald, A. \& Marchman, V. A. (2006) Learning language in infancy. In: Handbook of psycholinguistics, 2nd edition, ed. M. Gernsbacher \& M. Traxler, pp. 1027 71. Academic Press. $[\mathrm{AF}]$

Fernald, A., Perfors, A. \& Marchman, V. (2006) Picking up speed in understanding: Speech processing efficiency and vocabulary growth across the second year. Developmental Psychology 42:98-116. [AF]

Fessler, D. M. T. (1999) Toward an understanding of the universality of second order emotions. In: Beyond nature or nurture: Biocultural approaches to the emotions, ed. A. Hinton, pp. 75-116. Cambridge University Press. [aJH]

Fessler, D. M. T. (2002) Reproductive immunosuppression and diet - An evolutionary perspective on pregnancy sickness and meat consumption. Current Anthropology 43(1):19-61. [rJH]

Fessler, D. M. T. (2004) Shame in two cultures: Implications for evolutionary approaches. Journal of Cognition and Culture 4(2):207-62. [DMTF, arJH]

Fessler, D. M. T. (2007) From appeasement to conformity: Evolutionary and cultural perspectives on shame, competition, and cooperation. In: The self-conscious emotions: Theory and research, ed. J. L. Tracy, R. W. Robins \& J. P. Tangney, pp. 174-93. Guilford Press. [DMTF]

Fessler, D. M. T. \& Machery, E. (forthcoming) Culture and cognition. In: Oxford handbook of philosophy and cognitive science, ed. E. Margolis, R. Samuels \& S. P. Stich. Oxford University Press. [EM]

Fessler, D. M. T., Nettle, D., Afshar, Y., Pinheiro, I. D., Bolyanatz, A., Mulder, M. B., Cravalho, M., Delgado, T., Gruzd, B., Correia, M. O., Khaltourina, D., Korotayev, A., Marrow, J., de Souza, L. S. \& Zbarauskaite, A. (2005) A crosscultural investigation of the role of foot size in physical attractiveness. Archives of Sexual Behavior 34(3):267-76. [aJH]

Fincher, C. L., Thornhill, R., Murray, D. \& Schaller, M. (2008) Pathogen prevalence predicts human cross-cultural variability in individualism/collectivism. Proceedings of the Royal Society B: Biological Science 275:1279-85. [SK]

Fiske, A. P. (1991) Structures of social life. Free Press. $\quad[\mathrm{rJH}]$

Fiske, A. P. (1993) Social errors in 4 cultures: Evidence about universal forms of social-relations. Journal of Cross-Cultural Psychology 24(4):463-94. [aJH]

Fiske, A. P. (1998) Learning a culture the way informants do: Observing, imitating, and participating. Unpublished manuscript, University of California-Los Angeles. Available at: http://www.bec.ucla.edu/papers/learning_culture.htm. [aJH, DFL]

Flaxman, S. M. \& Sherman, P. W. (2000) Morning sickness: A mechanism for protecting mother and embryo. Quarterly Review of Biology 75(2):113-48. $[\mathrm{rJH}]$

Flynn, J. R. (1987) Massive IQ gains in 14 nations: What IQ tests really measure. Psychological Bulletin 101:171-91. [aJH]

Flynn, J. R. (2007) What is intelligence? Beyond the Flynn effect. Cambridge University Press. [arjH]

Fodor, J. A. (1983) The modularity of mind: An essay on faculty psychology. MIT Press. [aJH]

Fouts, H. N. (2005) Families in Central Africa: A comparison of Bofi farmer and forager families. In: Families in global perspective, ed. J. L. Roopnarine, pp. 347-63. Pearson. [DFL]

Fouts, R. S., Hirsch, A. D. \& Fouts, D. H. (1982) Cultural transmission of a human language in a chimpanzee mother-infant relationship. In: Child nurturance: Studies of development in primates, ed. H. E. Fitzgerald, J. A. Mullins \& P. Gage, pp. 159-93. Plenum. [DAL]

Fowler, J. H. \& Kam, C. D. (2007) Beyond the self: Social identity, altruism, and political participation. Journal of Politics 69(3):813-27. [rJH]
Fox, A. T., Palmer, R. D. \& Davies, P. (2002) Do "Shufflebottoms" bottom shuffle? Archives of Disease in Childhood 87:552-54. [LBK]

Fox, J. R. (1973) Encounter with anthropology. Harcourt Brace Jovanovich. [rJH]

Fraga, M. F., Ballestar, E., Paz, M. F., Ropero, S., Setien, F., Ballestar, M. L. Heine-Sũner, D., Cigudosa, J. C., Urioste, M., Benitez, J., Boix-Chornet, M., Sanchez-Aguilera, A., Ling, C., Carlsson, E., Poulsen, P., Vaag, A., Stephan, Z., Spector, T. D., Wu, Y.-Z., Plass, C. \& Esteller, M. (2005) Epigenetic differences arise during the lifetime of monozygotic twins. Proceedings of the National Academy of Sciences USA 102(30):10604-09. [rJH]

Frankenburg, W. K. \& Dodds, J. B. (1967) The Denver developmental screening test. Journal of Pediatrics 71:181-91. [LBK]

Freeman, D., Orans, M. \& Cote, J. E. (2000) Forum on theory in anthropology: Sex and hoax in Samoa. Current Anthropology 41(4):609-22. [rJH]

Frohlich, N., Oppenheimer, J. \& Kurki, A. (2004) Modeling other-regarding preferences and an experimental test. Public Choice 119(1):91-117. [NB]

Fryberg, S. A. \& Markus, H. R. (2003) On being American Indian: Current and possible selves. Self and Identity 2:325-44. [aJH]

Gachter, S., Renner, E. \& Sefton, M. (2008) The long-run benefits of punishment. Science 322(5907):1510. [aJH]

Gaertner, L., Sedikides, C. \& Chang, K. (2008) On pancultural self-enhancement: Well-adjusted Taiwanese self-enhance on personally valued traits. Journal of Cross-Cultural Psychology 39:463-77. [LG]

Gagneux, P. \& Varki, A. (2001) Genetic differences between humans and great apes. Molecular Phylogetics and Evolution 18:2-13. [AMar]

Gangestad, S. W., Haselton, M. G. \& Buss, D. M. (2006) Evolutionary foundations of cultural variation: Evoked culture and mate preference. Psychological Inquiry 17:75-95. [aJH]

Garrigan, D. \& Hammer, M. (2006) Reconstructing human origins in the genomic era. Nature 7:669-80. [AMar]

Geertz, C. (1974) "From the native's point of view": On the nature of anthropological understanding. Bulletin of the American Academy of Arts and Sciences 28:26-45. [aJH]

Geertz, C. (1975) On the nature of anthropological understanding. American Scientist 63:4-53. [aJH]

Gelman, S. A. (2003) The essential child: Origins of essentialism in everyday thought. Oxford University Press. [aJH]

Gergen, K. J. (1973) Social psychology as history. Journal of Personality and Social Psychology 26:309-20. [aJH]

Gesell, A. (1928) Infancy and human growth. Macmillan. [LBK]

Gilbert, A. L., Regier, T., Kay, P. \& Ivry, R. B. (2006) Whorf hypothesis is supported in the right visual field but not the left. Proceedings of the National Academy of Sciences USA 103:489-94. [AMaj]

Gilbert, D. T. \& Malone, P. S. (1995) The correspondence bias. Psychological Bulletin 117:21-38. [aJH]

Gilby, I. C. (2006) Meat sharing among the Gombe chimpanzees: Harassment and reciprocal exchange. Animal Behaviour 71:953-63. [rJH]

Gilovich, T., Griffin, D. \& Kahneman, D., eds. (2002) Heuristics and biases: The psychology of intuitive judgment. Cambridge University Press. [arJH]

Gil-White, F. (2001) Are ethnic groups biological "species" to the human brain? Essentialism in our cognition of some social categories. Current Anthropology 42(4):515-54. [aJH]

Gislen, A., Dacke, M., Kroger, R. H. H., Abrahamsson, M., Nilsson, D.-E. \& Warrant, E. J. (2003) Superior underwater vision in a human population of sea gypsies. Current Biology 13(10):833-36. [rJH]

Gneezy, U. \& Rustichini, A. (2000) Pay enough or don't pay at all. Quarterly Journal of Economics 115(3):791-810. [SJC]

Godoy, R., Byron, E., Reyes-Garcia, V., Leonard, W. R., Patel, K., Apaza, L., Perez, E., Vadez, V. \& Wilkie, D. (2004) Patience in a foraging-horticultural society: A test of competing hypotheses. Journal of Anthropological Research 60(2):179_ 202. [aJH]

Goodall, J. (1986) The chimpanzees of Gombe: Patterns of behavior. The Belknap Press of Harvard University Press. [CB]

Gordon, P. (2004) Numerical cognition without words: Evidence from Amazonia. Science 306(5695):496-99. [arJH]

Göritz, A. S. (2010) Using lotteries, loyalty points, and other incentives to increase participant response and completion. In: Advanced methods for behavioral research on the Internet, ed. S. D. Gosling \& J. A. Johnson, pp. 219-33. American Psychological Association. [SDG]

Gosling, S. D. \& Johnson, J. A., eds. (2010) Advanced methods for behavioral research on the Internet. American Psychological Association. [SDG]

Gosling, S. D., Vazire, S., Srivastava, S. \& John, O. P. (2004) Should we trust Webbased studies? A comparative analysis of six preconceptions about Internet questionnaires. American Psychologist 59:93-104. [SDG]

Gottlieb, G. (2007) Probabilistic epigenesis. Developmental Science 10:1-11. [AF]

Graham, S. (1992) Most of the subjects were white and middle class: Trends in published research on African Americans in selected APA journals, 19701989. American Psychologist 47:629-39. [LBK] 
Greenberg, J., Solomon, S. \& Pyszczynski, T. (1997) Terror management theory of self-esteem and cultural worldviews: Empirical assessments and conceptual refinements. Advances in Experimental Social Psychology 29:61-139. [aJH]

Greenfield, P. M. (1966) On culture and conservation. In: Studies in cognitive growth, ed. J. S. Bruner, R. R. Oliver \& P. M. Greenfield, pp. 225-56. Wiley. [DFL]

Greenwald, A. G. \& Farnham, S. D. (2000) Using the Implicit Association Test to measure self-esteem and self-concept. Journal of Personality and Social Psychology 79:1022-38. [rJH]

Grossmann, I. (2010) Categorization differences between Americans and Russians. Unpublished manuscript. University of Michigan. [aJH]

Grossmann, I. \& Varnum, M. E. W. (2010) Social class, culture, and cognition. Manuscript under review. University of Michigan. [aJH]

Gurven, M. (2004) Does market exposure affect economic game behavior? The Ultimatum Game and the public goods game among the Tsimane of Bolivia. In: Foundations of human sociality: Economic experiments and ethnographic evidence from fifteen small-scale societies, ed. J. Henrich, R. Boyd, S. Bowles, C. Camerer, E. Fehr \& H. Gintis, pp. 194-231. Oxford University Press. [aJH]

Gurven, M. \& Winking, J. (2008) Collective action in action: Prosocial behavior in and out of the laboratory. American Anthropologist 110(2):179-90. [ [r]H, TSR]

Gutchess, A. H., Welsh, R. C., Boduroglu, A. \& Park, D. C. (2006) Cultural differences in neural function associated with object processing. Cognitive, Affective and Behavioral Neuroscience 6:102-109. [JYC, aJH]

Guth, W., Schmidt, C. \& Sutter, M. (2003) Fairness in the mail and opportunism in the Internet: A newspaper experiment on ultimatum bargaining. Economic Review 4(2):456-75. [aJH]

Hackman, D. A. \& Farah, M. J. (2009) Socioeconomic status and the developing brain. Trends in Cognitive Sciences 13(2):65-73. [JYC]

Hagen, E. H. \& Hammerstein, P. (2006) Game theory and human evolution: A critique of some recent interpretations of experimental games. Theoretical Population Biology 69(3):339-48. [NB]

Haidt, J. \& Graham, J. (2007) When morality opposes justice: Conservatives have moral intuitions that liberals may not recognize. Social Justice Research 20:98116. [aJH]

Haidt, J., Koller, S. H. \& Dias, M. G. (1993) Affect, culture, and morality, or is it wrong to eat your dog. Journal of Personality and Social Psychology 65(4):61328. [aJH]

Hamamura, T., Heine, S. J. \& Paulhus, D. L. (2008) Cultural differences in response styles: The role of dialectical thinking. Personality and Individual Differences 44:932-42. [aJH]

Hamamura, T., Heine, S. J. \& Takemoto, T. (2007) Why the better-than-average effect is a worse-than-average measure of self-enhancement. An investigation of conflicting findings from studies of East Asian self-evaluations. Motivation and Emotion 31:247-59. [rJH]

Haritatos, J. \& Benet-Martínez, V. (2002) Bicultural identities: The interface of cultural, personality, and socio-cognitive processes. Journal of Research in Personality 36:598-606. [SSK]

Harrington, L. \& Liu, J. H. (2002) Self-enhancement and attitudes toward high achievers: A bicultural view of the independent and interdependent self. Journal of Cross-Cultural Psychology 33:37-55. [aJH]

Harrison, G. W., Lau, M. I. \& Williams, M. B. (2002) Estimating individual discount rates in Denmark: A field experiment. American Economic Review 92(5):1606-17. [aJH]

Haslanger, S. (2008) Changing the ideology and culture of philosophy: Not by reason (alone). Hypatia 23(2):210-23. [SS]

Haun, D. B. M., Call, J., Janzen, G. \& Levinson, S. C. (2006) Evolutionary psychology of spatial representations in the hominidae. Current Biology 16(17):1736-40. [aJH]

Haun, D. B. M. \& Rapold, C. J. (2009) Variation in memory for body movements across cultures. Current Biology 19:R1068-69. [AMaj]

Haun, D. B. M., Rapold, C. J., Call, J., Janzen, G. \& Levinson, S. C. (2006) Cognitive cladistics and cultural override in hominid spatial cognition. Proceedings of the National Academy of Sciences USA 103(46):17568-73. [arJH]

Hauser, M. D. \& Spelke, E. S. (2004) Evolutionary and developmental foundations of human knowledge: A case study of mathematics. In: The cognitive neurosciences, ed. M. Gazzaniga, pp. 853-64. MIT Press. [rJH]

Hawthorne, J. (2004) Knowledge and lotteries. Oxford University Press. [SS]

Hedden, T., Ketay, S., Aron, A., Markus, H. R. \& Gabrieli, J. D. E. (2008) Cultural influences on neural substrates of attentional control. Psychological Science 19(1):12-17. [JYC, aJH]

Heglund, N. C., Willems, P. A., Penta, M. \& Cavagna, G. A. (1995) Energy-saving

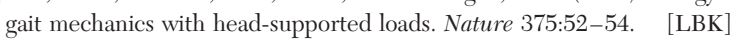

Heine, S. J. (2005) Where is the evidence for pancultural self-enhancement? A reply to Sedikides, Gaertner, \& Toguchi. Journal of Personality and Social Psychology 89:531-38. [r]H]

Heine, S. J. (2008) Cultural psychology. W. W. Norton. [aJH]
Heine, S. J. \& Hamamura, T. (2007) In search of East Asian self-enhancement. Personality and Social Psychology Review 11:1-24. [arJH]

Heine, S. J., Kitayama, S. \& Hamamura, T. (2007a) The inclusion of additional studies yields different conclusions: A reply to Sedikides, Gaertner \& Vevea, 2005, JPSP. Asian Journal of Social Psychology 10:49-58. [rJH]

Heine, S. J., Kitayama, S. \& Hamamura, T. (2007b) Which studies test the question of pancultural self-enhancement? A reply to Sedikides, Gaertner \& Vevea, 2007. Asian Journal of Social Psychology 10:198-200. [rJH]

Heine, S. J., Kitayama, S., Lehman, D. R., Takata, T., Ide, E., Leung, C. \& Matsumoto, H. (2001) Divergent consequences of success and failure in Japan and North America: An investigation of self-improving motivations and malleable selves. Journal of Personality and Social Psychology 81:599-615. [rJH]

Heine, S. J. \& Lehman, D. R. (1999) Culture, self-discrepancies, and self-satisfaction. Personality and Social Psychology Bulletin 25:915-25. [rJH]

Heine, S. J., Lehman, D. R., Markus, H. R. \& Kitayama, S. (1999) Is there a universal need for positive self-regard? Psychological Review 106:766-94. [LG]

Heine, S. J., Lehman, D. R., Peng, K. \& Greenholtz, J. (2002) What's wrong with cross-cultural comparisons of subjective Likert scales? The reference-group problem. Journal of Personality and Social Psychology 82:903-18. [aJH]

Heine, S. J. \& Raineri, A. (2009) Self-improving motivations and culture: The case of Chileans. Journal of Cross-Cultural Psychology 40:158-63. [aJH]

Heine, S. J., Takata, T. \& Lehman, D. R. (2000) Beyond self-presentation: Evidence for self-criticism among Japanese. Personality and Social Psychology Bulletin 26:71-78. [rJH]

Heintz, C. (2005) The ecological rationality of strategic cognition. Behavioral and Brain Sciences 28(6):825-26. [NB]

Hennig-Schmidt, H., Li, Z.-Y. \& Yang, C. (2008) Why people reject advantageous offers: Non-monotone strategies in ultimatum bargaining: First results from a video experiment in the People's Republic of China. Journal of Economic Behavior and Organization 65:373-84. [aJH]

Henrich, J. (2008) A cultural species. In: Explaining culture scientifically, ed. M. Brown, pp. 184-210. University of Washington Press. [arJH]

Henrich, J., Boyd, R., Bowles, S., Camerer, C. F., Fehr, E., Gintis, H., McElreath, R., Alvard, M., Barr, A., Ensminger, J., Henrich, N. S., Hill, K., Gil-White, F., Gurven, M., Marlowe, F. W., Patton, J. Q. \& Tracer, D. (2005a) "Economic man" in cross-cultural perspective: Behavioral experiments in 15 small-scale societies. Behavioral and Brain Sciences 28(6):795-815; discussion 815-55. [NB, SG, arJH, SK, EM, RAS]

Henrich, J., Boyd, R., Bowles, S., Camerer, C. F., Fehr, E., Gintis, H., McElreath, R., Alvard, M., Barr, A., Ensminger, J., Henrich, N. S., Hill, K., Gil-White, F., Gurven, M., Marlowe, F. W., Patton, J. Q. \& Tracer, D. (2005b) Models of decision-making and the coevolution of social preferences. [Authors' Response]. Behavioral and Brain Sciences 28(6):838-55. [rJH]

Henrich, J., Boyd, R., Bowles, S., Gintis, H., Camerer, C., Fehr, E. \& McElreath, R. (2001) In search of Homo economicus: Experiments in 15 small-scale societies. American Economic Review 91:73-78. [rJH]

Henrich, J., Boyd, R., Bowles, S., Gintis, H., Fehr, E. \& Camerer, C., eds. (2004) Foundations of human sociality: Economic experiments and ethnographic evidence from fifteen small-scale societies. Oxford University Press. $[\mathrm{rJH}$, $\mathrm{EM}]$

Henrich, J. \& Ensminger, J., eds. (n. d.) Experimenting with social norms: Fairness and punishment in cross-cultural perspective. Unpublished manuscript, University of Bristish Columbia. [r]H]

Henrich, J., Ensminger, J., McElreath, R., Barr, A., Barrett, C., Bolyanatz, A. Cardenas, J. C., Gurven, M., Gwako, E., Henrich, N., Lesorogol, C., Marlowe, F., Tracer, D. P. \& Ziker, J. (2010) Market, religion, community size and the evolution of fairness and punishment. Science 327(5972):1480-84. [arJH]

Henrich, J. \& Gil-White, F. (2001) The evolution of prestige: Freely conferred deference as a mechanism for enhancing the benefits of cultural transmission. Evolution and Human Behavior 22(3):165-96. [rJH]

Henrich, J. \& Henrich, N. (under review) Fairness without punishment: Behavioral experiments in the Yasawa Island, Fiji. In: Experimenting with social norms: Fairness and punishment in cross-cultural perspective, ed. J. Henrich \& J. Ensminger. Russell Sage Foundation Press. [aJH]

Henrich, J. \& McElreath, R. (2002) Are peasants risk-averse decision makers? Current Anthropology 43(1):172-81. [aJH]

Henrich, J., McElreath, R., Ensminger, J., Barr, A., Barrett, C., Bolyanatz, A. Cardenas, J. C., Gurven, M., Gwako, E., Henrich, N., Lesorogol, C., Marlowe, F., Tracer, D. \& Ziker, J. (2006) Costly punishment across human societies. Science 312(5868):1767-70. [CB, arJH $]$

Henrich, J. \& Smith, N. (2004) Comparative experimental evidence from Machiguenga, Mapuche, and American populations. In: Foundations of human sociality: Economic experiments and ethnographic evidence from fifteen smallscale societies, ed. J. Henrich, R. Boyd, S. Bowles, H. Gintis, E. Fehr \& C. Camerer, pp. 123-67. Oxford University Press. [arJH]

Henrich, N. \& Henrich, J. (2007) Why humans cooperate: A cultural and evolutionary explanation. Oxford University Press. [arjH] 
Henry, P. J. (2009) College sophomores in the laboratory redux: Influences of a narrow database on social psychology's view of the nature of prejudice. Psychological Inquiry 19:49-71. [aJH]

Herdt, G. H., eds. (1984/1993) Ritualized homosexuality in Melanesia. University of California Press. [aJH]

Herrmann, B., Thoni, C. \& Gächter, S. (2008) Antisocial punishment across societies. Science 319(5868):1362-67. [SG, arJH]

Herrmann, E., Call, J., Lloreda, M., Hare, B. \& Tomasello, M. (2007) Humans have evolved specialized skills of social cognition: The cultural intelligence hypothesis. Science 317:1360-66. [CB]

Hespos, S. J. \& Spelke, E. S. (2002) Conceptual precursors to language. Nature 430:453-56. [AMaj]

Hill, K. \& Gurven, M. (2004) Economic experiments to examine fairness and cooperation among the Ache Indians of Paraguay. In: Foundations of human sociality: Economic experiments and ethnographic evidence from fifteen smallscale societies, ed. J. Henrich, R. Boyd, S. Bowles, C. Camerer, H. Gintis \& E Fehr, pp. 382-412. Oxford University Press. [rJH]

Hoff, E. (2003) The specificity of environmental influence: Socioeconomic status affects early vocabulary development via maternal speech. Child Development 74:1368-78. [AF]

Hoffman, D. D. \& Richards, W. A. (1984) Parts of recognition. Cognition 18:6596. [AMaj]

Hoffman, E., McCabe, K., Shachat, K. \& Smith, V. (1994) Preferences, property rights, and anonymity in bargaining experiments. Games and Economic Behavior 7:346-80. [EM]

Hoffman, E., McCabe, K. \& Smith, V. (1998) Behavioral foundations of reciprocity: Experimental economics and evolutionary psychology. Economic Inquiry 36:335-52. [aJH]

Hofmann, W., Gawronski, B., Gschwendner, T., Le, H. \& Schmitt, M. (2005) A meta-analysis on the correlation between the implicit association test and explicit self-report measures. Personality and Social Psychology Bulletin 31:1369-85. [rJH]

Hofstede, G. (1980) Culture's consequences: International differences in workrelated values. Sage. [aJH]

Hofstede, G. (2001) Culture's consequences: Comparing values, behaviors, institutions, and organizations across nations. Sage. [aJH]

Hong, L. \& Page, S. E. (2004) Groups of diverse problem solvers can outperform groups of high-ability problem solvers. Proceedings of the National Academy of Sciences USA 101(46):16385-89. [MM]

Hong, Y.-Y., Morris, M. W., Chiu, C.-Y. \& Benet-Martínez, V. (2000) Multicultural minds: A dynamic constructivist approach to culture and cognition. American Psychologist 55:709-20. [SSK]

Hopkins, B. \& Westra, T. (1988) Maternal handling and motor development: An intracultural study. Genetic, Social and General Psychology Monographs 114:379-408. [LBK]

Hopkins, W. D., Taglialatela, J. \& Leavens, D. A. (2007) Chimpanzees differentially produce vocalizations to capture the attention of a human. Animal Behaviour 73:281-86. [DAL]

Horner, V. \& Whiten, A. (2005) Causal knowledge and imitation/emulation switching in chimpanzees (Pan troglodytes) and children (Homo sapiens). Animal Cognition 8(3):164-81. [rJH]

Hostetter, A., Cantero, M. \& Hopkins, W. D. (2001) Differential use of vocal and gestural communication in chimpanzees in response to the attentional status of a human audience. Journal of Comparative Psychology 115:33743. [DAL]

Hsu, M., Krajbich, I., Zhao, C. \& Camerer, C. F. (2009) Neural response to reward anticipation under risk is nonlinear in probabilities. Journal of Neuroscience 29(7):2231-37. [aJH]

Humphrey, N., Skoyles, J. \& Keynes, R. (2005) Human hand-walkers: Five siblings who never stood up. Discussion Paper. Centre for Philosophy of Natural and Social Science, London, United Kingdom. [LBK

Humphrey, S. J. \& Verschoor, A. (2004a) Decision-making under risk among small farmers in East Uganda. Journal of African Economies 13(1):44-101. [aJH]

Humphrey, S. J. \& Verschoor, A. (2004b) The probability weighting function: Experimental evidence from Uganda, India and Ethiopia. Economics Letters 84(3):419-25. [aJH]

Hurtado, N., Marchman, V. A. \& Fernald, A. (2007) Spoken word recognition by Latino children learning Spanish as their first language. Journal of Child Language 33:27-249. [AF]

Hurtado, N., Marchman, V. A. \& Fernald, A. (2008) Does input influence uptake? Links between maternal talk, processing speed and vocabulary size in Spanishlearning children. Developmental Science 11:F31-39. [AF]

Hyde, J. S. (1981) How large are cognitive gender differences? American Psychologist 36:892-901. [aJH]

Inglehart, R., Basanez, M. \& Moreno, A. (1998) Human values and beliefs: A crosscultural sourcebook. University of Michigan Press. [aJH]

Inglehart, R. \& Welzel, C. (2005) Modernization, cultural change and democracy. Cambridge University Press. [MM]
Inoue-Nakamura, N. \& Matsuzawa, T. (1997) Development of stone tool use by wild chimpanzees (Pan troglodytes). Journal of Comparative Psychology 111:159-73. [DAL]

Itakura, S. \& Tanaka, M. (1998) Use of experimenter-given cues during objectchoice tasks by chimpanzees (Pan troglodytes), an orangutan (Pongo pygmaeus), and human infants (Homo sapiens). Journal of Comparative Psychology 112:119-26. [DAL]

Iyengar, S. S. \& DeVoe, S. E. (2003) Rethinking the value of choice: Considering cultural mediators of intrinsic motivation. In: Nebraska symposium on motivation: vol. 49. Cross-cultural differences in perspectives on the self, ed. V. Murphy-Berman \& J. J. Berman. pp. 129-74. University of Nebraska Press. [aJH]

Iyengar, S. S. \& Lepper, M. R. (1999) Rethinking the value of choice: A cultural perspective on intrinsic motivation. Journal of Personality and Social Psychology 76:349-66. [aJH]

Jablonka, E. \& Lamb, M. (2005) Evolution in four dimensions. MIT Press. [KP]

Jablonka, E. \& Raz, G. (2009) Transgenerational epigenetic inheritance: Prevalence, mechanisms, and implications for the study of heredity and evolution. The Quarterly Review of Biology 84(2):131-76. [rJH]

Jensen, K., Call, J. \& Tomasello, M. (2007) Chimpanzees are rational maximizers in an Ultimatum Game. Science 318(5847):107-109. [aJH]

Jensen, K., Hare, B., Call, J. \& Tomasello, M. (2006) What's in it for me? Self-regard precludes altruism and spite in chimpanzees. Proceedings of the Royal Society of London, B - Biological Sciences 273(1589):1013-21. [rJH]

Jensen, L. A. (1997) Culture wars: American moral divisions across the adult lifespan. Journal of Adult Development 4:107-21. [aJH]

Ji, L., Nisbett, R. E. \& Zhang, Z. (2004) Is it culture or is it language? Examination of language effects in cross-cultural research on categorization. Journal of Personality and Social Psychology 87:57-65. [aJH]

Ji, L., Peng, K. \& Nisbett, R. E. (2000) Culture, control and perception of relationships in the environment. Journal of Personality and Social Psychology 78:943-55. [aJH]

Johnson, J. A. (2010) Web-based self-report personality scales. In: Advanced methods for behavioral research on the Internet, ed. S. D. Gosling \& J. A. Johnson, pp. 149-66. American Psychological Association. [SDG]

Johnson, J. A. \& Gosling, S. D. (2010) How to use this book. In: Advanced methods for behavioral research on the Internet, ed. S. D. Gosling \& J. A. Johnson, pp. 1-8. American Psychological Association. [SDG]

Johnson-Laird, P. N. (1983) Mental models. Harvard University Press. [SJC]

Johnson-Laird, P. N. (2006) How we reason. Oxford University Press. [SSK

Johnson-Laird, P. N., Legrenzi, P., Girotto, V. \& Legrenzi, M. (2000) Illusions in reasoning about consistency. Science 288:531-32. [SSK]

Jones, E. E. \& Harris, V. A. (1967) The attribution of attitudes. Journal of Experimental Social Psychology 3:1-24. [aJH]

Kaessmann, H. \& Paabo, S. (2002) The genetical history of humans and the great apes. Journal of Internal Medicine 252:1-18. [AMar]

Kaessmann, H., Wiebe, V. \& Paabo, S. (1999) Extensive nuclear DNA sequence diversity among chimpanzees. Science 286:1159-61. [AMar]

Kahan, D. M. (in press) Culture, cognition, and consent: Who perceives what, and why, in "acquaintance rape" cases. University of Pennsylvania Law Review. [SJC $]$

Kahan, D. M., Braman, D., Gastil, J., Slovic, P. \& Mertz, C. K. (2007) Culture and identity-protective cognition: Explaining the white-male effect in risk perception. Journal of Empirical Legal Studies 4(3):465-505. [SJC]

Kahan, D. M., Hoffman, D. A. \& Braman, D. (2009) Whose eyes are you going to believe? Scott v. Harris and the perils of cognitive illiberalism. Harvard Law Review 122:837. [SJC]

Kahneman, D., Slovic, P. \& Tversky, A. (1982) Judgment under uncertainty: Heuristics and biases. Cambridge University Press. [aJH]

Kahneman, D. \& Tversky, A. (2000) Choices, values, and frames. Cambridge University Press. [aJH]

Kam, C. D., Cranmer, S. J. \& Fowler, J. H. (n.d.) When it's not all about me: Altruism, participation, and political context. Unpublished manuscript. $[\mathrm{rJH}]$

Kanagawa, C., Cross, S. E. \& Markus, H. R. (2001) "Who am I?": The cultural psychology of the conceptual self. Personality and Social Psychology Bulletin 27:90-103. [aJH]

Kanovsky, M. (2007) Essentialism and folksociology: Ethnicity again. Journal of Cognition and Culture 7:241-81. [aJH]

Karasik, L. B., Bornstein, M. H., Suwalsky, J. T. D., Zuckerman, A., Adolph, K. E. \& Tamis-LeMonda, C. S. (2010) Places, parenting, postures, and pieces: A cross-cultural comparison of mothers and their 5-month-olds' motor development and object exploration. Poster presented at the meeting of the Society for Cross-Cultural Research, Albuquerque, NM, February 17-20, 2010. [LBK]

Karlan, D. S. (2005) Using experimental economics to measure social capital and predict financial decisions. American Economic Review 95(5):1688-99. $[\mathrm{rJH}]$ 
Kay, P. (2005) Color categories are not arbitrary. Cross-Cultural Research 39(1):39-55. [aJH]

Kay, P. \& Maffi, L. (2008) Number of basic color categories. In: The world atlas of language structures online, ed. M. Haspelmath, M. S. Dryer, D. Gil \& B. Comrie, Ch. 133. Max Planck Digital Library. [Available at: http://wals.info/ feature/133. Accessed December 17, 2009.] [AMaj]

Kay, P. \& Regier, T. (2006) Language, thought and color: Recent developments. Trends in Cognitive Sciences 10(2):51-54. [aJH]

Keel, P. K. \& Klump, K. L. (2003) Are eating disorders culture-bound syndromes? Implications for conceptualizing their etiology. Psychological Bulletin 129:747-69. [rJH]

Kelley, R. C. (1980) Etoro social structure: A study in structural contradiction. University of Michigan Press. [aJH]

Kellogg, W. N. \& Kellogg, L. A. (1933) The ape and the child: A study of early environmental influence upon early behavior. McGraw-Hill. [DAL]

Kelly, M. (1971) Some aspects of conservation of quantity and length in Papua New Guinea. PNG Journal of Education 7(1):55-60. [DFL]

Kennedy, S., Scheirer, J. \& Rogers, A. (1984) International education in psychology: The price of success - our monocultural science. American Psychologist 39:996-97. [LBK]

Kenrick, D. T. \& Keefe, R. C. (1992a) Age preferences in mates reflect sexdifferences in reproductive strategies. Behavioral and Brain Sciences 15(1):75-133. [aJH]

Kenrick, D. T. \& Keefe, R. C. (1992b) Sex-differences in age preference Universal reality or ephemeral construction. [Authors' response.] Behavioral and Brain Sciences 15(1):119-33. [aJH]

Kent, M. M. \& Haub, C. (2005) Global demographic divide. Population Bulletin 60(4):1-24. [PRoc]

Kessler, R. C., McGonagle, K. A., Zhao, S., Nelson, C. B., Hughes, N., Eshleman, S., Wittchen, H.-U. \& Kendler, K. S. (1994) Lifetime and 12-month prevalence of DSM-III-R psychiatric disorders in the United States: Results from the National Comorbidity Survey. Archives of General Psychiatry 51:8-19. $[\mathrm{rJH}]$

Kim, H. \& Markus, H. R. (1999) Deviance or uniqueness, harmony or conformity? A cultural analysis. Journal of Personality and Social Psychology 77:785800. [aJH, SK]

Kim, H. S., Sherman, D. K., Taylor, S. E., Sasaki, J. Y., Chu, T. Q., Ryu, C. \& Xu, J. (in press) Culture, serotonin receptor polymorphism (5-HTR1A), and locus of attention. Social Cognitive and Affective Neuroscience. [aJH]

Kirby, K. N., Godoy, R., Reyes-Garcia, V., Byron, E., Apaza, L., Leonard, W., Perez, E., Vadez, V. \& Wilkie, D. (2002) Correlates of delay-discount rates: Evidence from Tsimane' Amerindians of the Bolivian rain forest. Journal of Economic Psychology 23(3):291-316. [aJH]

Kitayama, S., Duffy, S., Kawamura, T. \& Larsen, J. T. (2003) Perceiving an object and its context in different cultures: A cultural look at new look. Psychological Science 14:201-206. [aJH]

Kitayama, S., Ishii, K., Imada, T., Takemura, K. \& Ramaswamy, J. (2006) Voluntary settlement and the spirit of independence: Evidence from Japan's "Northern Frontier." Journal of Personality and Social Psychology 91:369-84. [SK]

Kitayama, S. \& Markus, H. R., eds. (1994) Emotion and culture: Empirical studies of mutual influence. American Psychological Association. [DMTF]

Kitayama, S., Park, H., Sevincer, A. T., Karasawa, M. \& Uskul, A. K. (2009) A cultural task analysis of implicit independence: Comparing North America, Western Europe, and East Asia. Journal of Personality and Social Psychology $97: 236-55 . \quad[\mathrm{aJH}]$

Klar, Y. \& Giladi, E. E. (1997) No one in my group can be below the group's average: A robust positivity bias in favor of anonymous peers. Journal of Personality and Social Psychology 73:885-901. [rJH]

Kleinman, A. (1988) Rethinking psychiatry: From cultural category to personal experience. Free Press. [arJH]

Knowles, E. D., Morris, M. W., Chiu, C. \& Hong, Y. (2001) Culture and the process of person perception: Evidence for automaticity among East Asians in correcting for situational influences on behavior. Personality and Social Psychology Bulletin 27:1344-56. [aJH]

Kobayashi, C. \& Brown, J. D. (2003) Self-esteem and self-enhancement in Japan and America. Journal of Cross-Cultural Psychology 34:567-80. [LG]

Kohlberg, L. (1971) From is to ought: How to commit the naturalistic fallacy and get away with it in the study of moral development. In: Cognitive development and epistemology, ed. L. Mischel, pp. 151-284. Academic Press. [aJH]

Kohlberg, L. (1976) Moral stages and moralization. In: Moral development and behavior: Theory, research and social issues, ed. T. Lickona, pp. 31-53. Holt, Rinehart and Winston. [aJH]

Kohlberg, L. (1981) The philosophy of moral development. Harper \& Row. [aJH]

Konečni, V. J. \& Ebbesen, E. B. (1982a) An analysis of the sentencing system. In The criminal justice system: A social-psychological analysis, ed. V. J. Konečni \& E. B. Ebbesen, pp. 293-332. Freeman. [VJK]

Konečni, V. J. \& Ebbesen, E. B., eds. (1982b) The criminal justice system: A socialpsychological analysis, pp. 413-23. Freeman.
Konečni, V. J. \& Ebbesen, E. B. (1992) Methodological issues in research on legal decision-making, with special reference to experimental simulations. In: Psychology and law, ed. F. Lösel, D. Bender \& T. Bliesener. Walter de Gruyter. $[\mathrm{VJK}]$

Konečni, V. J., Ebbesen, E. B. \& Konečni, D. K. (1976) Decision processes and risk taking in traffic: Driver response to the onset of yellow light. Journal of Applied Psychology 61:359-67. [VJK]

Krantz, J. H. \& Williams, J. H. (2010) Using graphics, photographs, and dynamic media. In: Advanced methods for behavioral research on the internet, ed. S. D. Gosling \& J. A. Johnson, pp. 45-61. American Psychological Association. [SDG]

Kraul, C. (2008) Environmental pollution and encroaching narco have taken their toll on Narino's state's Afro-Columbians. The community's unique culture, and altruism, is in peril. Los Angeles Times, April 14, 2008, p. 1. [arJH]

Krause, M. A. \& Fouts, R. S. (1997) Chimpanzee (Pan troglodytes) pointing: Hand shapes, accuracy, and the role of eye gaze. Journal of Comparative Psychology 111:330-36. [DAL]

Krizan, X. \& Suls, J. (2008) Losing sight of oneself in the above-average effect: When egocentrism, focalism, and group diffuseness collide. Journal of Experimental Social Psychology 44:929-42. [rJH]

Krosnick, J. A. \& Alwin, D. F. (1989) Aging and susceptibility to attitude change. Journal of Personality and Social Psychology 57:416-25. [aJH]

Kruger, J. (1999) Lake Wobegon be gone! The "below-average effect" and the egocentric nature of comparative ability judgments. Journal of Personality and Social Psychology 77:221-32. [rJH]

Kuhn, M. H. \& McPartland, T. (1954) An empirical investigation of self-attitudes. American Sociological Review 19:68-76. [aJH]

Kuhnen, U., Hannover, B., Roeder, U., Shah, A. A., Schubert, B., Upmeyer, A. \& Zakaria, S. (2001) Cross-cultural variations in identifying embedded figures Comparisons from the United States, Germany, Russia, and Malaysia. Journal of Cross-Cultural Psychology 32(3):365-71. [aJH]

Kurman, J. (2003) Why is self-enhancement low in certain collectivist cultures? An investigation of two competing explanations. Journal of Cross-Cultural Psychology 34:496-510. [LG]

Kusserow, A. S. (1999) De-homogenizing American individualism: Socializing hard and soft individualism in Manhattan and Queens. Ethos 27:210-34. [aJH]

Kyburg, H. (1961) Probability and the logic of rational belief. Wesleyan University Press. [SS]

Lakhani, K. R., Jeppesen, L. B., Lohse, P. A. \& Panetta, J. A. (2006) The value of openness in scientific problem solving. Harvard Business School Working Paper. Available at: http://www.hbs.edu/research/pdf/07-050.pdf. [MM]

Laland, K. N., Odling-Smee, J. \& Feldman, M. W. (2000) Niche construction, biological evolution, and cultural change. Behavioral and Brain Sciences 23:131-75. [WMB]

Laland, K. N., Odling-Smee, J. \& Myles, S. (2010) How culture shaped the human genome: Bringing genetics and the human sciences together. Nature Reviews Genetics 11(2):137-48. [rJH]

Lamont, M. (2000) The dignity of working men. Russell Sage Foundation. [aJH]

Lancy, D. F. (1983) Cross-cultural studies in cognition and mathematics. Academic Press. [DFL]

Lancy, D. F. (1996) Playing on mother ground: Cultural routines for children's development. Guilford. [aJH]

Lancy, D. F. (2007) Accounting for the presence/absence of mother-child play. American Anthropologist 109(2):273-84. [DFL]

Lancy, D. F. (2008) The anthropology of childhood: Cherubs, chattel and changelings. Cambridge University Press. [arJH]

Lancy, D. F. \& Grove, M. A. (2010) Learning guided by others. In: The anthropology of learning in childhood, ed. D. F. Lancy, S. Gaskins \& J. Bock, pp. 145-79. Alta-Mira. [DFL]

Lancy, D. F. \& Madsen, M. C. (1981) Cultural patterns and the social behavior of children: Two studies from Papua New Guinea. Ethos 9:201-16. [DFL]

Lancy, D. F. \& Strathern, A. J. (1981) Making-twos: Pairing as an alternative to the taxonomic mode of representation. American Anthropologist 81:773-95. [DFL]

Leavens, D. A. (2002) On the public nature of communication. Behavioral and Brain Sciences 25:630-31. [DAL]

Leavens, D. A. (2004) Manual deixis in apes and humans. Interaction Studies 5:387-408. [Reprinted in: C. Abry, A. Vilain \& J-L. Schwartz, eds. (2009) Vocalize to localize, pp. 67-86. John Benjamins.] [DAL]

Leavens, D. A. (2006) It takes time and experience to learn how to interpret gaze in mentalistic terms. Infant and Child Development 9:187-90. [DAL]

Leavens, D. A. \& Hopkins, W. D. (1998) Intentional communication by chimpanzees: A cross-sectional study of the use of referential gestures. Developmental Psychology 34:813-22. [DAL]

Leavens, D. A., Hopkins, W. D. \& Bard, K. A. (1996) Indexical and referential pointing in chimpanzees (Pan troglodytes). Journal of Comparative Psychology 110:346-53. [DAL] 
Leavens, D. A., Hopkins, W. D. \& Bard, K. A. (2005a) Understanding the point of chimpanzee pointing: Epigenesis and ecological validity. Current Directions in Psychological Science 14:185-89. [DAL]

Leavens, D. A., Hopkins, W. D. \& Bard, K. A. (2008) The heterochronic origins of explicit reference. In: The shared mind: Perspectives on intersubjectivity, ed. J. Zlatev, T. Racine, C. Sinha \& E. Itkonen, pp. 187-214. John Benjamins. [DAL]

Leavens, D. A., Hopkins, W. D. \& Thomas, R. K. (2004a) Referential communication by chimpanzees (Pan troglodytes). Journal of Comparative Psychology 118:48-57. [DAL]

Leavens, D. A., Hostetter, A. B., Wesley, M. J. \& Hopkins, W. D. (2004b) Tactical use of unimodal and bimodal communication by chimpanzees, Pan troglodytes. Animal Behaviour 67:467-76. [DAL]

Leavens, D. A. \& Racine, T. P. (2009) Joint attention in apes and humans: Are humans unique? Journal of Consciousness Studies 16:240-67. [DAL]

Leavens, D. A., Racine, T. P. \& Hopkins, W. D. (2009) The ontogeny and phylogeny of non-verbal deixis. In: The prehistory of language, ed. R. Botha \& C. Knight, pp. 142-65. Oxford University Press. [DAL]

Leavens, D. A., Russell, J. L. \& Hopkins, W. D. (2005b) Intentionality as measured in the persistence and elaboration of communication by chimpanzees (Pan troglodytes). Child Development 76:291-306. [DAL]

Leavens, D. A., Russell, J. L. \& Hopkins, W. D. (2010) Multimodal communication by captive chimpanzees (Pan troglodytes). Animal Cognition 13:33-40. [DAL]

Lee, A. Y., Aaker, J. L. \& Gardner, W. L. (2000) The pleasures and pains of distinct self-construals: The role of interdependence in regulatory focus. Journal of Personality and Social Psychology 78:1122-34. [aJH]

Lesorogol, C. (2007) Bringing norms in. Current Anthropology (48)920-26. [NB]

Lesorogol, C. \& Ensminger, J. (under review) Double-blind dictator games in Africa and the U.S.: Differential experimenter effects. In: Experimenting with social norms, ed. J. Henrich \& J. Ensminger. [aJH]

LeVine, R. A. (2004) Challenging expert knowledge: Findings from an African study of infant care and development. In: Childhood and adolescence: Cross-cultural perspectives and applications, ed. U. P. Gielen \& J. L. Roopnarine, pp. 149 65. Praeger. [DFL]

LeVine, R. A. \& Norman, K. (2001) The infant's acquisition of culture: Early attachment reexamined in anthropological perspective. In: The psychology of cultural experience, ed. C. C. Moore \& H. F. Mathews, pp. 83-104. Cambridge University Press. [TSR]

Levine, S. C., Vasilyeva, M., Lourenco, S. F., Newcombe, N. S. \& Huttenlocher, J. (2005) Socioeconomic status modifies the sex difference in spatial skill. Psychological Science 16(11):841-45. [AF, aJH]

Levinson, S. C. (2003) Space in language and cognition. Cambridge University Press. [aJH]

Levinson, S. C., Kita, S., Haun, D. \& Rasch, B. H. (2002) Returning the tables: Language affects spatial reasoning. Cognition 84:155-88. [aJH]

Levitt, S. D. \& List, J. A. (2007) What do laboratory experiments measuring social preferences reveal about the real world? Journal of Economic Perspectives $2 \mathrm{l}(2): 153-74 . \quad[\mathrm{rJH}]$

Levy, R. I. (1973) Tahitians: Mind and experience in the Society Islands. University of Chicago Press. [DMTF]

Lewis, C. C. (1995) Educating hearts and minds. Cambridge University Press. $[\mathrm{aJH}]$

Lewontin, R. C. (1998) The evolution of cognition: Questions we will never answer. In: An invitation to cognitive science: Methods, models, and conceptual issues, vol. 4, ed. D. Scarborough \& S. Sternberg, pp. 107-32. MIT Press. [KP]

Li, P. \& Gleitman, L. (2002) Turning the tables: Language and spatial reasoning. Cognition 83:265-94. [aJH, AMaj]

Liberman, V., Samuels, S. M. \& Ross, L. (2004) The name of the game: Predictive power of reputations versus situational labels in determining Prisoner's Dilemma Game moves. Personality and Social Psychology Bulletin 30(9):1175-85. [SJC]

Lieberman, D. E., Venkadesan, M., Werbel, W. A., Daoud, A. I., D’Andrea, S., Davis, I. S., Mang'Eni, R. O. \& Pitsiladis, Y. (2010) Foot strike patterns and collision forces in habitually barefoot versus shod runners. Nature 463(7280):531-35. [rJH]

Lieberman, M. D., Jarcho, J. M. \& Obayashi, J. (2005) Attributional inference across cultures: Similar automatic attributions and different controlled corrections. Personality and Social Psychology Bulletin 31:889-901. [aJH]

Lipset, S. M. (1996) American exceptionalism: A double-edged sword. W. W. Norton. [aJH]

List, J. A. (2004) Neoclassical theory versus Prospect theory: Evidence from the marketplace. Econometrica 72:615-25. [aJH]

Liu, D., Wellman, H. M., Tardif, T. \& Sabbagh, M. A. (2008) Theory of mind development in Chinese children: A meta-analysis of false-belief understanding across cultures and languages. Developmental Psychology 44(2):523-31. $[\mathrm{aJH}]$
Long, J. C. \& Kittles, R. A. (2003) Human genetic diversity and nonexistence of biological races. Human Biology 75:449-71. [AMar]

Low, B. S. (2000) Why sex matters: A Darwinian look at human behavior. Princeton University Press. [aJH]

Luria, A. R. (1976) Cognitive development: Its cultural and social foundations. Harvard University Press. [aJH, DFL]

Lutz, C. (1988) Unnatural emotions: Everyday sentiments on a Micronesian atoll and their challenge to Western theory. University of Chicago Press. [DMTF]

Lyn, H., Russell, J. L. \& Hopkins, W. D. (2010) The impact of environment on the comprehension of declarative communication in apes. Psychological Science 21:360-65. [DAL]

MacDonald, S., Nyberg, L. \& Backman, L. (2006) Intra-individual variability in behavior: Links to brain structure, neurotransmission and neuronal activity. Trends in Neurosciences 29:474-80. [SSK]

Machery, E. (forthcoming) Variation in intuitions about reference and ontological disagreements. In: A companion to relativism, ed. S. D. Hales. Blackwell. [EM]

Machery, E., Mallon, R., Nichols, S. \& Stich, S. (2004) Semantics, cross-cultural style. Cognition 92:B1-12. [EM, SS]

Machery, E., Olivola, C. Y. \& de Blanc, M. (2009) Linguistic and metalinguistic intuitions in the philosophy of language. Analysis 69:689-94. [EM, SS]

Machery, E. \& Stich, S. P. (forthcoming) Experimental philosophy of language. In: The Routledge companion to philosophy of language, ed. D. G. Fara \& G. Russell. Routledge. [EM]

Madsen, M. C. (1971) Developmental and cross-cultural differences in the cooperative and competitive behavior of young children. Journal of Cross-Cultural Psychology 2(4):365-71. [DFL]

Majid, A. (2010) Words for parts of the body. In Words and the mind: How words capture human experience, ed. B. C. Malt \& P. Wolff, pp. 58-71. Oxford University Press. [AMaj]

Majid, A., Bowerman, M., Kita, S., Haun, D. B. M. \& Levinson, S. C. (2004) Can language restructure cognition? The case for space. Trends in Cognitive Sciences 8(3):108-14. [aJH]

Mallon, R., Machery, E., Nichols, S. \& Stich, S. (2009) Against arguments from reference. Philosophy and Phenomenological Research 79(2):332-56. [SS]

Mann, V. A., Sasanuma, S., Sakuma, N. \& Masaki, S. (1990) Sex differences in cognitive abilities: A cross-cultural perspective. Neuropsychologia 28:106377. [aJH]

Markus, H. R. \& Kitayama, S. (1991) Culture and the self: Implications for cognition, emotion, and motivation. Psychological Review 98:224-53. [aJH, SK]

Marlowe, F. (2004) Mate preferences among Hadza hunter-gatherers. Human Nature 15(4):365-76. [aJH]

Marlowe, F., Apicella, C. \& Reed, D. (2005) Men's preferences for women's profile waist-to-hip ratio in two societies. Evolution and Human Behavior 26(6):45868. [aJH]

Marlowe, F. \& Wetsman, A. (2001) Preferred waist-to-hip ratio and ecology. Personality and Individual Differences 30(3):481-89. [aJH]

Maryanski, A. \& Turner, J. (1992) The social cage: Human nature and the origins of society. Stanford University Press. [AMar]

Masuda, T. \& Nisbett, R. E. (2001) Attending holistically versus analytically: Comparing the context sensitivity of Japanese and Americans. Journal of Personality and Social Psychology 81:992-34. [aJH]

May, R. M. (1997) The scientific wealth of nations. Science 275:793-96. [arJH]

McCauley, R. \& Henrich, J. (2006) Susceptibility to the Müller-Lyer illusion, theory-neutral observation, and the diachronic penetrability of the visual input system. Philosophical Psychology 19(1):1-23. [aJH]

McCrae, R. R., Terraciano, A. \& 79 members of the Personality Profiles of Culture Project (2005) Universal features of personality traits from the observer's perspective: Data from 50 cultures. Journal of Personality and Social Psychology 88:547-61. [aJH]

McDonough, L., Choi, S. \& Mandler, J. M. (2003) Understanding spatial relations: Flexible infants, lexical adults. Cognitive Psychology 46:229-59. [AMaj]

Mead, M. (1932) An investigation of the thought of primitive children, with special reference to animism. The Journal of the Royal Anthropological Institute of Great Britain and Ireland 62:173-90. [rJH, RAS]

Medin, D. L. \& Atran, S. (2004) The native mind: Biological categorization and reasoning in development and across cultures. Psychological Review $111(4): 960-83 . \quad[\mathrm{aJH}]$

Medin, D. L. \& Bang, M. (2008) Perspective taking, diversity and partnerships. American Psychological Association 22(2). (APA Science Directorate Online publication, February 2008.) Available at: http://www.apa.org/science/about/ psa/2008/02/medin.aspx [WMB, aJH]

Medin, D. L., Bennis, W. M. \& Chandler, M. (under review) Culture and the homefield disadvantage. Perspectives on Psychological Science. [WMB]

Mei, J. (1994) The Northern Chinese custom of rearing babies in sandbags: Implications for motor and intellectual development. In: Motor development: Aspects of normal and delayed development, ed. J. van Rossum \& J. Laszlo, pp. 41-48. VU Uitgeverij. [LBK] 
Mesquita, B. \& Frijda, N. H. (1992) Cultural variations in emotions: A review. Psychological Bulletin 112(2):179-204. [DMTF]

Mezulis, A. H., Abramson, L. Y., Hyde, J. S. \& Hankin, B. L. (2004) Is there a universal positive bias in attributions? A meta-analytic review of individual, developmental, and cultural differences in the self-serving attributional bias. Psychological Bulletin 130:711-47. [arJH]

Miller, J. G. (1984) Culture and the development of everyday social explanation. Journal of Personality and Social Psychology 46:961-78. [aJH]

Miller, J. G. \& Bersoff, D. M. (1992) Culture and moral judgment: How are conflicts between justice and interpersonal responsibilities resolved? Journal of Personality and Social Psychology 62:541-54. [aJH]

Miller, J. G., Bersoff, D. M. \& Harwood, R. L. (1990) Perceptions of social responsibilities in India and the United States: Moral imperatives or personal decisions? Journal of Personality and Social Psychology 58:33-47. [aJH]

Milner, E. (1951) A study of the relationship between reading readiness in gradeone children and patterns of parent-child interaction. Child Development 22:95-112. [AF $]$

Mitani, J. \& Watts, D. (2001) Why do chimpanzees hunt and share meat? Animal Behaviour 61:1-10. [CB]

Mitani, J. \& Watts, D. (2005) Correlates of territorial boundary patrol behaviour in wild chimpanzees. Animal Behaviour 70:1079-86. [CB]

Mitani, J., Watts, D. \& Muller, M. (2002) Recent development in the study of wild chimpanzee behaviour. Evolutionary Anthropology 11(1):9-25. [CB]

Miyamoto, Y. \& Kitayama, S. (2002) Cultural variation in correspondence bias: The critical role of attitude diagnosticity of socially constrained behavior. Journal of Personality and Social Psychology 83:1239-48. [aJH]

Moghaddam, F. M. (1987) Psychology in three words. American Psychologist 42:912-20. [LBK]

Mook, D. G. (1983) In defense of external invalidity. American Psychologist 38(4):379-87. [aJH, VJK]

Moore, F. R., Cassidy, C., Law Smith, M. J. \& Perrett, D. I. (2006) The effects of female control of resources on sex-differentiated mate preferences. Evolution and human behavior 27(3):193-205. [aJH]

Morling, B. \& Lamoreaux, M. (2008) Measuring culture outside the head: A metaanalysis of individualism-collectivism in cultural products. Personality and Social Psychology Review 12:199-21. [aJH]

Morris, M. \& Peng, K. (1994) Culture and cause: American and Chinese attributions for social and physical events. Journal of Personality and Social Psychology 67:949-71. [aJH]

Na, J., Grossmann, I., Varnum, M.E.W., Gonzalez, R., Kitayama, S., \& Nisbett, R.E. (in press) When cultural differences are not reducible to individual differences. Proceedings of the National Academy of Science. [aJH]

Neisser, U. (1963) The multiplicity of thought. British Journal of Psychology 54:114. $[\mathrm{aJH}]$

Nelson, C., Zeanah, C., Fox, N., Marshall, P., Smyke, A. \& Guthrie, D. (2007) Cognitive recovery in socially deprived young children: The Bucharest early intervention project. Science 318:1937-40. [CB]

Ng, S. H. \& Lai, J. C. L. (2009) Effects of culture priming on the social connectedness of the bicultural self. Journal of Cross-Cultural Psychology 40:170 86. $[\mathrm{SSK}]$

Ngui, P. W. (1969) The koro epidemic in Singapore. Australian and New Zealand Journal of Psychiatry 3:263-66. [rJH]

Nichols, S., Stich, S. \& Weinberg, J. (2003) Meta-skepticism: Meditations on ethnoepistemology. In: The skeptics, ed. S. Luper, pp. 227-47. Ashgate. [SS]

Nielsen, M. (2008) The future of science. Available at: http://michaelnielsen.org/ blog/the-future-of-science-2/. [MM]

Nisbett, R. E. (2003) The geography of thought: How Asians and Westerners think differently ... and why. Free Press. [arJH]

Nisbett, R. E. (2009) Intelligence and how to get it: Why schools and cultures count. Norton. [aJH]

Nisbett, R. \& Cohen, D. (1996) Culture of honor: The psychology of violence in the South. Westview Press. [WMB, rJH]

Nisbett, R. E., Peng, K., Choi, I. \& Norenzayan, A. (2001) Culture and systems of thought: Holistic versus analytic cognition. Psychological Review 108:291310. [aJH]

Norenzayan, A. (2006) Evolution and transmitted culture. Psychological Inquiry $17(2): 123-28 . \quad[\mathrm{aJH}]$

Norenzayan, A., Choi, I. \& Nisbett, R. E. (2002a) Cultural similarities and differences in social inference: Evidence from behavioral predictions and lay theories of behavior. Personality and Social Psychology Bulletin 28(1):109-20. $[\mathrm{aJH}]$

Norenzayan, A., Choi, I. \& Peng, K. (2007) Cognition and perception. In: Handbook of cultural psychology, ed. S. K. D. Cohen, pp. 569-94. Guilford Press. $[\mathrm{a} \mathrm{J} \mathrm{H}]$

Norenzayan, A. \& Heine, S. J. (2005) Psychological universals: What are they and how can we know? Psychological Bulletin 131:763-84. [arJH]

Norenzayan, A., Henrich, J. \& McElreath, R. (n.d.) More Chinese than the Chinese. University of British Columbia. [aJH]
Norenzayan, A., Smith, E. E., Kim, B. J. \& Nisbett, R. E. (2002b) Cultural preferences for formal versus intuitive reasoning. Cognitive Science 26(5):653-84. [arjH]

Nowak, M. A., Page, K. M. \& Sigmund, K. (2000) Fairness versus reason in the Ultimatum Game. Science 289(5485):1773-75. [aJH]

O’Mara, E. M., Gaertner, L., Sedikides, C., Zhou, X. \& Liu, Y. (2009) On the panculturality of self-enhancement: Self-enhancement promotes psychological adjustment in the US and China. Unpublished manuscript, University of Tennessee. [LG]

Ochs, E. \& Schieffelin, B. B. (1984) Language acquisition and socialization: Three developmental stories and their implications. In: Culture theory: Essays on mind, self, and society, ed. R. Shweder \& R. L. LeVine, pp. 276-320. Cambridge University Press. [DFL]

Oishi, S. (in press) The psychology of residential mobility: Implications for the self, social relationships, and well-being. Perspectives on Psychological Science. [SK]

Oishi, S. \& Graham, J. (in press) Social ecology: Lost and found in psychological science. Perspectives on Psychological Science. [SK]

Oishi, S., Ishii, K. \& Lun, J. (2009a) Residential mobility and conditionality of group identification. Journal of Experimental Social Psychology 45:913-19. [SK]

Oishi, S., Kesebir, S. \& Snyder, B. H. (2009b) Sociology: A lost connection in social psychology. Personality and Social Psychology Review 13:334-53. [SK]

Oishi, S., Lun, J. \& Sherman, G. D. (2007) Residential mobility, self-concept, and positive affect in social interactions. Journal of Personality and Social Psychology 93:131-41. [SK]

Okazaki, S. (1997) Sources of ethnic differences between Asian American and White American college students on measures of depression and social anxiety. Journal of Abnormal Psychology 106:52-60. [r]H]

Oxoby, R. J. \& Spraggon, J. (2008) Mine and yours: Property rights in dictator games. Journal of Economic Behavior and Organization 65(3-4):703-13. [NB]

Oyserman, D., Coon, H. M. \& Kemmelmeier, M. (2002) Rethinking individualism and collectivism: Evaluation of theoretical assumptions and meta-analyses. Psychological Bulletin 128:1773-75. [aJH]

Oyserman, D. \& Lee, S. (2008) Does culture influence what and how we think? Effects of priming individualism and collectivism. Psychological Bulletin 134(2):311-42. [aJH]

Özgen, E. \& Davies, I. R. L. (1998) Turkish color terms: Tests of Berlin and Kay's theory of color universals and linguistic relativity. Linguistics 36:919-56. [AMaj]

Parke, R. D. (2000) Beyond white and middle class: Cultural variations in families assessments, processes, and policies. Journal of Family Psychology 14:33133. $[\mathrm{LBK}]$

Pasupathi, M. (1999) Age differences in response to conformity pressure for emotional and nonemotional material. Psychology and Aging 14:170-74. $[\mathrm{aJH}]$

Paulhus, D. L. (1998) Interpersonal vs. intrapsychic adaptiveness of trait selfenhancement: A mixed blessing? Journal of Personality and Social Psychology $74: 1197-1208 . \quad[\mathrm{rJH}]$

Peng, K. \& Nisbett, R. E. (1999) Culture, dialectics, and reasoning about contradiction. American Psychologist 54(9):741-54. [aJH]

Peng, K., Nisbett, R. E. \& Wong, N. Y. C. (1997) Validity problems comparing values across cultures and possible solutions. Psychological Methods 2:32944. [aJH]

Peterson, R. A. (2001) On the use of college students in social science research Insights from a second-order meta-analysis. Journal of Consumer Research 28:450-61. [aJH]

Piaget, J. (1929) The child's conception of the world. Harcourt, Brace and Company. [RAS]

Pica, P., Lerner, C., Izard, V. \& Dehaene, S. (2004) Exact and approximate arithmetic in an Amazonian indigenous group. Science 306:499-501. [arjH]

Pika, S. \& Mitani, J. (2006) Referential gestural communication in wild chimpanzees (Pan troglodytes). Current Biology 16:R191-92. [DAL]

Pillsworth, E. G. (2008) Mate preferences among the Shuar of Ecuador: Trait rankings and peer evaluations. Evolution and Human Behavior 29(4):25667. [aJH]

Pinker, S. (1994) The language instinct: How the mind creates language. Harper. $[\mathrm{AF}]$

Plato (1892) The Republic. In: The dialogues of Plato, vol. I, trans. B. Jowett. Random House. [SS]

Pouliasi, K. \& Verkuyten, M. (2007) Networks of meaning and the bicultural mind: A structural equation modeling approach. Journal of Experimental Social Psychology 43:955-63. [SSK]

Povinelli, D. J. (2000) Folk physics for apes: The chimpanzee's theory of how the world works. Oxford University Press. [CB]

Povinelli, D. J., Bering, J. M. \& Giambrone, S. (2003) Chimpanzee "pointing”: Another error of the argument by analogy? In: Pointing: Where language culture, and cognition meet, ed. S. Kita, pp. 35-68. Erlbaum. [DAL] 
Povinelli, D. J. \& Davis, D. R. (1994) Differences between chimpanzees (Pan troglodytes) and humans (Homo sapiens) in the resting state of the index finger: Implications for pointing. Journal of Comparative Psychology 108:134-39. [DAL]

Povinelli, D. J. \& Vonk, J. (2003) Chimpanzee minds: Suspiciously human? Trends in Cognitive Sciences 7(4):157-60. [CB]

Price-Williams, D., Gordon, W. \& Ramirez, M. (1969) Skill and conservation: A study of pottery-making children. Developmental Psychology 1(6):769. [DFL]

Putnam, R. (2000) Bowling alone: The collapse and revival of American community. Simon and Schuster. [aJH]

Racine, T. P., Leavens, D. A., Susswein, N. \& Wereha, T. J. (2008) Pointing as intersubjectivity in human and nonhuman primates. In: Enacting intersubjectivity: A cognitive and social perspective to the study of interactions, ed. F. Morganti, A. Carassa \& G. Riva, pp. 65-79. IOS Press. [DAL]

Ramey, C. T. \& Ramey, S. L. (2004) Early learning and school readiness: Can early intervention make a difference? Merrill-Palmer Quarterly 50:471-91. [AF

Regier, T., Kay, P. \& Cook, R. S. (2005) Focal colors are universal after all. Proceedings of the National Academy of Sciences USA 102(23):8386-91. [aJH]

Reifman, A., Klein, J. G. \& Murphy, S. T. (1989) Self-monitoring and age. Psychology and Aging 4:245-46. [aJH]

Reips, U.-D. \& Krantz, J. H. (2010) Conducting true experiments on the Web. In: Advanced methods for behavioral research on the Internet, ed. S. D. Gosling \& J. A. Johnson, pp. 193-216. American Psychological Association. [SDG]

Reis, H. T. \& Gosling, S. D. (2010) Social psychological methods outside the laboratory. In: Handbook of social psychology, vol. 1, 5th edition, ed. S. T. Fiske, D. T. Gilbert \& G. Lindzey, pp. 82-114. Wiley. [SDG]

Relethford, J. H. (2002) Apportionment of global human genetic diversity bias on craniometrics and skin color. American Journal of Physical Anthropology 118:393-98. [AMar]

Rennie, L. J. \& Dunne, M. (1994) Gender, ethnicity, and student's perceptions about science and science-related careers in Fiji. Science Education 78:285-300. [aJH]

Reynolds Losin, E. A., Dapretto, M. \& Iacoboni, M. (2010) Culture and neuroscience: Additive or synergestic. Social Cognitive and Affective Neuroscience. DOI: $10.1093 /$ scan $/ \mathrm{nsp} 058 \quad$ [rJH]

Richerson, P. J. \& Boyd, R. (2005) Not by genes alone: How culture transformed human evolution. University of Chicago Press. [arJH, KP]

Richerson, P. J., Boyd, R. \& Bettinger, R. L. (2001) Was agriculture impossible during the Pleistocene but mandatory during the Holocene? A climate change hypothesis. American Antiquity 66:387-411. [KP]

Rivers, W. H. R. (1901a) Color vision. In: Reports of the Cambridge anthropological expedition to the Torres Straits, vol. 2: Physiology and psychology, ed. A. C. Haddon, pp. 48-96. Cambridge University Press. [aJH]

Rivers, W. H. R. (1901b) Introduction and vision. In: Reports of the Cambridge anthropological expedition to the Torres Straits, ed. A. C. Haddon. Cambridge University Press. [rJH]

Roberson, D., Davies, I. \& Davidoff, J. (2000) Color categories are not universal: Replications and new evidence from a stone-age culture. Journal of Experimental Psychology: General 129(3):369-98. [aJH]

Roberson, D., Pak, H. S. \& Hanley, J. R. (2008) Categorical perception of colour in the left and right hemisphere is verbally mediated: Evidence from Korean. Cognition 107:752-62. [AMaj]

Robins, R. W. \& Beer, J. S. (2001) Positive illusions about the self: Short-term benefits and long-term costs. Journal of Personality and Social Psychology 80:340-52. [rJH

Robson, P. (1984) Prewalking locomotor movements and their use in predicting standing and walking. Child: Care, health, and development 10:317-30. [LBK]

Rogers, C. R. (1951) Client-centered therapy. Houghton Mifflin. [aJH]

Rogoff, B. (2003) The cultural nature of human development. Oxford University Press. [aJH]

Ross, L. D., Amabile, T. M. \& Steinmetz, J. L. (1977) Social roles, social control, and biases in social-perception processes. Journal of Personality and Social Psychology 35:485-94. [aJH]

Ross, M., Heine, S. J., Wilson, A. E. \& Sugimori, S. (2005) Cross-cultural discrepancies in self-appraisals. Personality and Social Psychology Bulletin 31:1175-88. [rJH]

Ross, N., Medin, D. L., Coley, J. D. \& Atran, S. (2003) Cultural and experiential differences in the development of folkbiological induction. Cognitive Development 18(1):25-47. [aJH]

Rozin, P. (2001) Social psychology and science: Some lessons from Solomon Asch. Personality and Social Psychology Review 5:2-14. [aJH]

Rozin, P. (2003) Five potential principles for understanding cultural differences in relation to individual differences. Journal of Research in Personality 37(4):273-83. [aJH, PRoz]

Rozin, P. (2009) What kind of empirical research should we publish, fund, and reward? A different perspective. Perspectives on Psychological Science 4:43539. [aJH, PRoc]
Rozin, P., Fischler, C., Shields, C. \& Masson, E. (2006) Attitudes towards large numbers of choices in the food domain: A cross-cultural study of five countries in Europe and the USA. Appetite 46:304-308. [aJH]

Rozin, P. \& Nemeroff, C. J. (1990) The laws of sympathetic magic: A psychological analysis of similarity and contagion. In: Cultural psychology: Essays on comparative human development, ed. J. Stigler, G. Herdt \& R. A. Shweder, pp. 205-32. Cambridge University Press. [arJH]

Russell, R. (1984) Psychology in its world context. American Psychologist 39:101725. $[\mathrm{LBK}]$

Sakai, M., Ishikawa, S., Takizawa, M., Sato, H. \& Sakano, Y. (2004) The state of Hikikomori from a family's point of view: Statistical survey and the role of psychological intervention. Japanese Journal of Counseling Science 37:16879. $[\mathrm{rJH}]$

Sanchez-Burks, J. (2005) Protestant relational ideology: The cognitive underpinnings and organizational implications of an American anomaly. Research in Organizational Behavior: An Annual Series of Analytical Essays and Critical Reviews 26:265-305. [aJH]

Sanz, C., Morgan, D. \& Gulick, S. (2004) New insights into chimpanzees, tools, and termites from the Congo Basin. American Naturalist 164(5):567-81. [CB]

Saucier, G., Georgiades, S., Tsaousis, I. \& Goldberg, L. R. (2005) The factor structure of Greek personality adjectives. Journal of Personality and Social Psychology 88:856-75. [aJH]

Savage-Rumbaugh, E. S. (1986) Ape language: From conditioned response to symbol. Columbia University Press. [DAL]

Savani, K., Markus, H. R. \& Conner, A. L. (2008) Let your preference be your guide? Preferences and choices are more tightly linked for North Americans than for Indians. Journal of Personality and Social Psychology 95:861-76. $[\mathrm{aJH}]$

Savani, K., Markus, H. R., Naidu, N. V. R., Kumar, S. \& Berlia, N. (in press) What counts as choice? Americans are more likely than Indians to construe actions as choices. Psychological Science. [aJH]

Scarlett, W. G., Naudeau, S., Salonius-Pasternak, D. \& Ponte, I. (2005) Children's play. Sage. [DFL]

Schaller, M. \& Murray, D. R. (2008) Pathogens, personality, and culture: Disease prevalence predicts worldwide variability in sociosexuality, extraversion, and openness to experience. Journal of Personality and Social Psychology 95(1):212-21. [aJH]

Schlenker, H. R. (1974) Social psychology and science. Journal of Personality and Social Psychology 29:1-15. [LG]

Schmitt, D. P. (2005) Sociosexuality from Argentina to Zimbabwe: A 48-nation study of sex, culture, and strategies of human mating. Behavioral and Brain Sciences 28(2):247-311. [aJH]

Schmitt, D. P., Allik, J., McCrae, R. R., Benet-Martinez, V., Alcaly, L., Ault, L., Austers, I., Bennett, K. L., Bianchi, G., Boholst, F., Cunen, M., Braeckman, J., Brainerd, E. G., Gerard, L., Caron, G., Casullo, M. M., Cunningham, M. Daibo, I., de Backer, C., Desouza, E., Diaz-Loving, R., Diniz, G., Durkin, K., Echegaray, M., Eremsoy, E., Euler, H. A., Falzon, R., Fisher, M. L., Foley, D., Fry, D. P., Fry, S., Ghayar, M. A., Giri, V. N., Golden, D. L., Grammer, K., Grimaldi, L., Halberstadt, L., Hague, S., Herrera, D., Hertel, J., Hoffmann, H., Hooper, D., Hradilekova, Z., Jaafar, J., Jankauskaite, M., Kabanagu-Stahel, H., Kardum, I., Khoury, N., Kwon, H., Laidra, K., Laireiter, A. R., Lakerveld, D., Lampert, A., Lauri, M., Lavallee, M., Lee, S. J., Leung, L. C., Locke, K. D., Locke, V., Luksik, I., Magaisa, I., Marcinkeviciene, D., Mata, A., Mata, R., McCarthy, B., Mills, M. E., Mikhize, N. J., Moreira, J., Moreira, S., Moya, M. Munyae, M., Noller, P., Olimar, H., Opre, A., Panaviotou, A., Petrovic, N., Poels, K., Popper, M., Poulimenou, M., P’yatokh, V., Raymond, M., Reips, U. D., Reneau, S. E., Rivera-Aragon, S., Rowatt, W. C., Ruch, W., Rus, V. S., Safir, M. P., Salas, S., Sambataro, F., Sandnabba, K. N., Schulmeyer, M. K. Schutz, A., Scrimali, T., Shackelford, T. K., Sharan, M. B., Shaver, P. R., Sichona, F., Simonetti, F., Sineshaw, T., Sookdew, R., Spelman, T., Spyron, S., Sumer, H. C., Sumer, N., Supekova, M., Szlendak, T., Taylor, R., Timmermans, B., Tooke, W., Tsaousis, I., Tungaranza, F. S. K., Van Overwalle, F., Vandermassen, G., Vanhoomissen, T., Vanwesenbeeck, I., Vasey, P. L., Verissimo, J., Voracek, M., Wan, W. W. N., Wang, T. W., Weiss, P., Wijaya, A Woertman, L., Youn, G. \& Zupaneic, A. (2007) The geographic distribution of big five personality traits: Patterns and profiles of human self-description across 56 nations. Journal of Cross-Cultural Psychology 38:173-212. [aJH]

Schroeders, U., Wilhelm, O. \& Schipolowski, S. (2010) Internet-based ability testing. In: Advanced methods for behavioral research on the Internet, ed. S. D. Gosling \& J. A. Johnson, pp. 131-48. American Psychological Association. [SDG]

Schwartz, B. (2004) The paradox of choice: Why more is less. Harper-Collins. [aJH]

Schwartz, S. H. \& Bilsky, W. (1990) Toward a theory of the universal content and structure of values: Extensions and cross-cultural replications. Journal of Personality and Social Psychology 58:878-91. [aJH]

Searle, J. (1980) Minds, brains and programs. Behavioral and Brain Sciences $3(3): 417-57 . \quad[\mathrm{SS}]$ 
Sears, D. (1986) College sophomores in the laboratory: Influences of a narrow data base on social psychology's view of human nature. Journal of Personality and Social Psychology 51:515-30. [aJH]

Sedikides, C., Gaertner, L. \& Toguchi, Y. (2003) Pancultural self-enhancement. Journal of Personality and Social Psychology 84:60-79. [LG, rJH]

Sedikides, C., Gaertner, L. \& Vevea, J. L. (2005) Pancultural self-enhancement reloaded: A meta-analytic reply to Heine (2005). Journal of Personality and Social Psychology 89:539-51. [LG, rJH]

Sedikides, C., Gaertner, L. \& Vevea, J. L. (2007a) Evaluating the evidence for pancultural self-enhancement. Asian Journal of Social Psychology 10:20103. [LG, rJH]

Sedikides, C., Gaertner, L. \& Vevea, J. L. (2007b) Inclusion of theory-relevant moderators yield the same conclusions as Sedikides, Gaertner, and Vevea (2005) A meta-analytical reply to Heine, Kitayama, and Hamamura (2007). Asian Journal of Social Psychology 10:59-67. [LG, rJH]

Sedikides, C. \& Gregg, A. P. (2008) Self-enhancement: Food for thought. Perspectives on Psychological Science 3:102-16. [LG]

Segall, M. H., Campbell, D. T. \& Herskovits, M. J. (1966) The influence of culture on visual perception. Bobbs-Merrill. [WMB, arJH, LBK]

Sexton, V. S. \& Misiak, H. (1984) American psychologist and psychology abroad. American Psychologist 39:1026-31. [LBK]

Shariff, A. F. \& Norenzayan, A. (2007) God is watching you - Priming god concepts increases prosocial behavior in an anonymous economic game. Psychological Science 18(9):803-809. [aJH]

Sheth, J. N. (1970) Are there differences in dissonance reduction behavior among students and housewives? Journal of Marketing Research 7:243-45. [aJH]

Shweder, R. (1990) Cultural psychology: What is it? In: Cultural psychology: Essays on comparative human development, ed. J. Stigler, R. A. Shweder \& G. Herdt, pp. 1-46. Cambridge University Press. [RAS]

Shweder, R. (1991) Thinking through cultures: Expeditions in cultural psychology. Harvard University Press. [RAS]

Shweder, R. (2003) Why do men barbecue?: Recipes for cultural psychology. Harvard University Press. [RAS]

Shweder, R. A. \& Bourne, E. J. (1982) Does the concept of the person vary crossculturally? In: Cultural conceptions of mental health and therapy, ed. A. J. Marsella \& G. M. White, pp. 97-137. Kluwer. [aJH]

Shweder, R. A., Much, N. C., Mahapatra, M. \& Park, L. (1997) The "big three" of morality (autonomy, community, divinity) and the "big three" explanations of suffering. In: Morality and health, ed. A. M. Brandt \& P. Rozin, pp. 119-69. Routledge. [aJH]

Siegler, R. S. (1996) Emerging minds: The process of change in children's thinking. Oxford University Press. [SSK]

Silk, J. B., Brosnan, S. F., Vonk, J., Henrich, J., Povinelli, D. J., Richardson, A. S., Lambeth, S. P., Mascaro, J. \& Shapiro, S. J. (2005) Chimpanzees are indifferent to the welfare of unrelated group members. Nature 437:1357-59. $[\mathrm{CB}, \mathrm{rJH}]$

Sillitoe, P. (1998) An introduction to the anthropology of Melanesia: Culture and tradition. Cambridge University Press. $[\mathrm{r} J \mathrm{H}]$

Singh, D. (2006) Universal allure of the hourglass figure: An evolutionary theory of female physical attractiveness. Clinics in Plastic Surgery 33(3):359-70. [aJH]

Singh, D. \& Luis, S. (1994) Ethnic and gender consensus for the effect of WHR on judgment of women's attractiveness. Human Nature 6:51-65. [aJH]

Slingerland, E. G. (2008) What science offers the humanities: Integrating body and culture. Cambridge University Press. [arJH]

Slobin. D. I. (1973) Cognitive prerequisites for the development of grammar. In: Studies of child language development, ed. C. A. Ferguson \& D. I. Slobin, pp. 175-208. Holt, Rinehart \& Winston. [AMaj]

Sloman, S. (1996) The empirical case for two systems of reasoning. Psychological Bulletin 119:22-30. [aJH]

Smith, T., Tafforeau, P., Reid, D., Grun, R., Eggins, S., Boutakiout, M. \& Hublin, J. (2007) Earliest evidence of modern human life history in North Africa early Homo sapiens. Proceedings of the National Academy of Sciences of the United States of America 104:6128-33. [AMar]

Smith, V. L. (2010) Theory and experiment: What are the questions? Journal of Economic Behavior and Organization 73(1):3-15. [SG]

Snarey, J. R. (1985) The cross-cultural universality of social-moral development: A critical review of Kohlbergian research. Psychological Bulletin 97(2):20232. $[\mathrm{aJH}]$

Snibbe, A. C. \& Markus, H. R. (2005) You can't always get what you want: Social class, agency, and choice. Journal of Personality and Social Psychology $88: 703-20$. [aJH]

Sosa, E. (2009) The use of intuitions in philosophy. In: Stich and his critics, ed. D. Murphy \& M. Bishop, pp. 101-112. Wiley-Blackwell. [SS]

Sousa, P., Atran, S. \& Medin, D. L. (2002) Essentialism and folkbiology: Evidence from Brazil. Journal of Cognition and Culture 2:195-223. [aJH]

Spelke, E. S. \& Kinzler, K. D. (2007) Core knowledge. Developmental Science 10:89-96. [AF]
Sperber, D., Cara, F. \& Girotto, V. (1995) Relevance theory explains the selection task. Cognition 57(1):31-95. [NB]

Srivastava, S., John, O. P., Gosling, S. D. \& Potter, J. (2003) Development of personality in early and middle adulthood: Set like plaster or persistent change? Journal of Personality and Social Psychology 84:1041-53. [SDG]

Starmans, C. \& Friedman, O. (2009) Is knowledge subjective? A sex difference in adults' epistemic intuitions. Poster presented at the Biennial Meeting of the Cognitive Development Society, October 16-17, 2009, San Antonio, TX. [SS]

Stephens, N. M., Markus, H. R. \& Townsend, S. S. M. (2007) Choice as an act of meaning: The case of social class. Journal of Personality and Social Psychology 93:814-30. [aJH]

Stich, S. (2009) Reply to Sosa. In: Stich and his critics, ed. D. Murphy \& M. Bishop, pp. 228-36. Wiley-Blackwell. [SS]

Stipek, D. (1995) The development of pride and shame in toddlers. In: Selfconscious emotions: The psychology of shame, guilt, embarrassment, and pride, ed. J. P. Tangney \& K. W. Fischer, pp. 237-52. Guilford Press. [DFL]

Strauss, S. \& Ziv, M. (2004) Teaching: Ontogenesis, culture, and education. Cognitive Development 19:451-56. [DFL]

Streeter, S. A. \& McBurney, D. H. (2003) Waist-hip ratio and attractiveness: New evidence and a critique of "a critical test." Evolution and Human Behavior 24(2):88-98. [aJH]

Stutchbury, B. J. M. \& Morton, E. S. (2001) Behavioral ecology of tropical birds. Academic Press. [SK]

Su, J. C. \& Oishi, S. (2010) Culture and self-enhancement. A social relation analysis. Unpublished manuscript, University of Virginia. [rJH]

Sue, S. (1999) Science, ethnicity, and bias. Where have we gone wrong? American Psychologist 54:1070-77. [aJH]

Sugiyama, L. S. (2004) Is beauty in the context-sensitive adaptations of the beholder? Shiwiar use of waist-to-hip ratio in assessments of female mate value. Evolution and Human Behavior 25(1):51-62. [aJH]

Sugiyama, L. S., Tooby, J. \& Cosmides, L. (2002) Cross-cultural evidence of cognitive adaptations for social exchange among the Shiwiar of Ecuadorian Amazonia. Proceedings of the National Academy of Sciences USA 99(17):11537-42. [aJH]

Suh, E. M. (2002) Culture, identity consistency, and subjective well-being. Journal of Personality and Social Psychology 83:1378-19. [aJH]

Sung, J. \& Hsu, H. C. (2009) Korean mother's attention regulation and referential speech: Associations with language and play in 1-year-olds. International Journal of Behavioral Development 33(5):430-39. [DFL]

Super, C. M. (1976) Environmental effects on motor development: The case of "African infant precocity." Developmental Medicine and Child Neurology 18:561-67. [LBK]

Sutter, M. \& Kocher, M. (2007a) Age and the development of trust and reciprocity. Games and Economic Behavior 59:364-82. [aJH]

Sutter, M. \& Kocher, M. (2007b) Trust and trustworthiness across different age groups. Games and Economic Behavior 59(2):364-82. [SG]

Swami, V., Neto, F., Tovée, M. J. \& Furnham, A. (2007) Preferences for female body weight and shape in three European countries. European Psychologist $12(3): 220-28 . \quad[\mathrm{aJH}]$

Swami, V. \& Tovée, M. J. (2007) The relative contribution of profile body shape and weight to judgements of women's physical attractiveness in Britain and Malaysia. Body Image 4(4):391-96. [aJH]

Sweeney, P. D., Anderson, K. \& Bailey, S. (1986) Attributional style in depression: A meta-analytic review. Journal of Personality and Social Psychology 50:97491. [aJH]

Tanaka, T., Camerer, C. \& Nguyen, Q. (forthcoming) Risk and time preferences: Experimental and household survey data from Vietnam. American Economic Review. [aJH]

Tangney, J. P. \& Dearing, R. L. (2002) Shame and guilt. Guilford Press. [DMTF]

Taylor, S. E. \& Brown, J. D. (1988) Illusion and well-being: A social psychological perspective on mental health. Psychological Bulletin 103:193-210. [LG]

Taylor, S. E., Lerner, J. S., Sherman, D. K., Sage, R. M. \& McDowell, N. K. (2003) Portrait of the self-enhancer: Well-adjusted and well liked or maladjusted and friendless? Journal of Personality and Social Psychology $84: 165-76 . \quad[\mathrm{rJH}]$

Tennie, C., Gilby, I. \& Mundry, R. (2009) The meat-scrap hypothesis: Small quantities of meat may promote cooperative hunting in wild chimpanzees (Pan troglodytes). Behavioral Ecology and Sociobiology 63(3):421-31. [ [rJH]

Terrill, A. (2006) Body part terms in Lavukaleve, a Papuan language of the Solomon Islands. Language Sciences 28:304-22. [AMaj]

Tesser, A. (1988) Toward a self-evaluation maintenance model of social behavior. In: Advances in experimental social psychology, vol. 21, ed. L. Berkowitz, pp. 181-227. Academic Press. [aJH]

Thierry, G., Athanasopoulos, P., Wiggett, A., Dering, B. \& Kuipers, J.-R. (2009) Unconscious effects of language-specific terminology on preattentive color perception. Proceedings of the National Academy of Sciences USA 106:456770. [AMaj] 
Thomson, J. (1976) Killing, letting die, and the trolley problem. The Monist 59:20417. $[\mathrm{SS}]$

Titchener, E. B. (1916) On ethnological tests of sensation and perception with special reference to tests of color vision and tactile discrimination described in the reports of the Cambridge anthropological expedition to Torres Straits. Proceedings of the American Philosophical Society 55:204-36. [RAS]

Tomasello, M. (2006) Why don't apes point? In: Roots of human sociality: Culture, cognition and interaction, ed. N. Enfield \& S. C. Levinson, pp. 506-24. Berg. [DAL $]$

Tomasello, M., Carpenter, M., Call, J., Behne, T. \& Moll, H. (2005) Understanding and sharing intentions: The origins of cultural cognition. Behavioral and Brain Sciences 28:675-91. [CB]

Tooby, J. \& Cosmides, L. (1992) The psychological foundations of culture. In: The adapted mind: Evolutionary psychology and the generation of culture, ed. J. Barkow, L. Cosmides \& J. Tooby, pp. 19-136. Oxford University Press. [aJH]

Tracer, D. P. (2003) Selfishness and fairness in economic and evolutionary perspective: An experimental economic study in Papua New Guinea. Current Anthropology 44(3):432-38. [rJH]

Tracer, D. P. (2004) Market integration, reciprocity, and fairness in rural Papua New Guinea: Results from two-village Ultimatum Game experiments. In: Foundations of human sociality: Economic experiments and ethnographic evidence from fifteen small-scale societies, ed. J. Henrich, R. Boyd, S. Bowles, C. Camerer, E. Fehr \& H. Gintis, pp. 232-60. Oxford University Press. [rJH

Tracer, D. P., Mueller, I. \& Morse, J. (under review) Cruel to be kind: Effects of sanctions and third-party enforcers on generosity in Papua New Guinea. In: Experimenting with social norms: Fairness and punishment in cross-cultural perspective, ed. J. Henrich \& J. Ensminger. [rJH]

Tracy, J. L. \& Matsumoto, D. (2008) The spontaneous expression of pride and shame: Evidence for biologically innate nonverbal displays. Proceedings of the National Academy of Sciences USA 105(33):11655-60. [aJH]

Tracy, J. L. \& Robins, R. W. (2008) The nonverbal expression of pride: Evidence for cross-cultural recognition. Journal of Personality and Social Psychology 94(3):516-30. [DMTF, aJH]

Trettien, A. W. (1900) Creeping and walking. The American Journal of Psychology 12:1-57. [LBK]

Trevarthen, C. (1983) Interpersonal abilities of infants as generators for transmission of language and culture. In: The behavior of human infants, ed. A. Oliverio, pp. 145-76. Plenum Press. [DFL]

Triandis, H. C. (1980) Handbook of cross-cultural psychology. Allyn \& Bacon. [LBK]

Triandis, H. C. (1989) The self and social-behavior in differing cultural contexts. Psychological Review 96(3):506-20. [aJH]

Triandis, H. C. (1994) Culture and social behavior. McGraw-Hill. [aJH]

Triandis, H. C., McCusker, C. \& Hui, C. H. (1990) Multimethod probes of individualism and collectivism. Journal of Personality and Social Psychology 59:1006-20. [aJH]

Tropp, L. R. \& Wright, S. C. (2003) Evaluations and perceptions of self, ingroup, and outgroup: Comparisons between Mexican-American and EuropeanAmerican children. Self and Identity 2:203-21. [aJH]

Tsai, J. L. (2007) Ideal affect: Cultural causes and behavioral consequences. Perspectives on Psychological Science 19:242-59. [aJH]

Tseng, W.-S. (2001) Handbook of cultural psychiatry. Academic Press. [arjH]

Turkheimer, E., Haley, A., Waldron, M., D’Onofrio, B. \& Gottesman, I. I. (2003) Socioeconomic status modifies heritability of IQ in young children. Psychological Science 14(6):623-28. [aJH]

Turner, J. \& Maryanski, A. (2005) Incest: Origin of the taboo. Paradigm. [AMar]

Turner, J. \& Maryanski, A. (2008) On the origin of societies by natural selection. Paradigm. [AMar]

Tuten, T. L. (2010) Conducting online surveys. In: Advanced methods for behavioral research on the Internet, ed. S. D. Gosling \& J. A. Johnson, pp. 179-92. American Psychological Association. [SDG]

Tuttle, R., Webb, D. \& Baksh, M. (1991) Laetoli toes and Australopithecus afarensis. Human Evolution 6(3):193-200. [rJH]

Tuttle, R., Webb, D., Weidl, E. \& Baksh, M. (1990) Further progress on the Laetoli trails. Journal of Archaeological Science 17(3):347-62. [rJH]

Twenge, J. M. \& Campbell, W. K. (2001) Age and birth cohort differences in selfesteem: A cross-temporal meta-analysis. Personality and Social Psychology Review 5(4):321-44. [aJH]

Twenge, J. M. \& Im, C. (2007) Changes in the need for social approval, 1958-2001. Journal of Research in Personality 41:171-89. [aJH]

Uchikawa, K. \& Boynton, R. M. (1987) Categorical color perception of Japanese observers: Comparison with that of Americans. Vision Research 27:182533. [AMaj]

United Nations Development Programme (UNDP). (2006) Human development report, 2006. United Nations. [PRoc]

Uskul, A. K., Kitayama, S. \& Nisbett, R. E. (2008) Ecocultural basis of cognition: Farmers and fishermen are more holistic than herders.
Proceedings of the National Academy of Sciences USA 105(25):8552-56. $[\mathrm{aJH}, \mathrm{SK}]$

Van Boven, L., Kamada, A. \& Gilovich, T. (1999) The perceiver as perceived: Everyday intuitions about the correspondence bias. Journal of Personality and Social Psychology 77:1188-99. [aJH]

Varnum, M., Grossmann, I., Kitayama, S. \& Nisbett, R. E. (2008) The origin of cultural differences in cognition: The social orientation hypothesis. University of Michigan Press. [aJH]

Vazire, S. (2010) Online collection of informant reports. In: Advanced methods for behavioral research on the Internet, ed. S. D. Gosling \& J. A. Johnson, pp. 167-78. American Psychological Association. [SDG]

Vidal, F. (1994) Piaget before Piaget. Harvard University Press. [DFL]

Vohs, K. D., Baumeister, R. F., Schmeichel, B. J., Twenge, J. M., Nelson, N. M. \& Tice, D. M. (2008) Making choices impairs subsequent self-control: A limitedresource account of decision making, self-regulation, and active initiative. Journal of Personality and Social Psychology 94:883-98. [aJH]

Vonk, J., Brosnan, S., Silk, J., Henrich, J., Richardson, A., Lambeth, S., Schapiro, S. \& Povinelli, D. (2008) Chimpanzees do not take advantage of very low cost opportunities to deliver food to unrelated group members. Animal Behaviour $75(5): 1757-70 . \quad[\mathrm{CB}]$

Voyer, D., Voyer, S. \& Bryden, M. P. (1995) Magnitude of sex differences in spatial abilities: A meta-analysis and consideration of critical variables. Psychological Bulletin 117:250. [aJH]

Walker, R., Gurven, M., Hill, K., Migliano, A., Chagnon, N., De Souza, R., Djurovic, G., Hames, R., Hurtado, A. M., Kaplan, H., Kramer, K., Oliver, W. J., Valeggia, C. \& Yamauchi, T. (2006) Growth rates and life histories in twentytwo small-scale societies. American Journal of Human Biology 18(3):295311. $[\mathrm{rJH}]$

Wallace, B., Cesarini, D., Lichtenstein, P. \& Johannesson, M. (2007) Heritability of Ultimatum Game responder behavior. Proceedings of the National Academy of Sciences USA 104(40):15631-34. [aJH]

Wallbott, H. G. \& Scherer, K. R. (1986) How universal and specific is emotional experience? Evidence from 27 countries on five continents. Social Science Information 25(4):763-95. [DMTF]

Warneken, F. \& Tomasello, M. (2006) Altruistic helping in human infants and young chimpanzees. Science 311:1301-03. [CB]

Watters, E. (2010) Crazy like us: The globalization of the American psyche. Free Press. [rJH]

Waxman, S. \& Medin, D. L. (2007) Experience and cultural models matter: Placing firm limits on childhood anthropocentrism. Human Development 50(1):2330. [aJH]

Waxman, S., Medin, D. L. \& Ross, N. (2007) Folk-biological reasoning - from a cross-cultural developmental perspective: Early essentialist notions are shaped by cultural beliefs. Developmental Psychology 43(2):294-308. [aJH]

Webb, E. J., Campbell, D. T., Schwartz, R. D. \& Sechrest, L. (1966) Unobtrusive measures: Nonreactive research in the social sciences. Rand McNally. [VJK]

Weinberg, J., Nichols, S. \& Stich, S. (2001) Normativity and epistemic intuitions. Philosophical Topics 29(1\&2):429-60. [SS]

Weissman, M. M., Bland, R. C., Canino, G. J., Faravelli, C., Greenwald, S. \& Hwu, H.-G. (1996) Cross-national epidemiology of major depression and bipolar disorder. Journal of the American Medical Association 276:293-99. [rJH]

WEO Groups and Aggregates Information. (2009) IMF advanced economies list. (October 2009). Retrieved January 17, 2009, from World Economic Outlook, Database, available at: http://www.imf.org/external/pubs/ft/weo/2009/02/ weodata/groups.htm\#ae [SDG]

Whiten, A., Goodall, J., McGrew, W. C., Nishida, T., Reynolds, V., Sugiyama, Y., Tutin, C. E. G., Wrangham, R. W. \& Boesch, C. (1999) Cultures in chimpanzees. Nature 399(6737):682-85. [rJH]

Whittemore, R. D. (1989) Child caregiving and socialization to the Mandinka way: Toward an ethnography of childhood. Doctoral dissertation, University of California-Los Angeles. [DFL]

WHO (World Health Organization). (1973) The International Pilot Study of Schizophrenia. World Health Organization. $\quad[\mathrm{rJH}]$

Wicherts, J. M., Borsboom, D., Kats, J. \& Molenaar, D. (2006) The poor availability of psychological research for reanalysis. American Psychologist 61:726-28. [aJH]

Wight, V. R., Chau, M. \& Aratani, Y. (2010) Who are America's poor children? The official story. National Center for Children in Poverty: Columbia University. $[\mathrm{AF}]$

Wilkins, D. (2003) Why pointing with the index finger is not a universal (in sociocultural and semiotic terms). In: Pointing: Where language, culture, and cognition meet, ed. S. Kita, pp. 171-215. Erlbaum. [DAL]

Windschitl, P. D., Conybeare, D. \& Krizan, Z. (2008) Direct-comparison judgments: When and why above- and below-average effects reverse. Journal of Experimental Psychology: General 137:182-200. [rJH]

Wintre, M. G., North, C. \& Sugar, L. A. (2001) Psychologists' response to criticisms about research based on undergraduate participants: A developmental perspective. Canadian Psychology 42:216-25. [aJH] 
References/Henrich et al.: The weirdest people in the world?

Witkin, H. A. \& Berry, J. W. (1975) Psychological differentiation in cross-cultural perspective. Journal of Cross-Cultural Psychology 6(1):5-78. [aJH]

Witmer, L. (1909) A monkey with a mind. The Psychological Clinic 3:179-205. [DAL]

Wu, S. \& Keysar, B. (2007) Cultural effects on perspective taking. Psychological Science 18:600-606. [aJH]

Yamagishi, T., Hashimoto, H. \& Schug, J. (2008) Preferences versus strategies for culture-specific behavior. Psychological Science 19:579-84. [SK]

Yamaguchi, S., Greenwald, A. G., Banaji, M. R., Murakami, F., Chen, D., Shiomura, K., Kobayashi, C., Cai, H. \& Krendl, A. (2007) Apparent universality of positive implicit self-esteem. Psychological Science 18:498-500. [LG]

Yik, M. S. M., Russell, J. A., Ahn, C., Fernandez-Dols, J. M. \& Suzuki, N. (2002) Relating the five-factor model of personality to a circumplex model of affect: A five language study. In: The five-factor model of personality across cultures, ed. R. R. McCrae \& J. Allik, pp. 79-104. Kluwer. [aJH]

Yu, D. W. \& Shepard, G. H. (1998) Is beauty in the eye of the beholder? Nature 396(6709):321-22. [aJH]

Zebian, S. \& Denny, J. P. (2001) Integrative cognitive style in Middle

Eastern and Western groups. Journal of Cross-Cultural Psychology 32:5875. $[\mathrm{aJH}]$

Zelazo, P. R. (1983) The development of walking: New findings on old assumptions. Journal of Motor Behavior 2:99-137. [LBK]

Zhang, T. Y. \& Meaney, M. J. (2010) Epigenetics and the environmental regulation of the genome. Annual Review of Psychology 61:439-66. [AF]

Zhu, Y., Zhang, Li., Fan, J. \& Han. S. (2007) Neural basis of cultural influence on self representation. Neuroimage 34:1310-17. [JYC] 